\title{
Implementation and quantification of scanning transmission EBIC experiments for measuring nanometer diffusion lengths in manganite-titanite p-n heterojunctions
}

\author{
Dissertation \\ zur Erlangung des mathematisch-naturwissenschaftlichen \\ Doktorgrades \\ "Doctor rerum naturalium" \\ der Georg-August-Universität Göttingen \\ im Promotionsprogramm ProPhys \\ der Georg-August University School of Science (GAUSS) \\ vorgelegt von \\ Patrick Peretzki \\ aus Wabern
}

Göttingen, 2018 
Betreuungsauschuss:

Prof. Dr. Michael Seibt, IV. Physikalisches Institut, Georg-August-Universität Göttingen

Prof. Dr. Christian Jooß, Institut für Materialphysik, Georg-August-Universität Göttingen

Mitglieder der Prüfungskommission:

Referent: Prof. Dr. Michael Seibt, IV. Physikalisches Institut, Georg-August-Universität Göttingen

Korreferent: Prof. Dr. Christian Jooß, Institut für Materialphysik, Georg-AugustUniversität Göttingen

Weitere Mitglieder der Prüfungskommission:

Prof. Dr. Hans C. Hofsäss, II. Physikalisches Institut, Georg-August-Universität Göttingen

Prof. Dr. Wolfram Kollatschny, Institut für Astrophysik, Georg-August-Universität Göttingen

Prof. Dr. Vasily Moshnyaga, I. Physikalisches Institut, Georg-August-Universität Göttingen

Prof. Dr. Cynthia A. Volkert, Institut für Materialphysik, Georg-August-Universität Göttingen

Tag der mündlichen Prüfung:

19.12 .2018 


\section{Contents}

$\begin{array}{lll}1 & \text { Introduction } & 1\end{array}$

2 Theory 5

2.1 Photovoltaics in a $\mathrm{p}-\mathrm{n}$ junction $\ldots \ldots \ldots \ldots \ldots$

2.1 .1 Excess carriers in semiconductors . . . . . . . . . . . . . 5

2.1 .2 Generation, recombination and diffusion length . . . . . . . . . 7

2.1 .3 p-n junctions $\ldots \ldots \ldots \ldots$

2.1 .4 Electron beam induced current . . . . . . . . . . . . . . . . . . . . . . . . . . . . . . . . . . . . . .

2.1 .5 Heterojunctions $\ldots \ldots \ldots \ldots \ldots \ldots$

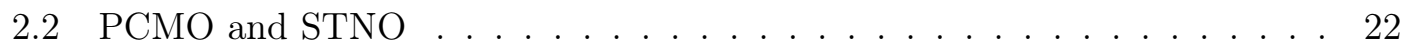

2.2 .1 Perovskite structure $\ldots \ldots \ldots \ldots \ldots$

2.2 .2 Polarons . . . . . . . . . . . . . . . . . . . . . . . . . . 23

2.2 .3 Niobium doped strontium titanate . . . . . . . . . . . . . . . . 23

2.2 .4 Calcium doped praseodymium manganite . . . . . . . . . . . . 24

2.2 .5 PCMO-STNO p-n junctions . . . . . . . . . . . . . . . . . . . 28

2.3 Electron microscopy $\ldots \ldots \ldots \ldots$

$2.3 .1 \quad$ Scanning electron microscopy $\ldots \ldots \ldots$. . . . . . . . . . 31

2.3 .2 (Scanning) transmission electron microscopy . . . . . . . . . . . . . 32

$2.3 .3 \quad$ Electron energy loss spectroscopy . . . . . . . . . . . . . . . . 33

2.3 .4 Energy filtered transmission electron microscopy . . . . . . . . . . 35

2.3 .5 Off-axis electron holography $\ldots \ldots \ldots \ldots$. . . . . . . . . . 36

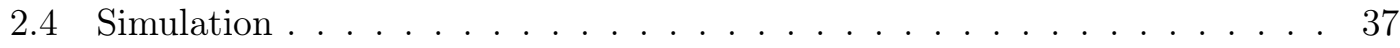

2.4 .1 Monte Carlo simulation in CASINO . . . . . . . . . . . . 37

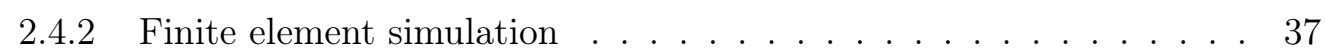

\begin{tabular}{lll}
\hline 3 & Experimental setup and methods & 39
\end{tabular}

$3.1 \quad$ Sample preparation . . . . . . . . . . . . . . . . . . . . . . . . . . . 39

$3.1 .1 \quad$ PCMO-STNO samples $\ldots \ldots \ldots$

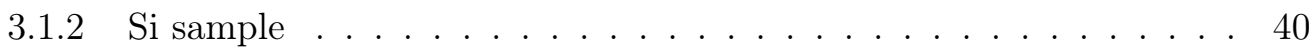

3.2 Focused Ion Beam . . . . . . . . . . . . . . . . . . . . . . . . . . 41

3.3 NanoSEM with cooling stage . . . . . . . . . . . . . . . . . . . 43

3.4 SEM EBIC setup . . . . . . . . . . . . . . . . . . . . . . . . . . . . . . 44

3.4 .1 EBIC preamplifier limitations . . . . . . . . . . . . . . 46

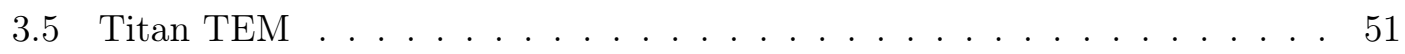

3.6 TEM EBIC setup $\ldots \ldots \ldots \ldots$

3.7 TEM holography setup $\ldots \ldots \ldots \ldots \ldots$. . . . . . . . . . . . . . . . . 53

3.8 Focused ion beam preparation procedures $\ldots \ldots \ldots \ldots$. . . . . . . . . 53

3.8 .1 Cross section lamellas for SEM-based EBIC . . . . . . . . . . . . 53

3.8 .2 Cross section lamellas for electron holography . . . . . . . . . . . 54

3.8 .3 Cross section lamellas for TEM-based EBIC . . . . . . . . . . . . . 54 
3.9 EBIC processing $\ldots \ldots \ldots \ldots \ldots$

$3.9 .1 \quad$ Quantification of SEM-based EBIC images. . . . . . . . . . . . . . 59

3.9 .2 Reconstruction of TEM-based EBIC profiles . . . . . . . . . . . . . . 59

4 Diffusion length estimation by low energy EBIC 61

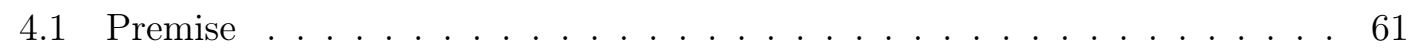

4.2 Methods . . . . . . . . . . . . . . . . . . . . . . . . . . . . . . . . 62

4.3 Results and discussion $\ldots \ldots \ldots$. . . . . . . . . . . . . . . . . . . . . . 63

4.4 Contributions . . . . . . . . . . . . . . . . . . . . . . . . . . . . . . . . . . . . . 64

5 Low energy scanning transmission electron beam induced current for nanoscale $\begin{array}{lr}\text { characterization of } p-n \text { junctions } & 65\end{array}$

5.1 Introduction . . . . . . . . . . . . . . . . . . . . 65

5.2 Methods . . . . . . . . . . . . . . . . . . . . . . . 67

5.3 Results and Discussion $\ldots \ldots \ldots \ldots$. . . . . . . . . . . . . . . 68

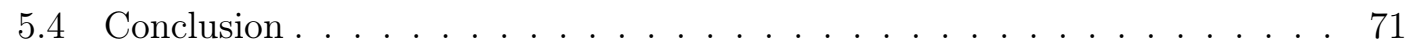

5.5 Acknowledgement . . . . . . . . . . . . . . . . . . . . . . . 71

5.6 Supporting Information $\ldots \ldots \ldots \ldots$. . . . . . . . . . . . . . 72

5.6 .1 Determination of the wedge lamella thickness gradient . . . . . . . 72

5.6 .2 EBIC map of the Si wedge . . . . . . . . . . . . . . . 73

5.6 .3 PCMO-STNO EBIC maxima at $15 \mathrm{kV} \ldots \ldots \ldots$. . . . . . . . 74

5.7 Contributions . . . . . . . . . . . . . . . . . . . . . . . . . . . 75

6 Nanometer range diffusion length in a polaronic perovskite solar cell measured $\begin{array}{ll}\text { by scanning transmission EBIC } & 77\end{array}$

6.1 Abstract . . . . . . . . . . . . . . . . . . . . . . . 77

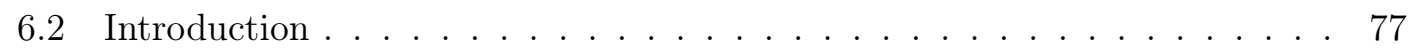

6.3 Results and Discussion $\ldots \ldots \ldots \ldots$. . . . . . . . . . . . . . . 80

6.3 .1 Decay length $\ldots \ldots \ldots$. . . . . . . . . . . . . . . . 81

6.3 .2 EBIC maxima $\ldots \ldots \ldots \ldots . \ldots \ldots$

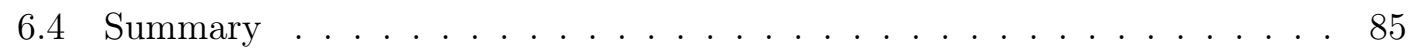

6.5 Methods . . . . . . . . . . . . . . . . . . . . . . . . . . . . . . 85

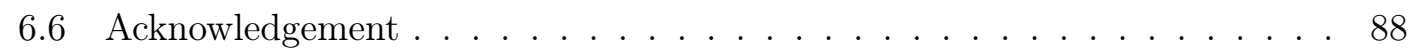

6.7 Contributions . . . . . . . . . . . . . . . . . . . . . . . . . . . . 88

7 EBIC temperature behaviour, space charge region and modeling of a PCMO$\begin{array}{lc}\text { STNO p-n heterojunction } & 89\end{array}$

7.1 Premise $\ldots \ldots \ldots \ldots \ldots$. . . . . . . . . . . . . . . . . . . . . . . . 89

7.2 Methods . . . . . . . . . . . . . . . . . . . . . . . . 89

7.3 Experimental results $\ldots \ldots \ldots \ldots$. . . . . . . . . . . . . . . . . . 93

7.3 .1 Room temperature STEBIC experiments. . . . . . . . . . . . . . 94

$7.3 .2 \quad$ Low temperature STEBIC experiments . . . . . . . . . . . . . . . . . . . . . . . . . . . . . . .

7.3 .3 TEM holography experiments . . . . . . . . . . . . . . . . . . 102

7.4 Modeling . . . . . . . . . . . . . . . . . . . . . . . . . . . . . . . . . . . 104

$7.4 .1 \quad$ Collection efficiency model . . . . . . . . . . . . . . . . . . . . 104

7.4 .2 COMSOL model . . . . . . . . . . . . . . . . . . . . . . 105

7.5 Contributions $\ldots \ldots \ldots \ldots$. . . . . . . . . . . . . . . . . . . . . . 107 
$\begin{array}{lll}8 & \text { Discussion } & 109\end{array}$

8.1 Sample preparation . . . . . . . . . . . . . . . . . . . . . . . . 109

$8.2 \quad$ EBIC measurement . . . . . . . . . . . . . . . . . . . . . . . . . . . . . 110

8.3 EBIC processing $\ldots \ldots \ldots \ldots 112$

8.4 Correction of EBIC preamplifier limitations . . . . . . . . . . . . . . 113

8.5 CASINO simulation $\ldots \ldots \ldots \ldots \ldots \ldots$

8.6 COMSOL simulation . . . . . . . . . . . . . . . . . . . . . . . . . . 118

8.7 Summarizing discussion $\ldots \ldots \ldots \ldots$. . . . . . . . . . . . . . . . . . . . . 124

$8.7 .1 \quad$ Influence of the generation volume . . . . . . . . . . . . . . . . 124

8.7 .2 Impact of the sample surface $(\mathrm{s})$. . . . . . . . . . . . . . . . . 125

8.7 .3 Effect of the space charge region . . . . . . . . . . . . . 127

8.7 .4 Determining the diffusion length . . . . . . . . . . . . . . . 127

8.7 .5 Simultaneous EBIC and EELS measurements . . . . . . . . . . . . 129

8.7 .6 Temperature influence . . . . . . . . . . . . . . . . . . 129

8.8 Outlook . . . . . . . . . . . . . . . . . . . . . . . . 130

8.8 .1 Experimental apparatus . . . . . . . . . . . . . . . . . 130

8.8 .2 Modeling . . . . . . . . . . . . . . . . . . . . . . . . . 131

$8.8 .3 \quad$ Sample system $\ldots \ldots \ldots \ldots$. . . . . . . . . . . . . . . 132

9 Summary 133

\begin{tabular}{ll}
\hline Bibliography & 135
\end{tabular} 



\section{Chapter 1}

\section{Introduction}

The general physical process of photovoltaics is the conversion of external optical excitation, such as by sunlight, to electric power, leading to its attractive status in the renewable energy field. Although photovoltaic devices have considerably advanced in the last decades, conventional devices based e.g. on silicon are still subject to the fundamental Shockley-Queisser limit [1]. Essentially, this consideration explains that only irradiation with the energy of the device's semiconductor bandgap can be effectively converted, leading to a fundamental conversion efficiency limit, which is $32 \%$ of the solar spectrum for Si devices. Light of lower energy transmits the device, whereas light of higher energy is initially absorbed, but the additional energy compared to the bandgap energy is lost due to rapid (picosecond range) thermalization of excited charge carriers to the conduction band edge. A feasible way to circumvent this limit is the use of tandem solar cells, which feature absorber materials with different bandgaps, therefore increasing the spectral range of usable light absorption 24 . Still, devices using a single absorption layer are considerably easier to prepare than tandem devices. Thus, it is desirable to find a material capable of harvesting a broad optical spectral range.

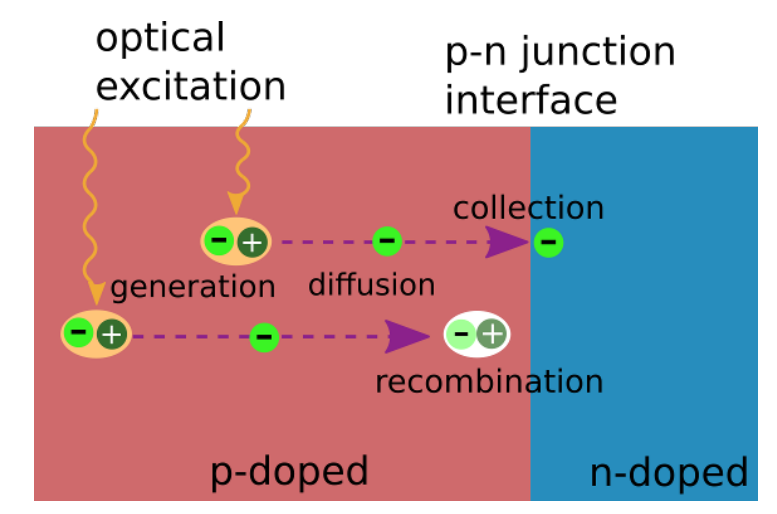

Figure 1.1: Sketch of principle processes in a p-n junction. Electrons and holes are optically excited pairwise, diffuse through the junction and can either be collected by the junction interface or recombine with a charge carrier of the other type. Note that only excess minority charge carriers are shown here, i.e. electrons in the p-doped region.

The energy conversion process by external energy influx in a p-n junction, which is the basic component of a photovoltaic device, is shown in Fig. 1.1 The p-n junction is brought out of thermal equilibrium by the external energy, generating additional (excess) electrons and holes pairwise. Excess charge carriers are subject to diffusion through the sample, collection at the junction interface and recombination of an electron and a hole. Recombination is the main process limiting the efficiency of a p-n junction, while another 
one is the rapid thermalization of excess charge carriers.

A promising way to suppress both is using charge carriers of polaron nature. Polarons are quasiparticles consisting of an electron or a defect electron (hole) coupled to a lattice distortion of the material. Changing the state of a polaron is generally more energy expensive than changing the state of a pure electron or hole, which leads to a decreased recombination probability as well as suppressed thermalization ('hot' charge carriers).

Manganite perovskites are a material class prominently featuring dominant contributions to charge carrier dynamics by polaron charge carriers. In general, they exhibit strong correlations between different material degrees of freedom [5], making them attractive for basic research of these correlations as well as for a variety of applications such as switching processes in resistive random-access memory [6], catalytic behavior for electrochemistry [7], thermoelectrics [8] and photovoltaics [9]. Significant effort has been put into basic research of this material system, for example in the multi-disciplinary DFG collaborative research center (CRC) 1073 at Georg August University Göttingen. A notable example of a manganite perovskite is $\operatorname{Pr}_{1-x} \mathrm{Ca}_{x} \mathrm{MnO}_{3}$ (PCMO), which features several charge and orbital ordered phases with different electric and magnetic states in its phase diagram, depending on Ca doping $x$ and temperature [10].

$\mathrm{P}-\mathrm{n}$ junctions using a p-doped PCMO layer on an n-doped $\mathrm{SrTi}_{1-y} \mathrm{Nb}_{y} \mathrm{O}_{3}$ (STNO) substrate have proven rectifying behavior, an essential factor for photovoltaics [11], and optical excitation processes in these junctions have been studied thoroughly since $[9,12$ 14]. A prominent feature for photovoltaics is the large spectral range of absorbed external optical irradiation, spanning from the near-infrared to the ultraviolet range, which is attributed to polaronic charge carriers [15].

A downside of polaronic charge carriers in manganite perovskites is their considerably smaller mobility (e.g. $0.02 \frac{\mathrm{cm}^{2}}{\mathrm{Vs}}$ for PCMO $[12 \mid$ ) compared to e.g. electrons and holes in Si (1450 and $500 \frac{\mathrm{cm}^{2}}{\mathrm{Vs}_{\mathrm{s}}}$ at room temperature, respectively $[16]$ ). Therefore, the diffusion length, which is directly connected to the mobility and the excess charge carrier lifetime, is expected to be much smaller (in the nanometer range) than in Si (up to the millimeter range) [12, 16. The knowledge of the diffusion length is thus critical in understanding the performance and physical processes of photovoltaic devices, especially for the complex behavior of manganite perovskites. For example, it can serve as a means of showing the influence of the low temperature charge ordered phase in PCMO, where an increased lifetime compared to the room temperature unordered phase was reported 13.

A classic way of measuring diffusion lengths up to the order of $10 \mu \mathrm{m}$ is using the electron beam induced current (EBIC) method, where a focused electron beam is scanned across a charge separating junction - e.g. a p-n junction - within the environment of an electron microscope, with several typical geometries available depending on the actual scale of the diffusion length [17, 18]. Like optical excitation, pairwise electron and hole excitation is also possible by impinging high energy electrons, which has the advantage of better spatial resolution.

However, the impinging electron beam creates a cascade of excitations within the sample, forming an extended distribution of generated charge carriers, which is called generation volume [19]. The extension of this volume heavily depends on the initial energy of the impinging electrons, which is characterized by the acceleration voltage used for the electron beam in the microscope. While helping to obtain a good signal-to-noise ratio in EBIC maps, and being useful to probe different sample depths in the plan view geometry [20], the extended generation volume severely limits the spatial resolution in the cross section 
geometry (alternatively called normal collector geometry in some literature), which is suitable to measure diffusion lengths below $1 \mu \mathrm{m}[21,22$. As the extension of the generation volume can be in the $200 \mathrm{~nm}$ range even for $5 \mathrm{kV}$, and even larger for increasing acceleration voltage, it is impossible to directly measure the diffusion length from EBIC profile decay from the charge collecting interface. Even using small acceleration voltages, the extension is comparable to the diffusion length as well as the space charge region around the charge collecting interface, which exhibits a built-in electric field due to p-n junction formation. In most common EBIC models, it is assumed that the diffusion length is much longer than the extension of space charge region and generation volume. Consequently, these models fail in systems like PCMO-STNO.

In this work, the approach to reduce the active sample thickness in cross section EBIC experiments in order to confine the size of the generation volume is explored using focused ion beam prepared lamellas from macroscopic p-n junction samples. This method is called scanning transmission EBIC (STEBIC), as part of the electron beam transmits the sample. This method is conducted in a scanning electron microscope (SEM) as well as in a scanning transmission electron microscope (STEM), where higher acceleration voltages are available which reduce the size of the generation volume to quasi-point-like conditions.

The lamella surfaces severely impact STEBIC experiments because of increased excess charge carrier recombination compared to the sample bulk, and surface layers damaged by ion irradiation during the preparation process. As a control parameter for surface effects, the lamella thickness is reduced gradually in the used samples by using a wedge shape geometry and measuring its thickness gradient. This approach allows to substantiate the surface effects, and distinguishing between them.

Modeling is required to distinguish between the different effects which make up EBIC profiles. As analytical models usually fail in the case where space charge region, generation volume and diffusion length are all of similar size, different roads are taken in this work. The generation volume can be simulated by Monte Carlo methods, and used to model the lamella thickness and acceleration voltage dependent generation volume effect on STEBIC profiles. A more advanced simulation procedure is simulating the behavior of the p-n junction by the finite element method, providing numerical solutions to essential semiconductor equations.

After a description of the theoretical background and experimental as well as modeling methods, the results of this work are presented in four chapters. A first estimation of the diffusion length of PCMO and STNO is presented in Ch. 4. based on results published in [12]. The STEBIC method with a controlled lamella thickness is explored in low electron beam energy conditions in $\mathrm{Ch}$. 5, as published in [23]. In $\mathrm{Ch}$. 6, experiments are moved to high energy conditions in a STEM. The low temperature PCMO phase is investigated in Ch. 7, combined with studies of the space charge region by means of electron holography, and the implementation of two methods for STEBIC data modeling. Finally, discussion of the results is deepened in Ch. 8 .

The author's contributions are specified at the end of each of chapters 4, 5, 6, and 7. All other chapters are solely the work of the author. 



\section{Chapter 2}

\section{Theory}

In this chapter, theoretical basics of the thesis are explained, starting with p-n junction and EBIC principles, continuing with the examined material system and concluding with the used experimental and theoretical methods.

\subsection{Photovoltaics in a $\mathrm{p}-\mathrm{n}$ junction}

\subsubsection{Excess carriers in semiconductors}

In semiconductors, the concentration of free charge carriers, i.e. electrons and defect electrons, also known as holes, is given by the atomic structure and can be influenced by doping. Additional free electrons and holes continuously appear and annihilate in pairs even under thermal equilibrium conditions. They always appear in pairs, as an electron from the valence band is excited into the conduction band, leaving behind a hole. Thus they do not influence the total net charge. The rate of annihilation events, called recombination, is always equal to the generation rate $G_{e q}$ in thermal equilibrium. It is proportional to the total charge carrier concentration $p$ or $n$ and limited by the respective charge carrier lifetimes $\tau_{p}$ and $\tau_{n}$. Hence, 24.

$$
G_{e q}=\frac{p}{\tau_{p}}=\frac{n}{\tau_{n}}
$$

Furthermore, it is possible to generate pairs of excess charge carriers via an external energy source such as a light, an electron or a neutron beam. This is a non-equilibrium condition. In the simplest case, the generation process requires an energy gain of the electron of the amount of the bandgap energy $E_{g}$. In reality, the situation is more complicated, but a universal law for semiconductors with many different types of penetrating radiation has been found by Klein [25], including an estimate of phononic processes. In this model, the electron hole pair energy $E_{e} h$ only depends on the bandgap energy $E_{g}$ and given as

$$
E_{e h}=\frac{14}{5} E_{g}+E_{p h},
$$

with $E_{p h}$ being the phononic energy loss in the excitation process, which is approximately constant with $0.5 \mathrm{eV} \leq E_{p h} \leq 1.0 \mathrm{eV}$.

The generation rate in the non-equilibrium case, called $G$, may be spatially inhomogeneous, depending on the source type. However, in a homogeneous semiconductor, the recombination rate $U$ may still be described by spatially independent lifetimes $\tau_{p}$ and $\tau_{n}$ 24 .

Including processes of generation and recombination, the general continuity equations 
for hole and electron current densities (current per area) $\vec{J}_{p}$ and $\vec{J}_{n}$ can be written independently as 16

$$
\begin{aligned}
-\frac{1}{e} \vec{\nabla} \cdot \vec{J}_{p}+G_{p}-U_{p} & =\frac{\partial p}{\partial t} \\
\frac{1}{e} \vec{\nabla} \cdot \vec{J}_{n}+G_{n}-U_{n} & =\frac{\partial n}{\partial t}
\end{aligned}
$$

All current densities $J$ may be transformed to currents $I$ by multiplying with the contact area. In stationary considerations, the time derivatives on the right hand side may be set as zero. The current densities can be written as expressions of the charge carrier concentrations $p$ and $n$ [16]:

$$
\begin{aligned}
& \vec{J}_{p}:=\vec{J}_{p, \text { diff }}+\vec{J}_{p, \text { drift }}=-e D_{p} \vec{\nabla} p+e \mu_{p} p \vec{E} \\
& \vec{J}_{n}:=\vec{J}_{n, \text { diff }}+\vec{J}_{n, \text { drift }}=e D_{n} \vec{\nabla} n+e \mu_{n} n \vec{E},
\end{aligned}
$$

where $e$ is the elemental charge and $\vec{E}$ is the local electric field in the semiconductor, which is determined from the total charge density $\rho$ and the permittivity $\varepsilon$ of the material via the Poisson equation 16

$$
\vec{\nabla} \cdot \vec{E}=\frac{\rho}{\varepsilon} .
$$

The current can be separated into a diffusion and a drift current. While the diffusion currents are determined by the respective diffusion coefficients $D_{p}$ and $D_{n}$, the drift currents incorporate the respective drift mobilities $\mu_{p}$ and $\mu_{n}$. These quantities are connected via the Einstein relations

$$
D_{p}=\frac{\mu_{p} k_{B} T}{e} \text { and } D_{n}=\frac{\mu_{n} k_{B} T}{e}
$$

with Boltzmann constant $k_{B}$, temperature $T$ and elemental charge $e$.

They are valid for all systems following Boltzmann statistics, which means that the Fermi-Dirac statistics can be approximated as [16

$$
F(E, T)=\frac{1}{\exp \left(\frac{E-E_{F}}{k_{B} T}\right)+1} \approx \exp \left(-\frac{E-E_{F}}{k_{B} T}\right) .
$$

This approximation, called non-degeneracy condition, means that the band edges are far enough (usually $2 k_{B} T$ ) away from the Fermi level $E_{F}$. The Boltzmann approximation enables a simple solution of the charge carrier concentrations, which are generally defined by

$$
\begin{aligned}
p & =\int_{-\infty}^{E_{V}} D_{V} O S_{V}(E)(1-F(E, T)) \mathrm{d} E \\
n & =\int_{E_{C}}^{\infty} D_{C} O S_{C}(E) F(E, T) \mathrm{d} E .
\end{aligned}
$$

If the band edges of the conduction band $E_{C}$ and the valence band $E_{V}$ can be approximated as parabolic near the relevant wave vectors (constant effective mass approximation), the densities of states in the valence and the conduction band are

$$
D O S_{C}(E) \propto \sqrt{E-E_{C}} \text { and } \operatorname{DOS}_{V}(E) \propto \sqrt{E_{V}-E},
$$


which makes integration in equations 2.9 and 2.10 simple. In case of the Boltzmann equation, $n$ and $p$ become

$$
\begin{aligned}
& p=N_{V} \exp \left(-\frac{E_{F}-E_{V}}{k_{B} T}\right) \\
& n=N_{C} \exp \left(-\frac{E_{C}-E_{F}}{k_{B} T}\right)
\end{aligned}
$$

with the effective densities of states of the valence band $N_{V}$ and the conduction band $N_{C}$. With the three mentioned approximations, $N_{C}$ and $N_{V}$ are constants for one temperature and effective mass.

The approximation in equation 2.8 is not valid in degenerate semiconductors, where the Fermi energy is closer than $2 k_{B} T$ to a band edge, or even within the valence or conduction band. This happens if the charge carrier concentrations are close to or larger than $N_{V}$ or $N_{C}$ and causes the behaviour of the semiconductor to change to metallic behaviour, as the bandgap is not the limiting factor of thermal excitation anymore if the Fermi energy is within a band [16]. This condition can lead to effects like metallic conductance, non-temperature related charge carrier density, decreasing size of the bandgap or defect bands within the bandgap 16,26 . In the degenerate case, the exponential functions in equations 2.12 and 2.13 have to be replaced by the special Fermi-Dirac integral [16]

$$
F_{1 / 2}(x)=\frac{2}{\sqrt{\pi}} \int_{0}^{\infty} \frac{\sqrt{t}}{\exp (t-x)+1} \mathrm{~d} t
$$

$x$ being the argument of the exponentials. Furthermore, the Einstein relations (equation 2.7 are not valid anymore in the degenerate case and have to be replaced by more general relations dependent on the charge carrier concentrations and the Fermi energy:

$$
D_{p}=\frac{-\mu_{p} p}{e \frac{\mathrm{d} p}{\mathrm{~d} E_{F}}} \quad \text { and } \quad D_{n}=\frac{\mu_{n} n}{e \frac{\mathrm{d} n}{\mathrm{~d} E_{F}}} .
$$

Both the Fermi integral and the general Einstein relations do not in general allow simple solutions and require extended approximations or numerical treatment.

\subsubsection{Generation, recombination and diffusion length}

The general balance equation for the time-dependent generation and recombination of electron hole pairs is 27

$$
\frac{\mathrm{d} p}{\mathrm{~d} t}=\frac{\mathrm{d} n}{\mathrm{~d} t}=G_{\mathrm{tot}}-B \cdot p \cdot n
$$

with the total generation rate $G_{\text {tot }}$ and the recombination coefficient $B$, which - in general - may depend on $p$ and $n$. In case of non-equilibrium generation, it is useful to discriminate between the concentration of charge carriers $p_{e q}$ and $n_{e q}$ which are generated in thermal equilibrium and $\delta p$ and $\delta n$ which are additionally generated by the external energy source with generation rate $G$. Thus, $p=p_{e q}+\delta p$ and $n=n_{e q}+\delta n$. In the non-equilibrium stationary case $\left(\frac{\mathrm{d} p}{\mathrm{~d} t}=\frac{\mathrm{d} n}{\mathrm{~d} t}=0\right)$, Eq. 2.15 becomes

$$
G_{e q}+G-B \cdot p \cdot n=B p_{e q} n_{e q}+G-B\left(p_{e q}+\delta p\right)\left(n_{e q}+\delta n\right)=0 .
$$

Assuming $\delta p=\delta n$, i.e. pair-wise generation of electrons and holes, Eq. 2.16 can be rearranged to

$$
\frac{\delta p}{p_{e q}+n_{e q}}=\sqrt{\frac{G}{B\left(p_{e q}+n_{e q}\right)^{2}}+\frac{1}{4}}-\frac{1}{2},
$$


which can be written as

$$
\delta p^{*}=\sqrt{G^{*}+\frac{1}{4}}-\frac{1}{2}
$$

with the reduced generation rate $G^{*}:=\frac{G}{B\left(p_{e q}+n_{e q}\right)^{2}}$ and the reduced injection (excess charge carrier concentration) $\delta p^{*}:=\frac{\delta p}{p_{e q}+n_{e q}}$. This general relation of generation and injection allows separating the regimes of low $\left(\delta p^{*} \ll 1\right)$ and high $\left(\delta p^{*} \gg 1\right)$ injection. While the injection is linear to the generation in the low injection regime, they show a square root behaviour in the high injection regime, i.e. slower growth of the injection for very high generation. However, this behaviour is only strictly valid if $B$ is independent of the charge carrier concentrations, which is not the case for all recombination mechanisms, as explained in the following.

The bulk recombination rate $U$, as shown in equations 2.2 and 2.3 , depends on the dominant recombination mechanism in the semiconductor. The three most important models are radiative, Auger and Shockley-Read-Hall recombination [28]. For radiative (or bandto-band) recombination, an electron in the conduction band recombines directly with a hole in the valence band by sending out a phonon with the bandgap energy $E_{g}$ (for direct semiconductors, otherwise a part of the energy is transferred to phonons). For Auger recombination, an electron or a hole is released which might leave the semiconductor or relax under energy transfer to a phonon. For Shockley-Read-Hall recombination, electrons or holes do not recombine directly from conduction to valence band edges, but in a two-step process via trap states in the bandgap. While radiative recombination is most common for direct semiconductors, Shockley-Read-Hall recombination often dominates in indirect semiconductors. Auger recombination is dominant for materials with high doping levels. The recombination rates of excess charge carriers for the radiative and Auger processes are given by

$$
\begin{aligned}
U_{\text {rad }} & =B \cdot\left(p \cdot n-n_{i}^{2}\right) \\
U_{\text {Auger }} & =\left(B_{p} p+B_{n} n\right) \cdot\left(p \cdot n-n_{i}^{2}\right)
\end{aligned}
$$

The Shockley-Read-Hall recombination rate is more complicated, depending on the number of traps and might include integration over the bandgap if many states are involved. In the following, it is assumed that the dominant recombination process, no matter what its actual nature is, can be written in the form of equation 2.18, meaning that $B$ could very well depend on $p$ and $n$. As mentioned before, recombination may also be written in terms of a bulk charge carrier lifetime $\tau$ :

$$
U_{r a d, p}=\frac{\delta p}{\tau_{p}} \quad \text { or } \quad U_{r a d, n}=\frac{\delta n}{\tau_{n}}
$$

Using the diffusion coefficients $D_{p}$ and $D_{n}$, the lifetime can be converted to a diffusion length $L$. One may write for electrons and for holes 16

$$
L_{p}=\sqrt{D_{p} \tau_{p}} \text { or } \quad L_{n}=\sqrt{D_{n} \tau_{n}} .
$$

Connecting equations 2.18, 2.20 and 2.21, and rewriting the excess concentrations as $\delta p=p-p_{e q}$ and $\delta n=n-n_{e q}$, the diffusion length may also be written as

$$
L_{p}=\sqrt{\frac{D_{p}\left(p-p_{e q}\right)}{B\left(p n-p_{e q} n_{e q}\right)}} \quad \text { or } \quad L_{n}=\sqrt{\frac{D_{n}\left(n-n_{e q}\right)}{B\left(p n-p_{e q} n_{e q}\right)}} .
$$


In doped semiconductors, the doping concentration $N_{A}$ (acceptors in p-doping) or $N_{D}$ (donors in n-doping) is usually much higher than the intrinsic charge carrier concentration $n_{i}$. Thus $p \approx N_{A}$ in p-doped (p-type) and $n \approx N_{D}$ in n-doped (n-type) semiconductors. The law of mass action requires that in thermal equilibrium 24

$$
n \cdot p=n_{i}^{2}
$$

This leads to the concept of majority and minority carriers: If the concentration of one type of charge carriers is much higher than $n_{i}$, the other one must be lower by the same order of magnitude. The former is thus called majority charge carrier, the latter minority charge carrier.

In cases where $p \approx p_{e q}=N_{A}$, i.e. p-doped material under low injection conditions, the electron (minority charge carrier) diffusion length can be approximated as

$$
L_{n, p} \approx \sqrt{\frac{D_{n}}{B N_{A}}} .
$$

For n-doped material $\left(n \approx N_{D}\right)$, the hole diffusion length thus becomes

$$
L_{p, n} \approx \sqrt{\frac{D_{p}}{B N_{D}}} .
$$

Another excess carrier recombination mechanism can be found at sample surfaces, where a larger number of defects tends to be present, leading to increased Shockley-Read-Hall recombination as compared to the bulk of the sample. The recombination mechanism can be formulated as a boundary condition of the currents $\vec{J}_{p}$ and $\vec{J}_{n}$, as defined by equations 2.4 and 2.5 , through a surface by using the vector $\vec{\eta}$ which is defined as normal to the surface 24]:

$$
\vec{\eta} \cdot \frac{1}{e} \vec{J}_{p}=s \cdot\left(p-p_{e q}\right) \quad \text { or } \quad \vec{\eta} \cdot \frac{-1}{e} \vec{J}_{n}=s \cdot\left(n-n_{e q}\right)
$$

with the surface recombination velocity $s$ as a parameter. The higher $s$, the stronger the effect of surface recombination is. The surface recombination velocity depends on the used material as well as the preparation procedure of the sample. For example, ion beam preparation may severely increase the velocity [29]. Dangling bonds at the surface favor surface recombination, which may be saturated to decrease the effect. A typical saturation procedure for silicon material is hydrogen passivation [30]. Surface defects may also form electric fields at the surface, which can be described as a shift of the Fermi level compared to the bulk level, called Fermi level pinning [31].

\subsection{3 p-n junctions}

Combining a p-doped and an n-doped semiconductor leads to a concentration gradient in valence band holes and conduction band electrons. Described by the concept of electrochemical potential (typically referred to as Fermi level in semiconductor literature), there is a difference between the potential levels on the p-doped and the n-doped side. To reach an equilibrium state, the Fermi level must align, i.e. be constant throughout the whole structure. This leads to a diffusion current of the majority carriers to the other side. In turn, this shift of charge carriers leads to the formation of a space charge near the $\mathrm{p}$-n 
interface. According to the Poisson equation 2.6, the spatially fixed charge is connected to a difference in electronic potential $V$, and thus an electric field in the charged region. In the case of equilibrium Fermi level, a fixed potential difference between the p- and the n-doped region, called diffusion voltage $V_{D}$. It can also be described by the difference in the Fermi level dependent work functions $W_{p}, W_{n}$ (see Fig. 2.1) on both sides:

$$
V_{D}=\frac{W_{p}-W_{n}}{e}
$$

The work function is the energy electrons at the Fermi level have to gain in order to leave a material, i.e. reach the vacuum level $E_{v a c}$. It is connected to the electron affinity $\chi$, which is the energy electrons at the conduction band edge $E_{C}$ have to gain in order to reach the vacuum level [16]:

$$
W=\chi+\left(E_{C}-E_{F}\right)
$$

In contrast to $W, \chi$ is a constant for a given material, independent of the doping level and thus Fermi level position, at least for a non-degenerate material (see below for a description where this is not the case). For the geometry of a p-n junction plane

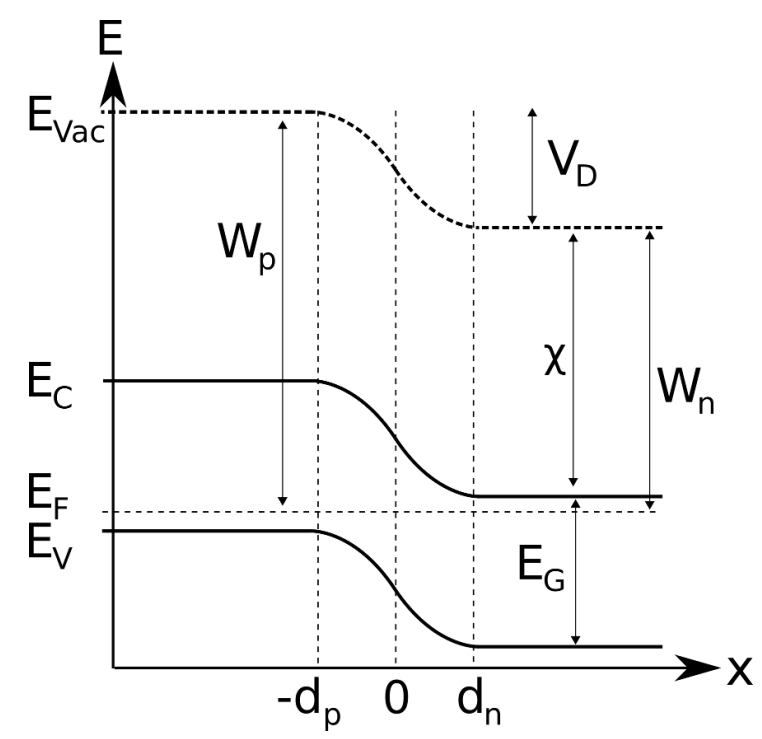

Figure 2.1: Sketched band diagram of a p-n homojunction in equilibrium. The shown band edges are the conduction band edge $E_{C}$ and the valence band edge $E_{V}$. The vacuum level $E_{v a c}$ and the work function $W$ follow the same shape as $E_{C}$ and $E_{V}$. Also shown are the constant electron affinity $\chi$, diffusion voltage $V_{D}$, Fermi energy $E_{F}$, p-n junction interface at $x=0$ and the edges of the space charge region at $x=-d_{p}$ and $x=d_{n}$ where the band bending vanishes.

separating two infinite half-spaces, a one-dimensional Poisson equation may be defined from Eq. 2.6 24]:

$$
\frac{d E}{d x}=-\frac{d^{2} V}{d x^{2}}=e \cdot \frac{p-n+C_{i o n}}{\varepsilon}
$$

with

$$
C_{\text {ion }}(x)=\left\{\begin{array}{ll}
-N_{A}, & \text { p-doped region } \\
N_{D}, & \text { n-doped region }
\end{array} .\right.
$$

We may now describe a simple solution of the Poisson equation (Eq. 2.29 known as box approximation. In this approximation the space charge region is assumed to be 
completely depleted of mobile charge carriers $(p=n=0)$ and is confined by sharp borders. For convenience, the $\mathrm{p}-\mathrm{n}$ junction plane shall be located at $x=0$ (see Fig. 2.1). The p-doped region shall be at $x<0$ and the $\mathrm{n}$-doped region at $x>0$, with the space charge region borders at $x=-d_{p}$ and $x=d_{n}$. Thus the total charge concentration profile $\rho(x)$ perpendicular to the $\mathrm{p}-\mathrm{n}$ junction plane is

$$
\rho(x)= \begin{cases}0, & x \leq-d_{p} \\ -e N_{A}, & -d_{p}<x \leq 0 \\ e N_{D}, & 0<x \leq d_{n} \\ 0, & x>d_{n}\end{cases}
$$

The permittivity $\varepsilon$ may also be region-dependent, jumping at $x=0$ from $\varepsilon_{p}$ to $\varepsilon_{n}$ in case of a heterojunction. Region-wise integration of Eq. 2.29 with the condition

$$
V_{n}(\infty)-V_{p}(-\infty)=V_{D}
$$

leads to 28

$$
V(x)= \begin{cases}-V_{i}, & x \leq-d_{p} \\ -V_{i}+\frac{e N_{A}}{2 \varepsilon_{p}}\left(x+d_{p}\right)^{2}, & -d_{p}<x \leq 0 \\ -V_{i}+V_{D}-\frac{e N_{D}}{2 \varepsilon_{n}}\left(d_{n}-x\right)^{2}, & 0<x \leq d_{n} \\ -V_{i}+V_{D}, & x>d_{n}\end{cases}
$$

including the arbitrary initial potential in the p-doped region outside the space charge region, $-V_{i}$. Continuity of the potential at $x=0$ requires that

$$
\frac{e N_{A}}{2 \varepsilon_{p}} d_{p}^{2}=V_{D}-\frac{e N_{D}}{2 \varepsilon_{n}} d_{n}^{2}
$$

while continuity of the electric displacement field $D=\varepsilon E=-\varepsilon \frac{d V}{d x}$ requires

$$
\frac{N_{A}}{\varepsilon_{p}} d_{p}=\frac{N_{D}}{\varepsilon_{n}} d_{n}
$$

The latter enables the calculation of $d_{p}$ and $d_{n}$ from the doping concentrations for a homojunction:

$$
\begin{aligned}
d_{p} & =\sqrt{\frac{2 \varepsilon_{p} V_{D}}{e} \frac{N_{D}}{N_{A}\left(N_{A}+N_{D}\right)}} \\
d_{n} & =\sqrt{\frac{2 \varepsilon_{n} V_{D}}{e} \frac{N_{A}}{N_{D}\left(N_{A}+N_{D}\right)}} .
\end{aligned}
$$

If $d_{p}$ and $d_{n}$ are not equal, the fractions $V_{D, p}$ and $V_{D, n}$ of $V_{D}=V_{D, p}+V_{D, n}$ dropping over the $\mathrm{p}$ and $\mathrm{n}$ parts of the space charge region are also asymmetric, and given by 32

$$
V_{D, p}=\frac{N_{A} x_{p}^{2}}{2 \varepsilon_{p}} \quad \text { and } \quad V_{D, n}=\frac{N_{D} x_{n}^{2}}{2 \varepsilon_{n}}
$$

leading to

$$
\frac{V_{D, n}}{V_{D, p}}=\frac{N_{A} \varepsilon_{p}}{N_{D} \varepsilon_{n}}
$$


which is also valid in cases where the box approximation does not hold.

Using equations 2.12 and 2.13 , the concentration profile of electrons and holes through the space region can be calculated from the band diagram (Fig. 2.1). At the crossing point of $n$ and $p$, the intrinsic charge carrier concentration $n_{i}$ of the material is reached. Note that this point, called intrinsic point $x_{i}$ is not always at the interface from p- to n-doped region $(x=0)$. In case of asymmetric doping, i.e. different doping on $\mathrm{p}$ - and n-side, the intrinsic point moves into the lower doped region [33]. This effect is demonstrated for a calculation based on Eq. 2.30 in Fig. 2.2 .

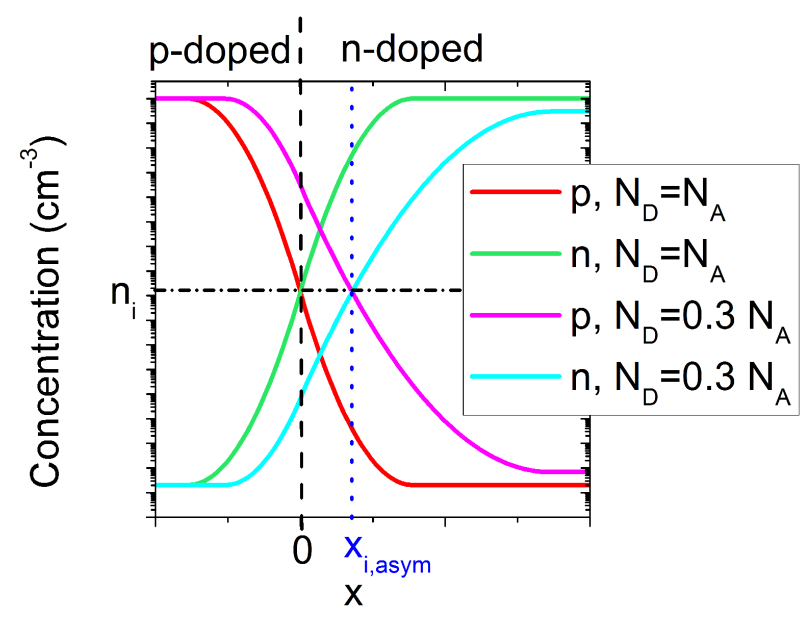

Figure 2.2: Electron and hole concentration profiles in the space charge region of a p-n homojunction, as calculated from Eq. 2.12 and 2.13 using the box approximation (Eq. 2.30). The space charge region ends where the concentrations become flat. Two cases are shown, one for symmetric and one for asymmetric doping, with reduced n-doping. The intrinsic point $x_{i}$, where $n=p=n_{i}$, is moved away from $x=0$, where doping changes abruptly, into the lower doped region in the second case.

To check the validity of the space charge region model, it is instructive to compare $d_{p}$ and $d_{n}$ to the Debye length $L_{D y}$, which is defined by [16]

$$
L_{D y}=\sqrt{\frac{\varepsilon k_{B} T}{e^{2} N}}
$$

with the general doping concentration $N$. Local charges in a semiconductor, e.g. impurities, may perturb the regular charge distribution of the material, but the range of the perturbation is limited as the additional charge is screened by the neighboring atoms. The Debye length, also known as screening length, is the decay length of the perturbation. If the space charge region is on the same scale or even smaller than the Debye length, it is generally not valid to use the box approximation 2.30 to treat the band bending in the space charge region, and the Poisson equation 2.29 is not sensitive to the abrupt change in doping in this region.

Four types of charge carrier currents occur in a p-n junction, which have been defined in eq. 2.4 and 2.5 . The diffusion current densities $J_{p, \text { diff }}$ and $J_{n, \text { diff }}$ are from charge carriers diffusing to the junction side of smaller concentration, where they recombine. They are thus also called recombination currents. On the other hand, the drift current densities $J_{p, \text { drift }}$ and $J_{n, \text { drift }}$ are from charge carriers following the electric field in the 
space charge region, being separated in the process. Generated excess charge carriers can be harvested by these processes, therefore they are called generation currents. In a p-n junction in thermal equilibrium, both currents balance out simultaneuosly for electrons and holes:

$$
J_{p, n}^{\text {diff }}=J_{p, n}^{\text {drift }} \propto \exp \left(-\frac{e V_{D}}{k_{B} T}\right)
$$

If a bias voltage $U$ is applied to the p-n junction (positive potential to the $\mathrm{p}$ side and negative to the n-side), equations 2.27, 2.31 and 2.32 have to be adjusted by substituting $V_{D}$ with $V_{D}-U$. Therefore, the total potential drop over the junction, which is also the diffusion barrier, reduces from $V_{D}$ to $V_{D}-U$ with a positive applied voltage $U$. This causes an imbalance between drift and diffusion currents: While the drift currents remain unchanged, the diffusion currents are modified from 2.35 to 24

$$
J_{p, n}^{\text {diff }} \propto \exp \left(-\frac{e\left(V_{D}-U\right)}{k_{B} T}\right)=J_{p, n}^{\text {drift }} \exp \left(\frac{e U}{k_{B} T}\right) .
$$

All four currents add up to a total current with the density

$$
J=J_{p}^{\text {diff }}-J_{p}^{\text {drift }}+J_{n}^{\text {diff }}-J_{n}^{\text {drift }}=J_{0}\left(\exp \left(\frac{e U}{k_{B} T}\right)-1\right)
$$

with the saturation current density $J_{0}=J_{p}^{\text {drift }}+J_{n}^{\text {drift }}$. This is known as the ideal Shockley model for a $J(U)$ characteristic [34].

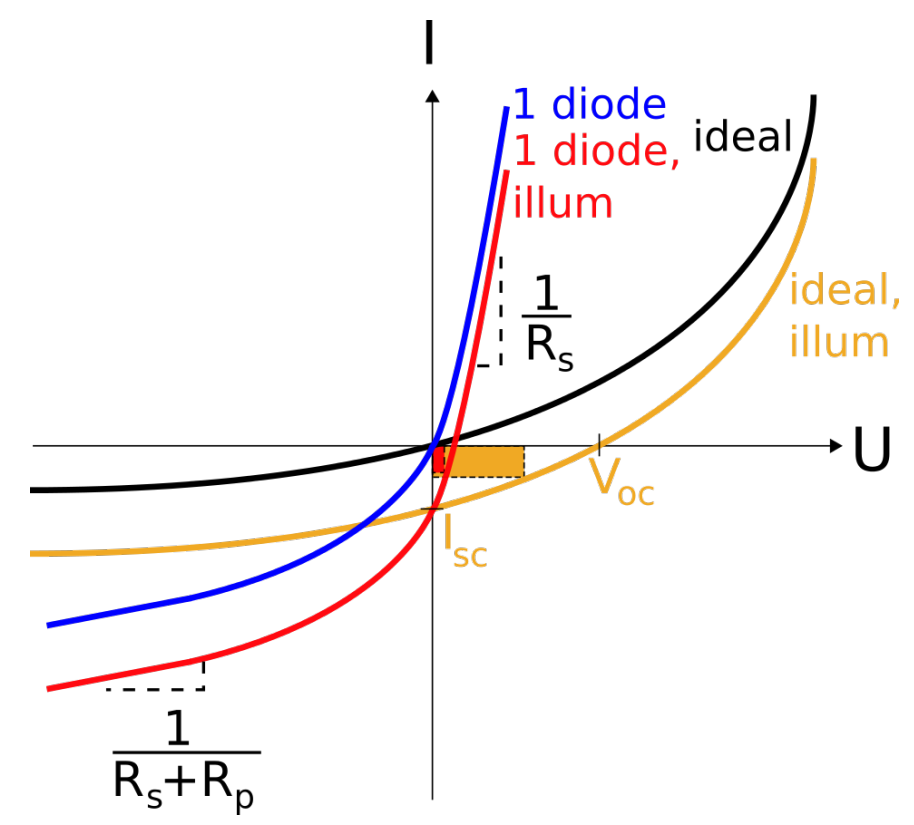

Figure 2.3: Shockley model for $I(U)$ characteristics, sketched for the ideal (equation 2.36) and the one diode model (equation 2.37) with and without illumination. Also shown are short circuit current $I_{s c}$, open circuit voltage $V_{o c}$ for the ideal model with illumination, curve slope dependent on the serial and parallel resistances $R_{s}$ and $R_{p}$ as well as the fill factor $F F$ (boxes), which is much smaller for the one diode model with finite resistances because of reduced $V_{o c}$.

Illumination of the p-n junction, as discussed in the beginning of this subsection, generates electron-hole pairs, which diffuse through the junction and are separated in the 
electric field of the space charge region. It can be shown that this leads to a shift of the ideal Shockley equation 2.36 by a negative term $J_{s c}$ which is called short-circuit current density (or $I_{s c}$ as current) as it also flows if no bias voltage is applied to the p-n junction. The shift of the $J(U)$ curve also yields a characteristic voltage $U_{o c}$ with $J\left(U_{o c}\right)=0$. In this case, the increased drift currents stemming from excess charge carriers are completely compensated by lowering the barrier $V_{D}-U$, thus also increasing the diffusion currents. This voltage is called open circuit voltage as no current is measured in this case if an amperemeter is connected to the biased p-n junction. It can also be measured directly by connecting a voltmeter to the illuminated p-n junction instead of an amperemeter and a voltmeter. Using an illuminated p-n junction as a solar cell, its maximum power output $P_{\max }=(I \cdot U)_{\max }$ can be calculated in the quadrant of positive $U$ and negative $I$. The fill factor

$$
F F=\frac{P_{\max }}{I_{s c} U_{o c}}
$$

characterizes the response of the solar cell to bias voltage.

The Shockley equation only describes the behaviour of ideal diodes. Real diodes, e.g. semiconductor based p-n junctions, require more complicated models. The one diode model is the simplest of these models and may be described in an equivalent circuit by adding two additional ohmic resistors to the circuit, $R_{p}$, and $R_{s}$, in parallel and in series to the diode, respectively. $R_{p}$ describes leakage through the diode, commonly called 'shunts', which may arise due to imperfections at the physical charge-separating interface. $R_{s}$ describes the sum of all resistances due to contact layers and connections such as cables.

The equivalent circuit of the one diode model is shown in Fig. 2.4. Another quantity in the one diode model is the ideality factor $n$ which is equal to 1 for an ideal diode and may increase up to 2 if Shockley-Read-Hall recombination is occuring in the space charge region of the considered semiconductor [35]. For even higher values, recombination at the charge separating interface may be enhanced by tunneling [36]. The modified Shockley equation for the one diode model including the short circuit current due to light illumination is thus (written for currents $I$ rather than current densities $J$ )

$$
I=I_{0}\left(\exp \left(\frac{e\left(U-I R_{S}\right)}{n k_{B} T}\right)-1\right)+\frac{U-I R_{S}}{R_{p}}-I_{s c} .
$$

For $R_{s} \rightarrow 0, R_{p} \rightarrow \infty$ and $n \rightarrow 1$, the model approaches the ideal Shockley model.

\subsubsection{Electron beam induced current}

The electron beam induced current (EBIC) method is an important characterization technique of devices exhibiting a photovoltaic effect [17]. Using a scanning electron hole pair source provided by a high energy (keV range) electron beam on a p-n junction or a similar charge separating device, the short circuit current $I_{s c}$ can be measured for each source position. By applying a bias voltage during the process, also arbitrary points of the $I(U)$ curve can be spatially mapped [37]. The resulting current map provides information about the local properties of a sample regarding the recombination behaviour of excess electrons and holes. For example, at electrically active defects electron-hole-pair recombination is typically greatly enhanced compared to bulk behaviour, showing them as regions of reduced intensity in EBIC images [38 40].

As the local generation of electron hole pairs raises the concentration of both charge 


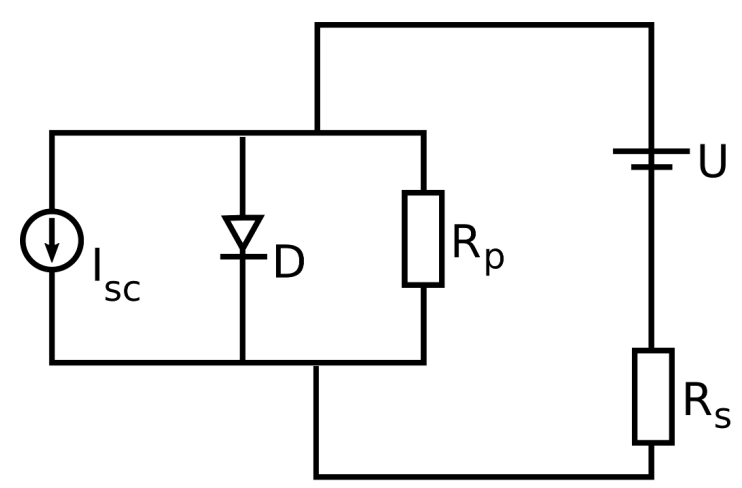

Figure 2.4: Equivalent circuit for a one diode model with ideality factor $n=1$ with a connected voltage source. The diode $D$ from the ideal Shockley model is complemented by a serial and a parallel resistor $R_{s}$ and $R_{p}$ to approach the behaviour of a real device. Furthermore, the short circuit current $I_{s c}$, flowing in reverse bias direction of the diode, is included, which arises from illumination of a p-n junction diode device.

carrier types equally, the minority charge carrier density is severely perturbed while the majority carriers are virtually unaffected unless high injection conditions apply. Thus the diffusion of minority charge carriers is the defining aspect. If a minority charge carrier crosses a p-n junction interface, it is not subject to this limited diffusion process anymore and contributes to the net current flowing through the sample. This can be described as collection by the $\mathrm{p}-\mathrm{n}$ junction. Minority charge carrier diffusion is generally limited by a finite diffusion length $L$, as defined in section 2.1.2. Thus, if the point of local generation is moved away from the p-n junction interface, the resulting EBIC signal will decrease at some point, and it will generally be highest when exciting directly at the interface.

The most direct way to measure the diffusion length is to record an EBIC profile across the $\mathrm{p}-\mathrm{n}$ junction interface in a cross-section geometry $21,22,41,42$. For the simplest case of a semi-infinite sample, a point-like space charge region and charge carrier source, and scanning perpendicular to the junction interface plane, the one dimensional diffusion problem, e.g. for excess electrons

$$
D_{n} \cdot \frac{\mathrm{d}^{2} \delta n}{\mathrm{~d} x^{2}}=\frac{\delta n}{\tau_{n}}
$$

which is connected to the diffusion length via $L_{n}=\sqrt{D_{n} \tau_{n}}$, can be solved directly using a method of image charges [43]. In this simple geometry, the p-n junction interface is placed at $x=0$, where charges are mirrored, and Eq. 2.38 is describing complete charge carrier motion in the half-infinite volume beyond the interface, i.e. the space charge region is neglected. Using the simplified generation rate $g$ (defined in $\frac{1}{\mathrm{~s}}$, i.e. ignoring the electron and hole charge carrier densities), the solution

$$
I_{\mathrm{EBIC}}(x)=e \cdot g \cdot \exp \left(-\frac{x}{L}\right)
$$

is obtained.

The extension of the space charge region on either side of the p-n junction interface can usually be estimated by equations 2.31 and 2.32. In this region, the simple model of equation 2.39 is not valid, as the electric field of the space charge region counteracts the diffusion process, i.e. the second summand of equations 2.4 and 2.5 , which enter into the continuity equations 2.2 and 2.3 . If the field is strong enough, which is the 
case for reasonably small space charge regions, it dominates over the diffusion and even the recombination terms. Consequently, in this model every charge carrier generated in or entering the space charge region, is collected and contributes to the EBIC. If the extension of the space charge region is significant compared to the diffusion length, but still smaller, the simple EBIC diffusion model 2.39 may be replaced by a collection function $f(x):=\frac{E B I C}{e \cdot g}[44,45]$, which may be defined region-wise as

$$
f(x)= \begin{cases}1, & -d_{p}<x \leq d_{n} \\ \exp \left(-\frac{|x|}{L}\right), & x \leq-d_{p}, x>d_{n}\end{cases}
$$
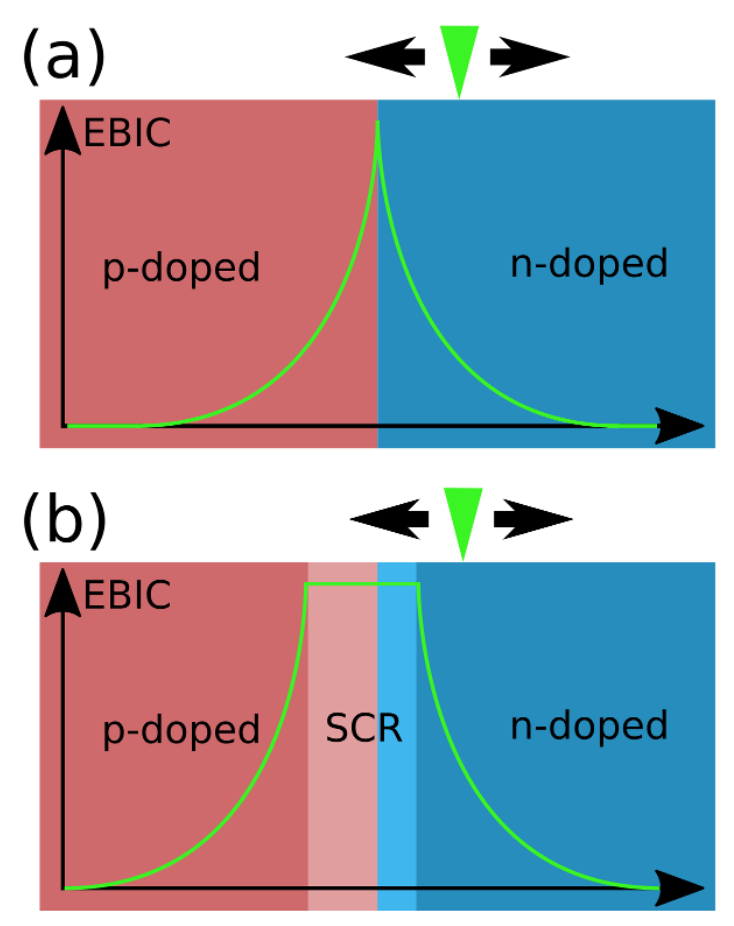

Figure 2.5: EBIC profiles when scanning an electron beam (green triangles) across a p-n junction in the case of point-like excitation, a semi-infinite sample and finite diffusion length. (a) for point-like space charge region (SCR), (b) for extended SCR

Another assumption for the simple EBIC model is a point-like extension of the electron hole pair source, compared to the diffusion length. However, high energy electrons impinging the sample are subject to multiple elastic as well as inelastic scattering processes. During these processes, the electron beam is broadened in the sample. For a sample of infinite thickness (in practice at least several $\mu \mathrm{m}$, the beam eventually forms a pear shaped volume inside the sample, where generation of electron hole pairs occurs, and which is called generation volume [19]. The size and shape of the generation volume depends on the initial energy of the impinging electrons, as well as the sample's mass density and atomic structure. The pear shape is best described for an amorphous structure (see [19]), for crystalline samples it should be noted that high energy electrons are subject to channeling [46], which should generally reduce the lateral (parallel to the impingement surface) generation volume size. If the extension of the generation volume is comparable to the diffusion length, it has to be included in proper EBIC profile modeling. This can 
be achieved by using a spatially dependent generation rate density $G(\vec{r})$ to describe the generation volume, which is connected to the spatial distribution $\varepsilon_{a}(\vec{r})$ of the absorbed energy density in the sample by the electron hole pair energy $E_{e h}$ defined in section 2.1.1 and the impinging beam current $I_{b}$ by

$$
G(\vec{r})=\frac{I_{b}}{e \cdot E_{e h}} \varepsilon_{a}(\vec{r})
$$

as each electron hole pair excited within this volume requires the energy $E_{\text {eh }}$ to form. A suitable EBIC model for an arbitrary impingement point $\vec{r}_{0}$ in the sample can be formulated as a convolution of generation volume and the three-dimensional collection function [41]:

$$
I_{\mathrm{EBIC}}\left(\vec{r}_{0}\right)=e \cdot G\left(\vec{r}_{0}\right) \times f\left(\vec{r}_{0}\right)=e \int_{\vec{r}} G\left(\vec{r}-\vec{r}_{0}\right) \cdot f(\vec{r}) d \vec{r}
$$

The three dimensional collection function can be deduced from the one dimensional collection function established in eq. 2.40. For an infinite sample with a p-n junction interface plane perpendicular to the x-direction at $x=0, f(\vec{r})$ has the trivial solution

$$
f(\vec{r})=f(x, y, z)=f(x) .
$$

If the sample has surfaces and/or contacts, the collection function generally has to be adjusted in the area outside the space charge region to account for their influence. For example, if the diffusion length is comparable to the distance of the contact to the p-n junction interface (i.e., the layer thickness), a solution for the one-dimensional collection function is given by Nichterwitz et al. [47]. Understanding the effects of surfaces on the collection function might be simplified by using the concept of an effective diffusion length $L_{e f f}$. If the one dimensional collection function modified for surface effects $\tilde{f}(x)$ can still be written as

$$
\tilde{f}(x)=\exp \left(-\frac{x}{L_{e f f}}\right)
$$

the general form given in equation 2.40 can still be used, but the diffusion length has to be adjusted to an effective value and might even be spatially dependent. If the distance $z$ from a point of local generation to the sample surface is comparable to the diffusion length, the effective diffusion length is generally smaller than the real diffusion length $L$, as the diffusion process is hindered by recombination at surface trap states, which may be described by a finite surface recombination velocity $s$ (see section 2.1.2).

The problem may be described within the approximation of homogeneous generation $G(\vec{r})=G$ by the depth-dependent excess charge carrier concentration $\delta p(z)=\delta n(z)$, the depth $z$ extending from the beam entry surface at $z=0$ into the sample 48, 49. Extending the surface-less solution $\delta p=G \tau$ (Eq. 2.20) to a depth-dependent trial solution

$$
\delta p(z)=G \tau+A \exp \left(-\frac{z}{L}\right)
$$

and choosing the boundary condition

$$
\left.D \frac{\mathrm{d} \delta p}{\mathrm{~d} z}\right|_{z=0}=s \cdot \delta p(z=0)
$$

leads to a solution which can be written as

$$
\delta p=G \tau_{e f f}
$$


with an effective lifetime $\tau_{\text {eff }}$ and may be converted to a squared effective diffusion length

$$
L_{e f f}^{2}(z)=L^{2}\left(1-\frac{\frac{s}{D} L}{1+\frac{s}{D} L} \exp \left(-\frac{z}{L}\right)\right)
$$

with the minority charge carrier diffusion coefficient $D$. This equation emphasizes the depth dependence of the diffusion limitation and the profound influence of the surface recombination velocity for this geometry. If the sample has the shape of a lamella, where two surfaces are present and their distance (sample thickness $t$ ) is comparable to the diffusion length, the approach may be modified by choosing $z=0$ in the center of the sample, using the boundary conditions

$$
\begin{aligned}
-\left.D \frac{\mathrm{d} \delta p}{\mathrm{~d} z}\right|_{z=t / 2} & =s \cdot \delta p\left(z=\frac{t}{2}\right) \\
\left.D \frac{\mathrm{d} \delta p}{\mathrm{~d} z}\right|_{z=-t / 2} & =s \cdot \delta p\left(z=-\frac{t}{2}\right)
\end{aligned}
$$

and extending the trial solution to

$$
\delta p(z)=G \tau+A\left(\exp \left(\frac{z}{L}\right)+\exp \left(-\frac{z}{L}\right)\right) .
$$

The depth-dependent effective diffusion length then becomes

$$
L_{e f f}(z)=L \sqrt{1+\frac{s \cdot L}{D} \frac{\exp \left(-\frac{z}{L}\right)+\exp \left(-\frac{z}{L}\right)}{\left(1-\frac{s \cdot L}{D}\right) \exp \left(-\frac{t}{2 L}\right)-\left(1+\frac{s \cdot L}{D}\right) \exp \left(\frac{t}{2 L}\right)}} .
$$

Integrating and averaging $\delta p(z)$ over the whole sample thickness enables extraction of the sample thickness dependent effective diffusion length

$$
L_{e f f}(t)=\int_{z=-t / 2}^{t / 2} \frac{L_{e f f}(z)}{t} \mathrm{~d} z=L \sqrt{1-\frac{L}{\frac{t}{2} \cdot \frac{D}{s \cdot L}+\operatorname{coth}\left(\frac{t}{2 L}\right)}} .
$$

Still, an exact analytical solution to the diffusion equation (Eq. 2.38) for two surfaces cannot be given. Nevertheless, Tan et al. used a method of a large number of image charges to formulate an approximate numerical solution, which, however, demands for a large computational effort [50]. It should be noted that this treatment is valid only in the low injection regime, where injection and EBIC signal increase linearly with the generation, i.e. the beam current [51]. If high injection conditions apply, the interpretation of EBIC signal is significantly complicated 47,52 .

\subsubsection{Heterojunctions}

So far, only so-called p-n homojunctions have been covered by the introduced model, i.e. $\mathrm{p}$-n junctions consisting of $\mathrm{p}$ - and $\mathrm{n}$-doped regions of the same material, e.g. silicon. However, if the material of the p-doped region is different from the n-doped region, a so-called p-n heterojunction forms. A common example is a GaAs-Ge junction.

In the formation of a heterojunction, the same processes as in a homojunction occur, i.e. the equilibrium of drift and diffusion currents and the adjust of a common Fermi energy level $E_{F}$. Yet, both materials of a heterojunction generally exhibit different bandgaps $E_{G}$ and electron affinities $\chi$. Equation 2.28 shows that the difference in $\chi$ can also be 


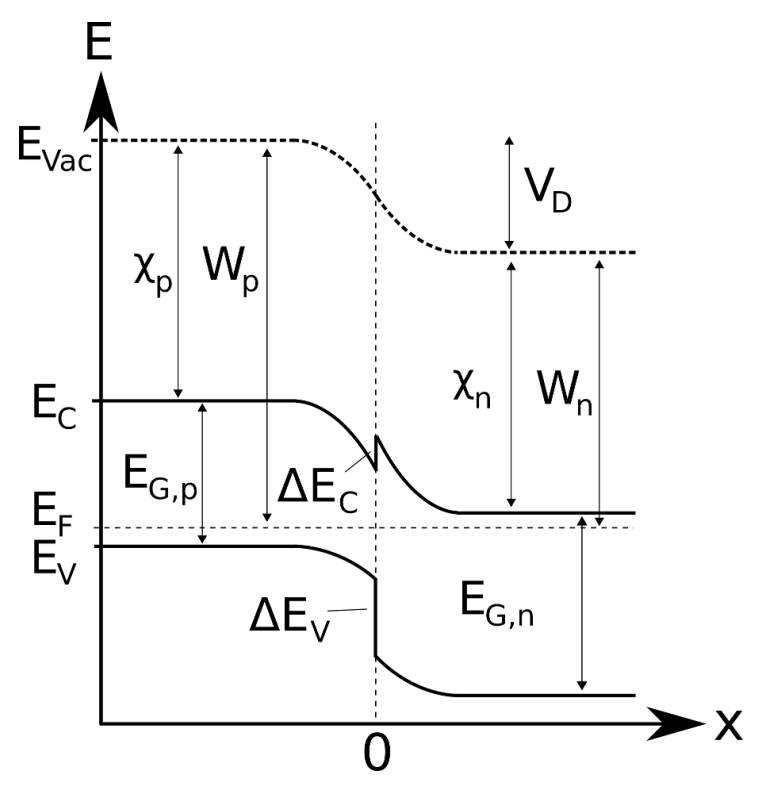

Figure 2.6: Sketched band diagram of a p-n heterojunction in equilibrium. Compared to the homojunction (Fig. 2.1), the electron affinity $\chi$ and band gap $E_{G}$ are different on both sides of the junction. This leads to the band offsets $\Delta E_{C}$ and $\Delta E_{V}$, while the vacuum energy $E_{v a c}$ is only dependent on the work function $W$ and does not exhibit offsets.

expressed by different work functions $W$, which shall prove more convenient later.

A model for band diagrams of p-n heterojunctions was developed by Anderson [53]. It shows that at the p-n junction interface $(x=0)$, band discontinuities arise because of the different electron affinities. They are shown in Fig. 2.6 and can be described by the band offsets 32

$$
\Delta E_{C}=\chi_{p}-\chi_{n} \text { and } \Delta E_{V}=E_{G, n}-E_{G, p}-\Delta E_{C} .
$$

For use in a heterojunction model, equations $2.2,2.5$ and 2.29 have to be modified in order to take into account changing parameters from the p-doped to the n-doped region, thus incorporate them as step functions. These parameters are: permittivity $\varepsilon$, work function $W$, electron and hole mobilities $\mu_{p}$ and $\mu_{n}$, electron and hole diffusion coefficients $D_{p}$ and $D_{n}$ and intrinsic charge carrier concentration $n_{i}$. To take these into account, it is instructive to use the general forms of the equations, using the (polarisation-less) electric displacement field $\vec{D}=\varepsilon \vec{E}$ and the Quasi Fermi levels for holes and electrons, $E_{F, p}$ and $E_{F, n}$, respectively. If the simple Einstein relations (equation 2.7) are assumed valid, equations $2.2,2.5$ and 2.29 can thus be written as 16

$$
\begin{aligned}
\vec{J}_{p} & =\mu_{p} p \cdot \vec{\nabla} E_{F, p} \\
\vec{J}_{n} & =\mu_{n} n \cdot \vec{\nabla} E_{F, n} \\
\vec{\nabla} \cdot \vec{D} & =\rho .
\end{aligned}
$$

Furthermore, we can transform equations 2.12 and 2.13 so that they refer to the electron and hole concentrations $p_{0}(x)$ and $n_{0}(x)$ in the neutral region. These are material dependent constants which can be defined as step functions, as explained below. The change in potential over the sample as well as the step-like change of the bulk work function $W(x)$ 
(which does not include the potential bending) have to be taken into account to define $p$ and $n$ correctly at any position $x$. Introducing the Quasi Fermi levels, and staying within the Boltzmann approximation, equations 2.12 and 2.13 can be rearranged to 54

$$
\begin{aligned}
& E_{F, p}=E_{V}-k_{B} T \cdot \ln \left(\frac{p}{N_{V}}\right)=-e\left(V-V_{0}\right)-\chi-E_{G}-k_{B} T \cdot \ln \left(\frac{p}{N_{V}}\right) \\
& E_{F, n}=E_{C}+k_{B} T \cdot \ln \left(\frac{n}{N_{C}}\right)=-e\left(V-V_{0}\right)-\chi+k_{B} T \cdot \ln \left(\frac{n}{N_{C}}\right)
\end{aligned}
$$

with the potential reference $V_{0}$ (see also Fig. 2.6). Introducing the bulk work function with the help of the bulk concentration step functions $p_{0}(x)$ and $n_{0}(x)$,

$$
W=\chi+E_{g}+k_{B} T \cdot \ln \left(\frac{p_{0}}{N_{V}}\right)=\chi-k_{B} T \cdot \ln \left(\frac{p_{0}}{N_{C}}\right),
$$

enables transformation of Eq. 2.49 and 2.50 to

$$
\begin{aligned}
& E_{F, p}=-e\left(V-V_{0}\right)-W-k_{B} T \cdot \ln \left(\frac{p}{p_{0}}\right) \\
& E_{F, n}=-e\left(V-V_{0}\right)-W+k_{B} T \cdot \ln \left(\frac{n}{n_{0}}\right)
\end{aligned}
$$

and thus

$$
\begin{aligned}
& p=p_{0} \exp \left(\frac{-e\left(V-V_{0}\right)-W-E_{F, p}}{k_{B} T}\right) \\
& n=n_{0} \exp \left(\frac{e\left(V-V_{0}\right)+W+E_{F, n}}{k_{B} T}\right) .
\end{aligned}
$$

It should be noted that, using only $V, W, p_{0}(x)$ and $n_{0}(x)$, the band offsets $\Delta E_{C}$ and $\Delta E_{V}$ of the heterojunction do not have to be taken into account explicitly. The hole and electron concentrations in the neutral region $p_{0}$ and $n_{0}$ as well as the work function $W$ are position dependent in a heterojunction and given as step functions along the x-direction. The majority carrier concentrations $p_{p, 0}$ and $n_{n, 0}$ in the neutral region are, in the case of doping (much higher than $n_{i}$ ), approximately equal to the doping concentrations, whereas the minority charge carrier concentrations $p_{n, 0}$ and $n_{p, 0}$ are obtained from the former using the local law of mass action (equation 2.23):

$$
\begin{aligned}
& p_{0}(x) \approx \begin{cases}N_{A}, & x<0 \text { ( } \mathrm{p} \text {-doped region }) \\
\frac{n_{i, n}^{2}}{N_{D}}, & x \geq 0 \text { (n-doped region })\end{cases} \\
& n_{0}(x) \approx \begin{cases}\frac{n_{i, p}^{2}}{N_{A}}, & x<0 \text { ( } 1 \text {-doped region }) \\
N_{D}, & x \geq 0 \text { (n-doped region) }\end{cases}
\end{aligned}
$$

Equations 2.53 and 2.54 can be further simplified by choosing the constants wisely. As we use the common equilibrium Fermi level as energy reference in our system (see Fig. 2.6), and no bias voltage is applied, we can set the quasi Fermi levels as zero: $E_{F, p}=E_{F, n}=0$. Furthermore, we fix $\mathrm{p}$ and $\mathrm{n}$ to the equilibrium neutral region values at the sample edges (here described as $\pm \infty): p(x=-\infty)=p_{p, 0}, n(x=\infty)=n_{n, 0}$. By fixing the potential $V(x=-\infty) \equiv 0$, we obtain the reference potential $V_{0}$ and the diffusion voltage $V_{D}$ as defined in equation 2.27 .

$$
V_{0}=\frac{W_{p}}{e} \Longrightarrow V(x=\infty)=\frac{W_{p}-W_{n}}{e}=V_{D}
$$


Thus $V(x=\infty)$ becomes equal to the diffusion voltage. Finally, we obtain from equations 2.53 and 2.54

$$
\begin{aligned}
& p=p_{0} \exp \left(\frac{-e V-\left(W-W_{p}\right)}{k_{B} T}\right) \\
& n=n_{0} \exp \left(\frac{e V+\left(W-W_{p}\right)}{k_{B} T}\right) .
\end{aligned}
$$

Due to the definitions of $p_{0}, n_{0}$ and $W$, we may introduce a further simplification for this choice of reference potential. Defining $p_{0, W}:=p_{0} \exp \left(\frac{W-W_{p}}{k_{B} T}\right)$ and $n_{0, W}:=$ $n_{0} \exp \left(\frac{W-W_{p}}{k_{B} T}\right)$, thus

$$
\begin{aligned}
p_{0, W}(x) & \approx \begin{cases}N_{A}, & x<0 \text { (p-doped region }) \\
\frac{n_{i, n}^{2}}{N_{D}} \exp \left(\frac{e V_{D}}{k_{B} T}\right), & x \geq 0 \text { (n-doped region) }\end{cases} \\
n_{0, W}(x) & \approx \begin{cases}\frac{n_{i, p}^{2}}{N_{A}}, & x<0 \text { (p-doped region) } \\
N_{D} \exp \left(\frac{-e V_{D}}{k_{B} T}\right), & x \geq 0 \text { (n-doped region) }\end{cases}
\end{aligned}
$$

Eq. 2.58 and 2.59 simplify to

$$
\begin{aligned}
& p=p_{0, W} \exp \left(\frac{-e V}{k_{B} T}\right) \\
& n=n_{0, W} \exp \left(\frac{e V}{k_{B} T}\right) .
\end{aligned}
$$

This definition has the advantage that in the case of homojunctions, $p_{0, W}$ and $n_{0, W}$ become constants for the whole junction.

The Quasi Fermi levels (Eq. 2.51 and 2.52 also simplify using $p_{0, W}$ and $n_{0, W}$. Using them in the current equations 2.46 and 2.47 and reintroducing the diffusion coefficients $D_{p}(x)$ and $D_{n}(x)$ via the Einstein relations (equation 2.7) leads to the current densities modified for heterojunctions

$$
\begin{aligned}
\frac{1}{e} \vec{J}_{p} & =p \cdot\left(-\mu_{p} \cdot \vec{\nabla} V+\frac{D_{p}}{p_{0, W}} \vec{\nabla} p_{0, W}\right)-D_{p} \vec{\nabla} p \\
-\frac{1}{e} \vec{J}_{n} & =n \cdot\left(\mu_{n} \cdot \vec{\nabla} V+\frac{D_{n}}{n_{0, W}} \vec{\nabla} n_{0, W}\right)-D_{n} \vec{\nabla} n .
\end{aligned}
$$

The general Poisson equation 2.48 can be written similarly to equation 2.29, but taking the position dependent $\varepsilon(x)$ into account:

$$
\vec{\nabla} \cdot \vec{D}=-\vec{\nabla} \cdot(\varepsilon \vec{E})=e \cdot\left(p-n+C_{i o n}\right)
$$

The space charge region widths as obtained from the box approximation for homojunctions (Eq. 2.31 and 2.32) also have to be altered for heterojunctions [53]:

$$
\begin{aligned}
d_{p} & =\sqrt{\frac{2 \varepsilon_{p} V_{D}}{e} \frac{\varepsilon_{n} N_{D}}{N_{A}\left(\varepsilon_{p} N_{A}+\varepsilon_{n} N_{D}\right)}} \\
d_{n} & =\sqrt{\frac{2 \varepsilon_{n} V_{D}}{e} \frac{\varepsilon_{p} N_{A}}{N_{D}\left(\varepsilon_{p} N_{A}+\varepsilon_{n} N_{D}\right)}}
\end{aligned}
$$




\section{$2.2 \mathrm{PCMO}$ and STNO}

This section is about the general features of perovskites, going further into detail for STNO and PCMO and concluding in properties of devices composed of these materials.

\subsubsection{Perovskite structure}

The perovskite structure was first discovered in the material $\mathrm{CaTiO}_{3}$, where the crystal structure also takes its name from. The general chemical composition of this material class is $A B C_{3}$. In inorganic perovskite materials, $A$ is usually a rare earth or an alkali metal, $B$ a transition metal and $C$ an oxygen or halogen ion. In contrast, in metal-organic perovskites the $A$ site is filled by organic molecules [55]. Ideally, the atomic structure is cubic, with the $A$ ion taking the primitive lattice sites, the $B$ ion the body centered sites and the $C$ ion the face centered sites, as shown in Fig. 2.7.

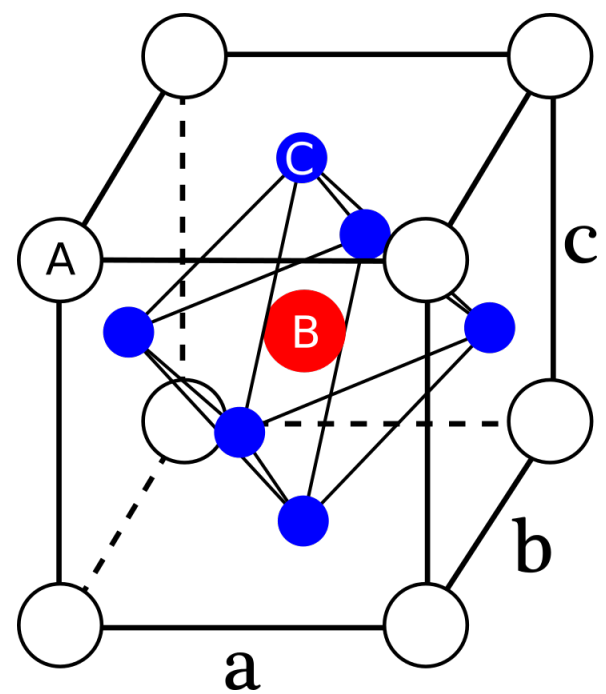

Figure 2.7: Sketch of the ideal perovskite $A B C_{3}$ unit cell. The $A$ ion takes the primitive, the $B$ ion the body centered and the $C$ ion the face centered sites. The unit cell lengths $a$, $b$ and $c$ are equal. The thin lines highlight the $B C_{6}$ octahedron, which is important to characterize manganites.

However, perovskite materials do not necessarily exhibit a perfectly cubic crystal structure. The real structure heavily depends on the actual ions on the lattice sites. The deviation from the ideal cubic structure can be expressed using the ratio of the mean atomic radii $\left\langle r_{A}\right\rangle,\left\langle r_{B}\right\rangle$ and $\left\langle r_{C}\right\rangle$, in the Goldschmidt tolerance factor

$$
\Gamma=\frac{1}{\sqrt{2}} \frac{\left\langle r_{A}\right\rangle+\left\langle r_{C}\right\rangle}{\left\langle r_{B}\right\rangle+\left\langle r_{C}\right\rangle}
$$

which was defined in [56]. The radii are averaged over all respective sites, as the population of a lattice site is not generally spatially homogeneous in doped materials. In a close-packing model, the interatomic distances between species $i$ and $j$ are $r_{i}+r_{j}$. Thus, in the ideal cubic structure, $\left\langle r_{A}\right\rangle+\left\langle r_{C}\right\rangle=\sqrt{2}\left(\left\langle r_{B}\right\rangle+\left\langle r_{C}\right\rangle\right)$, leading to $\Gamma=1$. In this case, the structure is free of stress. If $\Gamma$ is different from 1 in none-ideal perovskite structures, the structure changes into a rhombohedric (for $0.96<\Gamma<1$ ) or orthorhombic $(\Gamma<0.96)$ shape [57]. In these cases, the different ionic radii of the different species lead 
to mechanical stress, which may result in the occurrence of dislocations. These structural changes lead to a variety of changing properties. Only taking the changes of the lattice as a whole into account, magnetic phase transitions of perovskite materials can be explained [58]. However, other features common to perovskite structure materials require more advanced effects to be fully explained, as will be demonstrated in the section about PCMO.

\subsubsection{Polarons}

Charge carriers in materials with strong correlations can often be described by the polaron quasiparticle concept, which was developed by Landau and Pekar [59. It is particularly useful in transition metal oxides [60], which also includes perovskite materials. A polaron describes the coupling of an electron (or other mobile charge carrier, e.g. protons [61]) with a lattice distortion in a polarizable medium. Due to Coulomb interaction, an electron may deform the lattice of a crystalline material it is travelling through. The distortion will travel with the electron, thus they can be described by a single quasiparticle.

Depending on the strength of coupling of the electron to phonons (another quasiparticle concept used to describe lattice vibrations), the nature of polarons can be dramatically different. Different cases were defined from the Holstein-Hamilton operator [62 of a polaron as electron-induced phonon and are classified as follows [63]. A polaron can be adiabatic or anti-adiabatic, weak or strong, and small or large. If the motion of the polaron is determined by the lattice ions, i.e. the electrons follow them, a polaron is adiabatic. In the vice-versa case, it is anti-adiabatic. If an electron is bound to a single phonon, it is classified as a weak polaron, if it interacts with more than one phonon, it is a strong polaron. Finally, if the polaron is only weakly localised, i.e. influences many different lattice sites, it is classified as a large polaron, while if it is localised to neighboring ions, it is a small polaron. Particularly, a small strong polaron and a large weak polaron are viewed as the two defining extremes. They are shown in Fig. 2.8(a) and (b).

An imminent consequence of the polaron nature is found in the temperature behaviour of the polaron mobility in a lattice, as shown in Fig. 2.8(c). The mobility of small polarons can be described by thermally activated hopping for high temperatures and by tunneling between lattice sites for low temperatures [64, 65], while large polarons can be understood as free charge carriers, scattered at lattice vibrations [66. This results in opposing trends of the mobility behaviour with temperature of both polaron types.

\subsubsection{Niobium doped strontium titanate}

A standard example for an inorganic perovskite is strontium titanate (STO) in its undoped form $\left(\mathrm{SrTiO}_{3}\right)$, which exhibits a nearly perfectly cubic perovskite structure (Goldschmidt tolerance factor $\Gamma=1.001$ ). An alternate form is the Nb-doped STNO $\left(\mathrm{SrTi}_{1-y} \mathrm{Nb}_{y} \mathrm{O}_{3}\right)$. The crystal structure is virtually insensitive to doping at the $\mathrm{B}$ site up until a doping fraction of $y=0.02$, with no change in $\Gamma$. While STO is paramagnetically insulating at room temperature [69,70], it becomes increasingly conductive with increased doping, showing a metallic temperature behaviour of resistivity [71], but overall similar features to an electron-doped semiconductor [72]. In this work, the doping fraction $y=0.002$ is used. STNO exhibits a large bandgap at room temperature of $E_{g}=3.2 \mathrm{eV}$ 73. This doping ratio corresponds to a doping concentration of $N_{D}=3 \cdot 10^{19} \mathrm{~cm}^{-3}$. 

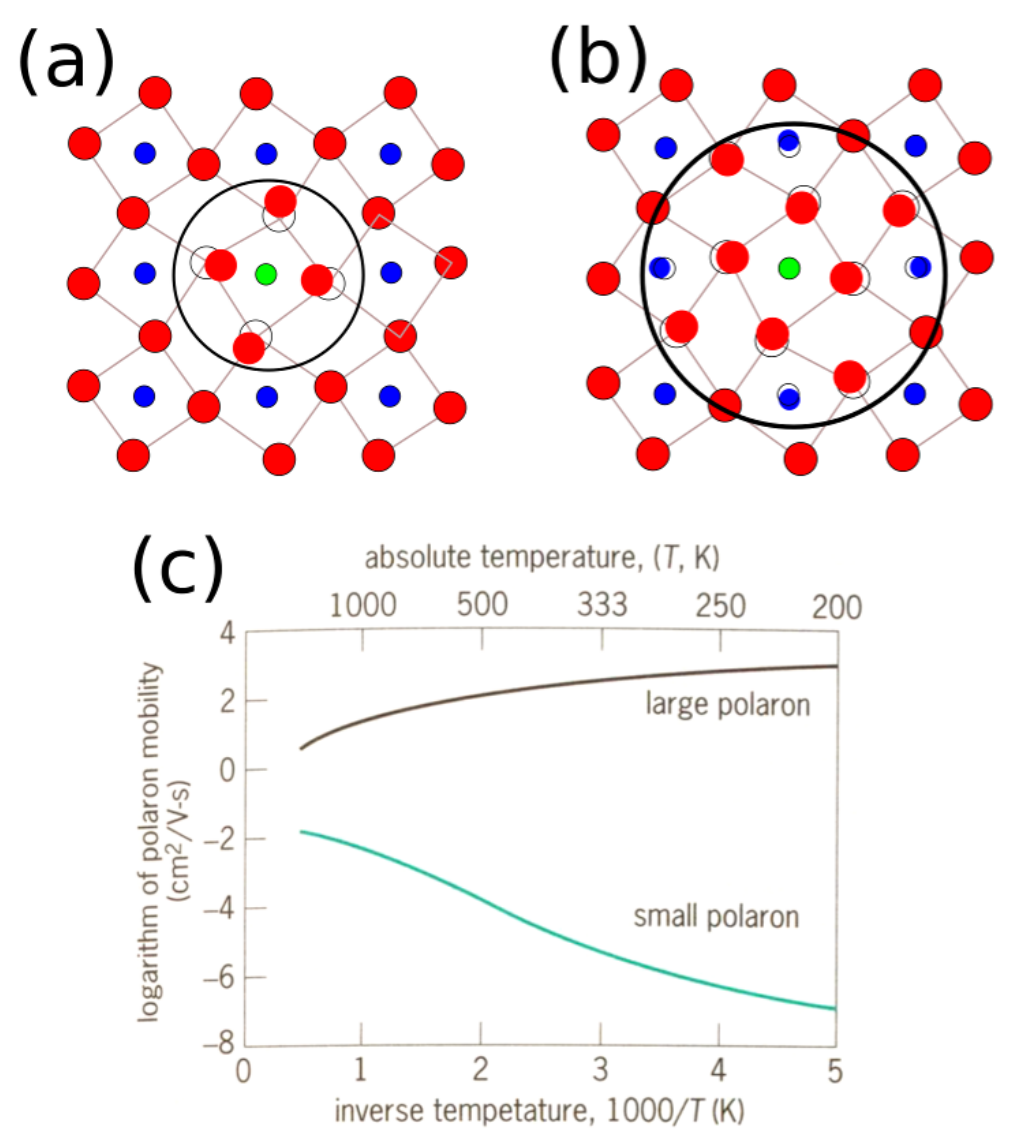

Figure 2.8: Illustration of the extreme cases of a (a) strong, small and a (b) weak, large polaron, modeled after 67]. (c) shows the opposing mobility behaviour of these two polaron types with temperature 68

Even though polaronic charge carriers exist in STNO, their nature is that of a weak polaron due to weak electron-phonon coupling, in a mixture of small and large polarons 72 , 74, 75]. The mobility of the polaronic charge carriers at room temperature was estimated from resistivity measurements to be around $\mu=1 \frac{\mathrm{cm}^{2}}{\mathrm{~s}} \mid 76$.

\subsubsection{Calcium doped praseodymium manganite}

In contrast to strontium titanate, manganites feature a plethora of effects which can be traced back to their deviation from the ideal cubic perovskite structure under various external influences. A thorough compendium of the effects described in the following is available by Dagotto [5]. In this work, the emphasis is on calcium doped praseodymium manganite PCMO $\left(\operatorname{Pr}_{1-x} \mathrm{Ca}_{x} \mathrm{MnO}_{3}\right)$. Due to the similar size of $\mathrm{Pr}$ and Ca atoms, the tolerance factor does not change dramatically with doping. For $x=0.36$, the Goldschmidt tolerance factor is $\Gamma=0.957$ [77], leading to a noticeable deviation from the ideal cubic perovskite structure. It is convenient to describe this effect in terms of the $\mathrm{MnO}_{6}$ octahedra, which are visible in the ideal perovskite unit cell (Fig. 2.7) as the octahedron formed by the oxygen (site C) ions surrounding the manganese (site B) ion. Compared to their ideal position, the reduced Goldschmidt factor leads to a tilting of the $\mathrm{MnO}_{6}$ octahedra against each other, as illustrated in Fig. 2.9, in order to compensate 
for the different interionic distances $\mathrm{Pr} / \mathrm{Ca}-\mathrm{O}$ and $\mathrm{Mn}-\mathrm{O}$. This is a cooperative effect between neighboring unit cells, ideally extending over the whole perovskite body. In this case, the unit cell has to be viewed differently: The a and b axis are no longer oriented as indicated in Fig. 2.7, but are rotated by 45 degrees, as shown by the dotted lines in Fig. 2.9(a). Furthermore, the c axis is doubled, as shown by the arrows in Fig. 2.9(b). The lattice constants in this unit cell model are $a=0.5418(1) \mathrm{nm}, b=0.5457(3) \mathrm{nm}$ and $c=0.7669(1) \mathrm{nm} 78$. In this work, a slightly lower doping $x=0.34$ is used, corresponding to a high hole doping concentration of $N_{A}=6.3 \cdot 10^{21} \mathrm{~cm}^{-3}$.
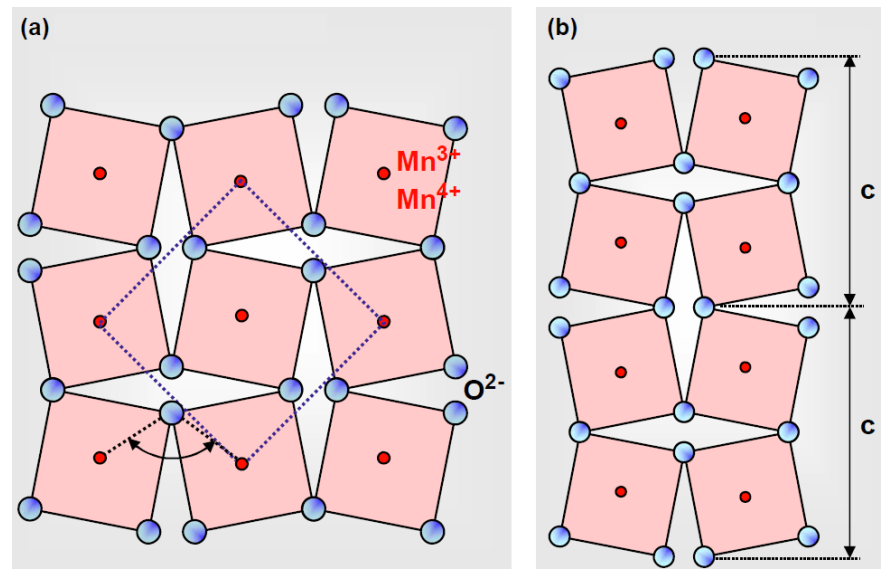

Figure 2.9: Sketch of the octahedral tilt in planes (a) parallel to a and b axis and (b) parallel to the $\mathrm{c}$ axis. This tilt occurs in case of a Goldschmidt factor $\Gamma<1$ and leads to an effective change of the lattice constants, as indicated by the dotted lines. A site ions are not shown. Modified from 79 .

By controlling whether manganites form paramagnetic insulating (PMI) or ferromagnetic metallic (FMM) phases, the $\mathrm{MnO}_{6}$ octahedral tilt has direct consequences to the electronic and magnetic configuration of manganites [58]. The atomic orbitals of A site atoms $\mathrm{Pr}$ or Ca do not significantly overlap with the $\mathrm{Mn}$ and $\mathrm{O}$ ions, but the orbitals of the latter strongly overlap, making the $\mathrm{MnO}_{6}$ octahedra especially important. The electronic and magnetic configuration of the material is dominated by the $\mathrm{Mn}$ ion, which changes its valency depending on its surrounding, whereas $\mathrm{O}$ is mostly insensitive to this due to it being close to noble gas configuration. The Mn 3d orbitals, which are degenerate for the free $\mathrm{Mn}$ ion, are energetically split in the crystal field of a $\mathrm{MnO}_{6}$ octahedron into two $e_{g}$ orbitals with higher energy and three $t_{2 g}$ orbitals with lower energy than the degenerate states (see Fig. 2.10 (a)). This is essentially a result of the different orbital shapes, which overlap with the oxygen $2 p$ orbitals.

Aside from the crystal field splitting of orbitals, another possible reduction of degeneracy arises due to the Jahn Teller effect [80]. This effect sets in if one of the $e_{g}$ orbitals is occupied, leading to $\mathrm{MnO}_{6}$ octahedrals compressing in two axis and expanding in the third one (see Fig. 2.10 (b)). The direct result of this distortion is another energetic split of $3 d$ orbitals, i.e. the two $e_{g}$ and two of the three $t_{2 g}$ orbitals split (see Fig. 2.10 (a)). The local lattice distortions controlled by an electron may be described as polarons and move through the lattice. The predominant polaron type in manganites is that of a strong, small polaron due to strong electron-phonon coupling [81]. The mobility of these charge carriers at room temperature is relatively low, with a lower limit of $\mu=0.02 \frac{\mathrm{cm}^{2}}{\mathrm{~s}}$, as estimated from resistivity measurements of PCMO films [76. 

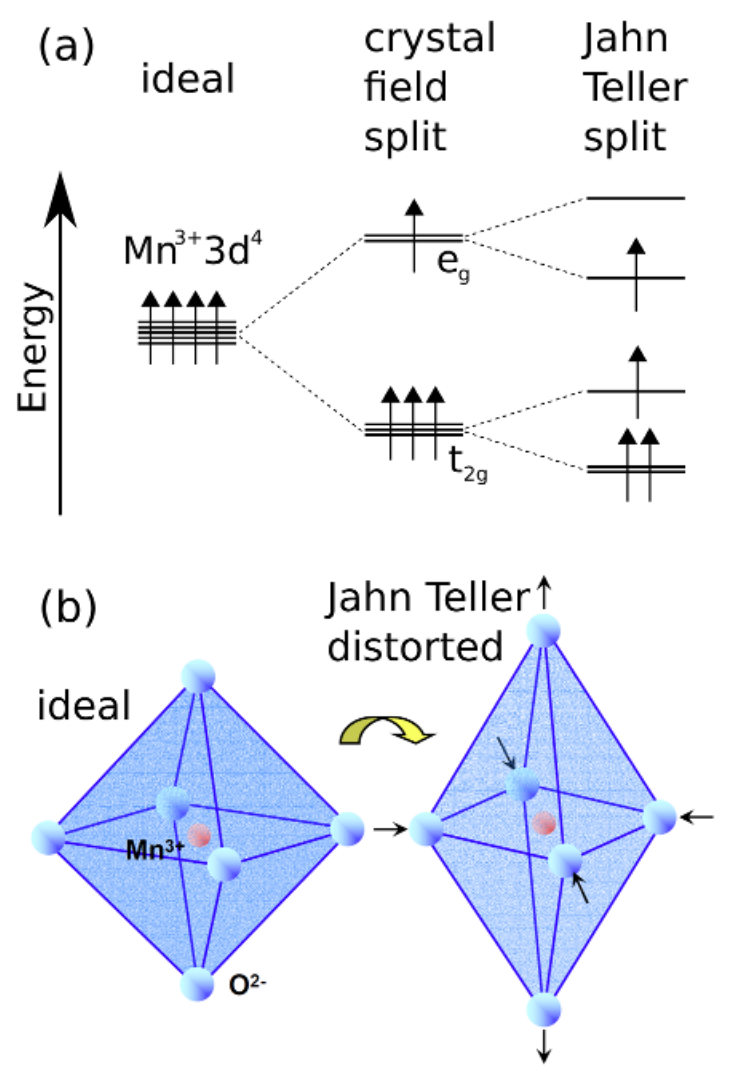

Figure 2.10: Splitting of $\mathrm{MnO}_{6}$ 3d orbitals. (a) Splitting of energy levels due to crystal field splitting and the Jahn Teller distortion, after [13]. The degenerate band from the ideal case split into $e_{g}$ and $t_{2 g}$ bands, which are further split due to the Jahn Teller effect. In the case of $\mathrm{Mn}^{4+}, e_{g}$ orbitals are unoccupied, preventing the Jahn Teller split. (b) Illustration of the Jahn Teller distortion of a $\mathrm{Mn}_{6}$ octahedral- Modified from 79

Optical illumination can excite electrons in manganite material from one state to another. In PCMO with $x=0.34$, there are two predominant optical excitations possible [14]. One is the so-called charge transfer $(\mathrm{CT})$ transition, in which charge is moved from the oxygen to the manganese ion. The other is the so-called Jahn Teller (JT) excitation, happening on the Mn site. The transitions can be explained in the density of states (DOS) diagram shown in Fig. 2.11, which was developed from a density functional theory $\mathrm{MnO}_{6}$ dimer [13, 82. The dimer is also called a Zener polaron. The JT transition is metastable in a cooperative state with other dimers, which is responsible for polaronic excitations found in PCMO with a lifetime on the order of $1 \mathrm{~ns}$ at low temperatures [83]. Another, weaker excitation at energies below $1 \mathrm{eV}$ is due to photon-assisted polaron hopping [15.

By tuning the Ca doping ratio $x$ from 0 to 1 , the nominal valency of the Mn ions can be changed from +3 to +4 . For in-between ratios, this results in a statistical distribution of $\mathrm{Mn}^{3+}$ and $\mathrm{Mn}^{4+}$ ions at room temperature. The influence on $\mathrm{Mn}$ valency is, in fact, the predominant influence of the $\mathrm{Pr} / \mathrm{Ca}$ ions on the $\mathrm{MnO}_{6}$ octahedrals and on the electronic and magnetic structure of PCMO as a whole. The Jahn Teller effect only happens in $\mathrm{MnO}_{6}$ octahedra with $\mathrm{Mn}^{3+}$ ions. Furthermore, the doping ratio also controls at which temperature phase transitions occur in PCMO. At low temperatures, PCMO transitions from a paramagnetic insulated [84, unordered state at room temperature into a charge 


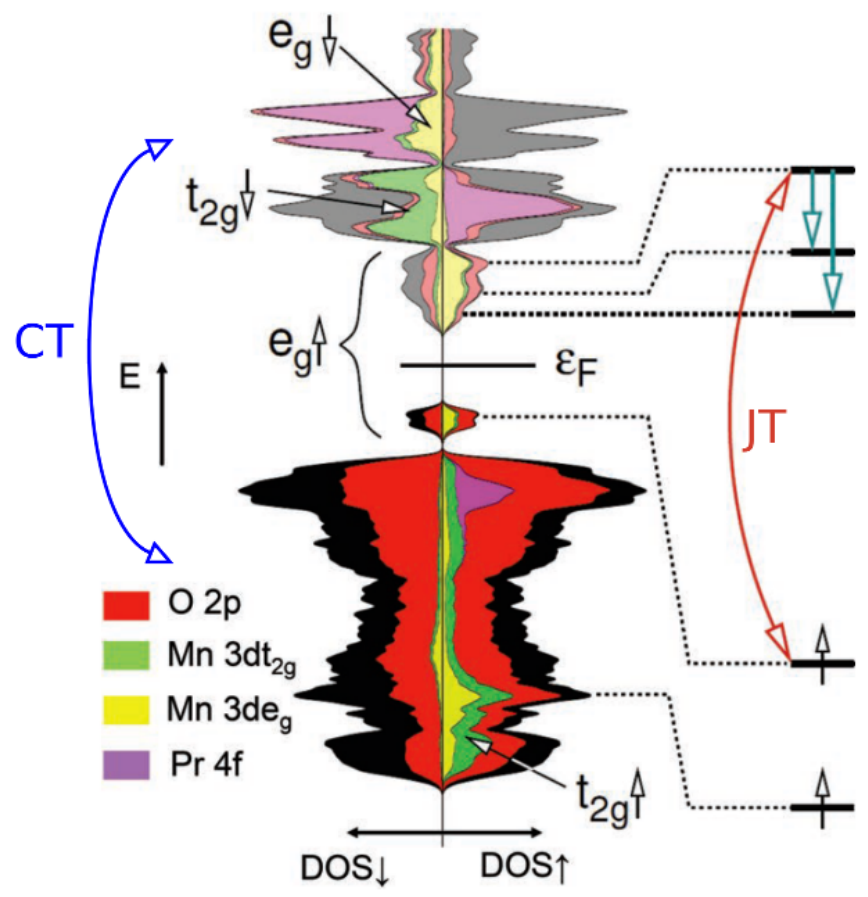

Figure 2.11: Density of states diagram of a PCMO $(x=0.35)$ dimer, modified from 13]. Charge transfer (CT) and Jahn Teller (JT) transitions are indicated. The latter leads to a metastable state, where an electron is trapped in the third $e_{g}$ state from the top for a timespan of about $1 \mathrm{~ns}$ before relaxing to the lowest $e_{g}$ state.

ordered state. This is a first order phase transition, i.e. it happens in a broad temperature range and domain-wise. The charge ordered phase enables cooperative effects between the $\mathrm{MnO}_{6}$ octahedra which are responsible for the metastable $e_{g}$ state mentioned before. For even lower temperatures, the system enters into a phase with antiferromagnetic (AFM) order. The phase transitions happen around characteristic temperature $T_{C O}$ and the Neel temperature $T_{N}$. These temperatures vary with doping, as can be seen in the phase diagram in Fig. 2.12

Using external excitations by electric or magnetic fields, PCMO may also enter into states of particularly low resistance at low temperatures, which is possible due to strong correlation effects of the polaronic charge carriers. The first example is the colossal electro-resistance, setting in at the temperature region around $T_{C O}$ and under the application of strong electric fields, but also depends on the electrical pre-history [85 87]. The low resistance regime is connected to the "melting" of the charge ordered phase due to the strong electric field. The effect was also shown in p-n junctions [9, 12]. The second effect is the colossal magneto-resistance at low temperatures and in strong magnetic fields, which is common for many manganites [57, 88] and was shown at metal-PCMO interfaces [89]. Thus, it can be concluded that the occurrence of Jahn Teller distortions, including its interplay with octahedral tilt, as well as the formation of several electric and magnetic states can be controlled in PCMO by changing the Ca doping level. 


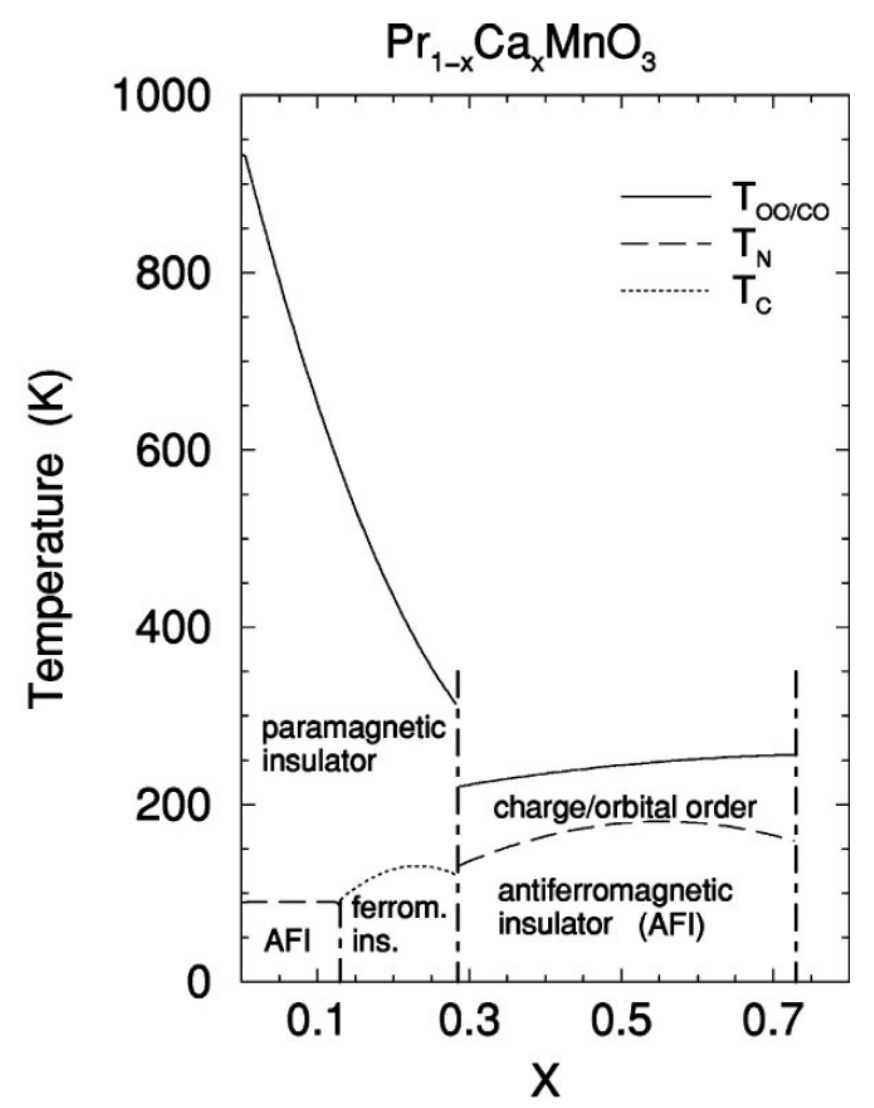

Figure 2.12: Phase diagram of PCMO for varying Ca doping fraction $x$, taken from 10 . In the different phases, electrical and magnetic behaviour changes dramatically, and long range ordering is present in the charge/orbital ordered phase.

\subsubsection{PCMO-STNO p-n junctions}

P-doped PCMO layers grown on top of $n$-doped STNO substrate have been found to form p-n junctions with clearly rectifying behaviour for high-quality epitaxially grown films [11. In Fig. 2.13, exemplary $I(U)$ characteristics from polychromatic illumination are shown. The spectrally resolved absorptance of both materials at room temperature is compared in Fig. 2.13. (a). Overall, the broadband absorptance of PCMO from near infrared to near ultraviolet light is contrasted with semiconductor-like STNO absorptance peaking at an interband transition at the bandgap energy $E_{g}=3.2 \mathrm{eV}$. The other peaks are identified as follows [14. The weak STNO peak at around $2.4 \mathrm{eV}$ is usually related to a transition to mid-gap states, which are regarded as originating from oxygen vacancies. The absorptance increase below $2 \mathrm{eV}$ is identified with intra-band quasiparticle excitations, which relax almost immediately and can thus not be collected by the p-n junction. In PCMO, two features dominate the absorptance spectrum, which have been described before, both extending across several $\mathrm{eV}$. The charge transfer transition sets in around $2.5 \mathrm{eV}$, and the Jahn Teller transition is centered at $1.6 \mathrm{eV}$. For comparison, the spectrum of near-CMO $(x=0.95)$ is also shown, where the absorptance due to the Jahn Teller transition is absent as the Jahn Teller effect is not present there.

The one diode Shockley model (equation 2.37) has been applied to PCMO-STNO $I(U)$ characteristics, allowing to extract the short circuit current density $J_{s c}$, open circuit 

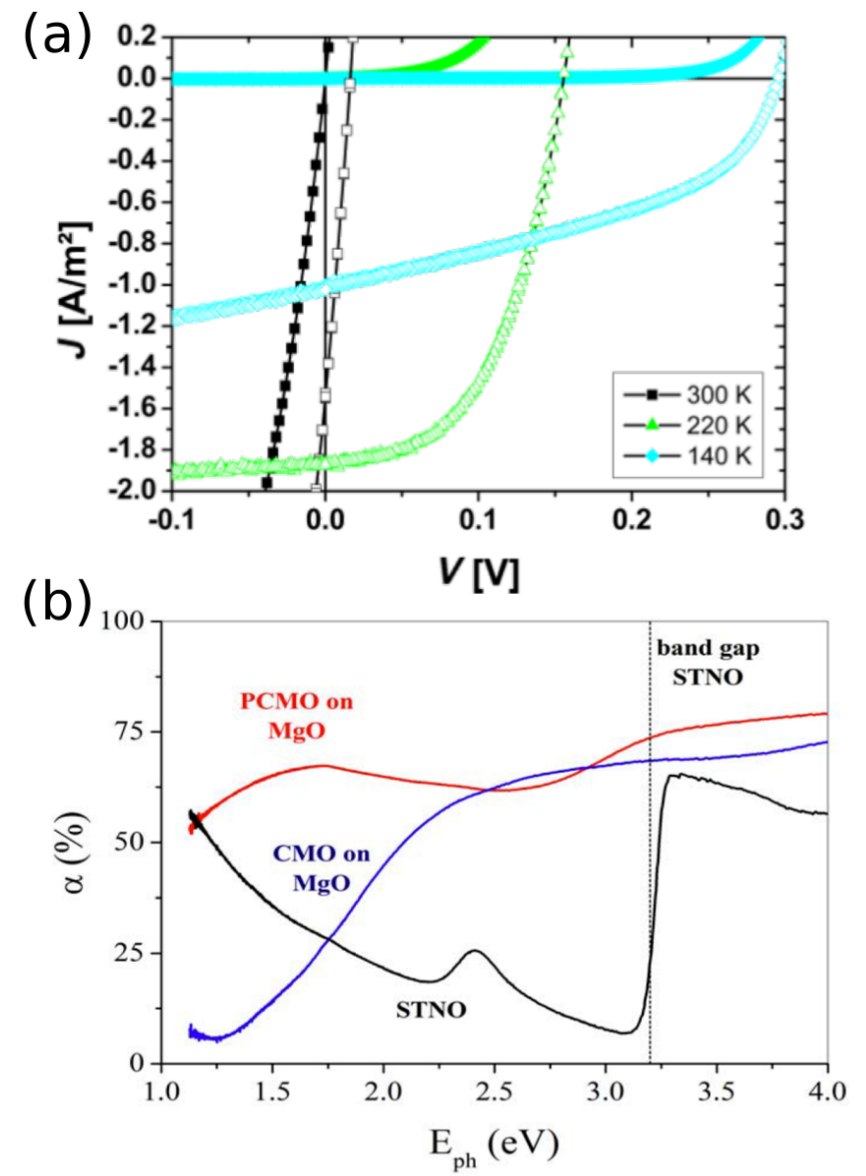

Figure 2.13: General features of PCMO-STNO p-n junctions. (a) $I(U)$ characteristics of a nonannealed sample for polychromatic illumination, taken from 12. (b) Spectrally resolved absorptance of STNO and PCMO, with CMO as comparison with absent Jahn Teller effect, taken from [14]. The identified transitions are discussed in the text.

voltage $V_{o c}$, as well as parallel and serial resistance $R_{p}$ and $R_{s}$. The temperature characteristics of these materials provide measures of the overall junction quality, e.g. they are sensitive to improved ordering in the charge ordered phase of post-growth annealed PCMO. Furthermore, they allow extracting the energy barrier of thermally activated transport and provide a fingerprint for the CER effect [12, 14].

The behaviour of thermally activated transport can for example be seen by the decreasing short circuit current with decreasing temperature, and the increased charge separating behaviour of the junction in the charge ordered phase is seen by the increasing open circuit voltage (see Fig. 2.14(a)). Illuminating the junction with spectrally filtered light further allows isolating the contribution of the two dominant PCMO transitions. By plotting $V_{o c}\left(J_{s c}\right)$ for different temperatures (Fig. 2.14(b)), the different slopes in different temperature ranges even reveal the characteristic behaviour in the different phases as shown in the PCMO phase diagram (Fig. 2.12 ). It can be deduced that while at room temperature, the dominant part of collected excess charge carriers is generated directly at the PCMO-STNO interface, in the charge ordered phase the dominant part is generated in the bulk and is thus subject to diffusion with a diffusion length larger than the space charge region size. In the antiferromagnetic phase, the low polaron mobility is 
the dominant limitation of charge carrier collection.
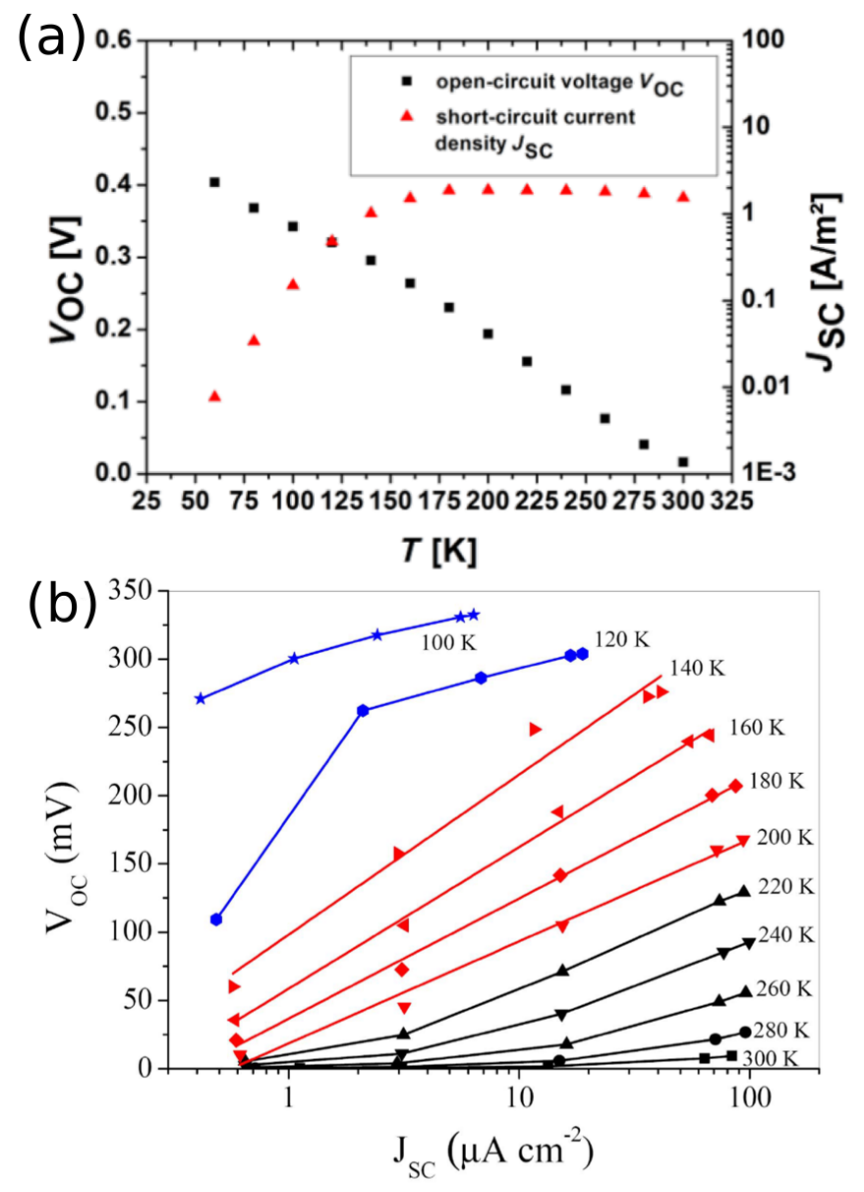

Figure 2.14: Temperature behaviour of short circuit current density $J_{s c}$ and open circuit voltage $V_{o c}$ in non-annealed PCMO-STNO p-n junctions. (a) Comparing temperature trends of a sample under polychromatic illumination, taken from [12. $I_{s c}(T)$ is clearly dominated by thermally activated hopping, while $V_{o c}(T)$ shows the improving charge separation with decreasing temperature. (b) Plotting $V_{o c}\left(I_{s c}\right)$ for different temperatures using data from different spectral filters allows access to the different PCMO phases, namely the antiferromagnetic phase (blue data), the charge ordered phase (red data) and the non-ordered room temperature phase (black data). Taken from $[12$.

\subsection{Electron microscopy}

In this work, high energy electron beams are provided by electron microscopes. Electron microscopy has a long tradition since Ruska's and Knoll's first idea of electron lenses in 1932 (reviewed in [90]) and has advanced significantly in the last 20-30 years due to implementation of stable high tension sources and electronics, effective aberration correctors and various analytic methods to be used within the microscopes. The physical principles of the methods used in this work are explained in the following. A compendium, especially for transmission electron microscopy, was written by Williams and Carter [91]. 


\subsubsection{Scanning electron microscopy}

The electron beam in all microscopes used in this work use Schottky emitters as their electron source. Electrons emerging from a heated tungsten tip with tip size on the atomic level are extracted by a low extraction voltage and accelerated by a strong electric field, providing the acceleration voltage, in an evacuated column. The used acceleration voltages range from just a few hundred $\mathrm{eV}$ in scanning electron microscopes for especially sensitive samples to several hundred $\mathrm{keV}$ common for transmission electron microscopes. Compared to more traditional electron sources such as $\mathrm{LaB}_{6}$ cathodes, the source size is significantly smaller at Schottky emitters, providing a higher possible resolution, and also the energetic spread of the electrons is on the order of below $1 \mathrm{eV}$.

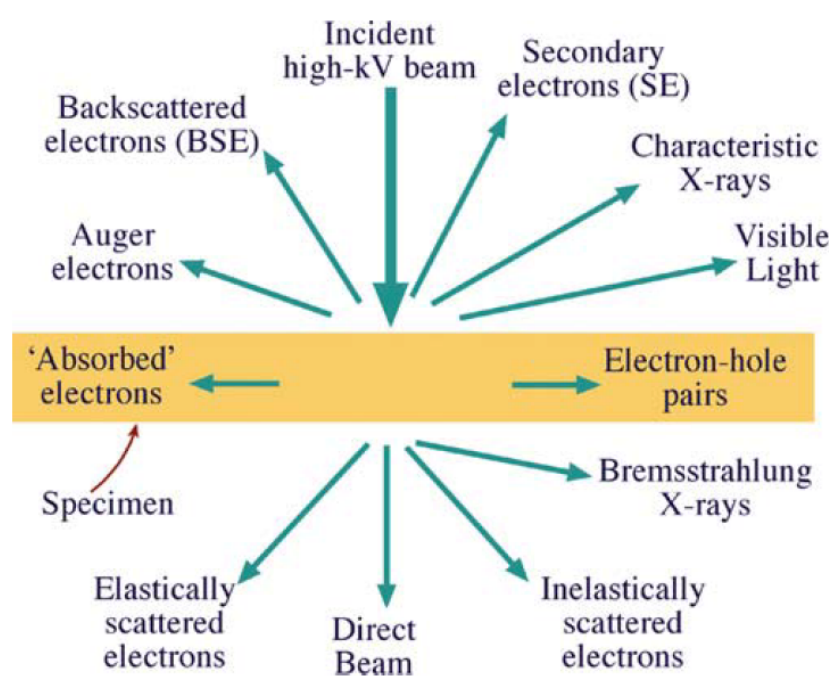

Figure 2.15: Interactions of an impinging electron beam with a sample, taken from 91 . The direct beam and the elastically and inelastically scattered electrons are only present in TEMs, not in SEMs. Electron hole pairs can be examined by EBIC.

The accelerated electrons are focused in a system of electromagnetic lenses, called condensor system, before reaching the sample, forming a converging beam and providing a probe size on the sample surface in the nanometer range. The sample chamber is evacuated as well, in order to provide undisturbed passage of the electrons before hitting the sample. In the beam's interaction with the sample, secondary (SE) as well as backscattered electrons (BSE) are emitted (see Fig. 2.15 for an illustration of all relevant emissions). While the SE provide a mostly topological contrast, while BSE are sensitive to chemical differences [92]. Both can be collected by an Everhart-Thornley detector, which is essentially a combination of a scintillator and a photomultiplier. However, it has to be taken into account that BSE usually have higher energy than SE and are concentrated on smaller reflection angles with respect to the impinging beam. Furthermore, $\mathrm{x}$-rays are produced as impinging electrons may also kick out electrons from the inner shells of sample atoms, leading to transitions of electrons from outer shells under emission of x-ray photons. These also provide chemical contrast and may be collected by special $\mathrm{x}$-ray detectors.

The beam is scanned in two dimensions in order to form an image using coils in the column containing the lens system, and the beam position extracted from the voltages on the scanning coils synchronized to the signal on the chosen detector. In order to obtain 
the optimum image resolution, the sample has to be brought into the height where the beam has its lowest diameter, which is called working distance. The most important lens error affecting SEM images is usually astigmatism, resulting from asymmetries in lens polepieces. It may be corrected independently in both image axis by two stigmator lenses.

\subsection{2 (Scanning) transmission electron microscopy}

The main difference from transmission electron microscopes (TEM) to scanning electron microscopes (SEM) is the position and size of the sample. Whereas in SEM the sample is usually thick enough to absorb the electron beam completely, in the TEM the electron beam is transmitted by the sample, which usually has a thickness on the order of $1 \mu \mathrm{m}$ or less. In the sample, the beam is subject to elastic and inelastic scattering, resulting in forward scattering and phase change of the incoming beam. Just as in SEM, it is also possible to use detectors to record various emitted signal (e.g. energy dispersive x-ray EDX - for chemical analysis).

In conventional TEM, the condensor system is used to form a broad parallel beam. A typical TEM ray diagram is illustrated in Fig. 2.16. It is possible to record a magnified real image of the sample, or a diffraction pattern. Apertures may be used to filter out samples regions (selected area diffraction - SAD) or diffraction spots (objective aperture). The scattering potential provides access to the sample's microstructure, and the projection direction of the potential can be controlled by tilting the sample with respect to the electron beam.

As an alternative, many TEMs may also be used in scanning mode, called STEM. Here the electron beam is focused by the condensor system to a convergent beam with a diameter of less than $1 \mathrm{~nm}$. This beam only interacts in a small volume of the sample. The focused beam is set to form a diffraction pattern on the screen or the CCD camera, and the scattered electrons are collected by annular detectors (see Fig. 2.17). The beam can be scanned by coils in the column to provide an image of a larger sample area. As such, this mode is comparable to SEM, but uses different signals to form the image. Detectors with different radii are used, which are mainly discriminated into bright field, annular dark field, and high angle annular dark field detectors. The actual scattering angles collected by them can be adjusted by the projection system, controlling the camera length. The advantage of STEM compared to TEM is the immediate access to different diffraction contrasts without using apertures, as well as the possibility to record various detector signals in a spatially resolved way.

Aside from astigmatism, which may be corrected in the same way as in SEM, the dominant lens error affecting image resolution in (S)TEM usually is spherical aberration. This error arises from different scattering angle of electron passing electron lenses off the optical axis, compared to on-axis electrons, leading to points being imaged as disks of finite size. In the TEM case the objective lens, the strongest lens in the system, is predominantly responsible for aberration. It is possible to correct this error by inserting an additional lens setup between the objective lens and the projector system, which is called aberration corrector [93, 94]. This setup is rather complex, involving several entangled magnetic fields with a complex geometry. The same setup can be used to correct the spherical aberration of the condensor system, however in that case the corrector must be placed in this lens system. Usually TEMs only have one kind of aberration corrector installed. 
(A)

(B)

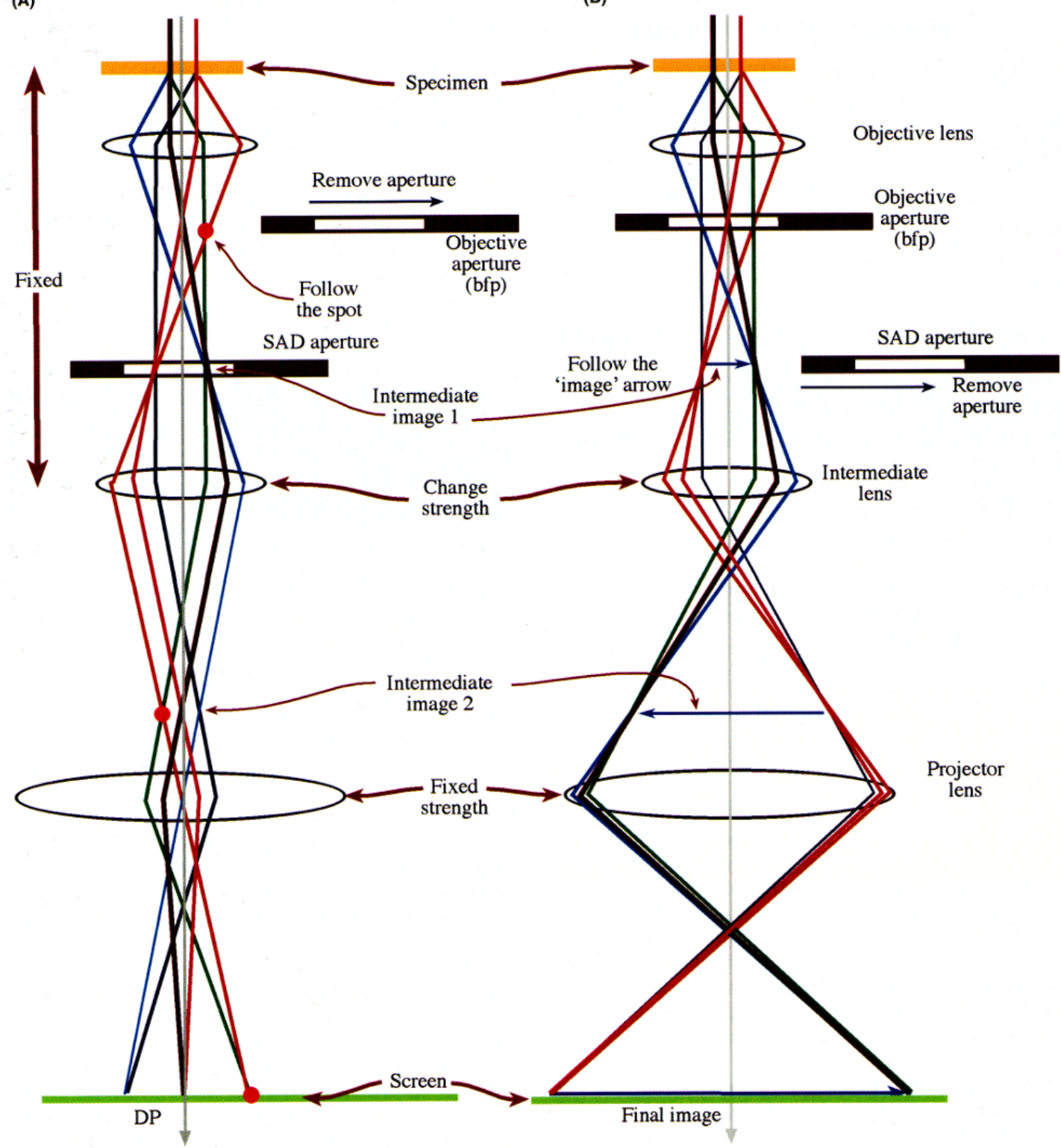

Figure 2.16: Simplified TEM ray diagram, taken from 91 . A broad electron beam impinges the specimen. Depending on the setting of the intermediate lens, a diffraction pattern (DP, image $(\mathrm{A})$ ) or real image $(\mathrm{B})$ can be recorded. The projector lens magnifies the DP or image. Using apertures, a part of the sample (selected area diffraction $\mathrm{SAD}$ ) or single diffraction spots (objective aperture) can be isolated.

\subsubsection{Electron energy loss spectroscopy}

Electron energy loss spectroscopy (EELS) is a method to analyze the chemical constituents of a sample as well as their bonding states. It is based on the discrimination of single electrons from the beam in their energy after passing through the sample, which is realized by a spectrometer. Spectra for each point in a STEM map may be collected, which may be processed into energy-filtered profiles and images. The energy distribution of the initial beam is ideally Gaussian shaped around the center energy defined by the acceleration voltage, with the distribution width defining the EELS setup's energy reso- 


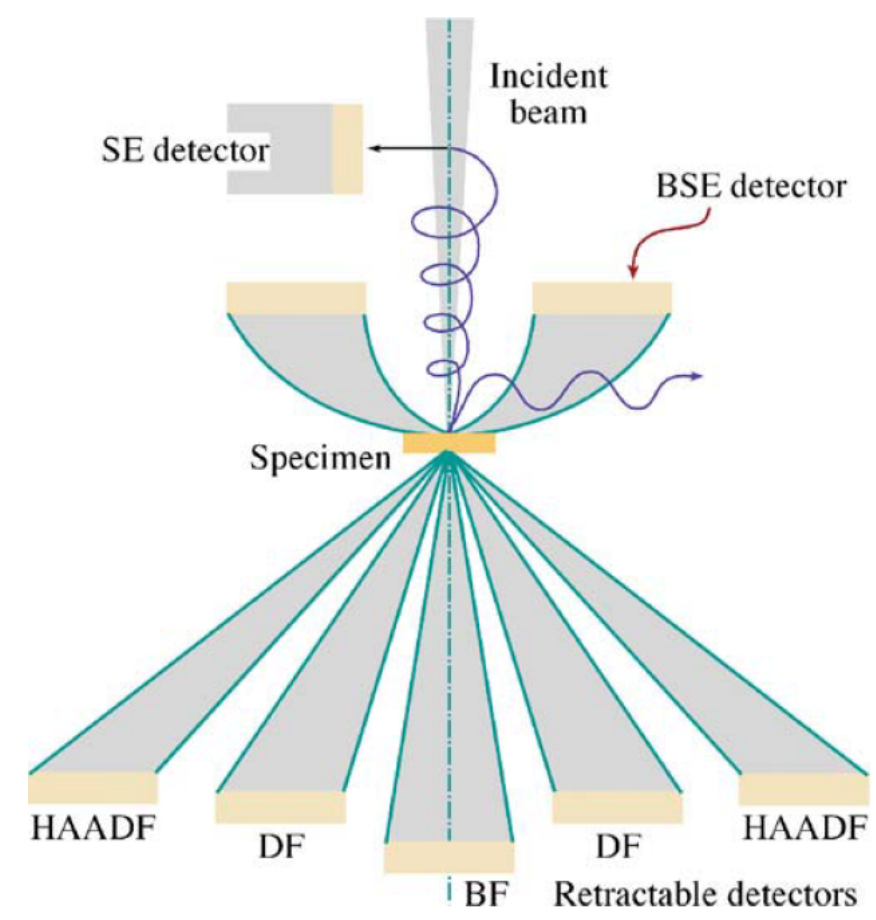

Figure 2.17: Principle STEM setup, with focused electron beam impinging the sample and STEM detectors for collecting scattered electrons from different angles, taken from 91. In most TEMs, only bright field (BF), annular dark field (DF) and high angle annular dark field (HAADF) detectors are available.

lution. The mean initial electron energy is assigned as zero energy loss, while electrons leaving the sample with less energy have a positive loss. A negative loss is also possible for electrons with more than the mean energy.

Features in the energy loss spectrum additional to the zero loss peak (ZLP), as exemplified in Fig. 2.18 (a), can be split into four categories: (1) very low loss excitations (also called valence band EELS - VEELS), including band-band excitations, phonon losses, surface plasmons and relativistic losses (Cherenkov radiation) 95, (2) plasmonic excitations, which are a series of Lorentzian shaped peaks resulting from collective excitations of electrons in the conduction band (for a semiconductor) with a characteristic frequency, the number of peaks depending on sample thickness; (3) elemental absorption edges, which are specific to certain transitions in the core shell of atoms, similar to EDX lines; and (4) electron loss near-edge fine structure (ELNES), the fine structure in elemental edges which can be connected to the chemical state of the respective atom, i.e. its bonding state in the sample structure. The spectrum is generally divided into a very low loss $(<5 \mathrm{eV})$, a low loss (about $5-50 \mathrm{eV})$ and a core loss region $(>50 \mathrm{eV})$. The very low loss region is usually dominated by the tail of the zero loss peak, unless a beam monochromator is used to improve the energy resolution to better than $1 \mathrm{eV}$. In this case, the aforementioned processes come into play. In the low loss region, the plasmon peaks are dominant, with few elemental edges present, while the core loss region is dominated by core loss features superimposed with a background defined by the plasmon peaks. Comparing the integrated energy loss intensity for the zero loss peak $I_{0}$ and the rest of the spectrum $I_{t}$, the sample thickness $t$ in units of the electron inelastic mean free path 
$M F P$ can be extracted:

$$
\frac{t}{M F P}=\ln \left(\frac{I_{t}}{I_{0}}\right)
$$

Because of the mathematical form of Eq. 2.70, this is called the log-ratio method.

Two different EELS setups are common, the in-column and the bottom-mounted setup. The latter is the relevant setup here, where, in principle, after passing through a variable entrance aperture to cut off elastically scattered electrons, the beam is energy-split in a prism optics, realigned in a projector lens system and collected on an additional CCD camera. See Fig. 2.18 for an illustration. A compendium about the EELS method was written by Egerton [96], also touching on many aspects of EFTEM, which is described in the following.
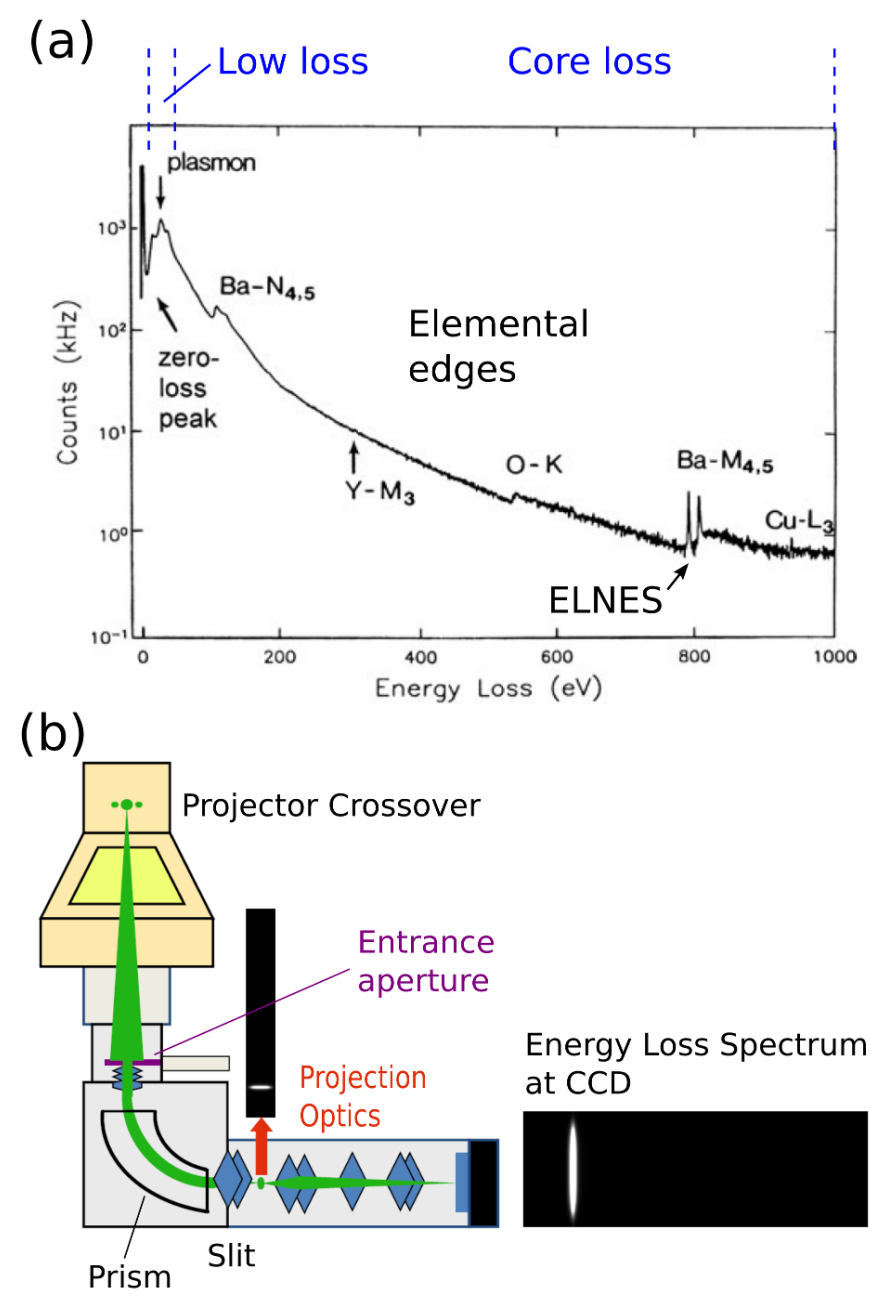

Figure 2.18: (a) EELS spectrum example from $\mathrm{YBa}_{2} \mathrm{Cu}_{3} \mathrm{O}_{7}$ in logarithmic scale with the different typical regions and features marked. Modified from [96]. (b) Spectrometer optics ray diagram for a Gatan GIF Quantum 965. Modified from 97.

\subsubsection{Energy filtered transmission electron microscopy}

Using the EELS spectrometer optics with a conventional (broad) TEM beam, another method of obtaining energy filtered images may be used, called energy filtered TEM 
(EFTEM). A slit in the setup can be used to filter arbitrary parts of the energy spectrogram. The optics may be aligned to form an image instead of a spectrogram on the CCD camera, which is just composed of the filtered electrons. Various information may be highlighted in the filtered images, for example the sample thickness may be extracted in a similar way to the log-ratio method. For this two-window method, an unfiltered image and an image filtered for energies around the zero loss peak are compared according to Eq. 2.70. While being a faster method than EELS mapping to obtain energy filtered images when starting from conventional TEM imaging, EELS mapping is generally more flexible in the energy-dependent processing (e.g. background subtraction) and allows for higher energy resolution.

\subsubsection{Off-axis electron holography}

In principle, electron holography is used to gain access to the phase of the object wave forming a (conventional) TEM image, which is lost in the recording process as explained before. While many different methods are available, in this work the off-axis holography method is used [98]. It uses a Möllenstedt biprism, which is implemented as a goldcovered wire under variable bias voltage inserted near the objective lens back focal plane 99, 100. This setup enables a coherent superposition of an unperturbed reference wave with the sample-dependent object wave. The interference pattern, which is the actual hologram, contains both amplitude and phase of the object wave [101]. They can be separated by Fourier analysis by isolating the center band and one of the side bands with numerical apertures. The method has been successfully applied to measure the built-in electric field of p-n junctions 102, 103] as well as displacement fields in atomic resolution [104 and also profits from numerical correction of residual lens errors [105].

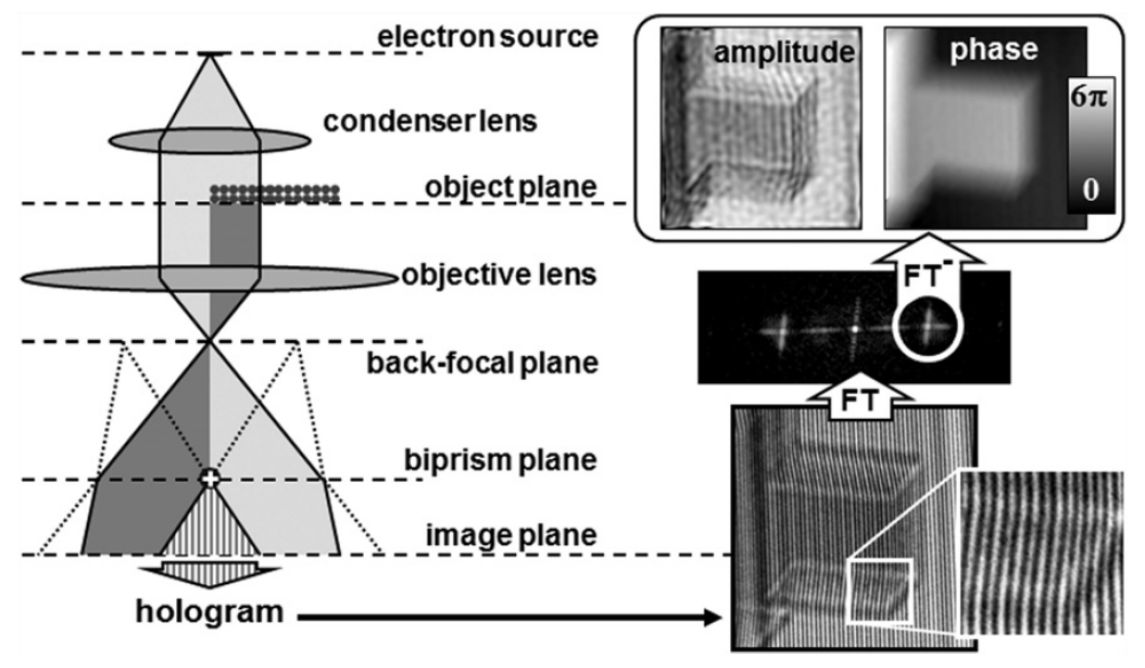

Figure 2.19: Principle of off-axis electron holography, taken from [104]. On the left side, the interference of an electron beam passing the sample object (object wave) with the unperturbed initial beam (empty wave) due to the biprism is sketched, leadings to the hologram formation. The right hand sides shows, from bottom to top, the extraction of the object wave amplitude and phase from the hologram via Fourier transform filtering. The example shows images of an $\mathrm{MgO}$ crystal. 


\subsection{Simulation}

Simulations are used in this work as a means of deepening the understanding of experimental results. Two softwares are used, CASINO (monte CArlo SImulation of electroN trajectory in sOlids) and COMSOL Multiphysics, which feature Monte Carlo and finite element algorithms, respectively. They are described briefly in the following. The exact simulation conditions are described together with the simulation results, starting in $\mathrm{Ch}$. 4.

\subsubsection{Monte Carlo simulation in CASINO}

The program CASINO is used to simulate the electron beam generation volume in samples of arbitrary chemical composition. It is based on code by Hovington et al. [106 and was extended in its version 2 [107] by a graphical user interface with the ability to combine several sample layers and to calculate the three-dimensional distribution of dissipated energy in the sample within an automatically chosen volume. However, this version is limited to acceleration energies below $30 \mathrm{keV}$. Version 3 is optimized to also use energies of several hundred $\mathrm{keV}$, includes an option to define the simulated volume within a sample of given size, and options for many different two or three-dimensional sample shapes as well as presets for special applications [108].

The operating principle is explained in the following. First, the sample may be defined. For each layer, an average mass density is used, which is calculated from the given chemical composition. The initial electron energy, the beam size, its impingement point on the sample and its rotation angle to the sample surface normal can also be chosen. The finite beam size is implemented as a two-dimensional Gaussian distribution around the given impingement point with the given size as $99.9 \%$ of all impinging electrons. The principle simulation process is repeated for a given number of electrons in order to provide a statistical distribution of dissipated energy. The energy loss is calculated with a Monte Carlo algorithm via a series of elastic scattering events in the sample. The collision angle of an event, changing the electron path, is calculated from a set of given partial elastic Mott cross-sections and a random number [109]. The distance between two scattering events is calculated from another random number with a logarithmic probability distribution, giving the fraction of the elastic mean free path of the given material travelled between the events [109]. The elastic mean free path is calculated taking into account the density distribution of an amorphous material with the given chemical composition and the single elements' total elastic scattering cross sections. Instead of inelastic scattering events, a continuous energy loss between the elastic scattering events is assumed, with the energy change along the electron path being calculated from a modified Bethe's stopping power equation given by Joy and Luo [110, 111]. The process is terminated if the simulated electron reaches an energy below $50 \mathrm{eV}$ or leaves the sample at the defined surfaces. The dissipated energy along its path is registered in a three-dimensional discrete mesh in the sample defined by the user.

\subsubsection{Finite element simulation}

Finite element simulations of EBIC measurements are performed in the program COMSOL Multiphysics. The basic mathematical principle is that partial differential equations, such as the Poisson equation and the charge carrier equations defined in equations 2.6 . 


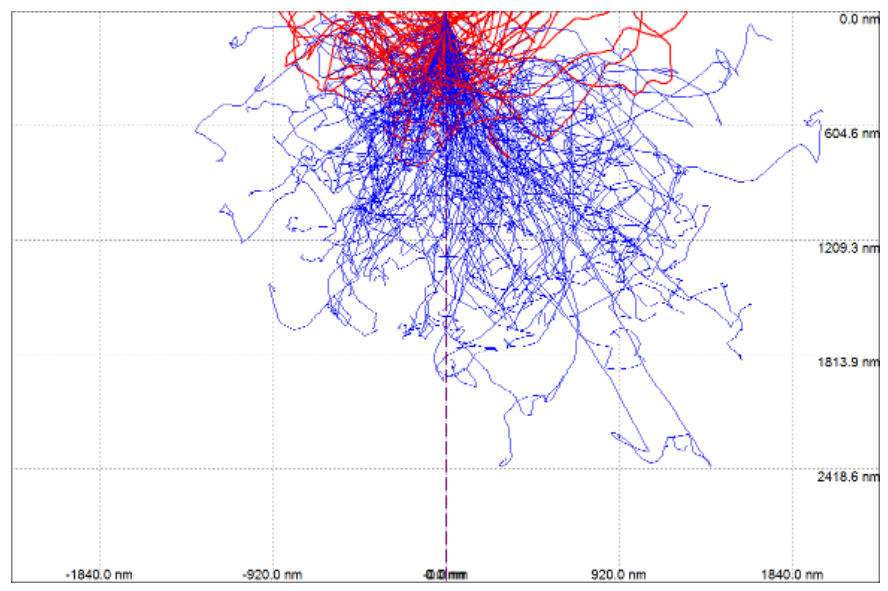

Figure 2.20: CASINO v2 Monte Carlo simulation of $15 \mathrm{kV}$ electron paths in a PCMO-STNO sample, with the excitation happening at the layer interface. $2 \cdot 10^{5}$ electrons were simulated. Backscattered electrons, which leave the sample at the beam entry surface, are highlighted in red. The asymmetry of the left and the right hand side is due to the two materials' different mass density.

2.2 and 2.3, are defined within a certain one, two, or three dimensional sample volume with given Dirichlet or Neumann boundary conditions [112]. To solve this problem numerically, it is convenient to reformulate this problem into a discrete set of equations, which can also be written as a matrix eigenvalue problem. The approximate numerical solution is best if the matrix is as sparse as possible. To obtain this, the base functions of the eigenvectors have to be defined in sub-volumes of the sample volume which are as small as possible. These sample volumes form the mesh. In two dimensions, it is composed of triangles and in one dimension, of line segments. Each sample volume is assigned a coordinate. If just these coordinates are considered instead of the continuous sample volume, the problem reduces to a linear set of equations. This problem can be solved directly, e.g. with Gauss elimination, or iteratively, e.g. with the Gauss-Seidel method. In the end, the solution for each coordinate is linearly interpolated over the whole subvolume, taking into account the nearest neighbor coordinates. In practice, the mesh size has to be chosen small enough that the solution is as exact as desired, but large enough to ensure reasonable computation time.

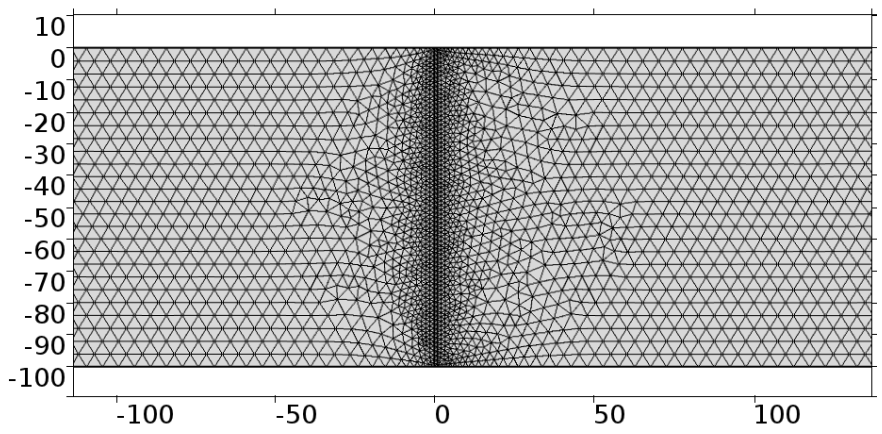

Figure 2.21: Example of a COMSOL mesh, with triangle elements. An adaptive mesh refinement leads to finer meshing at the interface in the center. Coordinates are in nanometers. 


\section{Chapter 3}

\section{Experimental setup and methods}

In this chapter the sample preparation, experimental setup and the used experimental methods are described. Furthermore, the processing of the experimental data are detailed.

\subsection{Sample preparation}

The preparation of the samples used for EBIC and holography experiments in this work are briefly described in the following.

\subsubsection{PCMO-STNO samples}

The PCMO-STNO samples were grown by Benedikt Ifland using reactive ion beam sputtering of PCMO on a commercial STNO substrate. The single crystalline substrates are composed of $\mathrm{SrTiO}_{3}$ with $0.1 \mathrm{wt} \% \mathrm{Nb}$ doping, equating to a doping level of $y=0.002$ in $\mathrm{SrTi}_{1-y} \mathrm{Nb}_{y} \mathrm{O}_{3}$. The PCMO sputter target was backed out of a mixture of $\mathrm{Pr}_{6} \mathrm{O}_{11}$, $\mathrm{CaCO}_{3}$ and $\mathrm{Mn}_{2} \mathrm{O}_{3}$ powder, which were combined in specific ratios to receive the desired target composition, which is $\operatorname{Pr}_{1-x} \mathrm{Ca}_{x} \mathrm{MnO}_{3}$ with $x=0.34$ in this work. The carbon vanishes from the powder during the baking process.

The sputtering method uses a Kaufmann source and Xe as the sputter gas, which is inserted into the source at a pressure of $1 \cdot 10^{-4} \mathrm{mbar}[77]$. A $20 \mathrm{~mA}$ current of Xe ions, which have been accelerated with $1 \mathrm{kV}$ and neutralized after acceleration, hits the sputter target, knocking out atoms from the target which are accelerated towards the substrate afterwards. The target angle to the substrate is aligned to allow stoichiometric deposition. The substrate is heated to $750^{\circ} \mathrm{C}$ during the sputtering process in order to ensure the deposited atoms can move enough to allow homogeneous layer growth. Still, island growth is prevalent during the layer growth [113]. The sputtering process is conducted in an oxygen atmosphere with oxygen partial pressure of $1.4 \cdot 10^{-4}$ mbar in order to compensate for oxygen deficiency in the layer.

After the growth, the quality of the PCMO layer was checked by x-ray diffractometry, confirming a single-crystalline layer grown epitaxially on the substrate. PCMO grows in [010] in-plane and [001] out-of-plane direction on an STNO substrate oriented in [001] in-plane and [001] out of plane direction. Due to considerable stress in the grown PCMO layer, $\mathrm{MnO}_{z}$ precipitates tend to form on the layer surface [113].

The metallic contact layers are grown in the same sputtering device onto the sample surface in vacuum and heating the substrate to $200^{\circ} \mathrm{C}$. Au is used as PCMO (front side) contact while Ti, followed by a protective Au layer is used as STNO (back side) contact. Both form Ohmic contacts to the respective materials. 
Two forms of sample geometries are used in this work, the structured and the unstructured geometry. In the unstructured geometry, both the PCMO and the front side contact $\mathrm{Au}$ layer are grown over the whole substrate area (see Fig. (a)). The layer thicknesses are $t_{\mathrm{PCMO}}=370 \mathrm{~nm}$ and $t_{\mathrm{Au}} \approx 800 \mathrm{~nm}$ in this case. In the structured geometry, shadow masks are used to shield a part from the substrate from layer growth. PCMO is grown only in the center of the STNO substrate, and two contact layers of lateral size $1.4 \mathrm{~mm}^{2}$ are deposited on PCMO and STNO, respectively, so that front side and back side contacts are both located on the sample surface (see Fig. (b)). The layer thicknesses in this case are $t_{\mathrm{PCMO}}=100 \mathrm{~nm}, t_{\mathrm{Au}} \approx 300 \mathrm{~nm}$ and $t_{\mathrm{Ti}} \approx 50 \mathrm{~nm}$. The back side contact of the unstructured sample is later deposited on a slice of the sample used for lamella preparation, see Sec. 3.8.3.
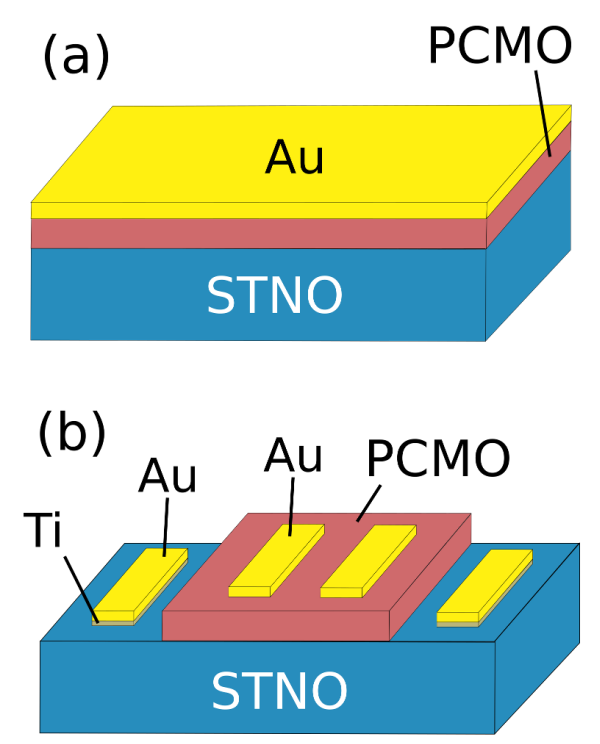

Figure 3.1: Sketch of the PCMO-STNO samples. (a) with unstructured PCMO layer and front side contact, (b) with structured PCMO layer, front and back side contacts

\subsubsection{Si sample}

The sample with the Si-based p-n junction was prepared by Hendrik Spende using thermal vapor deposition [114]. A multicrystalline n-doped $\left(N_{D}=10^{16} \mathrm{~cm}^{-3}\right)$ Si sample with thickness $150 \mu \mathrm{m}$ is used. First, the sample surface is cleaned by several organic solutions and a solution of $4 \% \mathrm{HF}$ in deionized water. Directly after the HF treatment, the sample is put into an evacuated evaporation chamber. Al wire positioned in a $\mathrm{W}$ coil is heated and thus evaporated onto the Si sample in an atmosphere with pressure $10^{-5}$ mbar. A total Al layer thickness of about $1 \mu \mathrm{m}$ is deposited. Afterwards, the sample is heated in $\mathrm{Ar}$ atmosphere, using a temperature curve with maximum temperature $592^{\circ} \mathrm{C}$. This is done in order to form a liquid layer between the Al layer and n-doped $\mathrm{Si}$ substrate. During cooling back to room temperature, the liquid layer recrystallizes and forms a $p^{+}$ Al-doped Si layer (doping $10^{18} \mathrm{~cm}^{-3}$ ) with a thickness of about $200 \mathrm{~nm}$ between the $\mathrm{Al}$ layer and the Si substrate. Additionally, the $\mathrm{Al}$ layer acts as the front side contact of the p-n junction. Finally, the back side contact is rubbed onto the back side of the $\mathrm{Si}$ substrate, using a $\mathrm{Al}$ bar coated by liquid $\mathrm{Ga}$, which forms a metallic GaAl layer at room 
temperature. Both contact layers act as Ohmic contacts on the respective layers.

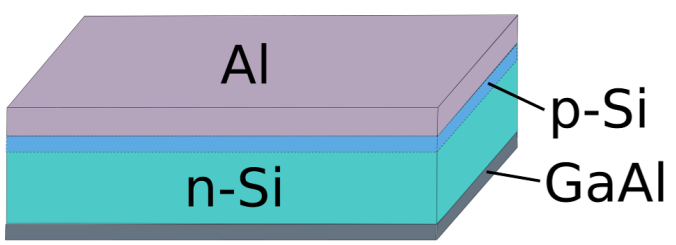

Figure 3.2: Si sample with deposited $\mathrm{Al}$ layer after heating. The p-Si layer is formed from $\mathrm{Au}$ diffusion into the Si during heating. Al forms the front side contact, while the rubbed GaAl layer is used as back side contact.

\subsection{Focused Ion Beam}

A Focused ion beam (FIB) device was used to prepare cross-section lamellas in the samples, with the option to move the lamella out of the original sample and further thin it afterwards for use on TEM sample holders. The used device in this work is a FEI Nova NanoLAB 600. It is a dual beam device, i.e. a combined scanning electron and scanning ion microscope. The electron and the ion beam source and lens systems are tilted by 52 degrees. The sample stage can be translated in three directions, rotated by 360 degrees and also tilted from -10 to +60 degrees. A field emission electron gun and a liquid gallium

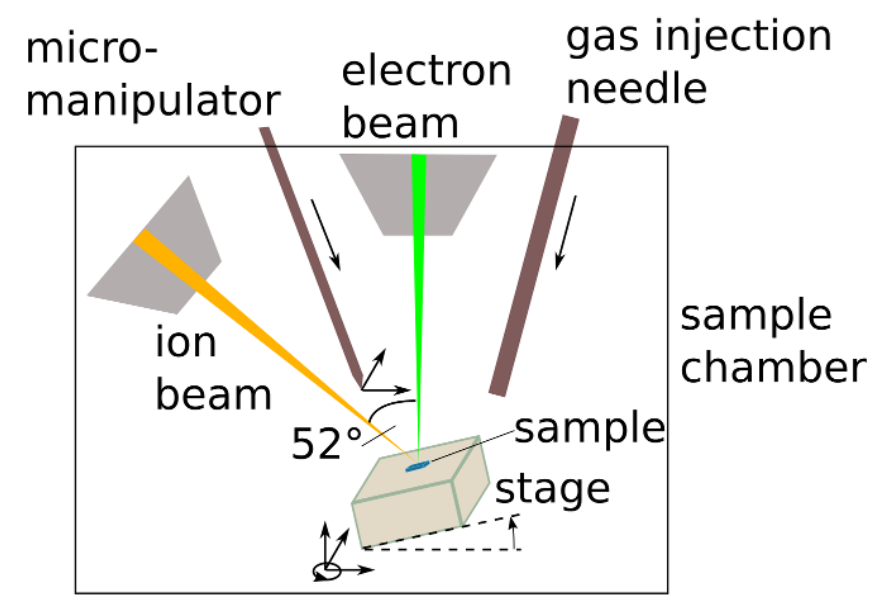

Figure 3.3: Sketch of the sample chamber in a Focused ion beam (FIB) device. The sample on the stage can be moved in all directions, rotated and tilted in a fixed direction. The electron and the ion beam have a fixed angle of 52 degrees. Micromanipulator and gas injection needle can be inserted as needed.

source are used as electron and ion sources, respectively. Like electrons, ionized Gallium atoms from the source are focused by electromagnetic lenses. While the electrons can be focused to obtain a spatial resolution of $1-2 \mathrm{~nm}$, depending on the used acceleration voltage and detector, ions can only achieve a resolution of $7 \mathrm{~nm}[115]$. On the other hand, the number of secondary electrons generated by a Ga ion is significantly larger than that of an electron, providing a better contrast. While the electrons are primarily meant for damage free sample investigation, the ions transfer a significantly higher energy to the 
sample, resulting in atom kick out. Continuous ion bombardment is usually used to dig into the sample, or clean surfaces with grazing ion beam incidence, with the focused and translatable beam providing the opportunity to dig trenches of arbitrary shape into the sample or even cut off thin portions. Electrons as well as ions can be accelerated with voltages up to $30 \mathrm{kV}$ in the device. The usable acceleration voltages range from 0.5 to $30 \mathrm{kV}$ and from 5 to $30 \mathrm{kV}$ for electrons and ions, respectively.

Furthermore, metallic and insulating layers can be deposited in-situ in the device. The basis for this is the insertable and translatable injection tube, called needle, of a gas injection system, from which a gas is flowing onto the sample surface. The electron or ion beam interacts with the gas, cracking gas molecules. The gas is designed in a way that cracked gas molecules will deposit onto the sample areas which are scanned by the electron beam during the cracking process. In that way, layers can be deposited on the sample, for example to protect sensible areas from ion bombardement. In this work, a Pt-C gas mixture was used to deposite layers containing mainly carbon with about $10 \%$ of platinum, making them nicely conductive.

The set of tools is complemented by an insertable micromanipulator tip, called Omniprobe, which is about $1 \mu \mathrm{m}$ in diameter at the apex. Like the gas injection needle, it can be translated in order to reach the desired location on the sample. After carefully driving the Omniprobe into mechanical contact to the sample, a mechanical and electrical connection can be formed by depositing a small Pt-C patch. A common practice to transfer thin lamellas from the sample to a sample holder, for example for use in a TEM, is to first dig out the lamella with the ion beam, then attach the Omniprobe to the lamella, afterwards cut the connection of the lamella to the sample with the ion beam, then move the Omniprobe to move the lamella away from the sample, drive it to the sample holder and attach it there by Pt-C layer depostion, and finally cut the Omniprobe from the lamella by ion beam to release it.

Common issues during the lamella preparation are charging and ion implantation. Charging is directly visible in the secondary electron images by distortions, altering the image and therefore obscuring the image of reality for the user. Charging occurs on insulating samples, or sample surface layers, and is often enhanced by driving the Omniprobe or the gas injection needle close to the sample surface. It can be prevented by providing additional conducting channels to the sample ground, for example by silver paint treatment or copper stripes attached to the sample surface. Another method is to drive the Omniprobe in electrical contact to the sample, which usually leads to the charging distortions to disappear. An even more serious issue is ion implantation. Fast Ga ions are implanted into the sample during the ion beam cutting and digging process. This effect is strongly increasing with the Ga ion acceleration energy. Aside from changing the chemical composition, Ga ion implantation often leads highly resistive layers to decrease their resistance, even to the point that layers considered insulating become clearly conductive, which can lead to unwanted short circuits.

If lamella surfaces are treated with the ion beam, they might be considerably affected by ion implanation. An iterative surface cleaning procedure, with decreasing ion beam energy or current with every step, can largely remove the affected layers, however it is still advisable to use additional low energy ion beam mills after the FIB treatment in order to ensure optimally clean and short circuit free lamella surfaces.

A detailed description of the cross-section lamella preparation procedure is given in section 3.8 . 


\subsection{NanoSEM with cooling stage}

In addition to the NanoLab dual beam instrument, an environmental SEM, type FEI NanoSEM 650, was used for SEM-based EBIC measurements. It is comparable to the SEM part of the NanoLab, however it is slightly optimized for use in the low voltage, low current regime. Furthermore, it enables a more versatile use of microscope add-ons. In this work, a Gatan model C1002 cooling stage is used for EBIC contacting and insitu cooling, which can be mounted to the SEM vacuum chamber wall. It is composed of a brass connector stage for installation between the sample holder and the stage, holding two heating contacts, a temperature sensor as well as vacuum-stable plastic tubes connected to a cooling circuit as described in Fig. 3.4. Liquid nitrogen is used in the cooling circuit to cool the sample, while an electric current is used to heat up the sample against the nitrogen cooling in order to establish an arbitrary stable temperature between 90 and $470 \mathrm{~K}$ [116], which is regulated by a Gatan SmartSet Model 904 Cold Stage Controller. After the set temperature is reached, which can take about 20-30 minutes, it stays stable within a range of about $1 \mathrm{~K}$ as long as the nitrogen flow stays constant. The measurement error at the temperature sensor is about $0.5 \mathrm{~K}$ [116]. The cooling circuit is essentially composed of a liquid nitrogen tank and a dewar vessel. At the exit of the tank, the outflow rate can be controlled by a rough and a fine valve. The dewar vessel contains a cryogenic spiral tube connected to the tank and to the tubes in the sample holder. The spiral is housed in a liquid nitrogen bath, enabling the rapid cooling process of the sample holder. After passing through the sample holder, the nitrogen exits the cooling circuit through an exit hole in the tube outside the microscope. The flow rate can conveniently adjusted small enough that about $1 \mathrm{~kg}$ of liquid nitrogen per hour is used while running a cooling experiment.

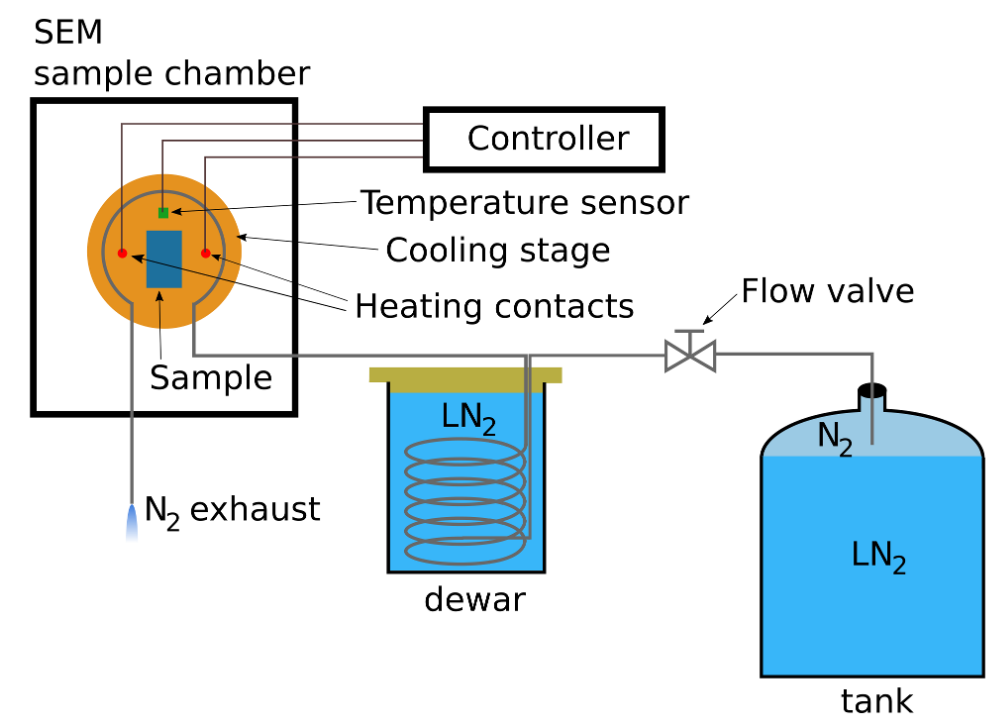

Figure 3.4: Sketch of the cooling setup for the NanoSEM microscope, modeled after 117. Gaseous nitrogen, which is cooled in the dewar, flows through the cooling stage, cooling the sample to about $90 \mathrm{~K}$. The temperature is regulated via two heating contacts in the stage, which are controlled automatically. The nitrogen flow rate can be adjusted by a valve. 


\subsection{SEM EBIC setup}

To measure EBIC, standard EBIC equipment provided by Gatan is used, with an additional measurement board to enable quantitative current measurement. It is compatible to the NanoLab as well as the NanoSEM microscope.

The standard sample holder is sketched in Fig. 3.5. The sample is fixed with silver paint on its backside to the sample holder. If no insulating layer is present between front and back contacts, the backside connection of the sample is provided by this silver paint contact. Otherwise, the backside contact is established on the sample top from an area separate to the front side contact. This is the case for the PCMO-STNO EBIC samples for SEM used in this work. Sample top contacts are established by $40 \mu \mathrm{m}$ thick gold wires, also using small silver paint contacts which are painted by a fine paintbrush with two bristles. The wires are connected with silver paint to larger isolated copper wires. These copper wires are mounted into gold tube plugs in an insulating ceramics piece at the edge of the sample holder, which are separated from each other. Up to four plugs can be used for this holder design, however only two are used in this work, as two-probe current measurements are performed. A rod can be screwed into the side of the sample holder in order to transfer it to the microscope with the sample already attached easily.

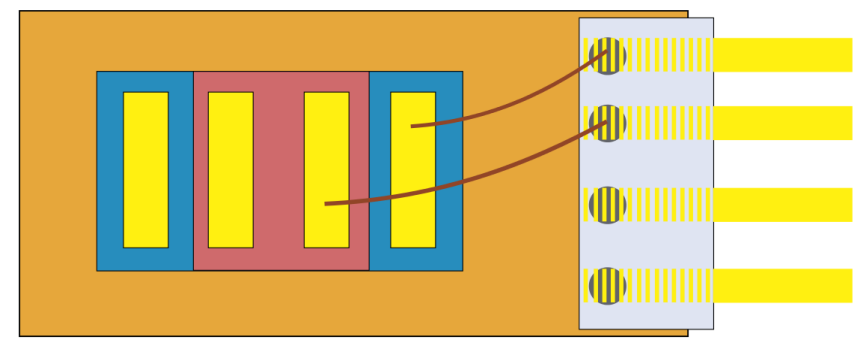

Figure 3.5: Sketch of the sample holder for EBIC in SEM. The sample, illustrated with the four contacts like in the structured PCMO-STNO sample, is fixed on the conductive holder, while the front side and back side contacts on the sample surface are connected to individual Au rods used for signal collection. The rods are isolated from each other and the main holder.

As an optional part of a sample holder, a 45 degree copper wedge can be screwed into the holder, where the sample can be mounted instead of directly on the holder, which leads to the sample being 45 degrees tilted from the holder axis (see Fig. 3.6). This wedge provides the advantage that the restricted tilt of the microscope sample stage (up to 60 degrees) can be compensated, so that the p-n junction in the sample can be positioned almost 90 degrees tilted to the electron beam axis. If a cross section lamella is prepared inside the sample, the full 90 degrees rotation cannot be used, as the lamella would be shadowed by the sample bulk. As a compromise, a tilt of 85 degrees is used in this case. Another EBIC sample holder was used for the Si sample investigations. The Si sample was fixed with silver paint onto a standard Al sample holder for SEM investigations, which was then screwed into the EBIC holder. This custom EBIC holder uses a metal spring to contact the front side, while the back side contact is ensured by the silver paint connection to the conductive $\mathrm{Al}$ sample holder. The disadvantage of this holder is that it does not allow to use a wedge to pre-tilt the sample, which is why the Si EBIC exper- 
iments were conducted under a tilt angle of only 52 degrees.

The sample is connected to the microscope stage via an insulated connector stage, which varies slightly for both microscope types: While in the NanoLAB the connector stage is fixed to the microscope and can be screwed into the stage for EBIC experiments as needed, in the NanoSEM the connector stage is part of the cooling setup and has to be mounted into the side vacuum chamber entry for each EBIC measurement. The plugs of the sample holder are connected to BNC connectors at the microscope vacuum chamber wall. The two point EBIC geometry is configured in a way that the two wires are connected to the core and shield of a single BNC connector, respectively.

(a)

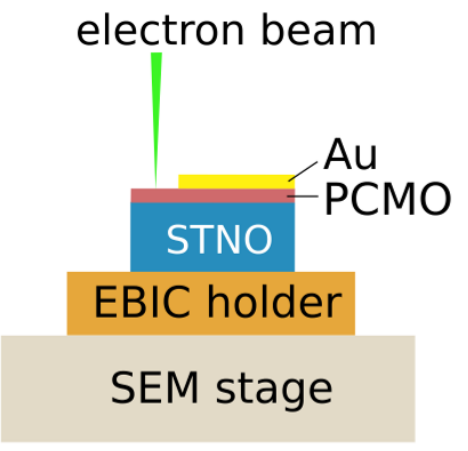

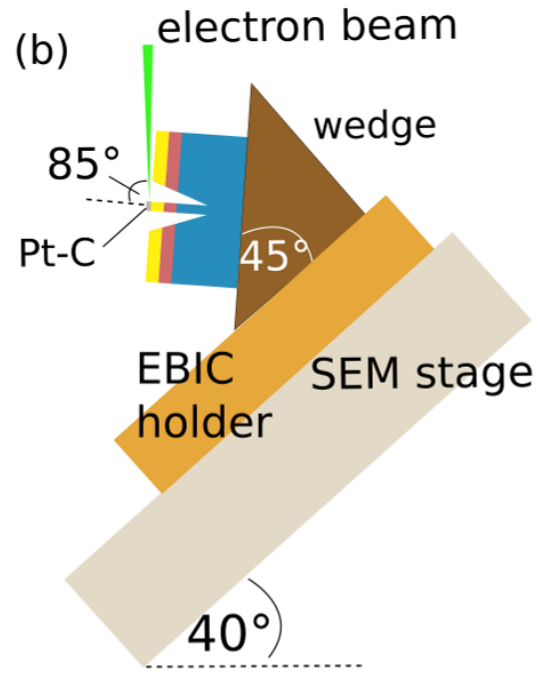

Figure 3.6: Sketch of the sample geometry in EBIC measurements of PCMO-STNO samples. (a) top view measurement, (b) cross section transmission (STEBIC) measurement, where the sample includes a processed lamella, protected by a FIB-deposited Pt-C layer, and is tilted by 85 degrees to the top view position. The copper wedge is necessary for a near cross section measurement as the SEM stage can only be tilted by 52 degrees.

Single-shielded BNC cables are used to connect the devices of the EBIC setup, which are identical for both microscopes. The setup of the devices is shown in Fig. 3.8. The devices contained in the setup, aside from the microscope itself, are the current preamplifier, the buffer voltage amplifier and the measurement board. The primary device is the preamplifier, which is connected to the specimen current output. As preamplifier, a Stanford Research SR570 model is used, which is powered by an internal rechargeable battery during measurements. It amplifies the specimen current by a given factor, and provides a voltage output, therefore also acting as a current-voltage converter. The amplification factor can be chosen in a range from $1 \mathrm{~mA} / \mathrm{V}$ to $1 \mathrm{pA} / \mathrm{V}$. The amplifier output is fed through the buffer amplifier and in the end connected to the microscope at its external detector port. This allows direct synchronization of all detector signals during recording, for example a secondary electron image can be synchronized with an EBIC image. Using the buffer amplifier, brightness and contrast of the EBIC image can be adjusted in order to fill the image contrast limits as needed.

An alternative signal path from the amplifier output is realized by a T-piece BNC connector in order to allow quantitative EBIC measurement beside the qualitative EBIC image recording. From the amplifier output BNC, the core and the shield signal are fed 

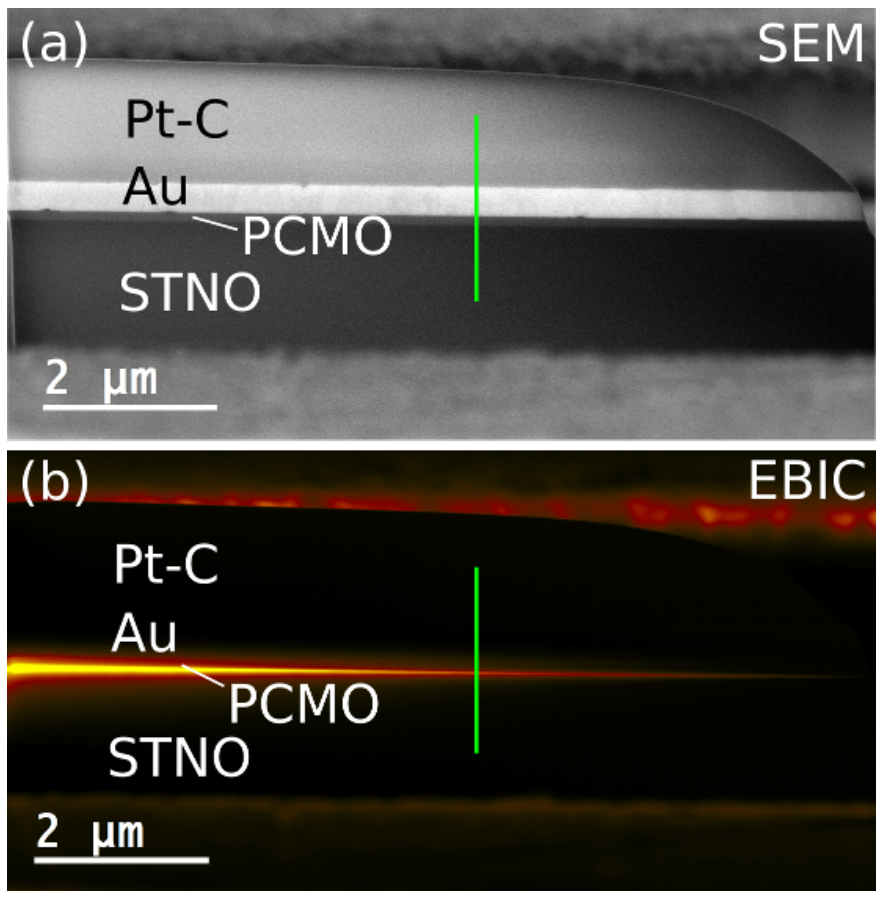

Figure 3.7: Example STEBIC measurement on a cross section PCMO-STNO lamella with a wedge shape, i.e. decreasing thickness from left to right. (a) SEM image. The grey unfocussed region is from the sample region at the edge of the trenches where the lamella was dug. (b) Corresponding EBIC image. The green line indicates the direction of STEBIC profiles which are discussed extensively in this work.

directly into a Meilhaus RedLab PMD-1008 measurement board, where they are digitalized with 8 bit and measured differentially. Furthermore, the output of the electron beam scanning coils are split off and also fed into the measurement board differentially. EBIC and beam position signal are continuously recorded by the measurement board during the image recording. With the three used measurement channels, the RedLab board is able to measure each signal with a frequency up to $300 \mathrm{~Hz}$. The signals can used to calibrate the EBIC image after the measurement, see section 3.9.1.

\subsubsection{EBIC preamplifier limitations}

In the EBIC setup used for SEM-based measurements, the Stanford Research System SR570 low noise current preamplifier is the most important component, as it makes the small currents in the $\mathrm{nA}$ or even $\mathrm{pA}$ range collected from the sample measureable by converting them to voltages with a considerable gain, so that they can be collected by common multimeters and the external detector ports of the microscopes. The amplifier is especially designed to collect these signals with the lowest noise possible, and provides a suitable frequency response for relatively slow SEM measurements. However, for quantitative EBIC measurements, in the sense that the absolute size of the collected current becomes important, some limitations of the amplifier have to be taken into account.

The amplifier provides a larger number of calibrated gain settings, called sensitivity. Each one provides a given gain, given in $\mathrm{nA} / \mathrm{V}$, with an error of $0.5 \%$ of the output signal [118]. This is necessary for flexible use, as the amplifier may only output a voltage within in the range from -5 to $+5 \mathrm{~V}$. Additionally, the gain may be changed to an uncalibrated 


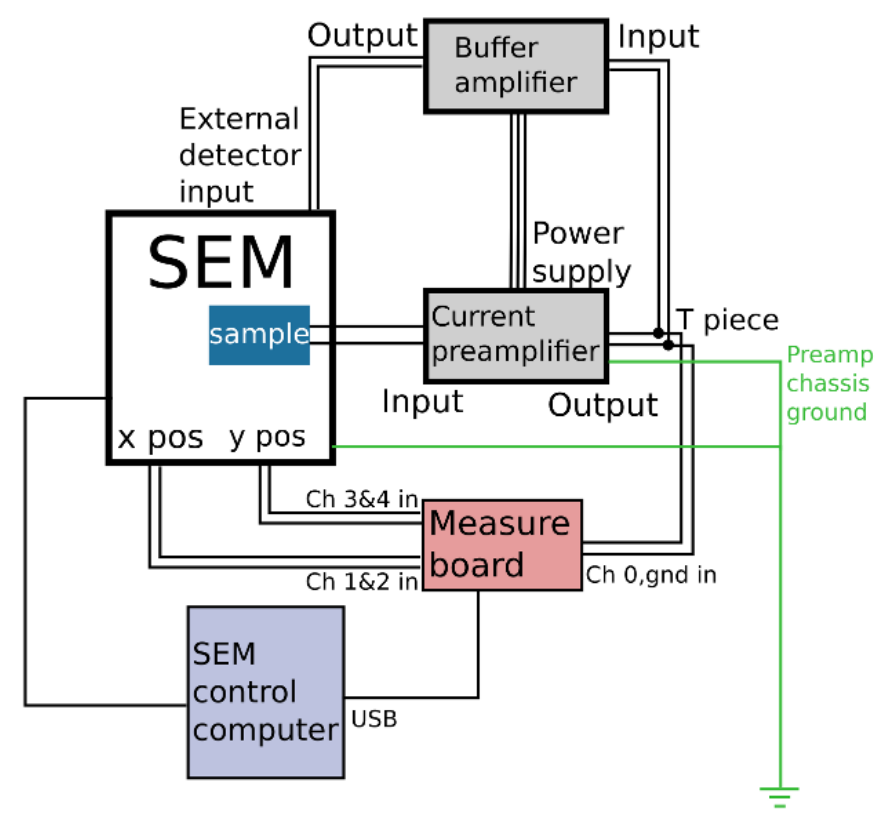

Figure 3.8: Sketch of the setup used for EBIC measurements in the SEM NanoLab 600 and NanoSEM 650. All double connections are provided by single-shielded BNC cables. The sample current is converted to a voltage by the preamplifier. The output of the current preamplifier is split at the $\mathrm{T}$ piece in order to simultaneously record an EBIC image via the SEM external detector input and measure the signal quantitatively via the measurement board. Signal indicating the $x$ and $y$ beam position is also fed to the measurement board. While the microscope and the preamplifier chassis are grounded, the sample and the preamplifier circuit are kept at floating potential.

setting providing the user with the opportunity to choose an arbitrary gain, which has to be calibrated by some external means. However, in general each calibrated sensitivity setting uses resistors with a different DC input impedance (which will be called input resistance $R_{i}$ in the following) and is only able to clearly process input signal with a given $3 \mathrm{~dB}$ bandwidth. The manual of the amplifier specifies these two properties for each sensitivity order of magnitude, i.e. from $10^{-3}$ to $10^{-12} \mathrm{~A} / \mathrm{V}$. Unfortunately, it is not clearly described in the manual which settings the in-between sensitivity use. For each order of magnitude, three sensitivity settings are available. For example in the order of magnitude $10^{-7} \mathrm{~A} / \mathrm{V}$, the sensitivities 100,200 and $500 \mathrm{nA} / \mathrm{V}$ are available. Still, when switching through the sensitivity settings, a distinct click sound may be heard between the $1 \cdot 10^{-n}$ and the $2 \cdot 10^{-n} \mathrm{nA} / \mathrm{V}$, for most $n$. This is interpreted is a switching in relays within the amplifier, which might for example change the used input resistor. Consequently, it is proposed that the sensitivity settings can be assigned to the sensitivity orders of magnitude as done in Table 3.1. This assignment is further supported by experimental results, as discussed in the following.

Fig. 3.9 shows results from EBIC measurements in the cross section as well as the top view geometry. Both measurement sets were carried out in the FIB using a $5 \mathrm{kV}$ electron beam. In the cross section measurement set (Fig. 3.9(a)), different electron beam dwell times were used, with a constant amplifier sensitivity setting. It can be concluded that for increasing dwell time, the EBIC profile gets more narrow, the maximum rises and the left and right end of the lines are more clearly approaching the same signal level, reduc- 


\begin{tabular}{r||l|r|r} 
Sens order & Sens settings & Bandwidth & $R_{i, a m p}$ \\
\hline $10^{-3} \mathrm{~A} / \mathrm{V}$ & $1 \mathrm{~mA} / \mathrm{V}, 500 \mu \mathrm{A} / \mathrm{V}, 200 \mu \mathrm{A} / \mathrm{V}$ & $1.0 \mathrm{MHz}$ & $1 \Omega$ \\
$10^{-4} \mathrm{~A} / \mathrm{V}$ & $100 \mu \mathrm{A} / \mathrm{V}, 50 \mu \mathrm{A} / \mathrm{V}, 20 \mu \mathrm{A} / \mathrm{V}$ & $500 \mathrm{kHz}$ & $1 \Omega$ \\
$10^{-5} \mathrm{~A} / \mathrm{V}$ & $10 \mu \mathrm{A} / \mathrm{V}, 5 \mu \mathrm{A} / \mathrm{V}, 2 \mu \mathrm{A} / \mathrm{V}$ & $200 \mathrm{kHz}$ & $100 \Omega$ \\
$10^{-6} \mathrm{~A} / \mathrm{V}$ & $1 \mu \mathrm{A} / \mathrm{V}, 500 \mathrm{nA} / \mathrm{V}, 200 \mathrm{nA} / \mathrm{V}$ & $20 \mathrm{kHz}$ & $100 \Omega$ \\
$10^{-7} \mathrm{~A} / \mathrm{V}$ & $100 \mathrm{nA} / \mathrm{V}, 50 \mathrm{nA} / \mathrm{V}, 20 \mathrm{nA} / \mathrm{V}$ & $2 \mathrm{kHz}$ & $10 \mathrm{k} \Omega$ \\
$10^{-8} \mathrm{~A} / \mathrm{V}$ & $10 \mathrm{nA} / \mathrm{V}, 5 \mathrm{nA} / \mathrm{V}, 2 \mathrm{nA} / \mathrm{V}$ & $200 \mathrm{~Hz}$ & $10 \mathrm{k} \Omega$ \\
$10^{-9} \mathrm{~A} / \mathrm{V}$ & $1 \mathrm{nA} / \mathrm{V}, 500 \mathrm{pA} / \mathrm{V}, 200 \mathrm{pA} / \mathrm{V}$ & $15 \mathrm{~Hz}$ & $1 \mathrm{M} \Omega$ \\
$10^{-10} \mathrm{~A} / \mathrm{V}$ & $100 \mathrm{pA} / \mathrm{V}, 50 \mathrm{pA} / \mathrm{V}, 20 \mathrm{pA} / \mathrm{V}$ & $10 \mathrm{~Hz}$ & $1 \mathrm{M} \Omega$ \\
$10^{-11} \mathrm{~A} / \mathrm{V}$ & $10 \mathrm{pA} / \mathrm{V}, 5 \mathrm{pA} / \mathrm{V}, 2 \mathrm{pA} / \mathrm{V}$ & $10 \mathrm{~Hz}$ & $1 \mathrm{M} \Omega$ \\
$10^{-12} \mathrm{~A} / \mathrm{V}$ & $1 \mathrm{pA} / \mathrm{V}$ & $10 \mathrm{~Hz}$ & $1 \mathrm{M} \Omega$
\end{tabular}

Table 3.1: Sensitivity orders of magnitude of the SR570 preamplifier, with the proposed assignment of individual sensitivity settings and the properties described in the manual. The bandwidth, defined for $3 \mathrm{~dB}$, is generally broader when using "High Bandwidth" instead of "Low Noise" mode, at the cost of higher noise. $R_{i, a m p}$ is the amplifier DC input impedance.

ing leakage effects. For the dwell times 300 and $500 \mu \mathrm{s}$, there is no considerable change, hinting at a saturation starting at $300 \mu \mathrm{s}$. The same behaviour has been found for many different measurements, acceleration voltages and beam currents, within this sensitivity order of magnitude (settings 100, 50 and $20 \mathrm{nA} / \mathrm{V}$ ) and sensitivity setting (Low Noise). Extending the measurements to whole images instead of profiles, a blur in the scanning direction determined by the dwell time is found. The probable reason for this behaviour is the limited bandwidth of the amplifier at these settings. According to Table 3.1, the bandwidth at these settings is $2 \mathrm{kHz}$, corresponding to a minimum sampling time of the processed signal of $\frac{1}{2 \mathrm{kHz}}=500 \mu \mathrm{s}$. As the $3 \mathrm{~dB}$ bandwidth is relatively broad, i.e. the actual minimum sampling time below which considerable signal changes become visible might be even slightly smaller, it is reasonable to conclude that the dwell time determines the signal sampling time and thus must be chosen to be larger or equal to the minimum sampling time allowed by the amplifier settings. It seems that a dwell time about a factor of 2 smaller than the indicated minimum sampling time is still reasonable for this kind of measurement, however it might not be if a higher precision is required, such as for smaller signal dynamics.

This conclusion becomes even more evident when choosing a higher sensitivity setting, as was done for the top view EBIC measurements in Fig. 3.9(b). Both shown images use the same high dwell time of $500 \mu \mathrm{s}$. They were performed on the same $\mathrm{MnO}_{z}$ precipitate on top of a PCMO layer. It is evident that at the higher sensitivity of $100 \mathrm{pA} / \mathrm{V}$, the image considerably blurs in the scanning direction determined by the dwell time (horizontal in the shown images). This can be explained by the strongly reduced bandwidth of this sensitivity setting, which is just $10 \mathrm{~Hz}$, corresponding to a minimum sampling time of $100 \mathrm{~ms}$. As the dwell time is much shorter than this time, the input signal of the amplifier cannot be processed properly.

Fig. 3.10 further elaborates on the influence of different sensitivity settings. Fig. 3.10(a) shows cross section and Fig. 3.10(b) shows top view EBIC measurements on PCMOSTNO p-n junctions. In the shown measurements, suitably high dwell times (500 $\mu$ s for the cross section and $300 \mu$ s for the top view measurements) were chosen in order to 

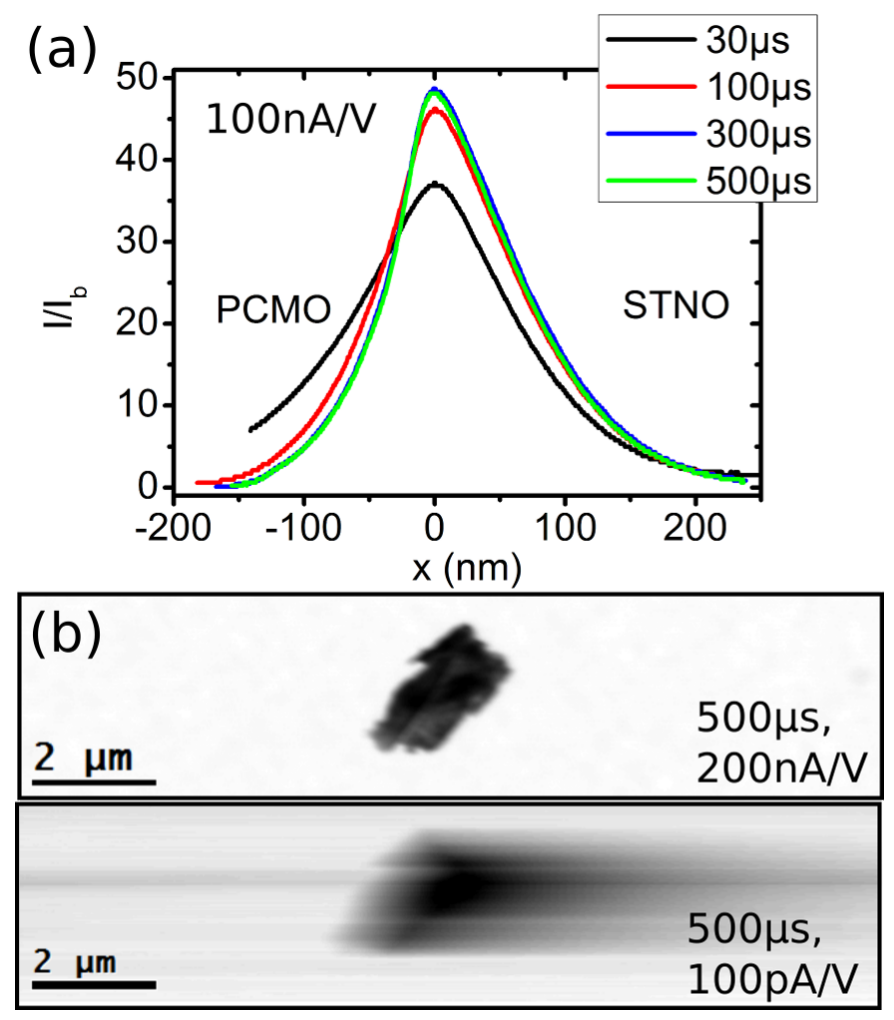

Figure 3.9: EBIC measurements, illustrating the effect of limited current amplifier bandwidth, or minimum sampling time. (a) $5 \mathrm{kV}$ cross section EBIC profiles for different electron beam dwell times, measured in cross section to the p-n junction. The used amplifier sensitivity $100 \mathrm{nA} / \mathrm{V}$ has minimum sampling time of $500 \mu \mathrm{s}$. If the dwell time is considerably lower than the minimum sampling time, the EBIC signal drops and broadens, i.e. EBIC images blur. (b) EBIC images of a $\mathrm{MnO}_{z}$ precipitate on top of a PCMO layer. The precipitate appears dark as it is not electrically active. Two different amplifier sensitivities have been used. While $200 \mathrm{nA} / \mathrm{V}$ has a minimum sampling time $50 \mu \mathrm{s}, 100 \mathrm{pA} / \mathrm{V}$ has $100 \mathrm{~ms}$. Thus the used dwell time of $500 \mu \mathrm{s}$ is much too low for the latter setting, leading to pronounced blur of the EBIC contrast.

exclude effects of the limited amplifier bandwidth. For both measurement sets, the sensitivity was varied between three sensitivity orders of magnitude $(2000 \mathrm{nA} / \mathrm{V}$ vs 1000 and $500 \mathrm{nA} / \mathrm{V}$ vs 100,50 and $20 \mathrm{nA} / \mathrm{V})$. Note that the measurements in the $\mu \mathrm{A} / \mathrm{V}$ range exhibit strong steps. These can be attributed to the low output signal (about 0.02-0.05 V), which approach the limit of the 8 bit analog-to-digital converter of the external detector input of the microscopes, resulting in clipping errors. The shape of each profile in a measurement set is very similar, however the height of the signal maximum is considerably reduced when changing from the low sensitivity settings of 2000 to $500 \mathrm{nA} / \mathrm{V}$ to the high settings of 100 to $20 \mathrm{nA} / \mathrm{V}$. The measurements done for high sensitivity may be aligned to the measurements for low sensitivity (dashed lines) by multiplying them by a correction factor, which is approximately 4 (4.1 for $5 \mathrm{kV}$ and 3.9 for $15 \mathrm{kV}$ measurements) for the cross section and 7.9 for the $5 \mathrm{kV}$ top view measurements. As can be extracted from Table 3.1, the amplifier input resistance changes from $100 \Omega$ for the low to $10 \mathrm{k} \Omega$ for the high sensitivity settings, and is constant within the low and high setting ranges. In conclusion, the limits of the preamplifier have to be taken into account in EBIC ex- 

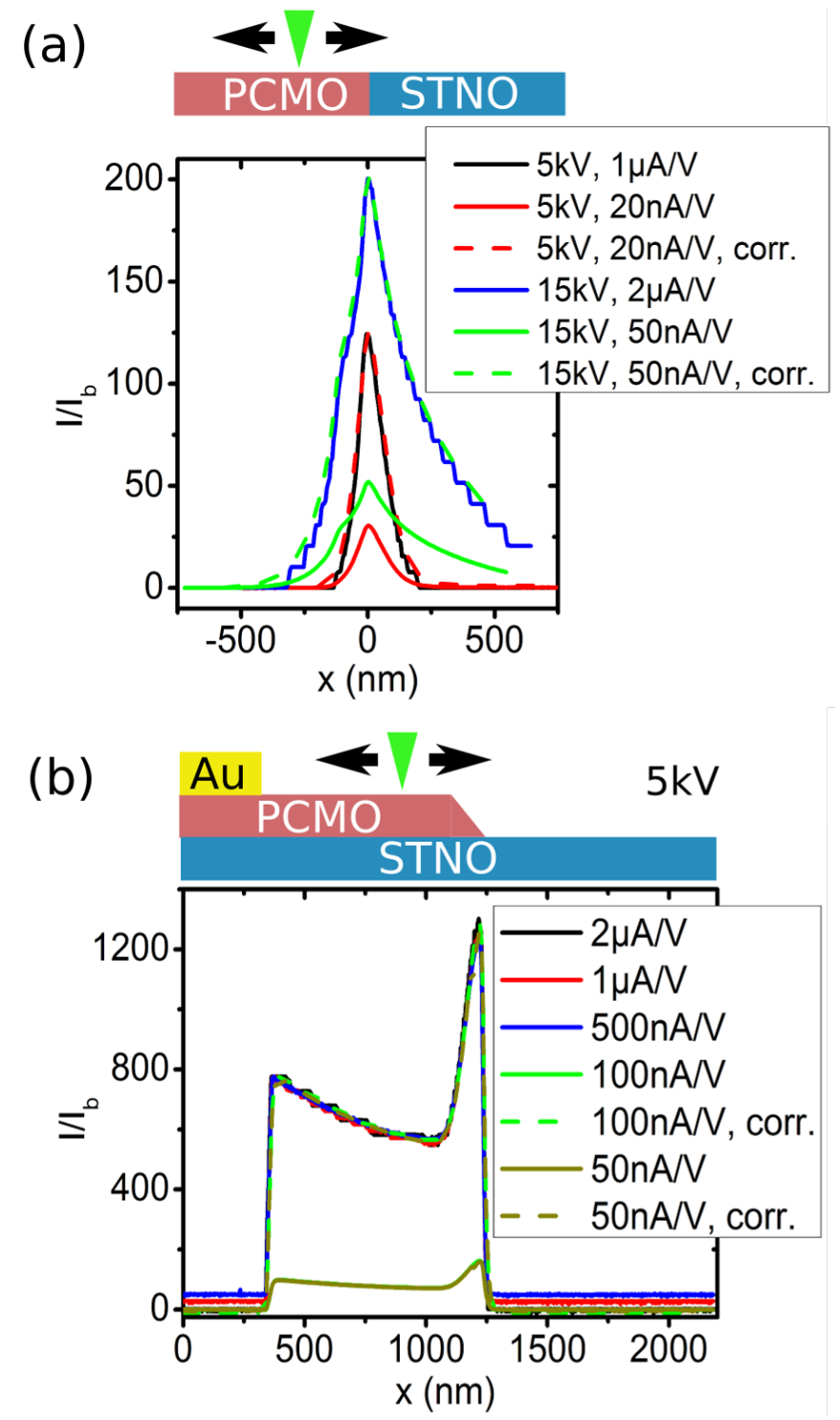

Figure 3.10: Variation of the current amplifier sensitivity for (a) cross-section $5 \mathrm{kV}$ and $15 \mathrm{kV}$ and (b) top view $5 \mathrm{kV}$ EBIC profiles. The used electron beam dwell time was high enough to exclude effects of limited bandwidth. For the high sensitivities 100-20 nA/V, the signal is much lower than for the low sensitivities 2000-500 nA/V. Still, the high sensitivity profiles can be aligned to the low sensitivity profiles by multiplying them with a correction factor of approximately 4 in (a) and 8 in (b). The disadvantage of low sensitivity shows up in the pronounced artificial steps in the profiles for $2 \mu \mathrm{A} / \mathrm{V}$, which are assumed to arise due to clipping effects from the microscope detector analog-to-digital converter. The notable signal peak in (b) at the edge of the PCMO layer is due to gradually reducing PCMO layer thickness, allowing a larger fraction of electron-hole pairs to be excited directly at the p-n interface.

periments. For each used sensitivity setting, the dwell time has to be chosen according to this setting's bandwidth limits. To avoid this problem, it may be useful to switch the amplifier from "Low Noise" to "High Bandwidth" mode, thereby enhancing the bandwidth, but also the measurement noise. Additionally, for high sensitivity settings a correction factor may be applied to EBIC measurements in order to obtain the real quantitative 
currents. Modeling this correction factor is be discussed in Sec. 8.4 .

\subsection{Titan TEM}

An FEI Titan 80-300 ETEM was used for the TEM-based EBIC experiments. It uses an XFEG field emission gun with high brightness and a standard energy spread of about $1 \mathrm{eV}$. The energy spread may be even further reduced to about $0.2 \mathrm{eV}$ by using the built in source monochromator, which is especially important to record ELNES structure. The TEM also uses a three lens condensor system, enabling the formation of a particularly parallel beam even in STEM mode. Furthermore, the TEM is equipped with a CEOS image aberration correction system, which may provide a spatial resolution of $80 \mathrm{pm}$ in HRTEM mode. Even though not using a probe aberration corrector to directly enhance STEM spatial resolution, the achievable probe size in STEM mode is $0.14 \mathrm{~nm}$. The microscope uses a bottom-mounted Gatan Image Filter (GIF) ER/965P for post-specimen beam analysis. This enables the recording of EELS spectra which may be accessed by FEI as well as Gatan software. The microscope is also equipped with a Lorentz lens, enabling the possibility of field free low magnification imaging, and an Oxford Instruments EDX detector. As a pretty unique feature, it also contains a flexible gas supply which may be used to flood the sample area with a gas with pressure up to 20 mbar.

\subsection{TEM EBIC setup}

The principle component in the EBIC setup for TEM is the sample holder. In this work, a DENSsolutions D7+ Lightning system is used, which is designed for simultaneous Joule heating and biasing via 6 electrical contacts. The sample carrier is a MEMS chip equipped with 6 contacts strips where the sample is transferred during FIB preparation. The MEMS chip is a Si piece with a silicon nitride layer, which is thinned to a membrane in the center, and to holes between individual contacts, so that the sample lamella can be mounted above a hole and acts as the connection between two or four contacts (two on each side). The chips are available in different geometries so that they can support 2-point sample resistance and 4-point temperature measurement, or vice versa, or just one of each.

Once inserted into the sample holder, the output from the chip contacts may be measured for each contact individually as the holder is connected to an interconnect box (IC box 1 in Fig. 3.12 supplied by the manufacturer. As the DENS setup is designed for biasing, applying currents for Joule heating and resistance measurement only, in this work a custom measurement setup was connected to the sample holder IC box. The setup includes a Keithley 6517A electrometer used for measuring the EBIC fed from two of the holder contacts to the electrometer triax input. Furthermore, the Keithley setup includes a 2430 sourcemeter, which is not used for the EBIC measurement. However, the connections may be changed to include the sourcemeter as a voltage source in a series connection with the sample holder and the electrometer. This enables the opportunity to measure current-voltage characteristics on a TEM sample. The electrometer and the sourcemeter are connected via a General Purpose Interface Bus (GPIB) plug, which is also connected to a measurement computer. All used devices are grounded using the common ground of the TEM. Furthermore, during the measurement the operator is also connected to the same ground using a leg connection. 


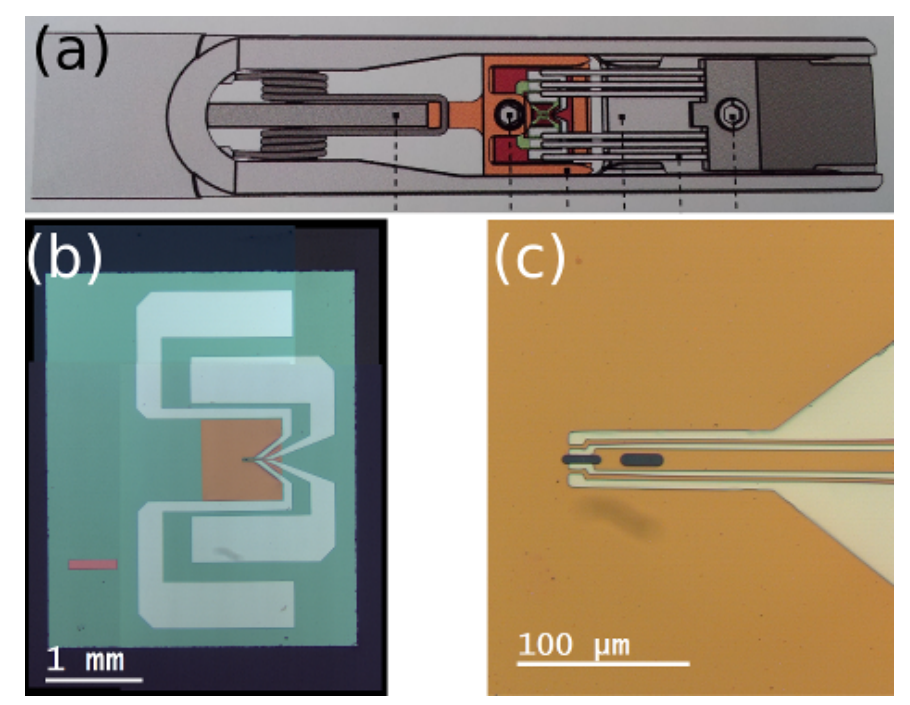

Figure 3.11: DENS D7+ Lightning system used for TEM-based EBIC. (a) sketch of the holder head containing a MEMS chip contacted via 6 metal rods, taken from [119]. (b) Light microscope image of a four contact MEMS chip used in this work, showing the contact strips (light blue) and the thinned membrane (orange). (c) Magnified chip part with the holes in the membrane where the sample lamella may be positioned.

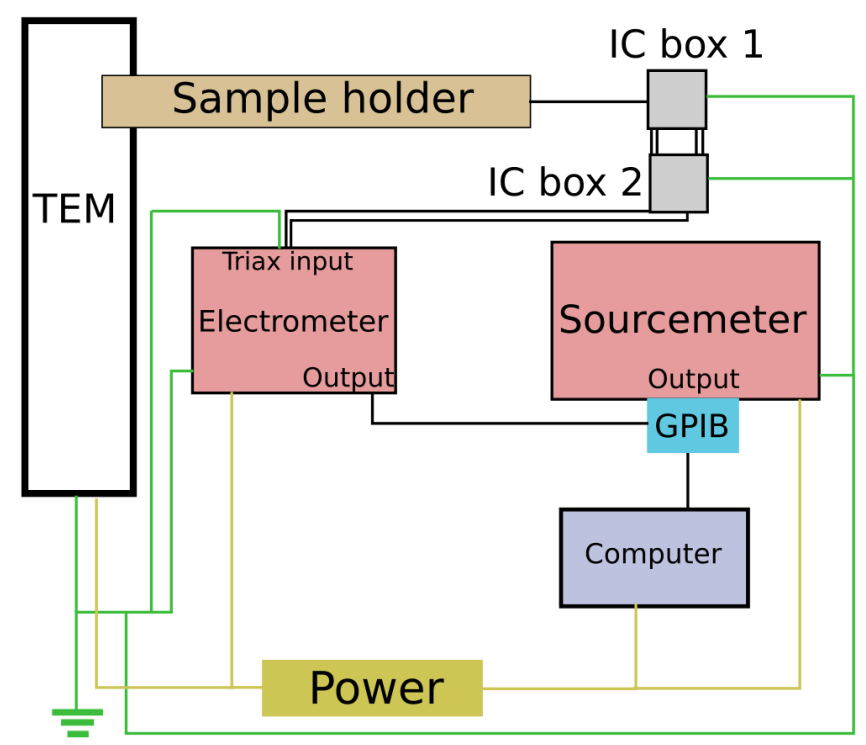

Figure 3.12: Sketch of the setup used for EBIC measurements in the TEM Titan 80-300. The sample current is fed through the sample holder. Using two interconnect (IC) boxes, the signal from the two used contacts is isolated and fed to the electrometer using single-shielded BNC cables. The electrometer signal is fed to the computer controlling the electrometer via a GPIB interface. The sourcemeter is just used to provide additional signal timestamps in this work, but may be connected in order to apply a bias voltage to the sample. All devices are connected to the same power source and ground.

The data from the electrometer and the sourcemeter are collected by a custom Labviewbased program, called VCC, which also supports applying automatic bias voltage control. VCC was written and maintained by Jonas Lindner [120]. The program controls 
the sourcemeter voltage source and provides output of measured voltage and current at the sourcemeter and the electrometer, respectively, as well as a time stamp signal from both devices for each measurement and a start time stamp. The time stamp was used to synchronize the EBIC measurement with the STEM image recorded by the microscope software, which provides a start and end timestamp of the image recording. This procedure is explained more detailed in Sec. 3.9 .2 .

\subsection{TEM holography setup}

The holography data was collected in the FEI Titan 80-300 Berlin Holography at the ZELMI lab at TU Berlin. The microscope was operated and the reconstruction of holograms performed by Dr. Tore Niermann. The experiment was supported by Prof. Dr. Michael Lehmann.

The used TEM is very similar to the Titan ETEM operated in Göttingen, for example it also contains an image aberration corrector, providing a spatial resolution of $75 \mathrm{pm}$ at $300 \mathrm{kV}$ acceleration voltage. However it lacks EDX and EELS systems for chemical analysis as well as the unique gas supply system. For conducting holography, it is equipped with 2 Möllenstedt biprisms and a holography transfer lens, as well as a Lorentz lens. The use of specialized holography analysis software developed by the ZELMI team enables the reconstruction of holograms with atomic resolution [104]. The conducted experiment provided the possibility to perform reconstructions with a spatial resolution of about $10 \mathrm{~nm}$. The sample used for holography was also slightly rethinned using a FEI Helios Nanolab 600 FIB from ZELMI lab.

\subsection{Focused ion beam preparation procedures}

The parameters used for focused ion beam (FIB) preparation are briefly compiled in the following.

\subsubsection{Cross section lamellas for SEM-based EBIC}

The sample was put on the 45 degree copper wedge which is screwed to the EBIC holder. A $500 \mathrm{~nm}$ thick Pt-C film was deposited on the sample area designated for the lamella, which was in the sample part covered by a Au contact. A $30 \mathrm{kV}$ ion beam acceleration voltage and $100 \mathrm{pA}$ beam current were used and the stage was tilted by 7 degrees in order for the ion beam to penetrate the sample perpendicular to its surface. The Pt-C film thickness was extended by $1.5 \mu \mathrm{m}$ using a larger beam current of $300 \mathrm{pA}$. The lamella was then dug out using a Regular Cross Section pattern on each lamella side, which uses a sequence of rectangular patterns. The front side pattern was rotated to dig on the back side (see Fig. 3.13(a)). Afterwards, the lamella was thinned with Cleaning Cross Section patterns, which feature a larger dwell time on each pattern line than the rectangular patterns, using a tilt offset from the perpendicular beam position for each side. The angle was increased $(+)$ for the front side and decreased $(-)$ for the back side. Using a $30 \mathrm{kV}$ ion beam, the beam current was successively decreased for each thinning step. Furthermore, the pattern was slightly reduced in the $x$ direction for each step in order to provide a more stable connection to the bulk sample. Finally, the lamella was cleaned with a $5 \mathrm{kV}$ ion beam using a beam current of $29 \mathrm{pA}$ and a large tilt offset of \pm 7 
degrees. A rectangular pattern was used in this step. The steps are detailed in Table 3.2. To produce a wedge shape lamella, the back side pattern during the thinning steps was rotated by 5 degrees, as shown in Fig. 3.13(b).

Similar preparation procedures were used to produce lamellas in PCMO-STNO as well as Si samples. For the Si sample, the standard automatic script for the digging and first thinning process, provided by FEI and modified by Volker Radisch at Uni Göttingen, was used with a target lamella thickness of $10 \mu \mathrm{m}$. The rest of the procedure with three additional thinning steps and a cleaning step was carried out similar to the procedure described in Table 3.2. Starting from the second thinning step, the wedge was prepared by tilting the back side thinning pattern.

\begin{tabular}{l||r|r|r|r|r|r} 
Step & $U_{\text {ion }}$ & $I_{\text {ion }}$ & Tilt offset & $x$ size & $y$ size & $z$ size \\
\hline Dig & $30 \mathrm{kV}$ & $7 \mathrm{nA}$ & $\pm 7^{\circ}$ & $17 \mu \mathrm{m}$ & $16 \mu \mathrm{m}$ & $8 \mu \mathrm{m}$ \\
Thin 1 & $30 \mathrm{kV}$ & $1 \mathrm{nA}$ & $\pm 1.5^{\circ}$ & $16 \mu \mathrm{m}$ & $2 \mu \mathrm{m}$ & $6 \mu \mathrm{m}$ \\
Thin 2 & $30 \mathrm{kV}$ & $300 \mathrm{pA}$ & $\pm 1.2^{\circ}$ & $14 \mu \mathrm{m}$ & $0.5 \mu \mathrm{m}$ & $0.8 \mu \mathrm{m}$ \\
Thin 3 & $30 \mathrm{kV}$ & $100 \mathrm{pA}$ & $\pm 1.2^{\circ}$ & $13 \mu \mathrm{m}$ & $1 \mu \mathrm{m}$ & $0.8 \mu \mathrm{m}$ \\
Thin 4 & $30 \mathrm{kV}$ & $30 \mathrm{pA}$ & $\pm 0.7^{\circ}$ & $13 \mu \mathrm{m}$ & $0.3 \mu \mathrm{m}$ & $0.8 \mu \mathrm{m}$ \\
Clean & $5 \mathrm{kV}$ & $29 \mathrm{pA}$ & $\pm 7^{\circ}$ & $13 \mu \mathrm{m}$ & $0.8 \mu \mathrm{m}$ & $0.01 \mu \mathrm{m}$
\end{tabular}

Table 3.2: Typical ion beam patterning steps in preparing the lamellas for SEM-based EBIC experiments, as shown in Fig. 3.13 The tilt offset on the lamella front $(+)$ and back side $(-)$ is from the ion beam penetration direction perpendicular to the sample surface. Regular Cross Section patterns are used for the digging, Cleaning Cross Section patterns for the thinning and Rectangular patterns for the cleaning steps.

\subsubsection{Cross section lamellas for electron holography}

For the cross section lamellas for TEM based off axis holography, a similar preparation method to that described in Table 3.2 was used. They were taken from the sample with an unstructured $\mathrm{Au}$ contact. After the digging and the first thinning step, the lamella was transferred to a standard $\mathrm{Cu}$ carrier grid. The other three thinning steps were performed on the grid. The thinned lamella, as shown in Fig. 3.14(a) was then additionally thinned in lamella top view, i.e. with ions penetrating the lamella in perpendicular direction to the layer interfaces. A beam current of $300 \mathrm{pA}$ was used, with a rectangle pattern of size $3 \times 0.8 \mu \mathrm{m}$. The reason for this was to produce a defined gap in the lamella where the unperturbed electron wave can be processed together with the object wave where electrons pass through the thinned lamella. Additionally, to ensure a defined short circuit across the p-n junction interface, two Pt-C layers were deposited with a pattern depth of $1.2 \mu \mathrm{m}$ at an ion beam current of $100 \mathrm{pA}$. The result is shown in Fig. 2.1(b). A final cleaning step was performed on-site with the Helios FIB before the holograms were recorded.

\subsubsection{Cross section lamellas for TEM-based EBIC}

Comparing to the previously described FIB preparation procedure, the one used for TEM-based EBIC lamellas is considerably more complex.

A large slice with a lateral size of about $100 \times 2 \mu \mathrm{m}$ was prepared from the sample with the unstructured Au contact. As the back side contact had to be prepared in an external 

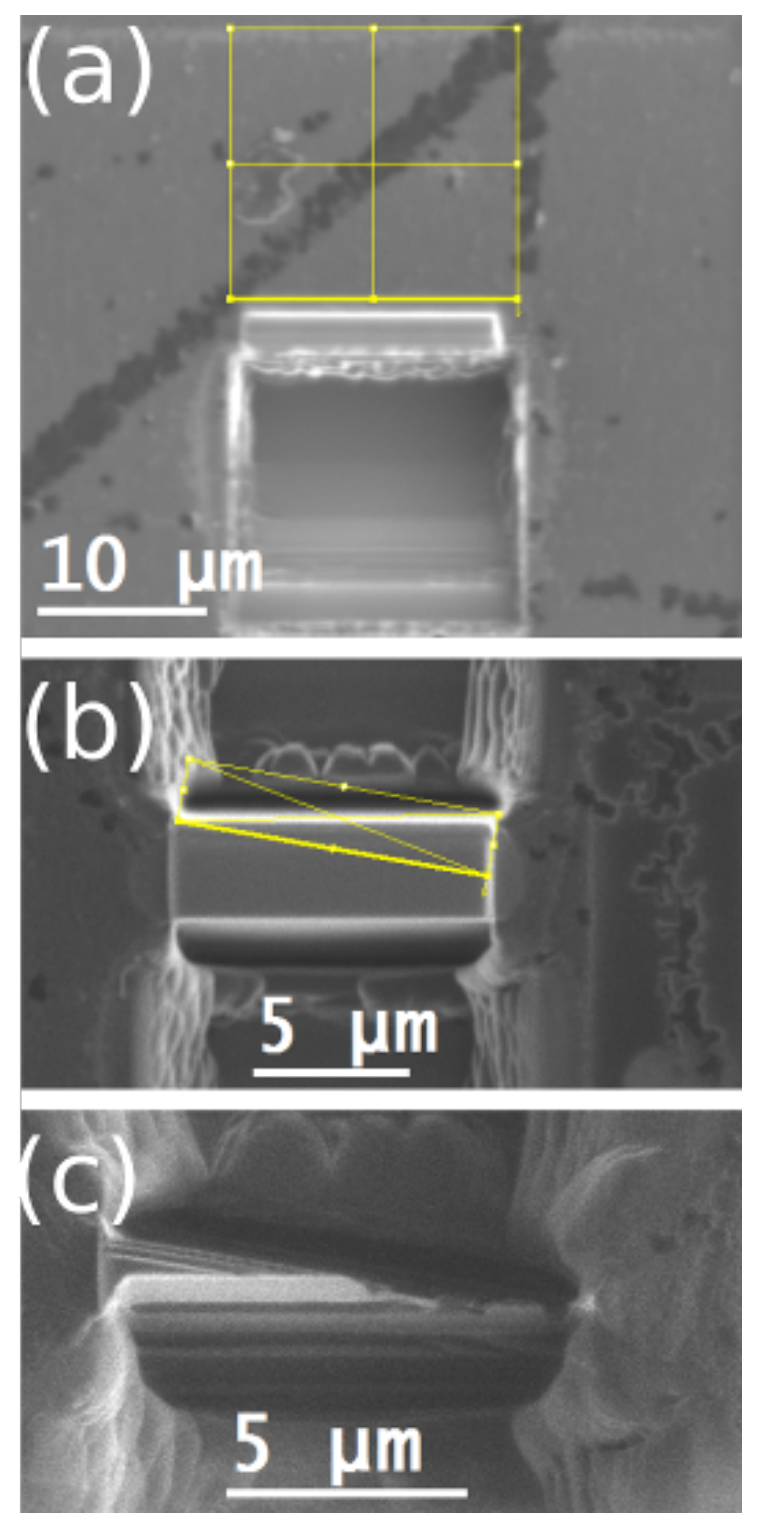

Figure 3.13: FIB preparation steps for wedge shaped cross section lamellas. Top view secondary electron images from ion beam scans are shown. (a) Trench digging already performed on the front side of the designated lamella, and pattern marked for digging on the back side. The lamella surface is protected by a deposited Pt-C film. (b) After the lamella is dug, a rotated pattern is used to thin the lamella back side in order to prepare the wedge. (c) Finished wedge shaped lamella.

sputtering setup directly onto the lamella, the aim of this strategy was to deposit a back side contact on the large slice so that several lamellas could be prepared out of the slice, with only one sputtering process. First, a protective $100 \mathrm{~nm} \mathrm{Pt-C} \mathrm{film} \mathrm{was}$ deposited with the electron beam under incidence perpendicular to the sample surface, at an acceleration voltage of $5 \mathrm{kV}$ and an electron beam current of $1.6 \mathrm{nA}$. The Pt-C film was extended to a thickness of $5 \mu \mathrm{m}$ by using the $30 \mathrm{kV}$ ion beam with a current of $300 \mathrm{pA}$, also with perpendicular incidence, i.e. stage tilted to 52 degrees. The digging was performed at the same stage angle with a Regular Cross Section pattern and ion beam current of $20 \mathrm{nA}$. The pattern size was $103 \times 20 \times 15 \mu \mathrm{m}$ on the front side and 

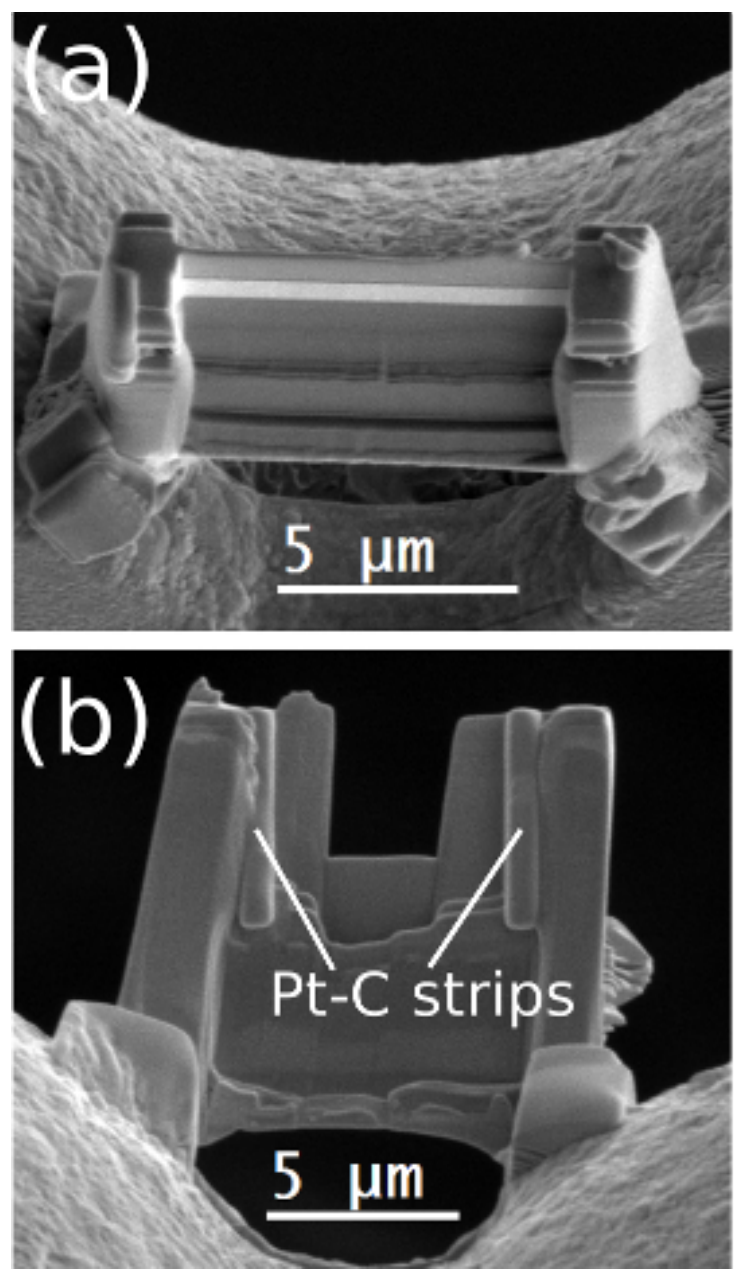

Figure 3.14: Altered FIB preparation for electron holography lamella. (a) Thinned lamella on $\mathrm{Cu}$ grid (inclined top view), (b) finished lamella with center gap and additional Pt-C strips, which ensure passage of an unperturbed electron wave next to the thinned lamella region and short circuit across the p-n junction, respectively (side view).

$103 \times 12 \times 7 \mu \mathrm{m}$. The asymmetric pattern sizes were necessary as the cut of the slice had to be performed at a non-perpendicular angle, thus needing more space on the front side. The first thinning step at a current of $1 \mathrm{nA}$ was performed with a Cleaning Cross Section pattern depth of $3 \mu \mathrm{m}$ at a tilt angle offset of \pm 1.5 degrees. The cut of the slice, as shown in Fig. 3.15(a), was performed from the back side with a current of $1 \mathrm{nA}$ under a tilt angle of -10 degrees, ensuring the cut to be as perpendicular to the slice surface as possible. After the cut, the slice was transferred to a standard $\mathrm{Cu}$ grid. The micromanipulator had to be fixed at the center of the slice due to its unusually long size.

On the grid, the part of the slice where the cut was performed was thinned away in order to obtain a cuboid slice, which is better suited for deposition on its surfaces. The thinning was performed from one the slice sides using a beam current of $1 \mathrm{nA}$ and a rectangle pattern. The produced back surface of the slice was then cleaned by a Cleaning Cross Section using a current of $300 \mathrm{pA}$. Turning the grid by 90 degrees using a custom horizontal grid holder, the lamella was cut from the grid with a current of $300 \mathrm{pA}$ and refixed on the other grid side, thereby turning the slice upside down. Returning the grid to its usual holder, the lamella was then cut again and transferred to a dummy sample. 
To ensure that no material of the latter would have to be included in lamellas, a "landing pad" for the slice was prepared by depositing a $5 \mu \mathrm{m}$ thick Pt-C film with an ion beam current of $300 \mathrm{pA}$. The upside down slice fixed on the dummy sample is shown in Fig. $3.15(b)$.

Afterwards, the slice was transferred to a sputtering chamber, where the back side contact was sputtered by Emanuel Franke. A Ti film, approximately $50 \mathrm{~nm}$ thick, was sputtered, followed by an approximately $300 \mathrm{~nm}$ thick protective $\mathrm{Au}$ film. The same layer materials were used for STNO ohmic contacts on the structured samples. Returning to the FIB, a Pt-C film with $8 \mu \mathrm{m}$ lateral size and $1.5 \mu \mathrm{m}$ thickness was deposited on a part of the slice with a $30 \mathrm{kV}$ ion beam under incidence perpendicular to the slice (backside) surface and a beam current of $100 \mathrm{pA}$. The protected part of the slice was cut under a tilt angle of 7 degrees, see Fig. 3.15(c) for an image of the lamella lift out process. The lamella was first transferred to a $\mathrm{Cu}$ grid, where a part of the Pt-C film on the front side originating from the "landing pad" was cut using a current of $100 \mathrm{pA}$, with the goal of obtaining a roughly cuboid lamella. The $\mathrm{Cu}$ grid was transferred to the horizontal grid holder, which was tilted to 13 degrees in order to transfer the lamella on the DENS chip under this angle. This is important in order to perform lamella thinning on the chip. The transfer to the chip was conducted while avoiding ion beam contact to the chip contacts as much as possible. Pt-C strips of $1 \mu \mathrm{m}$ thickness were deposited on both sides to fix the lamella on the chip contacts, using a small ion beam current of $10 \mathrm{pA}$ in order to avoid damage to the contacts.

The second thinning step (first on the chip) is then performed like described in Table 3.2 with an ion beam current of $300 \mathrm{pA}$. The back side thinning is performed twice, the second time with a Cleaning Cross Section rotated by 5 degrees to produce a wedge shaped lamella. After this cleaning step, gaps are thinned in the lamella sides in order to provide a clear current paths. The goal is to keep only the back side contact intact on one side and only the front side contact on the other side. The gaps are cut over the hole in the chip, at ion beam current $300 \mathrm{pA}$ and at 60 degrees tilt angle, which is as close to ion beam penetration from one of the lamella sides as possible. Afterwards, the third and fourth thinning step as well as the final cleaning step under a $5 \mathrm{kV}$ ion beam are performed as described in Table 3.2. The final result is shown in Fig. 3.15(d). 

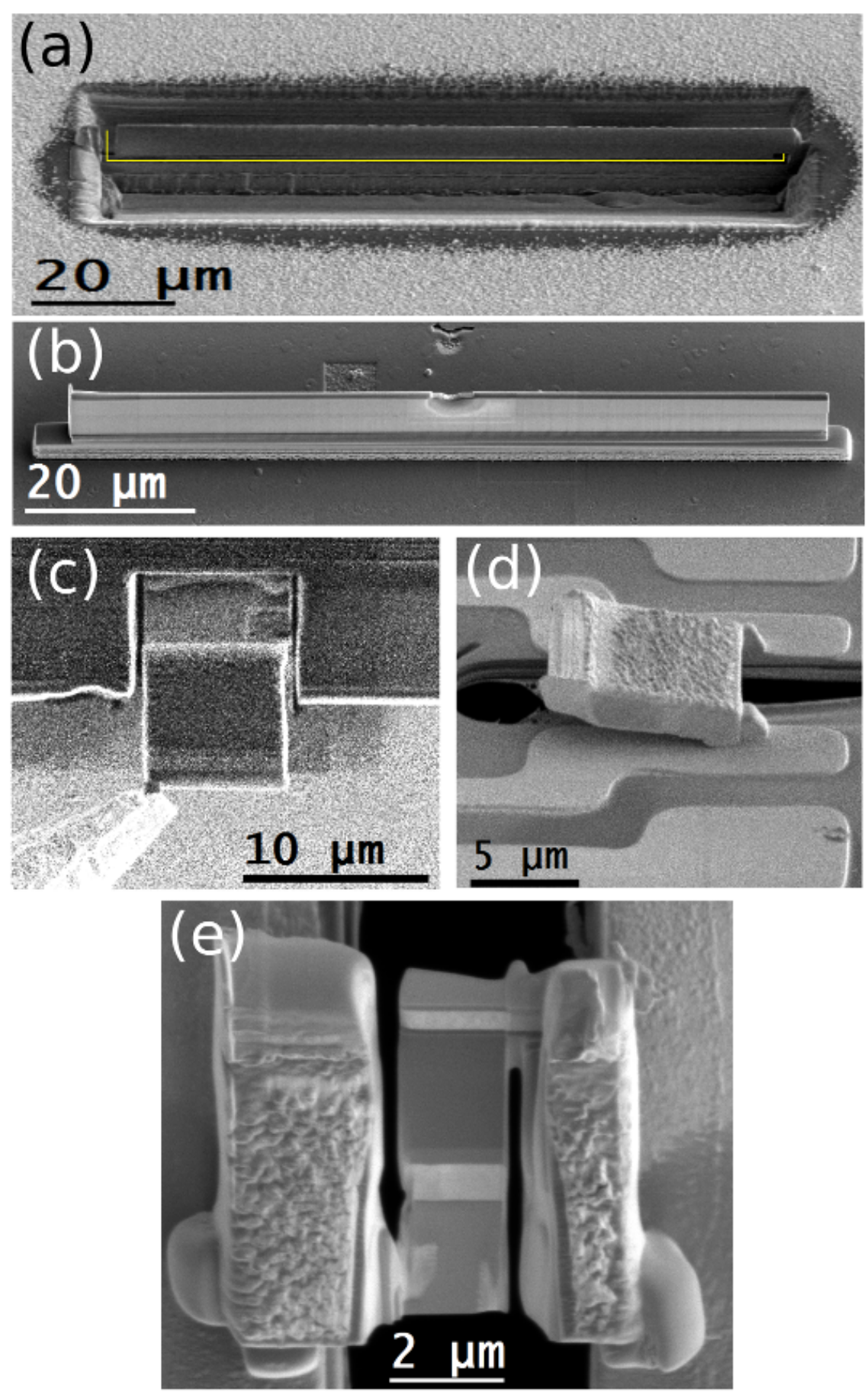

Figure 3.15: FIB preparation of lamellas for TEM-based EBIC experiments. (a) Cuts on the dug out large slice of the sample, (b) slice positioned upside down on a Pt-C "landing pad" on a dummy sample to enable back side contact sputtering, (c) lamella lift out from the slice using the Omniprobe micromanipulator, (d) lamella positioned on chip (side view) under tilt angle 13 degrees, (e) finished lamella with gaps to enable a clear current path across the p-n junction. 


\subsection{EBIC processing}

In order to obtain quantitative EBIC measurements, the raw EBIC data must be processed. The procedures are quite different for the SEM-based and the TEM-based data. The former requires quantification of the recorded qualitative EBIC images, while for the latter EBIC profiles have to be reconstructed from continuous quantitative measurements. The procedures for both are described in the following.

\subsubsection{Quantification of SEM-based EBIC images}

The recorded EBIC images are qualitative data, i.e. they are not calibrated to the actually measured current. As described in Section 3.4, quantitative data, i.e. the actual output data of the current preamplifier in Volts, are recorded along with EBIC images. As the maximum recording frequency of these data is $300 \mathrm{~Hz}$ and dwell times of less than $1 \mathrm{~ms}$ are often used in EBIC measurements, the quantitative data cannot directly be used to reconstruct an EBIC image. However, they may be used to calibrate the EBIC images in order to make them quantitative. The calibration procedure is performed by custom DigitalMicrograph scripts and is briefly explained in the following.

The quantitative data always include a section recorded before the actual image recording, where the electron beam is blanked. This is called the dark current. The scripts allow the selection of this section. The average of the selected data is subtracted from the data recorded during the beam scanning process. Afterwards, the amplifier sensitivity is multiplied to the data in order to transform the measured voltage to a current signal. The actual calibration of the qualitative (image) data is done by comparing the cumulative histograms of the qualitative and quantitative data. Two points in the cumulative histogram of the quantitative measurement are used to define the linear scale of the calibration, which is then applied to the qualitative data. The cumulative histograms may be compared in the end to ensure the calibration is reasonable. The error of this calibration procedure is estimated to be $10 \%$ of the quantitative EBIC signal. The error from averaging the dark current signal is negligible compared to that of estimating the linear calibration function. The procedure is further discussed in Sec. 8.3 .

\subsubsection{Reconstruction of TEM-based EBIC profiles}

In contrast to SEM-based EBIC measurements, in TEM-based EBIC measurements with the used setup the EBIC signal is not automatically synchronized to the STEM image. In order to reconstruct an EBIC image, the synchronization was done manually based on measurement time stamps from the Keithley setup as well as the microscope software. However, the EBIC signal is inherently quantitative as it was recorded directly with an electrometer, circumventing the quantification problem described in the previous section. The Labview program VCC, which is used to record the EBIC data, in principle records current data continuously until stopped. However, the electrometer buffer can only record a maximum of 128 measurements, after which the buffer is cleared, the zero current value is recalibrated and the measurement may continue with the next step. The chosen number of measurements per step (maximum 128) is called sample number in the following. In the experiments of this work, a sample number of 20 was chosen, corresponding to a measurement time for one step of $3.4 \mathrm{~s}$. The electron beam dwell time was chosen to be a fraction of this time in order to ensure a rough synchronicity of 
scanning and EBIC recording. The detail synchronicity was achieved a posteriori using the timestamps of the VCC measurements. Each step as well as each sample within the step have unique timestamps. These are compared to the timestamps saved with the STEM image, indicating the start and stop time of the scanning process with a precision of $1 \mathrm{~s}$. These timestamps, together with the known dwell time, are used to extract the start time of recording of each STEM image pixel. The clocks of the LabView computer and the microscope computer are compared, also with a precision of $1 \mathrm{~s}$, to synchronize the EBIC and STEM timestamps. Using the given pixel size of the STEM image, the EBIC measurement timestamps can be converted to positions in a spatial profile. The comparison of the STEM and synchronized EBIC signal are shown exemplarily in Fig. Errors of the procedure depend on the chosen STEM pixel size, i.e. spatial resolution, and are about 6 pixel sizes. With the chosen total measurement times, this value increases during the scan to about 8 pixel sizes. The smallest achieved error with the highest resolution used in the experiments of this work was $0.5 \mathrm{~nm}$.

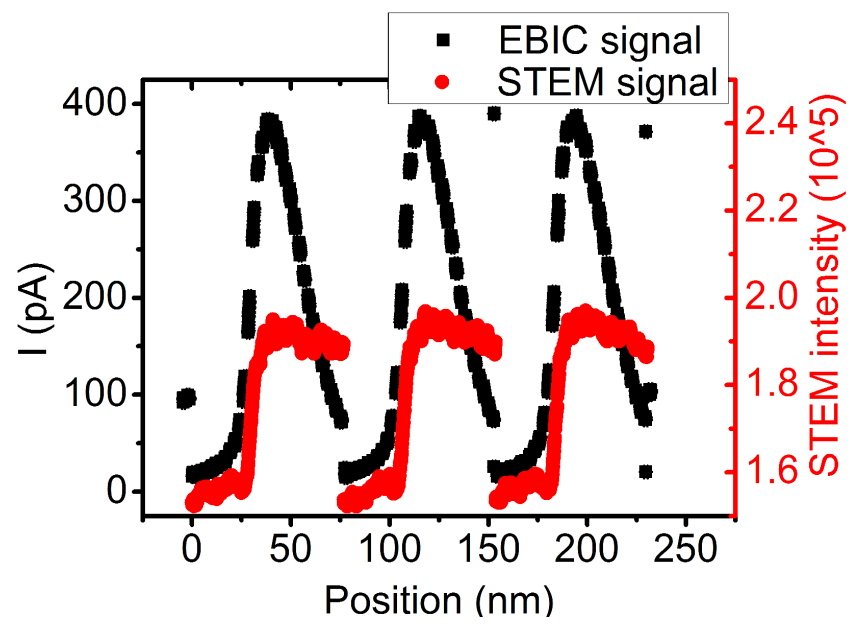

Figure 3.16: Comparison of synchronized STEM and EBIC scan lines in a TEM-based EBIC measurement 


\section{Chapter 4}

\section{Diffusion length estimation by low energy EBIC}

This chapter is a based on the publication 12

\section{Current-voltage characteristics of manganite-titanite perovskite junctions}

Benedikt Ifland, Patrick Peretzki, Birte Kressdorf, Philipp Saring, Andreas Kelling, Michael Seibt, and Christian Jooss

Published in: Beilstein Journal of Nanotechnology 2015, 6, 1467-1484; doi:10.3762/bjnano.6.152

Figures have been taken from the publication. The text has been re-written in order to focus on the contributions of the thesis author. References are collected in the bibliography of the thesis.

\subsection{Premise}

The diffusion length is an important parameter to characterize a photovoltaic device. Therefore, within an extended study of PCMO-STNO devices by current-voltage characteristics, the diffusion length was accessed by EBIC experiments.

The charge carrier collection behaviour in a p-n junction may be described by a onedimensional collection function $f(x)$ in the direction perpendicular to the $\mathrm{p}-\mathrm{n}$ junction plane, as described in Eq. 2.40. Including the effect of space charge region and diffusion length, it may be written as

$$
f_{\mathrm{SCR}+\mathrm{D}}(x)= \begin{cases}\exp \left(\frac{d_{\mathrm{PCMO}}+x}{L_{\mathrm{PCMO}}}\right), & x \leq-d_{\mathrm{PCMO}} \\ 1, & -d_{p}<x \leq d_{n} \\ \exp \left(-\frac{x-d_{\mathrm{STNO}}}{L_{\mathrm{STNO}}}\right), & x>d_{\mathrm{STNO}}\end{cases}
$$

with the space charge region size in $\mathrm{p}$ and n-doped region $d_{p}$ and $d_{n}$ as well as the diffusion length $L_{\mathrm{PCMO}}$ and $L_{\mathrm{STNO}}$, which is also dependent on the material, i.e. $L_{\mathrm{PCMO}}$ or $L_{\mathrm{STNO}}$ depending on junction side in a PCMO-STNO heterojunction. If the diffusion length is negligible, Eq. 4.1 reduces to

$$
f_{\mathrm{SCR}}(x)= \begin{cases}1, & -d_{\mathrm{PCMO}}<x \leq d_{\mathrm{STNO}} \\ 0, & \text { otherwise }\end{cases}
$$


As shown in Eq. 2.41, a cross section EBIC profile in the direction perpendicular to the p-n junction interface may be described as a convolution of the collection function and the generation function. If a simulated profile uses the collection function with negligible diffusion length, it can be compared to an experimental profile in order to find out whether a non-negligible diffusion effect is present in the p-n junction.

\subsection{Methods}

The PCMO-STNO p-n heterojunction was prepared as described in section 3.1.1. FIB preparation was used to prepare a cross section lamella in the sample, for a sketch of the geometry see Fig. 4.1(a). A large thickness of the lamella was chosen in order to include the whole electron beam generation volume. EBIC maps were collected with an electron beam with $2 \mathrm{kV}$ acceleration voltage, which provides for a small generation volume, as seen from the CASINO simulations in Fig. 4.1(b).

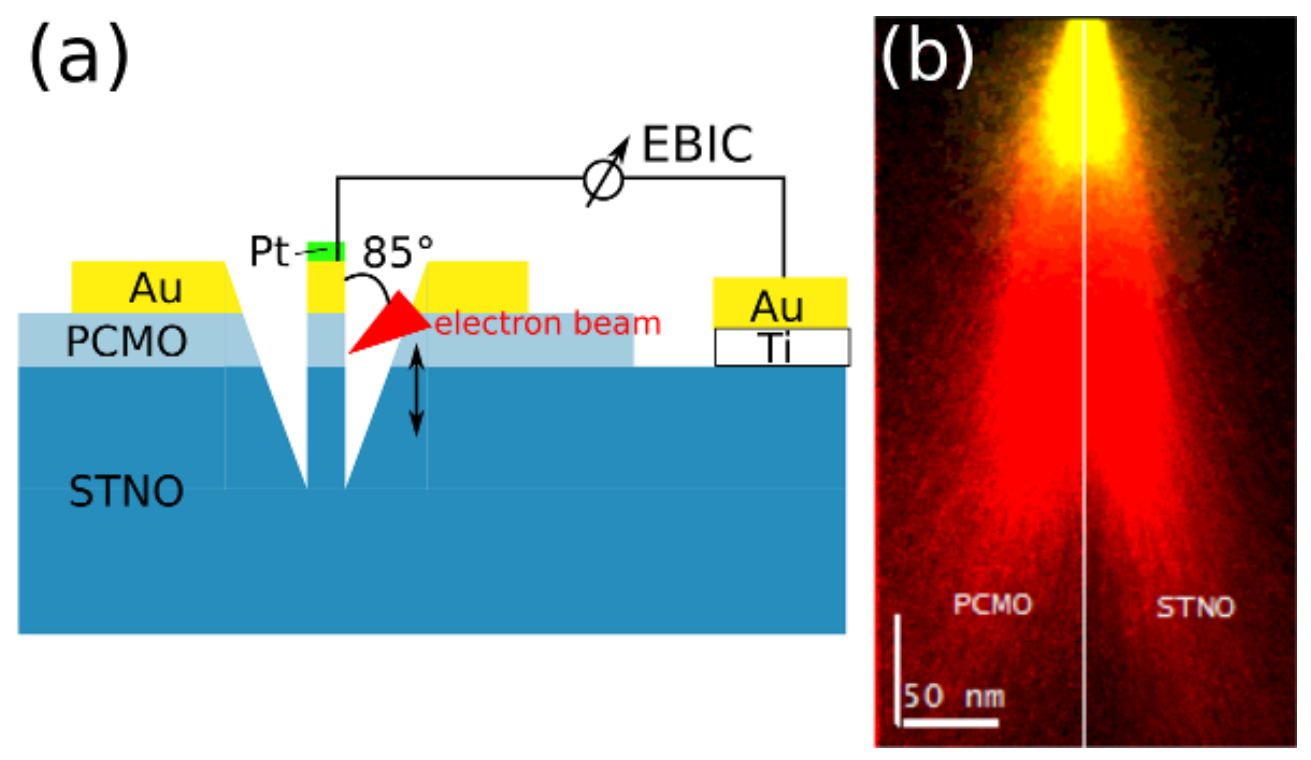

Figure 4.1: Setup of the $2 \mathrm{kV}$ cross section EBIC experiment, taken from 12. (a) sketch of the sample geometry, (b) cross section of the electron beam generation volume simulated for excitation at the PCMO-STNO interface. $2 \mathrm{kV}$ (yellow, bright) and $10 \mathrm{kV}$ (red, dark) excitation are compared, showing the generation volume in the $2 \mathrm{kV}$ case merely extends over $50-150 \mathrm{~nm}$ in all directions. The cross section lamella thickness was chosen large enough to include the whole $2 \mathrm{kV}$ generation volume.

The $2 \mathrm{kV}$ generation volume was simulated as a spatial distribution of absorbed energy in the sample by an impinging $2 \mathrm{kV}$ electron beam. The Monte Carlo software CASINO v2 was used, with $2 \cdot 10^{5}$ simulated impinging electrons and assuming an infinite cross section sample thickness. The beam was placed at the PCMO-STNO interface, and a uniform generation function for all beam positions in the simulated EBIC profile was assumed. The convolution of the simulated generation volume with $f_{\mathrm{SCR}}$ was carried out numerically, using the space charge region sizes $d_{\mathrm{PCMO}}=2.5 \mathrm{~nm}$ and $d_{\mathrm{STNO}}=27 \mathrm{~nm}$ [9]. The former is a correction of the sub-nanometer nominal value, justified from EELS experiments indicating significant $\mathrm{Mn}$ and $\mathrm{Ti}$ atom interdiffusion at the interface on the given scale. 


\subsection{Results and discussion}

Fig. 4.2 shows a cross section EBIC profile across the p-n junction interface together with the simulated profile, both normalized to their maximum. It is clearly visible that

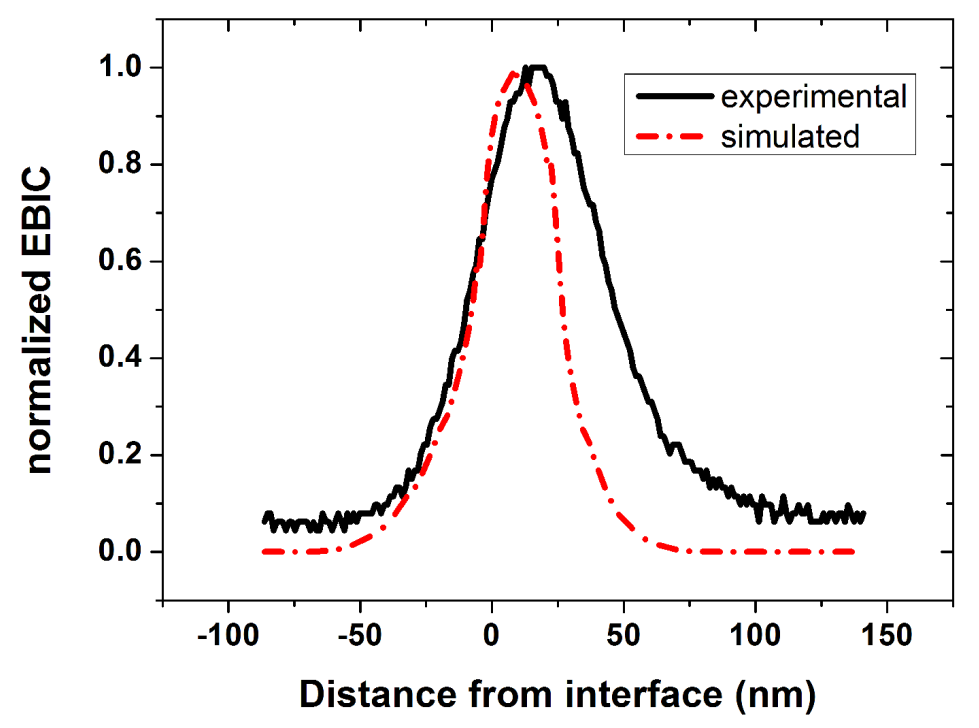

Figure 4.2: Cross section experimental and simulated EBIC profile, both normalized to their maximum, taken from [12. The experimental profile is significantly broader than the simulated one, at least on the STNO side (right). As the simulation did not include the diffusion length, a finite diffusion length can be deduced.

the experimental profile is considerably broader than the simulated one, which assumes a negligible diffusion length on both sides. At least on the STNO side, results strongly indicate a finite diffusion length. This finding can be quantified using Eq. 4.1 and 4.2 , Integrating the experimental profile $I_{\mathrm{EBIC}, \mathrm{SCR}+\mathrm{D}}(x)$ featuring a finite diffusion length and the simulated profile $I_{\mathrm{EBIC}, \mathrm{SCR}}(x)$ without a diffusion length, the convolution of generation volume and collection function simplifies to

$$
\frac{\sum I_{\mathrm{EBIC}, \mathrm{SCR}+\mathrm{D}}}{\sum I_{\mathrm{EBIC}, \mathrm{SCR}}}=1+\frac{L_{\mathrm{PCMO}}+L_{\mathrm{STNO}}}{d_{\mathrm{PCMO}}+d_{\mathrm{STNO}}},
$$

effectively cancelling out the generation volume and leaving only the terms of the collection function. For known space charge region size, this is considered a robust estimation of the sum of the diffusion lengths on both sides, as the integrated profiles provide good statistics. Applying Eq. 4.3 to the profiles shown in Fig. 4.2 leads to a combined diffusion length $L_{\mathrm{PCMO}}+L_{\mathrm{STNO}}=21.4(2) \mathrm{nm}$. The asymmetric experimental profile indicates that the majority of the combined diffusion length can be attributed to $L_{n}$, which is understandable as the charge carrier mobility is much larger in STNO than in PCMO. This simple method does not account for surface recombination, which may reduce the decay length of the experimental profile, as explained in section 2.1.4 and discussed in chapter 6. Consequently, the combined diffusion length may be treated as a lower limit. As even the combined diffusion length is in the nanometer range, using the polaron charge carrier model to explain the PCMO-STNO current-voltage characteristics [12] is justified. 


\subsection{Contributions}

PCMO-STNO p-n heterojunctions were prepared by Benedikt Ifland and Christian Jooss. FIB preparation and experiments were performed by the author, with help from Philipp Saring and Pablo Marín Perera. Simulations and analysis were performed by the author under guidance of Michael Seibt. Results were discussed by the author, Benedikt Ifland, Philipp Saring, Christian Jooss and Michael Seibt. Figures were made and text was written by the author. 


\title{
Chapter 5
}

\section{Low energy scanning transmission electron beam induced current for nanoscale characterization of $\mathrm{p}-\mathrm{n}$ junctions}

This chapter is a reproduction of the publication of the same name [23], from authors:

Patrick Peretzki, Benedikt Ifland, Christian Jooss, and Michael Seibt

Published in: Physica Status Solidi (Rapid Research Letters) 2017, 11(1), 1600358; doi:10.1002/pssr.201600358

Arrangement of figures has been modified to suit this thesis. References are collected in the bibliography of the thesis.

\begin{abstract}
Electron beam induced current (EBIC) at p-n junctions can be measured in high spatial resolution using a thin lamella geometry, where most incident electrons transmit the sample. We explore the case of low excitation energies in a wedge-shaped lamella geometry to increase resolution in a controlled way. We compare a sample with high (Si) and low (manganite-titanate heterojunction) diffusion length and use Monte Carlo based simulations as a reference. It is shown that the EBIC signal obtained from the Si junction vanishes below a thickness of $300 \mathrm{~nm}$, whereas this happens at $80 \mathrm{~nm}$ in the PCMO-STNO junction. This allows for achieving an EBIC resolution of better than $50 \mathrm{~nm}$ for the latter system. The observed fundamental differences between the silicon and the perovskites junction are discussed in terms of preparation induced 'dead' layers and surface recombination.
\end{abstract}

\subsection{Introduction}

Mapping the electron beam induced current (EBIC) is an established scanning electron microscopy (SEM) tool to analyse electrical properties of rectifying junctions [17]. The spatial resolution of EBIC is determined by two sample-dependent factors, i.e. the diffusion length of excess charge carriers and the size of the generation volume. The latter is related to multiple (inelastic) electron scattering leading to considerable beam broadening which increases with beam energy [19]. There have been several attempts to improve 
EBIC resolution into the nanometer range by using very low beam voltages [121] or by measuring EBIC in a transmission electron microscope (TEM) [122, 123 at high acceleration voltage (scanning transmission EBIC, STEBIC). In particular, Cabanel et al. have measured STEBIC at $200 \mathrm{kV}$ obtaining a resolution of about $10 \mathrm{~nm}$ [124. They used a wedge-shaped Si junction sample and measured near the space charge region down to a thickness of $\mathrm{t}=100 \mathrm{~nm}$, where carrier diffusion and beam broadening do not limit the spatial resolution. Major drawbacks of this approach are the necessity of special TEM holders including the formation of high-quality electrical contacts and the risk of introducing recombination centers due to beam damage.

In this work, we explore the approach of low energy STEBIC (LE-STEBIC) which is performed in an SEM with the focused ion beam (FIB) prepared wedge-shaped lamella left inside the sample, considerably simplifying contacting. In order to systematically study the effect of reduced beam broadening on LE-STEBIC resolution in a controlled way, we choose a wedge spanning the thickness range from zero to a thickness where the fraction of transmitted electrons vanishes, i.e. we follow the transition from LE-STEBIC to conventional EBIC. This is illustrated in Fig 5.1 which shows the absorbed energy density $\varepsilon_{a}$ for an acceleration voltage of $5 \mathrm{kV}$ as obtained from Monte Carlo simulations (CASINO v2.42 [107]); $\varepsilon_{a}$ is proportional to the number of generated electron hole pairs per incoming electron, i.e. Fig 5.1(a) visualises the generation volume. The depth profiles of $\varepsilon_{a}$ are shown in Fig 5.1(b) for two different sample thicknesses, i.e. 150nm and $500 \mathrm{~nm}$. Besides the partial transmission of electrons for $\mathrm{t}=150 \mathrm{~nm}$, the curves show a reduced contribution of backscattered electrons to $\varepsilon_{a}$ compared to $\mathrm{t}=500 \mathrm{~nm}$.
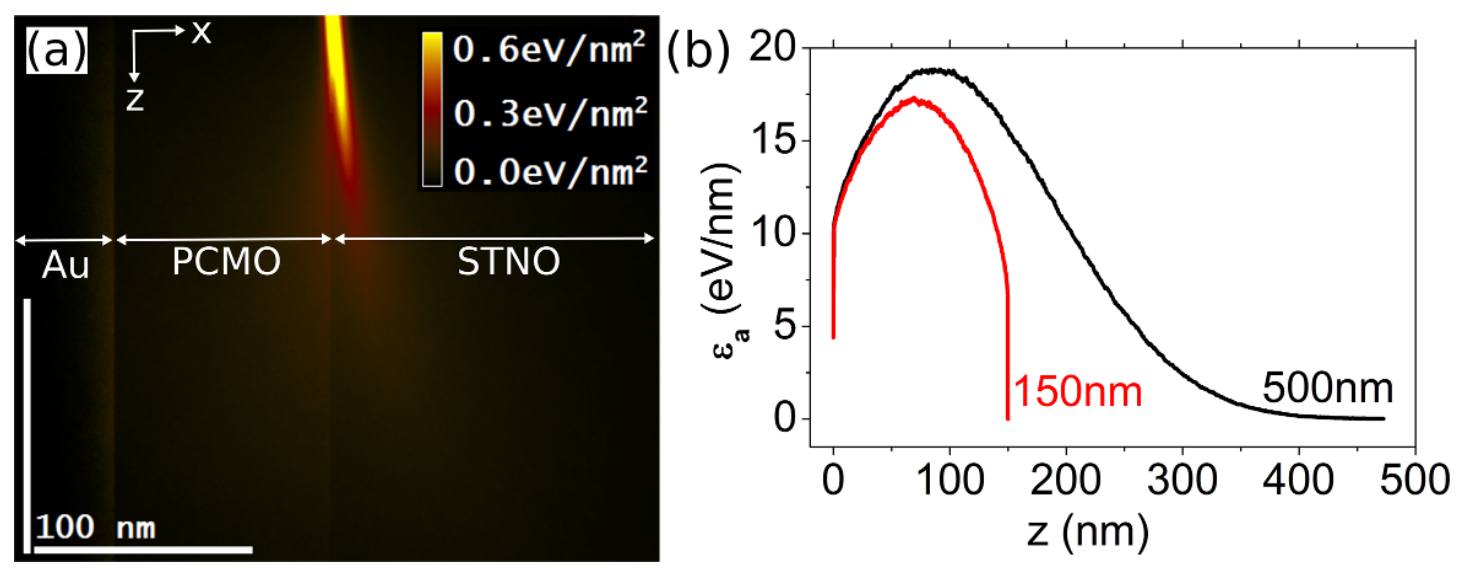

Figure 5.1: Monte-Carlo simulation (CASINO v2.42 107) of the absorbed energy density $\varepsilon_{a}$ at a PCMO-STNO p-n junction for an incident electron beam with acceleration voltage $5 \mathrm{kV}$ and a $10 \mathrm{~nm}$ beam diameter (smaller values do not drastically change $\left.\varepsilon_{a}\right)$. According to our experimental situation, simulations are performed with a $5^{\circ}$ inclination of the electron beam to the junction plane and a $\mathrm{Au}$ contact layer. (a) Energy distribution projected onto a plane perpendicular to the p-n junction plane, showing the generation volume. (b) Depth profile of absorbed energy density for $500 \mathrm{~nm}$ and $150 \mathrm{~nm}$ thicknesses. The latter shows partial electron transmission and a reduced contribution from backscattered electrons.

In order to get insight into the relevant processes, we compare two materials with vastly different recombination properties, i.e. a $\mathrm{p}-\mathrm{n}$ junction produced by $\mathrm{Al}$ alloying on n-type multicrystalline silicon and a manganite-titanite p-n heterojunction consisting of p-type $\mathrm{Pr}_{0.64} \mathrm{Ca}_{0.36} \mathrm{MnO}_{3}$ (PCMO) and n-type $\mathrm{SrTi}_{0.998} \mathrm{Nb}_{0.002} \mathrm{O}_{3}$ (STNO). Whereas the former 
has a bulk diffusion length $L_{D}$ above $50 \mu \mathrm{m}$, the latter is representative for systems with narrow bandwidths such as organic semiconductors [125] and perovskites [126]. It exhibits small polaron charge carriers because of strong electron-phonon interaction 81 and has been studied for its promising photovoltaic properties, i.e. in harvesting optical excitations at a broad wavelength range $[9,11,12$. Due to the nanosecond charge carrier lifetime in PCMO [83 and the hopping-like small conductivity, the excess carrier diffusion length is in or even below the $10 \mathrm{~nm}$ range [12]. Since both materials exhibit similar electron-matter interaction - and hence generation volumes - we experimentally realize the limiting cases of $\mathrm{L}_{D} \gg t$ and $\mathrm{L}_{D} \ll t$.

\subsection{Methods}

PCMO-STNO p-n heterojunctions have been prepared as described in detail in [12]. Briefly, a 100nm thick PCMO layer was epitaxially grown by ion beam sputter deposition on an STNO substrate, and sputtered $\mathrm{Au}$ and Ti ohmic contacts were used, as illustrated in the inset of Fig. 5.2 (a). The silicon $\mathrm{p}^{+}-\mathrm{n}$ junction was produced by $\mathrm{Al}$ alloying, by evaporating a $1 \mu \mathrm{m}$ thick $\mathrm{Al}$ layer on $\mathrm{n}$-type multicrystalline $\mathrm{Si}$ (P-concentration $10^{16} \mathrm{~cm}^{-3}$ ) and subsequently annealing at $592^{\circ} \mathrm{C}$ in an Ar atmosphere, thus forming a region with p-doping $10^{18} \mathrm{~cm}^{-3}$. Lamella preparation and EBIC measurements were carried out in an FEI Nova Nanolab 600 Dual Beam FIB microscope. (see 127 for a detailed description of the experimental setup). The resulting wedge-shaped lamella is shown in Fig. 5.2(a) and (b) as a top and side view, respectively. The thickness of the wedge at the p-n interface was determined from electron and ion images of the lamella taken from different angles in the FIB, as exemplified by Fig. 5.2. For a detailed description of the procedure, see Supporting Information.

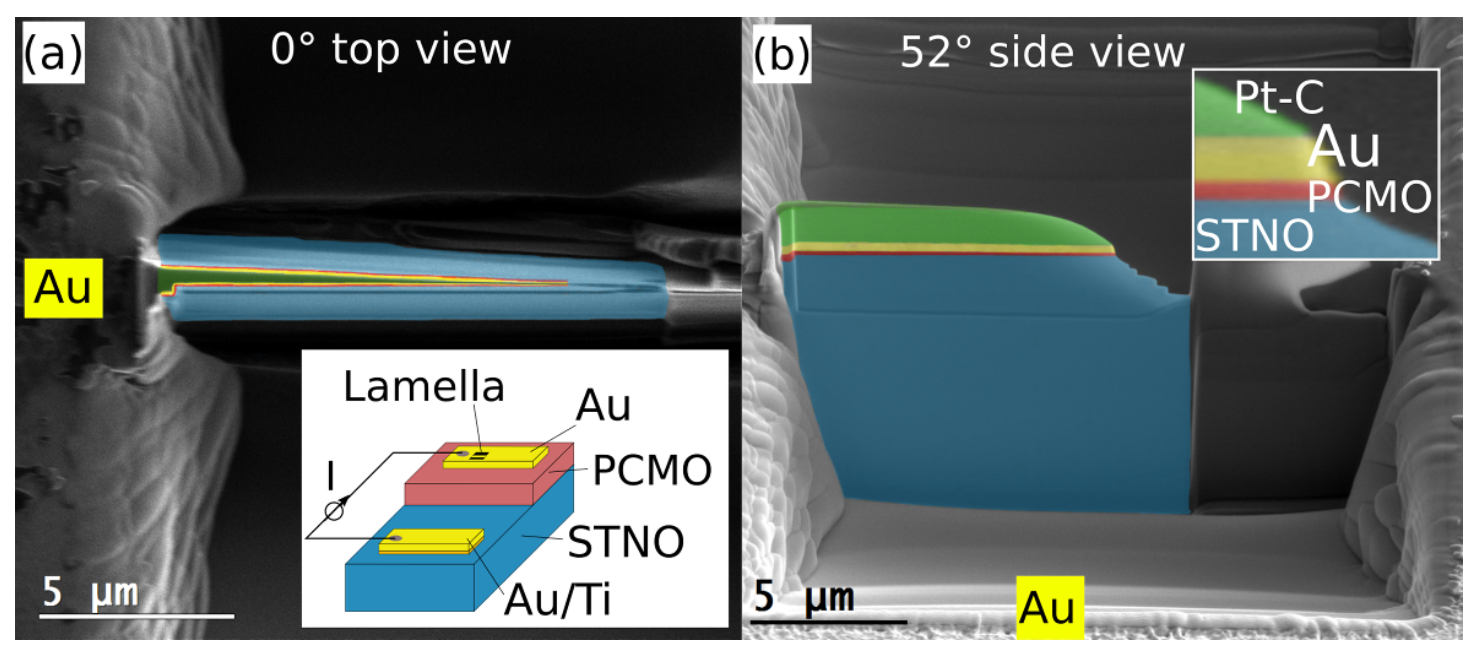

Figure 5.2: Experimental setup to measure LE-STEBIC on a PCMO-STNO p-n junction. (a) Top view of the cross-section lamella showing the prepared wedge, with inset showing the macroscopic sample geometry with contacts, (b) side view, with inset highlighting the individual sample layers (coloured).

For the measurements, the sample was tilted by $85^{\circ}$ in the SEM using a pre-tilted sample holder. EBIC images and corresponding SEM images used for the signal analysis were taken in a stitched image series across the lamella, as described in the Supporting 
Information. The missing $5^{\circ}$ to a cross-section view results in a neglibile distortion of the EBIC images, but has been accounted for in the simulations. The error of the EBIC maxima is estimated from the standard deviation of the background signal far away from the p-n junction.

\subsection{Results and Discussion}

An EBIC map of a wedge-shaped PCMO-STNO junction is shown in Fig. 5.3(a). Comparison of the SEM and EBIC signals shows that the highest EBIC signal originates from near the PCMO-STNO interface. Furthermore, it is clearly visible that the overall signal height and width decreases with decreasing lamella thickness as is shown in detail by profiles taken across the p-n junction (Fig 5.3(b) at different thicknesses indicated in the legend). This behaviour is expected from our simulations as the integral of the generation volume decreases with decreasing lamella thickness and is consistent with results obtained at 200kV STEBIC experiments [124]. Data obtained for the Si p-n junction show a qualitatively similar EBIC signal decrease with decreasing thickness (compare Fig 5.4 and the Supporting Information).
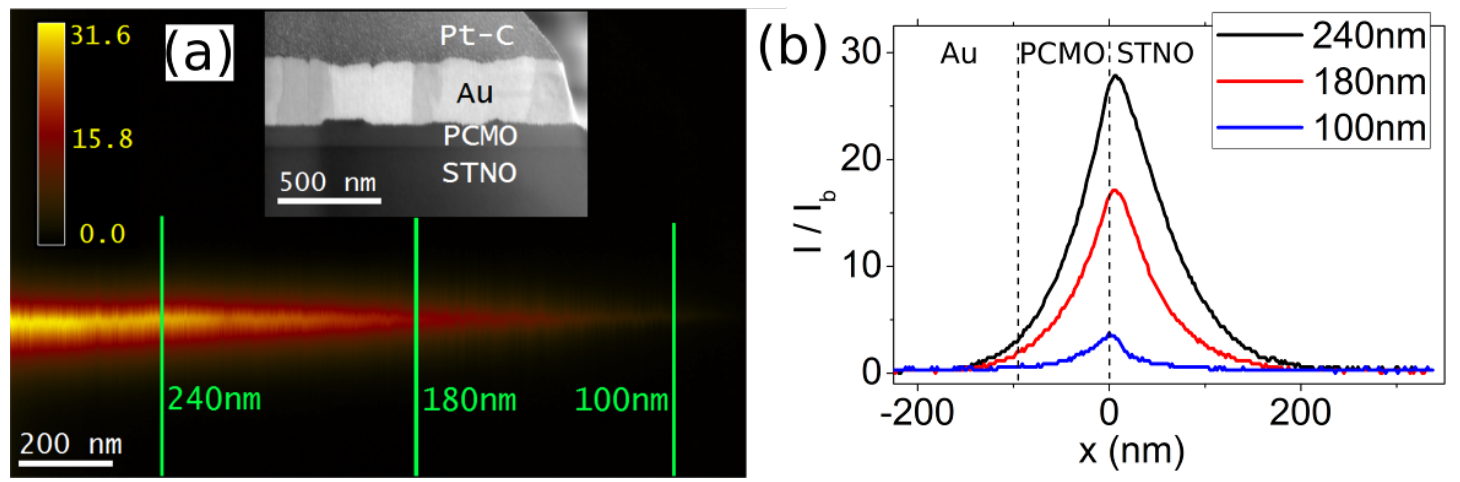

Figure 5.3: Experimental EBIC signal normalized to the incident beam current $I_{b}$ taken at the cross-section PCMO-STNO wedge at $5 \mathrm{kV}$ incident electron energy. (a) EBIC map, with inset showing the corresponding SEM image. An overall decrease of the signal from left to right, with decreasing lamella thickness, is clearly shown. (b) EBIC profiles taken at the positions marked by the green solid lines in (a), where the lamella was found to have the thickness indicated in the legend.

Further insight is gained from Fig 5.4 which shows the dependence of the maximum EBIC signal (black data points) as a function of sample thickness obtained at $5 \mathrm{kV}$ acceleration voltage for PCMO-STNO (a) and Si (b). The thickness dependence for both cases is qualitatively similar, i.e. a saturation at large thicknesses - above $t \approx 400 \mathrm{~nm}$ for PCMOSTNO and $t \approx 900 \mathrm{~nm}$ for $\mathrm{Si}$ - and a decrease with decreasing thickness which linearly extrapolates to a finite thickness of $\approx 80 \mathrm{~nm}$ for PCMO-STNO and $\approx 300 \mathrm{~nm}$ for the Si junction. More rigorously, we can define the sensitivity of the technique by estimating the noise from the standard deviation $\sigma$ of background signal measured far away from the respective junction. As a robust criterion we define $3 \sigma$ as the minimal significant EBIC signal which yields threshold thicknesses of $t_{t h}^{(P S)} \approx 80 \mathrm{~nm}$ for PCMO-STNO and $t_{t h}^{(S i)} \approx 300 \mathrm{~nm}$ for the silicon junction (compare insets in Fig 5.4 (a) and (b)), respectively, i.e. the extrapolations are consistent with the experimental sensitivity limit. A similar 
value is obtained for an acceleration voltage of $15 \mathrm{kV}$ on PCMO-STNO (see Supporting Information).
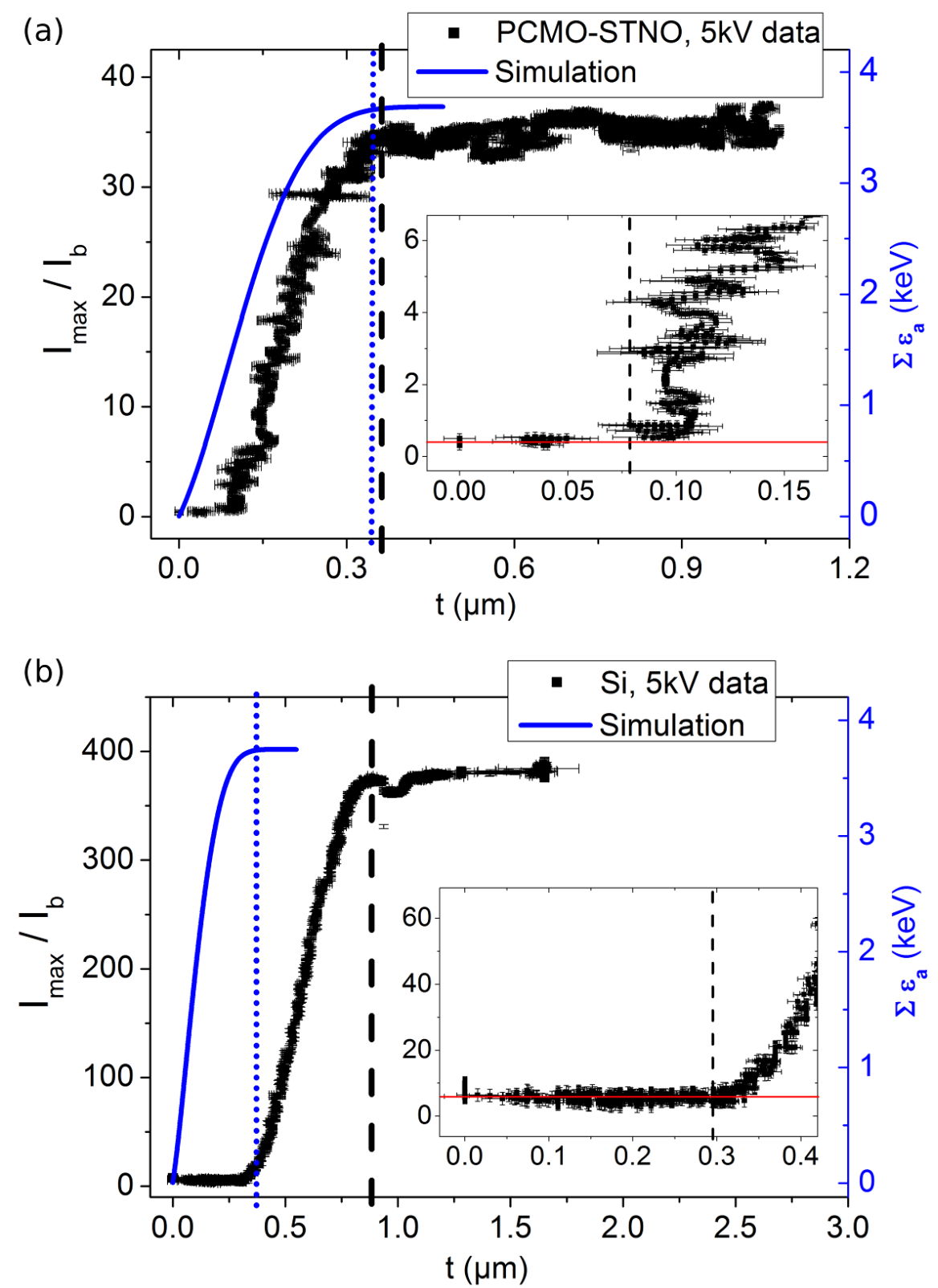

Figure 5.4: Maximum EBIC current $I_{\max }$ measured at $5 \mathrm{kV}$ versus lamella thickness $t$, normalized by the incident electron beam current $I_{b}$ (points and left axis), (a) for the PCMOSTNO and (b) for the Si lamella. The scattering of $t$ is connected to the signal-tonoise ratio of the images used for extracting the lamella thickness. Solid lines in (a) and (b) show the energy deposited in a $500 \mathrm{~nm}$ thick sample by a $5 \mathrm{kV}$ incident electron cumulated up to the given depth $t$. The insets in (a) and (b) show the threshold region with the sensitivity limit indicated as red lines. As a robust criterion, we have chosen $3 \sigma$ where $\sigma$ is the standard deviation of the EBIC signal measured far away from the respective junction. The experimental signal crosses the $3 \sigma$-level for thicknesses of $80 \mathrm{~nm}$ and $300 \mathrm{~nm}$ for PCMO-STNO and $\mathrm{Si}$, respectively, indicating the low thickness limits of LE-STEBIC measurements in the two systems; for a detailed discussion, see text. 
Despite the apparent similarity of the measured thickness dependencies for PCMO-STNO and $\mathrm{Si}$, the underlying physics is different which becomes obvious when considering the cumulative absorbed energy along the incoming beam direction as a function of sample thickness which is included as blue lines in Fig 5.4 (a) and (b). For PCMO-STNO the thickness where the EBIC signal starts to decrease below the saturation value (dashed line) roughly coincides with the thickness below which electrons are increasingly transmitted by the sample (dotted line), i.e. the decreasing EBIC signal can be attributed as being mainly due to a decreasing total generation rate as a consequence of transmitted electrons. In contrast, for the silicon p-n junction the cumulative absorbed energy along the beam already reaches its maximum at a thickness where no significant EBIC signal is detected experimentally. Hence, the decay of the signal is not related to a decreasing generation rate due to transmitted electrons, but rather to the recombination at the bottom surface which has a decreasing effect on the EBIC signal with increasing sample thickness. Using standard values for the hole mobility suitable for multicrystalline $\mathrm{Si}$ 128 we have fitted a recently proposed model for conventional cross-section EBIC 31 to our $\mathrm{Si}$ data in the saturation regime $(\mathrm{t}>800 \mathrm{~nm})$ and obtain maximum generation rates at the surface in contradiction to our experimental conditions. A tentative explanation is the existence of 'electrically damaged' or 'dead layers' at the top and bottom surfaces which do not contribute to carrier generation. Following this thought, 'surface recombination' effectively happens at the interface of the active sample region to a dead layer rather than the sample surface.

We now want to discuss the threshold thicknesses $t_{t h}$ below which no significant EBIC signal is detected. Ignoring for the moment the fundamental difference between electrostatic potentials and excess carrier currents in and near p-n junction, we may relate this threshold to dead layers which have frequently been observed and discussed in connection with off-axis electron holography experiments aiming at mapping electrostatic potentials using electron holography $[102,129]$. There, a vanishing built-in potential step is observed for a finite sample thickness typically in the several 10nm range. Such dead layers are attributed to damage produced by ion irradiation during sample preparation which can be avoided by mechanical thinning and Ar ion milling. [130. Leaving aside any charge carrier diffusion and taking into account that the top and bottom surface of the lamella have been prepared under identical conditions, an upper limit for the thickness $t_{d}$ of the dead layer can be obtained from $t_{t h}$, i.e. $2 t_{d}<t_{t h}$ which yield $t_{d}^{(P S)}<40 \mathrm{~nm}$ and $t_{d}^{(S i)}<150 \mathrm{~nm}$ for the FIB procedure employed in this work. The value of $t_{d}^{(S i)}<150 \mathrm{~nm}$ is considerably larger than the dead layer thickness of $25 \mathrm{~nm}$ deduced from off-axis electron holography of Ar ion-milled [102] and of 50nm for FIB prepared [129] Si junctions. Hence, the approach of a dead layer successfully describing electron holography data is a too rough approximation for LE-STEBIC of Si junctions. For PCMO-STNO with a threshold $t_{t h}^{(P S)}=80 \mathrm{~nm}$ smaller than $t_{t h}^{(S i)}$, but still larger than the combined PCMO-STNO diffusion length of $\mathrm{L}_{D}^{(P C M O)}+\mathrm{L}_{D}^{(S T N O)}=21 \mathrm{~nm}$ 12, the effect of surface recombination should be less pronounced. For a rough estimate, it can be assumed that surface recombination considerably affects the injection level in the material once the sample thickness is comparable or smaller than the diffusion length which is the case for the Si junction for all thicknesses, whereas for PCMO-STNO the condition is met close to $t_{t h}^{(P S)}$. Hence, for the systems studied here we have to conclude that both, dead layers and surface (interface) recombination have to be taken into account in a quantitative model.

Finally, we want to discuss the spatial resolution of LE-STEBIC which is ultimately 
limited by the lateral extension of the generation volume at the respective threshold thickness. As a criterion we use the full-width-at-half-maximum (FWHM) of the lateral extension as calculated by CASINO which for $\mathrm{Si}$ at $5 \mathrm{kV}$ yields $\mathrm{FWHM} \approx 250 \mathrm{~nm}$. It should be noted, that due to the somewhat large value of $\mathrm{t}_{t h}^{(S i)} \approx 300 \mathrm{~nm}$ the fraction of transmitted electrons is negligible, i.e. LE-STEBIC in this case reduces to conventional EBIC. In contrast, for PCMO-STNO we obtain FWHM $\approx 50 \mathrm{~nm}$ which corresponds to the width of profiles taken at thicknesses just above $\mathrm{t}_{t h}^{(P S)} \approx 80 \mathrm{~nm}$. A slight increase of the acceleration voltage to e.g. $15 \mathrm{kV}$ and a smaller beam diameter reduce the FWHM to $10 \mathrm{~nm}$ for PCMO, which matches the resolution obtained in STEBIC experiments at $200 \mathrm{kV}$ [124.

\subsection{Conclusion}

In summary, the decribed LE-STEBIC approach allows increasing the spatial resolution by dramatically reducing the generation volume in thin samples at $5 \mathrm{kV}$ beam energy. Hence, high resolution SEM-based experiments are possible with a FIB prepared lamella left in the sample for easy contacting. Experimental data from wedge-shaped lamellas from materials exhibiting a high ( $\mathrm{Si}$ ) or low (PCMO-STNO) diffusion length show a threshold for the sample thickness below which not significant signal is detected. This is attributed to the effect of FIB preparation inherent surface damage resulting in 'dead' layers which are thinner for the PCMO-STNO systems facilitating a higher possible EBIC resolution below 50nm. Hence, LE-STEBIC is especially useful for short diffusion length systems, i.e. for thin film photovoltaic systems and nanostructures.

\subsection{Acknowledgement}

We thank Tobias Meyer for a sketch in Supporting Information, Hendrik Spende for preparing the Si sample, and Arne Ahrens for valuable discussion. Funding from the Deutsche Forschungsgemeinschaft (DFG) under Grant No. SFB1073, project B02 is gratefully acknowledged. 


\subsection{Supporting Information}

\subsubsection{Determination of the wedge lamella thickness gradient}

The thickness of the wedge lamella at the p-n interface was determined from electron and ion images taken in the FIB from a top and a side view, see. Fig. 1 in main article for an example. From the preparation method, we assume the lamellas to have a double wedge shape with a different wedge angle in lateral and depth direction, as illustrated in Fig. 5.5. The lateral and depth wedges were adressed in a different way, as described in the following.

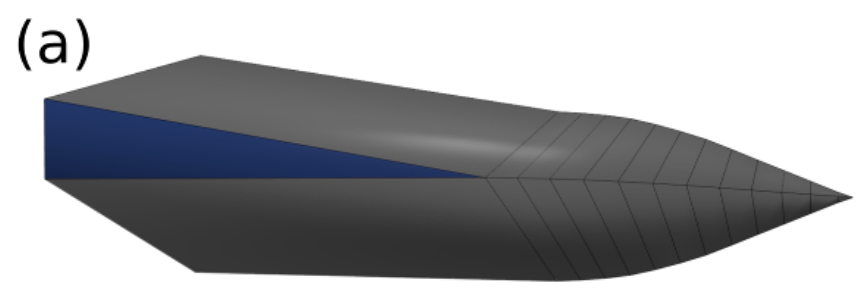

\section{(b)}

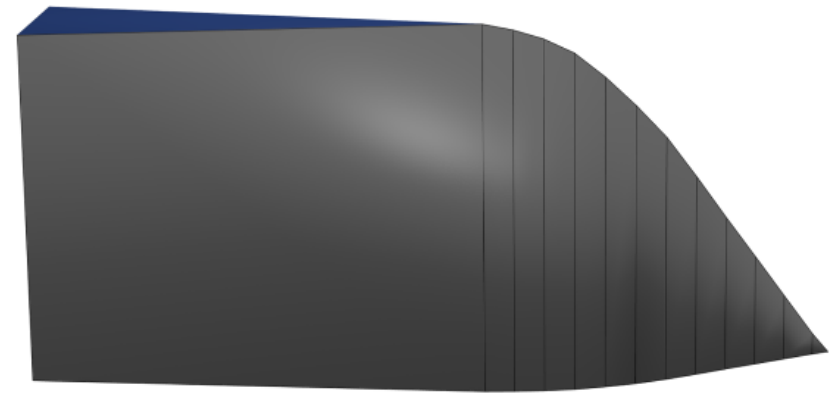

Figure 5.5: Scheme of the double wedge structure assumed for the prepared lamellas, seen from (a) top and (b) side.

The distance between high-contrast interfaces in the images was automatically determined from the distance of intensity jump thresholds in profiles along the wedge. The thresholds were locally adjusted in order to take into account gradual intensity changes or local roughness of the wedge. Using this procedure, the lateral wedge was directly determined from a top view image which shows the thickness gradient at the lamella surface (Surface thickness in Fig. 5.6). The depth wedge angle was estimated by comparing features in corresponding top and side view images, using the known tilt angle between the images and trigonometry. It is fairly small for all lamellas, between 0 and 5 degrees. Furthermore, the distance from the lamella surface to the high contrast metal contact p-layer interface (Au-PCMO and $\mathrm{Al}-\mathrm{Si}$ ) was determined on side view images using intensity jump thresholds. By taking into account the estimated depth wedge angle, the additional thickness at the p-n interface was calculated from this distance (Additional thickness in Fig. 5.6). As the p-n interface is not visible in the Si lamella, we chose to ignore the p-layer thickness in this procedure. The thickness is only $100 \mathrm{~nm}$ and about $50 \mathrm{~nm}$ for PCMO and p-Si, respectively, so the error is negligibly small in the thickness projection. 
Although the same FIB preparation procedure was used, the three used wedges in this work (two in the PCMO-STNO and one in Si) turned out to differ considerably. So each double wedge had to be determined independently. In particular, the top view image of the $\mathrm{Si}$ wedge revealed its depth wedge angle is approximately zero, i.e. the lamella sides are not visible from this view angle. Thus, the additional thickness was neglected in this case. For one of the PCMO-STNO lamellas, a depth wedge was only visible on one side. Figure 5.6 shows the determined thickness gradient together with the EBIC maximum data for this wedge, which was used for the $5 \mathrm{kV}$ EBIC measurement.

For the analysis of the EBIC maximum behaviour along the wedge, single images at a resolution of about $2 \mathrm{~nm}$ per pixel were taken. The SEM images were stitched together and drift corrected by comparing smooth interfaces and local features in the metal contact layer. By comparing the stitched images to overview images of a lower resolution, residual astigmatism could be determined and corrected by warping the images. The same correction was always applied to the stitched EBIC images, as EBIC and SEM images were recorded simultaneously. The extracted EBIC maxima from the stitched images are shown in Fig. 5.6 for the $5 \mathrm{kV}$ measurement on the PCMO-STNO lamella.

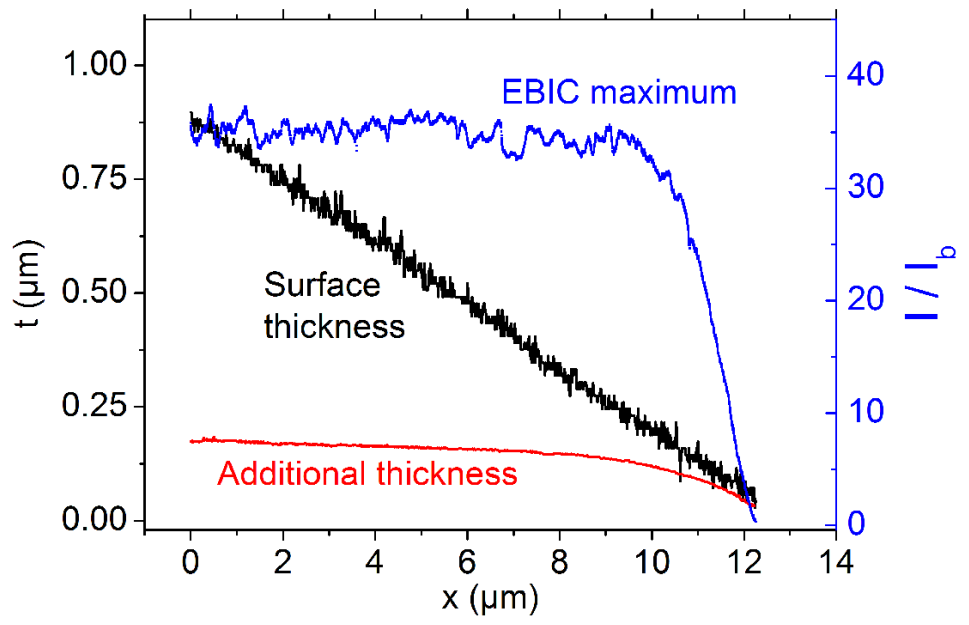

Figure 5.6: Thickness gradient determined for the PCMO-STNO wedge lamella, which was used for the $5 \mathrm{kV}$ EBIC measurements. The surface thickness was determined from the lateral wedge in a top view image, whereas the additional thickness at the p-n interface because of the one-sided depth wedge was calculated from a side view image. The total thickness is the sum of surface and additional thickness at each position. The thickness results were linearly interpolated in order to be compared to the EBIC maximum data.

\subsubsection{EBIC map of the Si wedge}

Fig. 5.7 shows the EBIC map recorded from the prepared $\mathrm{Si}$ wedge lamella at $5 \mathrm{kV}$ incident electron energy. The noticeable kink in the EBIC signal results from a grain boundary located approximately in the center of the Si lamella. As for this sample the pre-tilt of $45^{\circ}$ could not be used, the measurements were carried out under an angle of $52^{\circ}$ from the beam to the sample surface. The resulting warp of the sample geometry has been corrected in the line profiles. The maxima in the line profiles, as exemplarily shown 
in (b) were plotted versus the local wedge thickness in Fig. 4(b) in the main article. Note that two different background EBIC levels exist in this junction in the $\mathrm{Al}$ and $\mathrm{Si}$ region. As we are primarily interested in the Si p-n junction, we use the background signal on the $\mathrm{Si}$ side as a reference to subtract from the signal maximum.
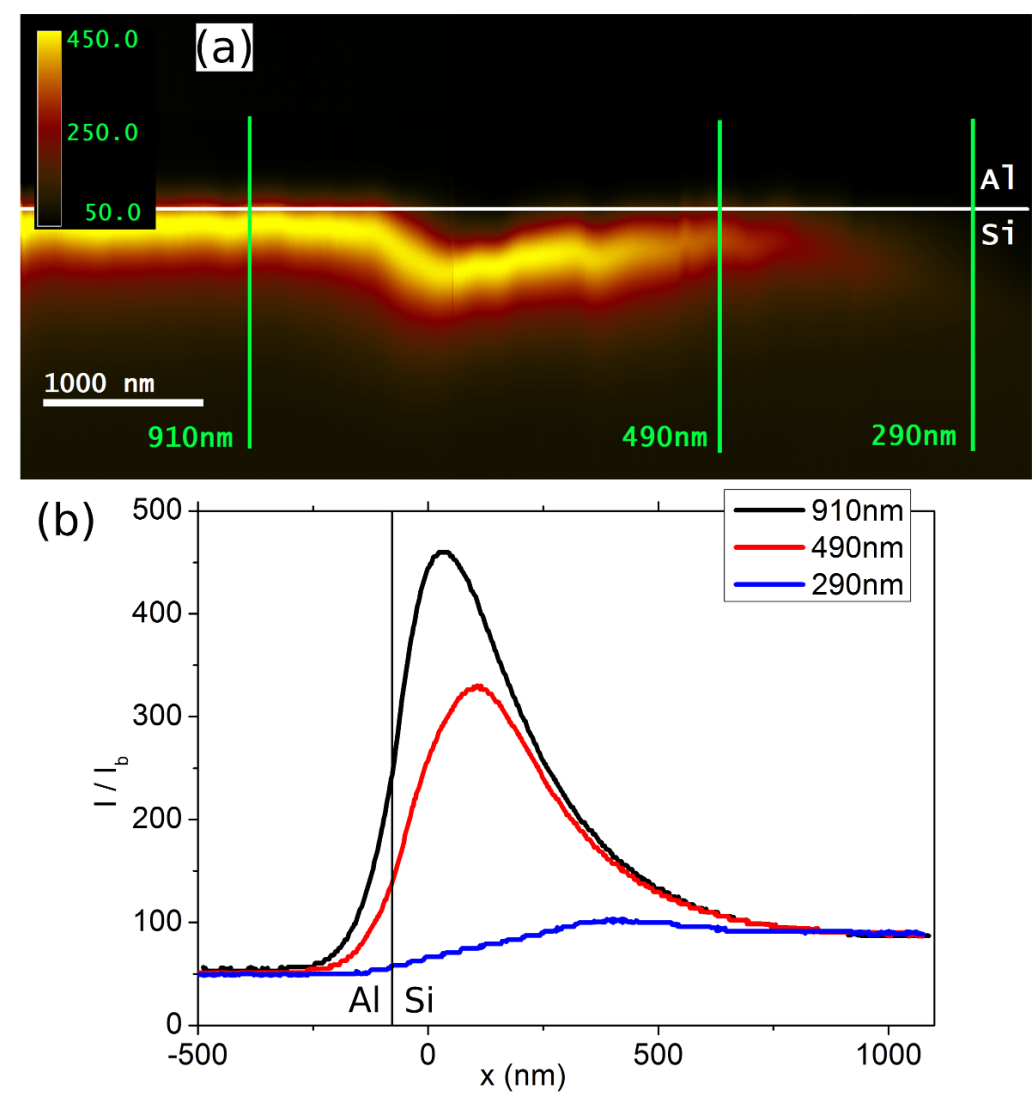

Figure 5.7: Experimental EBIC signal normalized to the incident beam current taken at the crosssection Si wedge at $5 \mathrm{kV}$ incident electron energy. (a) EBIC map. An overall decrease of the signal from left to right, with decreasing lamella thickness, is clearly shown. (b) EBIC profiles taken at the positions marked by the green solid lines in (a), where the lamella was found to have the thickness indicated in the legend. A clear signal decrease with decreasing thickness is visible, approaching two different background levels for $\mathrm{Al}$ and $\mathrm{Si}$. The kink in the EBIC signal results from the presence of a grain boundary in the mc-Si.

\subsubsection{PCMO-STNO EBIC maxima at $15 \mathrm{kV}$}

Results from a scan of the PCMO-STNO sample with $15 \mathrm{kV}$ acceleration voltage are shown in Fig. 5.8. In contrast to the $5 \mathrm{kV}$ results in Fig. 4 in the main article, a saturation of the EBIC maximum for large sample thickness is not reached. As the simulation of the cumulative absorbed energy shows, the used wedge lamella is simply not thick enough to show a saturation. The EBIC maximum is below the chosen $3 \sigma$ background threshold (see main article) for thicknesses smaller than $70 \mathrm{~nm}$, which is close to the $80 \mathrm{~nm}$ threshold obtained from the $5 \mathrm{kV}$ measurement. 

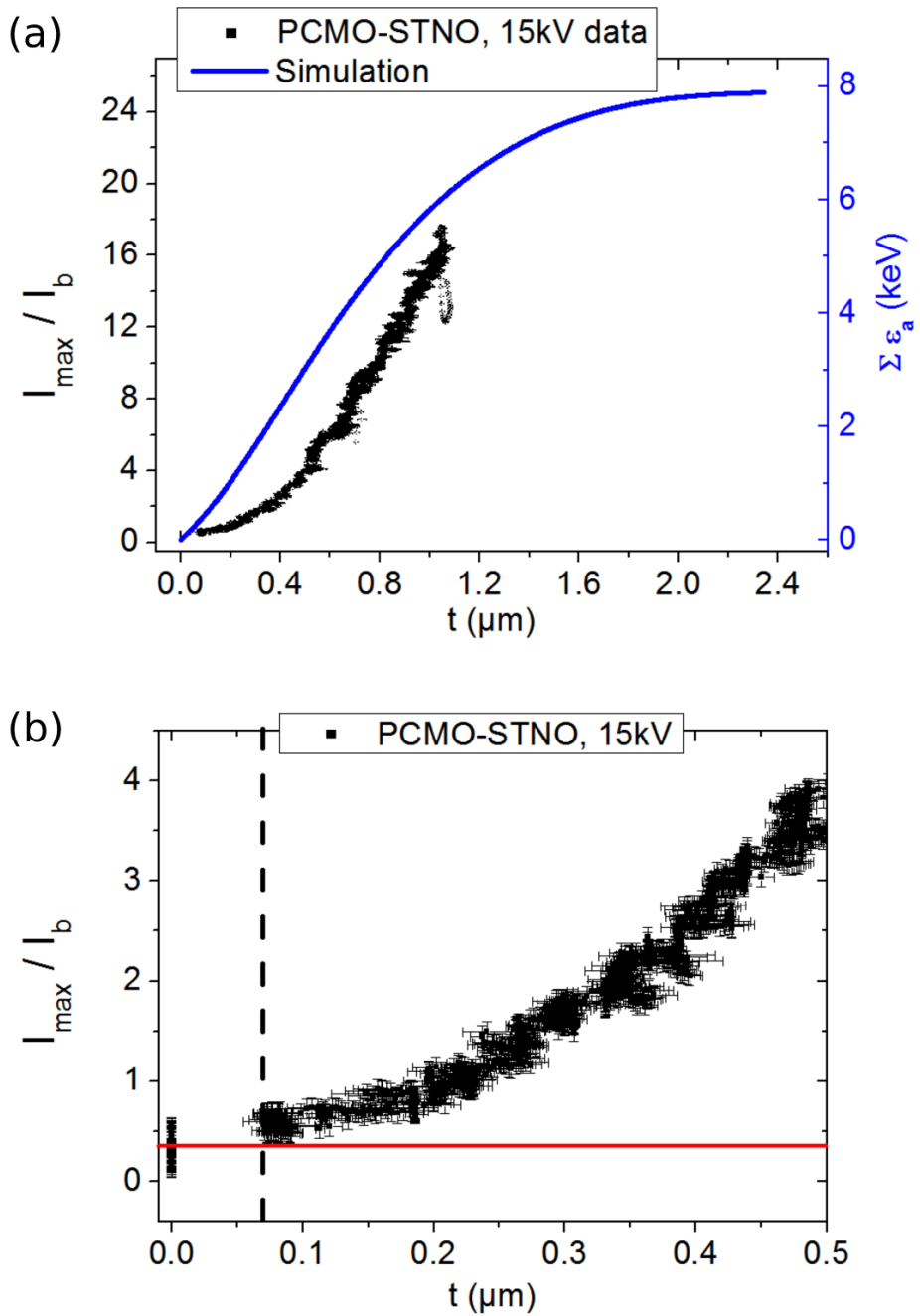

Figure 5.8: (a) Maximum EBIC current $\mathrm{I}_{\max }$ measured at $5 \mathrm{kV}$ versus lamella thickness t, normalized by the incident electron beam current $\mathrm{I}_{\mathrm{b}}$ (points and left axis). The solid line shows the energy deposited in a $500 \mathrm{~nm}$ thick sample by a $5 \mathrm{kV}$ incident electron cumulated up to the given depth t. EBIC clearly does not saturate up to the maximum lamella thickness. (b) shows the threshold region with the sensitivity limit indicated as red lines. As a robust criterion, we have chosen $3 \sigma$ where $\sigma$ is the standard deviation of the EBIC signal measured far away from the respective junction. The experimental signal crosses the $3 \sigma$ level for a thickness of $70 \mathrm{~nm}$, which is close to the $80 \mathrm{~nm}$ threshold obtained for the $5 \mathrm{kV}$ results.

\subsection{Contributions}

PCMO-STNO p-n heterojunctions were prepared by Benedikt Ifland and Christian Jooss. Hendrik Spende prepared the Si $\mathrm{p}^{+}-n$ junction. FIB preparation, experiments, simulations and analysis were performed by the author under guidance of Michael Seibt. Results were discussed by all authors and Arne Ahrens. Fig. 5.5 was contributed by Tobias Meyer, all other figures were made by the author. Text was primarily written by the author, with contributions by Michael Seibt, Christian Jooss and Benedikt Ifland. 



\section{Chapter 6}

\section{Nanometer range diffusion length in a polaronic perovskite solar cell measured by scanning transmission EBIC}

Patrick Peretzki, Tobias Meyer, Jonas Lindner, Benedikt Ifland, Christian Jooss, and Michael Seibt

\subsection{Abstract}

Third generation photovoltaic systems frequently feature small excess charge carrier diffusion lengths down to the nanometer range. For successful research and development such quantities have to be reliably measured. We show that Electron Beam Induced Current (EBIC) in scanning transmission mode (STEBIC) provides quasi-point-source conditions in a cross-section geometry. We demonstrate STEBIC resolution in the nanometer range for a manganite-based p-n heterojunction with polaronic excitations. The spatial width of STEBIC profiles obtained at 80 and $300 \mathrm{kV}$ is identical within experimental error which provides evidence that a reliable and robust diffusion length determination has been realized. As a novel approach we combine STEBIC and electron energy loss spectrum imaging thus facilitating to accurately measure the shift of the maximum STEBIC signal from the chemical junction towards the lower doped side of the heterojunction. Furthermore, we discuss the remaining effect of TEM lamella surface recombination and dead layers and how it can be controlled experimentally.

\subsection{Introduction}

Third generation photovoltaics 131] aims at conversion efficiencies beyond the ShockleyQueisser limit of single-junction solar cells [1]. Besides the somewhat classical approach of tandem or multi-junction solar cells numerous concepts have been proposed and are currently studied in detail. The latter include organic materials, quantum dots, hybrid and perovskite materials $125,126,132,133$. An important benchmark of classical silicon solar cells is the excess carrier lifetime which in those systems is on the order of $1 \mu \mathrm{m}$ or below which requires new approaches for characterization and modeling [134.

In this work, we focus on an anorganic perovskite manganite-titanite heterostructure with a hole conducting $\mathrm{Pr}_{1-x} \mathrm{Ca}_{x} \mathrm{MnO}_{3}$ (PCMO) epitaxially grown on n-doped $\mathrm{SrTi}_{1-y} \mathrm{Nb}_{y} \mathrm{O}_{3}$ (STNO), which are known to form very stable p-n heterojunctions 11. Due to strong 
electron-phonon coupling, electric conductivity in PCMO results from charge carriers of small polaron type [87, 135. Optical excitations of PCMO with doping $x=0.35$ have a lifetime in the nanosecond regime at low temperatures [13], while excitations over the $3.2 \mathrm{eV}$ STNO bandgap for $y \approx 0.002$ exhibit a lifetime of around $10 \mathrm{~ns}$ [136. A further prominent feature is the large spectral range of absorbed external optical radiation spanning from the near-infrared to the ultraviolet range, which is attributed to polaronic charge carriers 15. Hence, these heterojunctions provide the possibility to harvest broadband optical excitations and indicating their promising potential in photovoltaics [9, 12, 14]. The hopping-like polaron transport is associated with a small mobility of $0.02 \frac{\mathrm{cm}^{2}}{\mathrm{~V} \cdot \mathrm{s}}$ at room temperature which, using the Einstein relation, yields a excess charge carrier diffusion length of around $7 \mathrm{~nm}$ for PCMO (subsequently referred to as 'diffusion length', for simplicity). Due to larger charge carrier mobility $\left(1 \frac{\mathrm{cm}^{2}}{\mathrm{~V} \cdot \mathrm{s}}\right)$, a higher diffusion length of $160 \mathrm{~nm}$ is expected for STNO. High doping levels of PCMO and STNO furthermore lead to space charge regions estimated in the $1-10 \mathrm{~nm}$ range [9] indicating all relevant quantitities to be on the nanometer scale.

Electron beam induced current (EBIC) is a well-established technique to measure diffusion lengths in the micron and sub-micron range (for review, see Ref. 17]). Besides the classical plan view approach, cross section or 'normal collector' geometry provides a particularly direct method for measuring the diffusion length: excess carrier generation by a 'point-like' electron beam results in EBIC profiles in the field-free region outside the charge-separating junction which decay exponentially with the decay constant being the diffusion length [17]. The validity of this simple picture requires that the diffusion length is considerably larger than the junction width and, in particular, the spatial extension of the sample volume acting as a source of excess electrons and holes typically denoted as the generation volume. The latter is ultimately limited by electron (multiple) scattering inside the material, i.e. by the beam voltage and the sample thickness. In addition, surface recombination has to be taken into account 41] and preparation-induced 'dead' layers at sample surfaces which have been shown for electron holography [102, 129] and recently also for EBIC [23].

If EBIC resolution below about $100 \mathrm{~nm}$ is required it is unavoidable to measure under conditions where a substantial part of incident electrons are transmitted, i.e. beam broadening from multiple electron scattering is prevented. Such scanning transmission EBIC (STEBIC) can still be performed in a scanning electron microscope, where a spatial resolution of $50 \mathrm{~nm}$ can be achieved which we described as low energy STEBIC [23]. There we demonstrated the beneficial effect of a wedge-shaped sample geometry which allows for adjusting the sample thickness to the required spatial resolution and at least qualitatively controlling the effect of dead layers. Still higher STEBIC resolution has been obtained at higher beam voltages $[124,137,140]$ although the reliable measurement of diffusion lengths below $10 \mathrm{~nm}$ has not been realized so far.

In this work, we perform STEBIC studies in STEM mode at beam voltages of 80 and $300 \mathrm{kV}$ of PCMO-STNO heterojunctions. Excess carrier diffusion lengths of $L=3 \mathrm{~nm}$ for PCMO and $L=17 \mathrm{~nm}$ for STNO are extracted from STEBIC profiles across the junction. In order to arrive at quantitative results, we use a wedge shape cross section sample geometry comparable to that reported previously [23]. Combining STEBIC with electron energy loss spectroscopy (EELS) 96 enables us to (i) tightly control local specimen thickness which is a pre-requisite to extract the diffusion length from raw data and (ii) measure the STEBIC profile with respect to the chemical junction with a high accu- 
racy. The observed thickness-dependent shift of the maximum STEBIC signal (6-10 nm) is discussed in terms of a thickness-dependent injection level. Finally, we discuss our results in the light charge order in polaronic PCMO on photovoltaic energy conversion.

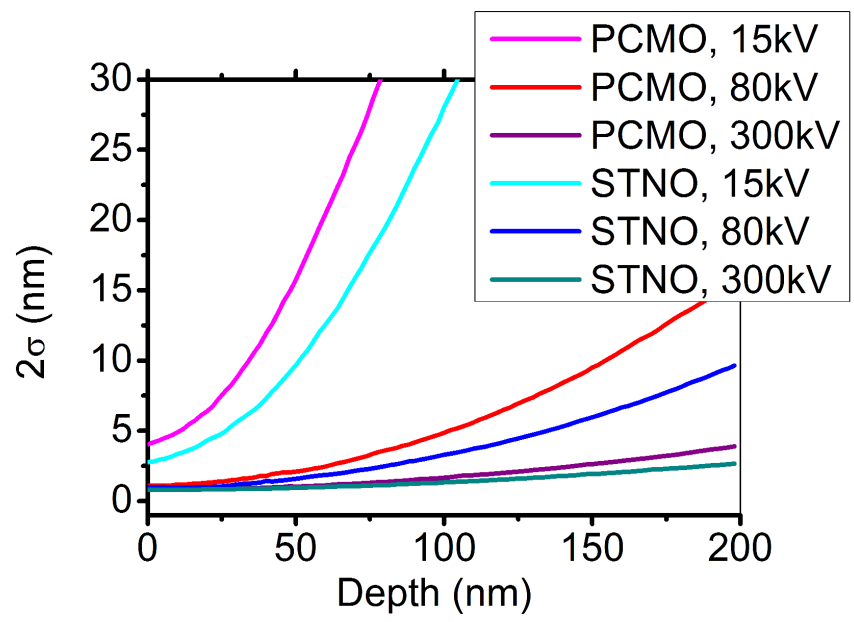

Figure 6.1: Lateral $2 \sigma$ range of the deposited energy distribution in a PCMO sample for three electron beam acceleration voltages. The distribution was simulated by the Monte Carlo program CASINO. The greatly reduced sample depth dependence of the $80 \mathrm{kV}$ and $300 \mathrm{kV}$ (STEM) situation in comparison to $15 \mathrm{kV}$ (SEM) shows that STEM is more suitable for high-resolution STEBIC measurements than SEM.

In order to illustrate our experimental approach and its analysis, we first discuss STEBIC spatial resolution requirements to reliably measure the diffusion length. There are at least three partly counteracting aspects: (i) the lateral extension $w_{g}$ of the generation volume should be smaller than the diffusion length in order to avoid extensive deconvolution procedures which are frequently associated with large uncertainties. In additon, (ii) the TEM lamella thickness should be considerably larger than the diffusion length in order to minimize the effect of surface recombination, and (iii) the minimal lamella thickness is determined by the signal-to-noise ratio of the setup.

For considering (i) and (ii) let us for the moment ignore the effect of carrier diffusion, i.e. assume the spatial resolution to be limited by $w_{g}$. Fig. 6.1 summarizes Monte Carlo simulations using CASINO 3.3 developed by Demers et al. [108], which is optimized to include electron beam acceleration voltages above $30 \mathrm{kV}$. As parameters, we chose $1 \mathrm{~nm}$ beam diameter and 200000 simulated electrons. The spatial energy distribution was calculated with $0.5 \mathrm{~nm}$ lateral and $2 \mathrm{~nm}$ depth spatial resolution. The Joy-Luo model [110] was chosen for energy loss calculation. The resulting three-dimensional energy distribution is averaged laterally in order to provide radial and depth information. Finally, $w_{g}$ is estimated as $2 \sigma$ of the azimuthally integrated deposited energy. From Fig. 6.1 it is immediately evident that SEM based EBIC is not suitable for the requirements of our system. Acceleration voltages of 80 and $300 \mathrm{kV}$ with STEM lamella thicknesses around 100-200 $\mathrm{nm}$ provide sufficiently small $w_{g}$ to ensure that the spatial resolution of STEBIC in PCMO-STNO is only limited by the diffusion length. We further fulfill condition (ii) by measuring STEBIC for various sample thicknesses using a wedge shape sample geometry adopted from [23] as described in detail below. We note here, that condition (iii) is met for thicknesses above $50 \mathrm{~nm}$ which is roughly the dead layer thickness for our samples. 


\subsection{Results and Discussion}

We first describe typical properties of STEBIC data measured in STEM mode and simultaneously obtained EELS data. The recorded cross section STEBIC profiles in SEM $(15 \mathrm{kV})$ and STEM $(80$ and $300 \mathrm{kV})$ have a general shape as shown in Fig. 6.2. The chemical interface position and the local lamella thickness were determined by in-situ low loss EELS measurements, as described in detail in the methods section. Compared to $15 \mathrm{kV}$ from SEM, the 80 and $300 \mathrm{kV}$ STEBIC profiles from STEM are much more narrow, directly showing the resolution advantage in STEM. Note that the 80 and $300 \mathrm{kV}$ profiles are coinciding, confirming the finding from the simulation in Fig. 6.1 that the role of the generation volume is dramatically decreased in the TEM measurements compared to SEM.

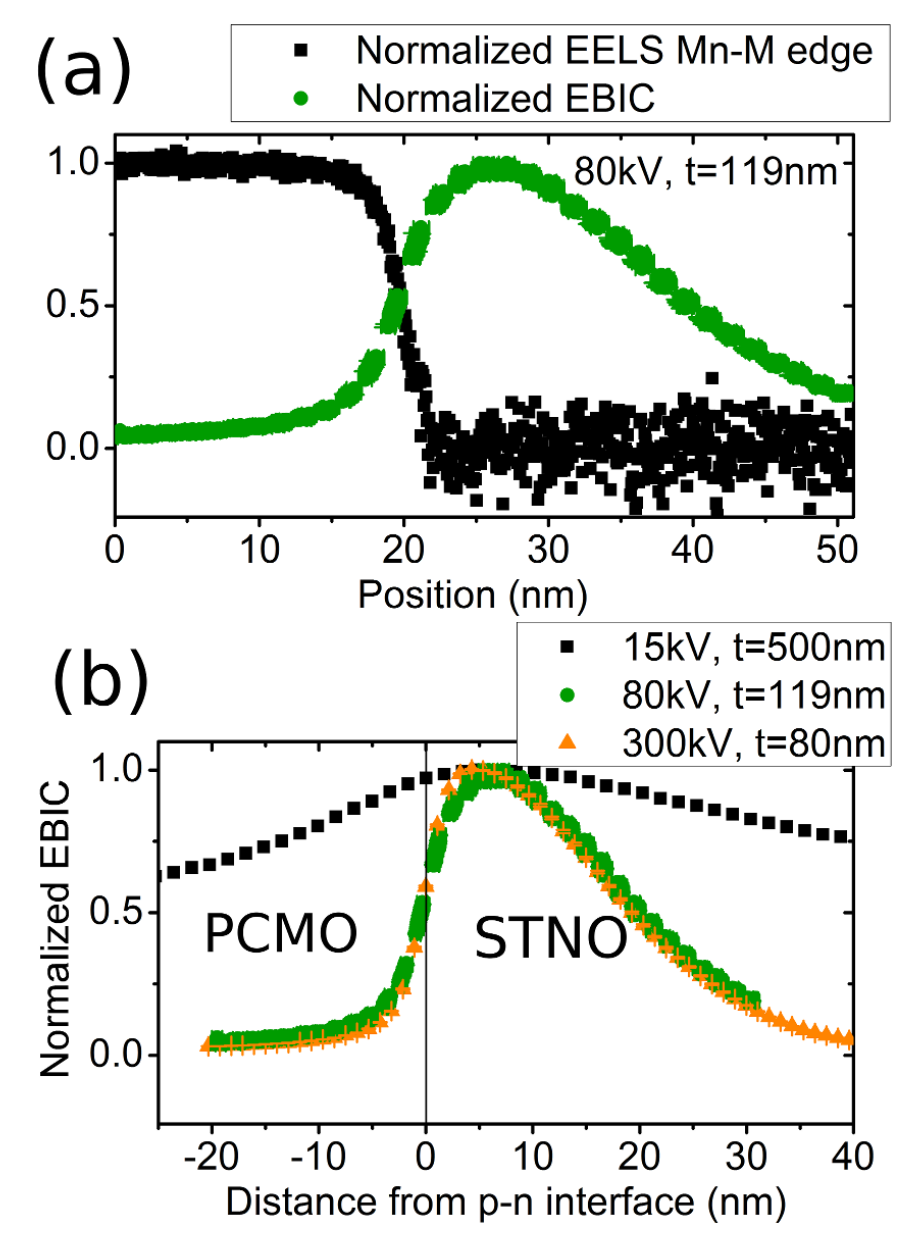

Figure 6.2: (a) EELS Mn-M edge profile across the PCMO-STNO interface, which is used to determine the interface position in the STEBIC profile in TEM measurements; (b) STEBIC profiles for three electron beam acceleration voltages, showing the much narrower signal in the TEM $(80,300 \mathrm{kV})$ than in the SEM $(15 \mathrm{kV})$ situation. The coinciding profiles for 80 and $300 \mathrm{kV}$ show that the profile shape is not significantly dependent on the electron beam generation volume in the TEM.

$80 \mathrm{kV}$ STEBIC profiles, with the local sample thickness as a parameter, were analyzed in three different aspects, (i) their decay length, (ii) the signal height of the profile maximum, and (iii) the distance of the maximum from the determined chemical interface 
between PCMO and STNO. These aspects are addressed consecutively in the following.

\subsubsection{Decay length}
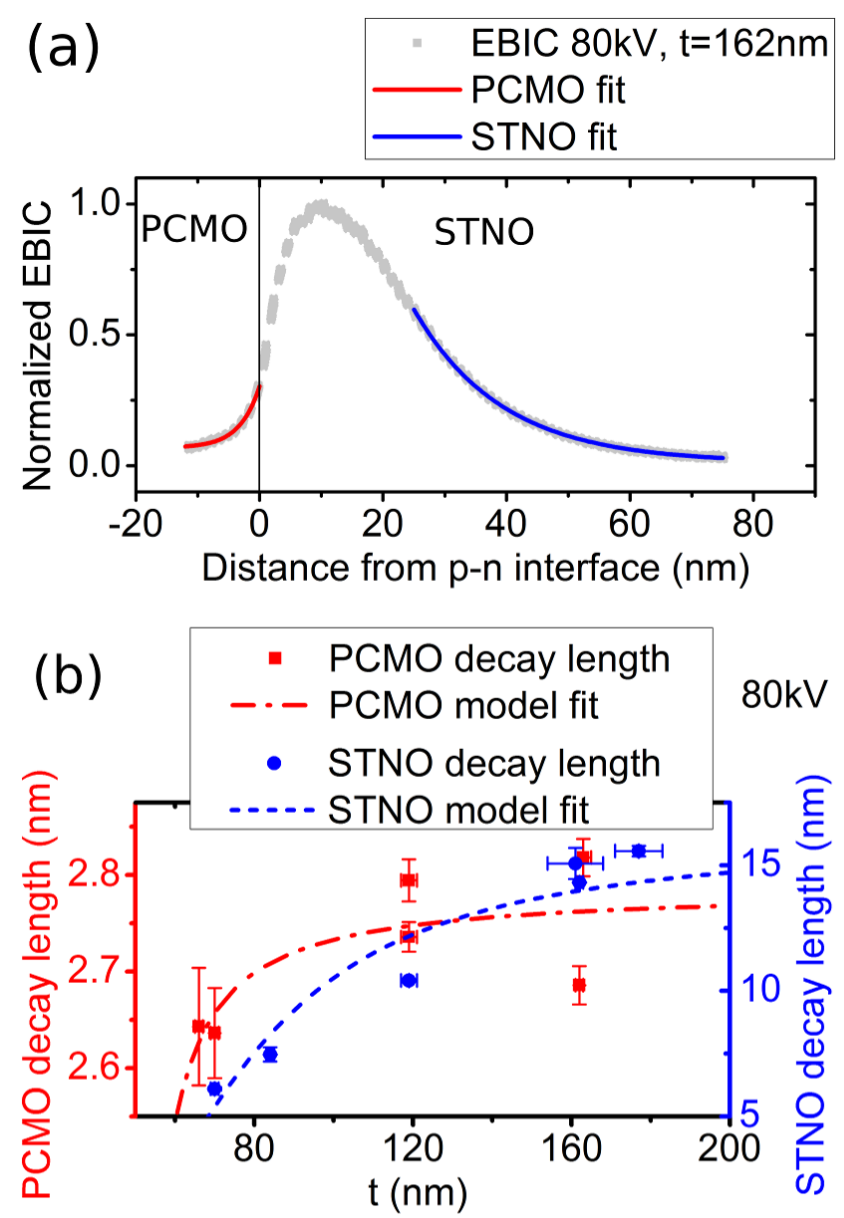

Figure 6.3: Decay lengths extracted from exponential fits of $80 \mathrm{kV}$ EBIC profiles. (a) Example of fits in the PCMO and STNO region; (b) decay lengths extracted from the fits showing the almost constant PCMO and increasing STNO values. The increase with increasing thickness can be attributed to the effect of recombination at the lamella surfaces. A simple model of the surface influence is fitted to the extracted decay lengths, from which we extract a $3 \mathrm{~nm}$ PCMO and a $17 \mathrm{~nm}$ STNO diffusion length, as well as $25 \mathrm{~nm}$ thick dead layers at the lamella surfaces.

Fig. 6.3 shows the results of exponential fits to the STEBIC profile, done independently for both sides of the p-n junction. The profiles' estimated inflection points were used as a starting point for the exponential fits, as they can roughly be attributed to the borders of the space charge region [141]. The fit results are roughly independent of thickness for PCMO, except for small thickness where they steeply decrease. For STNO the fitted decay lengths continuously increase with thickness above the minimal sample thickness required for measurable STEBIC signals. The highest combined decay length is remarkably similar to the combined diffusion length of $21 \mathrm{~nm}$ for PCMO and STNO, which was found in our earlier work using a simple collection efficiency model [12]. The thickness dependence suggests that the extracted decay lengths do not directly 
correspond to the respective diffusion lengths, which would be independent of thickness. As seen in Fig. 6.1 for $15 \mathrm{kV}$, in general an increasing sample thickness leads to an increasing generation volume lateral width, which could lead to broadening STEBIC profiles and thus increasing decay length. However, as explained from Fig. 6.2, we can exclude a thickness-dependent generation volume for $80 \mathrm{kV}$. Instead, we will argue and quantitatively analyze that the effect of surface recombination on the decay length is the underlying phenomenon. The impact of surface recombination on STEBIC profiles of a cross-section p- $n$ junction of finite thickness is discussed by Tan et al. [50] who provide an approximate analytic model with a large number of terms for the spatially dependent charge collection efficiency, which follows the shape of cross-section EBIC profiles in the point source case. They show an increasing decay length of the charge collection efficiency with increasing sample thickness, especially for thickness smaller than the bulk diffusion length. Furthermore, the effect is more pronounced for a large surface recombination velocity, strengthening the impact of the surface. This impact can be better understood by building on a simpler model for the effective diffusion length $L_{e f f}(z)$, accounting for the influence of charge carrier recombination at the beam entry surface, which was developed by Hackett [48] and Jastrzebski et al. [49] and further supported by Donolato 142 as well as Nichterwitz and Unold 47]. The effective diffusion length is equal to the extracted decay length $\lambda[142]$. The model is dependent on the depth $z$ in the sample and is valid for charge neutral surfaces. We use the same approach as Hackett and Jastrzebski et al., but propose an equation symmetric in $z$ to account for the beam exit surface as well. Furthermore, we account for the existance on 'dead' layers on both surfaces. These layers, resulting from FIB lamella preparation, are highly damaged sample regions where charge carriers immediately recombine and thus do not contribute to the EBIC signal. We found their thickness to be $t_{d} \leq 40 \mathrm{~nm}$ on each surface for PCMO-STNO in our earlier work [23], however the thickness is known to depend on the exact preparation method [130]. To include them in our model for $\mathrm{L}_{\text {eff }}$, we correct any term with the sample thickness $t$ in an expression with the dead layer thickness $t_{d} . t_{d}$ can thus be used as an additional fit parameter. Integrating the depth z over the whole sample thickness $t$, our approach leads to

$$
L_{e f f}(t)=L \sqrt{1-\frac{L}{\left(\frac{t}{2}-t_{d}\right) \cdot \frac{D}{s \cdot L}+\operatorname{coth}\left(\frac{\frac{t}{2}-t_{d}}{L}\right)}}
$$

with $L$ being the bulk diffusion length, $s$ the surface recombination velocity and $D$ the diffusion coefficient. This equation can be fitted to the thickness dependent decay lengths of PCMO and STNO separately. $L$ and $t_{d}$ were obtained from the fits for each material. The diffusion coefficients were fixed using the room temperature mobilities $\mu_{\mathrm{PCMO}}=0.02 \frac{\mathrm{cm}^{2}}{\mathrm{Vs}_{\mathrm{s}}}$ and $\mu_{\mathrm{STNO}}=1 \frac{\mathrm{cm}^{2}}{\mathrm{~V}_{\mathrm{s}}}$ with the Einstein relation $D=\frac{k_{B} T}{e} \mu[12$. Using a series of fits for different orders of magnitude of $\mathrm{s}$, the best results were obtained for $\mathrm{SPCMO}_{\mathrm{PCM}}=10^{3} \frac{\mathrm{cm}}{\mathrm{s}}$ and sSTNO $\geq 10^{6} \frac{\mathrm{cm}}{\mathrm{s}}$. The latter should be treated as lower limit. These values are in a reasonable range $-10^{6} \frac{\mathrm{cm}}{\mathrm{s}}$ is a common value for FIB-processed surfaces [31] - and indicate PCMO and STNO react differently to the focused ion beam preparation, leading to different recombination behaviour at their surfaces. The fit results obtained for these values of s are: $L_{\mathrm{PCMO}}=2.79(3) \mathrm{nm}, L_{\mathrm{STNO}}=16.7(9) \mathrm{nm}, t_{d, \mathrm{PCMO}}=24(7) \mathrm{nm}$ and $t_{d, \mathrm{STNO}}=25(3) \mathrm{nm}$. The dead layer thickness is roughly equal for both materials and is in accordance with the upper limit value of $40 \mathrm{~nm}$ from our earlier work [23. 
Both extracted diffusion lengths are smaller than the expected bulk values. Note that the STNO literature bulk value $(160 \mathrm{~nm})$ is questionable due to the actual mobility being strongly dependent on the doping procedure and the excess charge carrier lifetime being only estimated for our used doping [136]. Still, if the literature value is considered to be within the correct order of magnitude, it is comparable to the largest used lamella thickness, limiting the accuracy of the model described by Eq. 6.1 and leading to an underestimation of the determined diffusion length. However, this limitation is clearly not prevalent for PCMO due to the bulk diffusion length $(7 \mathrm{~nm})$ expected to be much smaller than the lamella thickness, and the literature mobility and lifetime values being more reliable $[12,13]$. It should be noted that the large 1 ns lifetime is only expected for the low temperature charge ordered PCMO phase, with a smaller value expected for room temperature 13. Thus, the smaller room temperature diffusion length found here for PCMO is reasonable. Still, a $3 \mathrm{~nm}$ diffusion length exceeds the extension of the PCMO space charge region, which is below $1 \mathrm{~nm}[9]$. This shows that the previously made conclusion from macroscopic current-voltage characteristics under optical excitation, stating that at room temperature only excess charge carriers generated at the PCMO-STNO interface contribute to the short circuit current, has to be modified to include generation from at least some nanometers into the PCMO bulk [14].

\subsubsection{EBIC maxima}

Fig. 6.4 (a) shows the thickness development of the STEBIC profile maxima for $80 \mathrm{kV}$ STEM. The maxima have been normalized by the used beam current. Note that measurements from two beam currents are included, 230 and $900 \mathrm{pA}$, which do not show significant differences in the normalized plot below $160 \mathrm{~nm}$ thickness, strongly indicating that the excitation is happening in the low injection regime 143. Starting from the minimum sample thickness of $t \approx 60 \mathrm{~nm}$, the EBIC maximum increases and saturates at about $120 \mathrm{~nm}$. This points to an influence of the sample surface, becoming more important for small sample thickness, where a large fraction of the generated electron-hole pairs are close to the surfaces and are thus more likely to recombine at surface defects. Furthermore, the onset of EBIC at $t \approx 60 \mathrm{~nm}$ is roughly equal to the double dead layer thickness $2 t_{d} \approx 50 \mathrm{~nm}$ as found from the decay length fits, indicating that no EBIC signal emerges from lamella regions with thickness $t \leq 2 t_{d}$. In the thickness regime above $160 \mathrm{~nm}$, the STEBIC profile maxima from high beam current data are significantly smaller than from low beam current data, which may be explained by a transition to high injection conditions [47].

Fig. 6.4(b) addresses the shift of the STEBIC profile maximum away from the chemical PCMO-STNO interface. The shift highlighted in the inset was determined as the difference of the PCMO-STNO interface and EBIC maximum positions. It clearly increases from about $4 \mathrm{~nm}$ for a sample thickness of $t=70 \mathrm{~nm}$ to about $10 \mathrm{~nm}$ for $t=160 \mathrm{~nm}$. Along with the maximum shift, the whole STEBIC profiles become broader to the STNO side with increasing sample thickness. A shift of the EBIC maximum away from the chemical interface in a cross-section geometry on such a short length scale has never been clearly shown before, as previous literature shows this effect - along with overall broadening EBIC profiles with increasing shift - only on the scale of more than $100 \mathrm{~nm}$ 47, 52. Both appoint a shift of the EBIC maximum as a result of excitation in the high injection regime. Furthermore, Nichterwitz and Unold also show increasing shift of the EBIC maximum position with decreasing doping on the lighter doped junction 


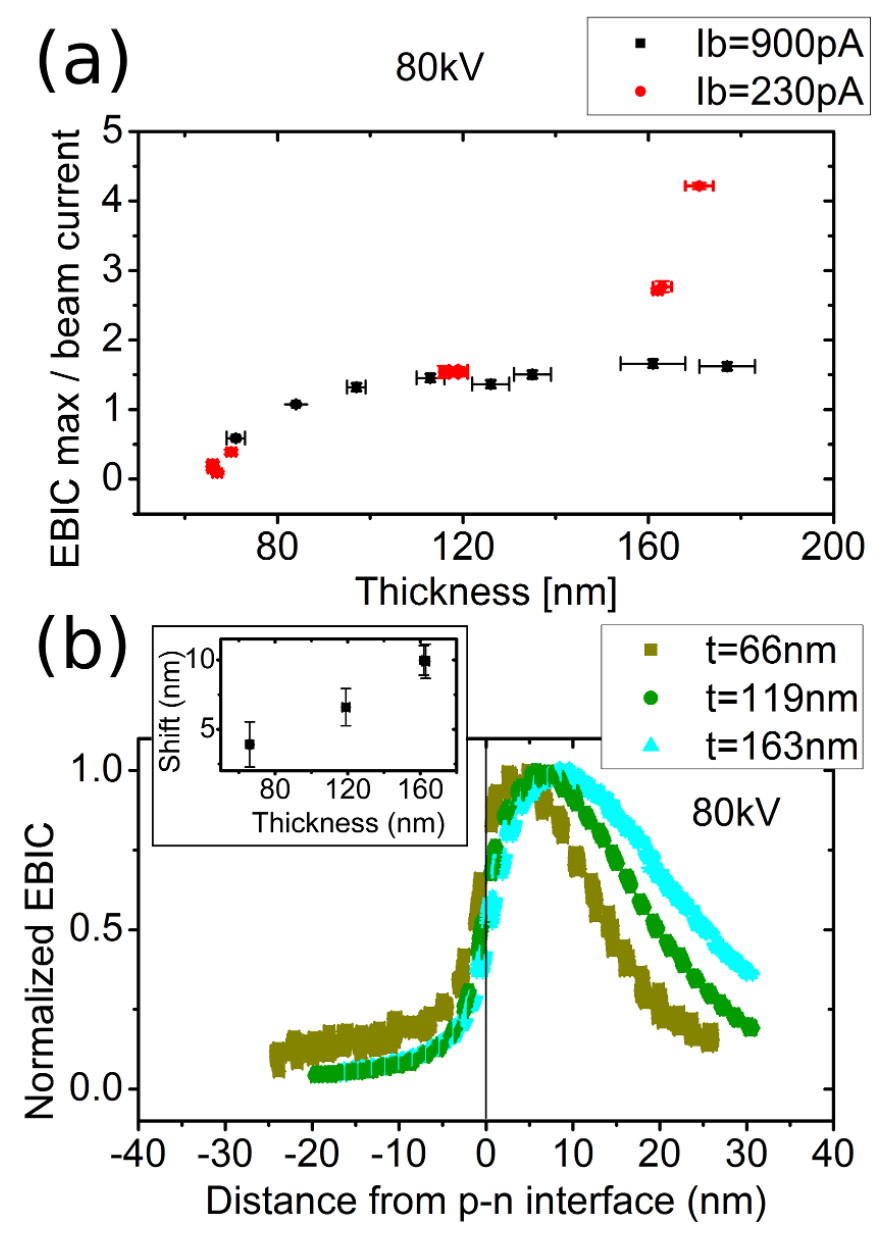

Figure 6.4: Characteristics of STEBIC profile maxima versus local lamella thickness for $80 \mathrm{kV}$ STEM: (a) Maximum values, normalized to beam current. The surface influence on the EBIC signal shows in the decrease of the signal maximum for small sample thickness. (b) Normalized STEBIC profiles show a shift of the maximum with increasing thickness, as highlighted in the inset, as well as overall broadening profiles.

side as well as with increasing diffusion length in their simulations [47]. As explained for Fig. 6.4(a), we can exclude a high injection effect at least in the thickness regime up to $120 \mathrm{~nm}$, thus we would like to elaborate on the doping influence and the diffusion length. We explain the small scale shift in our results by the asymmetric doping of our p- and n-doped materials. The p-doping in PCMO for $x=0.34$ is $N_{A}=6.3 \cdot 10^{21} \mathrm{~cm}^{-3}$, while the n-doping in STNO for $y=0.002$ is $N_{D}=3 \cdot 10^{19} \mathrm{~cm}^{-3}$. In this situation, a crucial parameter is the position of the intrinsic point, where $n_{0}=p_{0}$ in equilibrium, i.e. without electron beam excitation, where only a thermal generation rate $g_{t h} \propto p_{0} n_{0}$ of excess charge carriers is present. According to Smith, the smaller n-doping leads to a shift of the intrinsic point into the space charge region of the n-doped layer, compared to the case of equal doping where it would be at the chemical interface ( $\operatorname{shift}=0$ ) [33]. At the intrinsic point, the total number of equilibrium charge carriers $p_{0}+n_{0}$ reaches a minimum. Looking at the case of electron beam excitation, therefore, for a constant generation rate $g_{\text {exc }}$ of excess charge carriers (concentration $\delta$ ) across the space charge region, a local maximum injection is reached at the intrinsic point according to $g_{0}+g_{\text {exc }} \propto\left(p_{0}+\delta\right) \cdot\left(n_{0}+\delta\right)$. If we assume that recombination of excess charge carriers 
in the space charge region can be neglected, it follows directly that the STEBIC profile maximum must also be at this position. However, because the electric field is highest at the chemical interface, i.e. the assumption of recombination free transport of minority charge carriers across the interface is best fulfilled there, the STEBIC maximum might not be shifted as much as the neutral point. For our PCMO and STNO doping levels, we estimate from general heterojunction theory in box approximation [53, 144] that the intrinsic point is shifted not more than $10 \mathrm{~nm}$ into the STNO space charge region. The measurements approach this value for large thickness. Details of this behaviour are expected to depend on the dominating recombination mechanism in the used material, which is still to be determined for PCMO-STNO.

The thickness influence on the shift in the low injection regime can be qualitatively explained with the influence of the lamella surfaces on the thickness dependent effective diffusion length. An increasing effective diffusion length with thickness, which we find for STNO (Fig. 6.3), has the same effect as an increasing bulk diffusion length, leading to an increasing shift of the EBIC maximum position as shown in the simulations by Nichterwitz and Unold [47]. The STEBIC maximum position shift effect clearly needs a sophisticated model, which is, however, beyond the scope of this paper. We propose that such a model could provide a future prospect of investigating STEBIC signal around the intrinsic point, to map out the local injection of charge carriers in the space charge region, therefore accessing information about the band structure at the p-n junction interface. In the high injection regime, the shift may be increased due to changing shape of STEBIC profiles as nonlinear injection dependence on beam current comes into play 47.

\subsection{Summary}

To summarize, in photovoltaic systems with minority charge carrier diffusion lengths in the nanometer regime, it is not straightforward to investigate the p-n junction using usual SEM-based EBIC methods. On the example of a PCMO-STNO heterojunction, we compared cross-sectional STEBIC investigations by low energy SEM and high energy STEM methods. STEM provides a much better resolution as the electron beam generation volume is drastically reduced compared to SEM. Taking advantage of simultaneous EBIC and EELS mapping, it is possible to clearly find the position of the EBIC maximum being shifted away from the chemical interface to the lower doped sample side, showing the important role of the ratio of p- and n-doping concentrations. Using the enhanced resolution, we determined the charge carrier diffusion length to be $3 \mathrm{~nm}$ for PCMO and $17 \mathrm{~nm}$ for STNO. Surface effects can be approximately modeled using separate simple expressions for the space charge and the neutral region.

\subsection{Methods}

An epitaxial thin PCMO film (Ca doping $x=0.34$ ) was grown on STNO (Nb doping $y=0.002$ ) by ion beam sputtering, with $\mathrm{Au}$ and $\mathrm{Ti}$ contact layers. In the sample for $300 \mathrm{kV}$ measurements, Pt replaces Au as a contact material and an additional annealing step was performed for $20 \mathrm{~h}$ with an oxygen partial pressure of $10^{-4}$ mbar. More details on the preparation procedure can be found in our previous work [12. The SEM sample and the STEM sample for $300 \mathrm{kV}$ were prepared with a $100 \mathrm{~nm}$ thick PCMO film, 
whereas the STEM sample for $80 \mathrm{kV}$ was prepared with a $370 \mathrm{~nm}$ PCMO film. The SEM lamella was prepared inside the SEM sample by Focused Ion Beam (FIB) in an FEI Nova Nanolab 600 Dual Beam FIB microscope, resulting in a wedge shaped lamella, as described in detail in our previous work [23. The STEM lamellas were taken out of the other samples by FIB (see e.g. [127] for a detailed description), including a part of the $\mathrm{Au} / \mathrm{Pt}$ front contacts. The $80 \mathrm{kV}$ lamella was put upside down on a dummy sample, on which a $\mathrm{Ti}$ and a protective Au layer were sputtered afterwards, resulting in a well-defined back contact layer to the STNO on the lamella. Subsequently, the lamella was transferred to a DENSsolutions MEMS chip and fixed at two of the chip's Pt contacts with FIB deposited Pt-C patches. In contrast, the lamella from the $300 \mathrm{kV}$ sample, including the front contact, was directly transferred to a DENS chip, and larger Pt-C stripes were deposited to simultaneously fix the lamella on the chip contacts and provide a back contact to the STNO. On the chips trenches were cut by FIB into the lamellas to provide a fixed current path. Finally, the lamellas were thinned on the chips by FIB. The $80 \mathrm{kV}$ lamella was thinned in a wedge shape with a range of thickness at the PCMOSTNO interface from about 60 to $180 \mathrm{~nm}$, while the $300 \mathrm{kV}$ lamella was prepared with a homogeneous interface thickness of about $80 \mathrm{~nm}$ (see below for details of the thickness measurement). For thinning, a $30 \mathrm{kV}$ ion beam was used, with a final cleaning step at $5 \mathrm{kV}$. All experiments were conducted at room temperature.
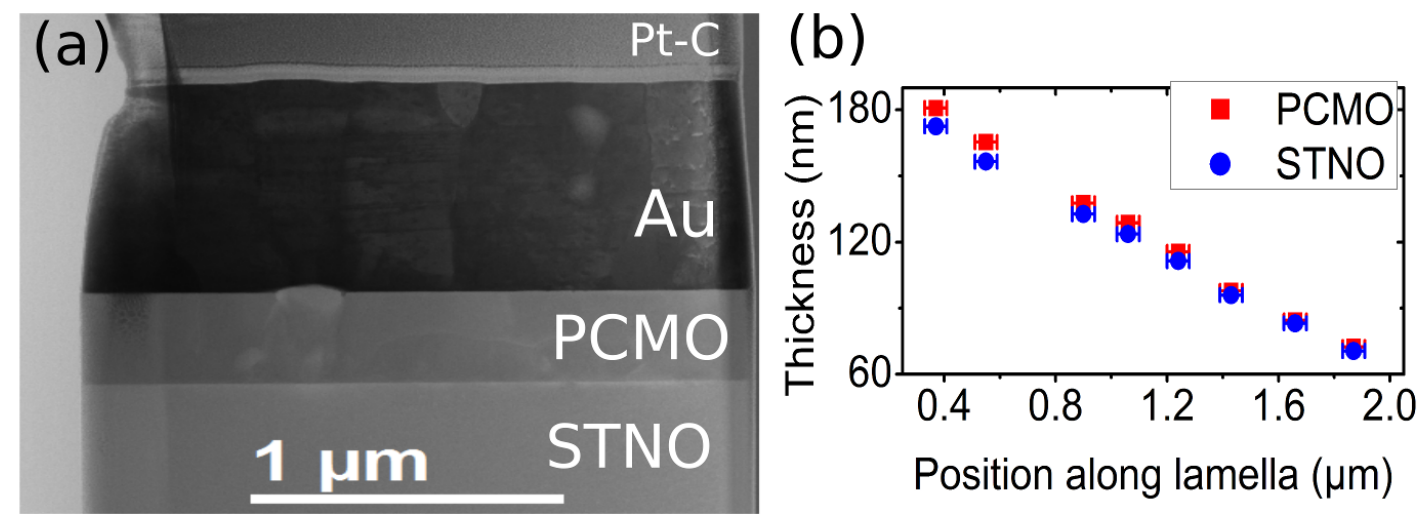

Figure 6.5: (a) Low magnification bright field STEM image of the used cross section lamella for $80 \mathrm{kV}$ STEM measurements, (b) corresponding average thickness profile of PCMO and STNO, showing the wedge shape of the lamella. While the relative errors of the thickness measurement method are small, an additional systematic error of $20 \%$ of the measured thickness (not shown) affects quantitative comparison with other methods.

Fig. 6.5(a) shows an overview of the used wedge shaped STEM lamella. The SEM lamella and the $300 \mathrm{kV}$ STEM lamella with constant thickness of about $80 \mathrm{~nm}$ are not shown. The SEM sample was investigated at $15 \mathrm{kV}$ acceleration voltage in an FEI NanoSEM 650 using standard Gatan EBIC equipment, including a Stanford SR570 preamplifier directly connected to the FEI image recording software, providing synchronous beam movement and EBIC signal collection. An FEI Titan 80-300 TEM was used in STEM mode with a DENSsolutions D7+ Lightning contacted sample holder. For the $80 \mathrm{kV}$ measurement, the current measurement was done by a Keithley setup including a type 6517A electrometer, connected to STEM measurements via a custom National Instruments LabVIEW recording software. This software needed manually synchronization of 
the STEM bright field signal to the EBIC measurement, which was done by comparing the measurement timestamps in both setups. For the $300 \mathrm{kV}$ measurement, an advanced setup was used, connecting the SR570 to the Lightning holder and using a Keithley 2000 digital multimeter for amplifier voltage output readout, which was controlled by another custom LabVIEW program. A custom Gatan DigitalMicrograph script enabled direct synchronization of the electron beam position to the multimeter readout. In STEM, we focused on obtaining highly magnified local EBIC profiles, averaged over 1-6 lines. Along with bright field STEM and EBIC signal, low loss EELS spectra were recorded by a built-in Gatan GIF Quantum 965ER in the energy range from 0 to $185 \mathrm{eV}$ with dispersion $0.1 \mathrm{eV}$ per channel, as exemplified in Fig. 6.6(a). For $80 \mathrm{kV}$, the STEM beam diameter was estimated to be $1 \mathrm{~nm}$ from a bright field STEM image recorded under the same conditions as the STEBIC data, estimating the transition width of the sample to vacuum at the surface of a thin FIB-deposited Pt-C layer region. For $300 \mathrm{kV}$, atomic resolution bright field STEM could be achieved, indicating a beam diameter of smaller than $0.2 \mathrm{~nm}$. Measurements were drift-corrected in situ by a standard cross-correlation procedure supplied by Gatan.

The SEM lamella thickness profile was determined from a top view secondary electron image, as described in detail in our previous work [23]. The STEM lamella thickness profile was determined from the EELS low-loss spectra. Compared to the SEM methods, this method is directly connected to the STEM and EBIC measurements and thus more direct. We evaluated the local STEM lamella thickness using the log-ratio method providing the local sample thickness in units of the electron inelastic mean free path [96]. The latter was computed for our materials using the Malis method 145] for our experimental setup and $80 \mathrm{kV}$ to be $M F P_{\mathrm{PCMO}}=61(12) \mathrm{nm}$ and $M F P_{\mathrm{STNO}}=64(13) \mathrm{nm}$, averaged over a range of roughly $100 \mathrm{~nm}$. The averaging of the local sample thickness for each profile results in a small statistical error of some nanometers. While these relative thickness errors are small, the systematic errors of the method are significantly higher, which should be kept in mind when comparing the thickness measurements from different methods quantitatively, e.g. from SEM images. The Malis method provides the electron inelastic mean free path with a systematic error of $20 \%$, propagating to the absolute sample thickness. Consequently, the thickness of different positions along one lamella can be clearly separated, while the thickness comparison between SEM and STEM lamellas is affected by the systematic errors. The resulting STEM lamella thickness profile, including just the relative thickness errors, is shown in Fig. 6.5(b).

The position of the chemical interface between PCMO and STNO was estimated from the SEM secondary electron signal line profiles by calculating the point of $50 \%$ of the contrast change across the interface. For the STEM-based STEBIC measurement, the interface position was extracted from Mn-M edge signal profiles acquired from the synchronized low loss EELS measurements, as shown in Fig. 6.6. The Mn-M edge onset is located at an energy loss of $49 \mathrm{eV}$. A power law background in the range $38-45 \mathrm{eV}$ was subtracted and the remaining signal from 45 to $115 \mathrm{eV}$ was integrated for each beam position. From the resulting EELS Mn-M edge profiles, the same method as for SEM was used to obtain the interface position.

The characteristic STEBIC profile shape is shown in Fig. 6.2. The position and signal height of the EBIC maximum was obtained by averaging all values at the profile signal peak and their distance from the chemical interface position, respectively. To extract the decay lengths $\lambda_{\mathrm{PCMO}}$ and $\lambda_{\mathrm{STNO}}$, the exponential function fits to the STEBIC profiles 


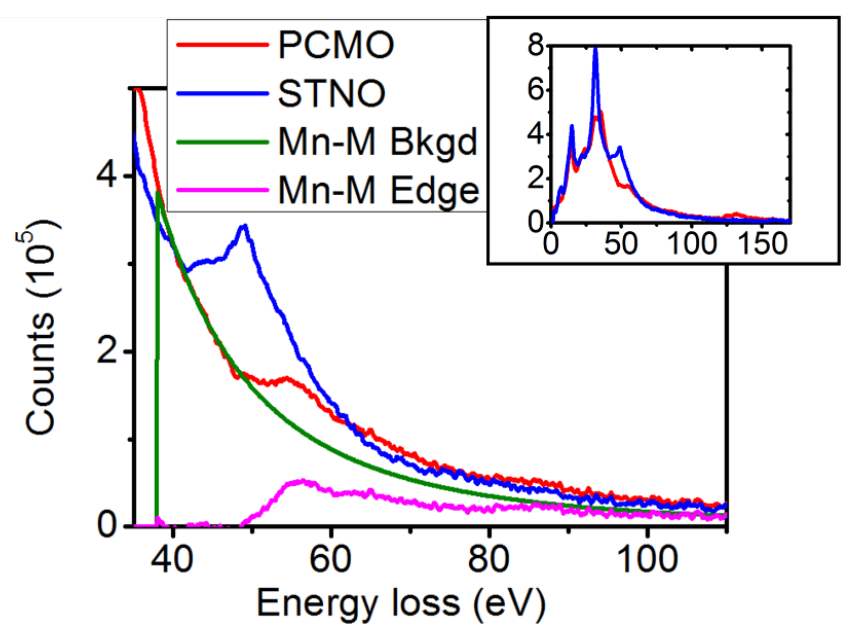

Figure 6.6: Low loss EELS data recorded along with STEM-based STEBIC: Mn-M edge signal, which is only present in PCMO, with subtracted power law background. The inset shows typical spectra from PCMO and STNO on a larger energy loss scale.

were done independently for PCMO and STNO, starting on each junction side's estimated inflection point and using the function $f(x)=a \cdot \exp \left( \pm \frac{x-x_{0}}{\lambda}\right)+y_{0}$, with the sign varied depending on the junction side. $x_{0}, y_{0}$, a and $\lambda$ were used as fit parameters. $x_{0}$ accounts for the starting point of the exponential drop and $y_{0}$ for background signal far away from the space charge region.

\subsection{Acknowledgement}

The authors thank Emanuel Franke for additional contact preparation and Vladimir Roddatis for help with and valuable discussion about TEM experiments. Funding of the Deutsche Forschungsgemeinschaft through SFB1073 (projects B02 and Z02) is acknowledged.

\subsection{Contributions}

PCMO-STNO p-n heterojunctions were prepared by Benedikt Ifland and Christian Jooss. Additional contact preparation was performed by Emanuel Franke. LabVIEW control software was written by Jonas Lindner. FIB preparation and experiments were performed by the author and Tobias Meyer with help from Jonas Lindner and Vladimir Roddatis. Simulations and analysis were performed by the author under guidance of Michael Seibt. Results were discussed by the author, Tobias Meyer, Christian Jooss, Michael Seibt and Vladimir Roddatis. Figures were made and text was written primarily by the author, with text contributions by Michael Seibt. 


\section{Chapter 7}

\section{EBIC temperature behaviour, space charge region and modeling of a PCMO-STNO p-n heterojunction}

Patrick Peretzki, Tore Niermann, Corin Jung, Benedikt Ifland, Christian Jooss and
Michael Seibt

\subsection{Premise}

Building on the work from [12], [14] and Ch. 6, we extend the parameter space of PCMO-STNO STEBIC experiments, show a way to directly measure the space charge region size and provide STEBIC modeling. An analysis of cross section scanning transmission electron beam induced current (STEBIC) results from a set of electron beam acceleration voltages and beam currents is given, as well as investigation of behaviour at low temperatures, showing the impact of the charge ordered PCMO phase. Electron holography is used to estimate the space charge region size from the internal electronic potential with near-nanometer resolution. Two methods are shown as stepping stones to model experimental STEBIC profiles; one using a simple model of a collection function convoluted with a simulated electron beam generation volume, the other using finite element simulations to model collection behaviour of the sample.

\subsection{Methods}

The PCMO-STNO samples were prepared as described in [12]. The FIB preparation of cross section lamellas was done with an FEI Nova NanoLab 600 dual beam microscope with a $30 \mathrm{kV}$ ion beam, and a fine cleaning step performed at $5 \mathrm{kV}$. Details of the preparation method can e.g. found in [127]. Lamellas were dug out, but left in the sample to simplify contacting. The lamella for room temperature measurements was thinned down to a constant thickness of about 200-400 nm. During the STEBIC measurement series with a beam current and acceleration voltage variation, the beam entrance surface was cleaned again with a $5 \mathrm{kV}$ ion beam showering after each complete scan of the lamella in order to clean a thin layer of carbon-containing absorbants, which were deposited during the scan because of cracked gas molecules in the remaining gas environment. As quite high electron beam currents were used, this effect was pronounced and could be monitored by decreasing layer contrast in secondary electron images and decreasing maxima in 
STEBIC images. After the cleaning step, the initial SE contrasts and STEBIC maxima were restored. This was possible in one measurement session as STEBIC measurements were carried out in the FIB in SEM mode. However, the lamella thickness might be slightly reduced during these processes, approaching the given $200 \mathrm{~nm}$ lower limit. The lamella for cooling experiments was prepared using similar ion beam parameters, but in a wedge shape with a maximum thickness of $\mathrm{t} \approx 710 \mathrm{~nm}$. Fig. 7.1(a) shows the lamella in cross section view. The thickness profile was measured from contrast jumps in a top view image of the lamella, as described in [23. The determined profile is shown inf Fig. 7.1(b).

The lamella for holography was also prepared similarly, but after the final $30 \mathrm{kV}$ thinning step, the lamella was additionally thinned in the center region directly from the top in order to produce a trench next to a clean area. Afterwards, another $30 \mathrm{kV}$ cleaning step was performed to provide optimal conditions for holography, which requires a thin residual layer on the lamella beam entrance and beam exit surfaces in order to short-circuit the p-n junction so that no charging and only the electrostatic potential influences the holograms. The interface between the sample system and the trench in the lamella is shown in Fig. 7.1(c).

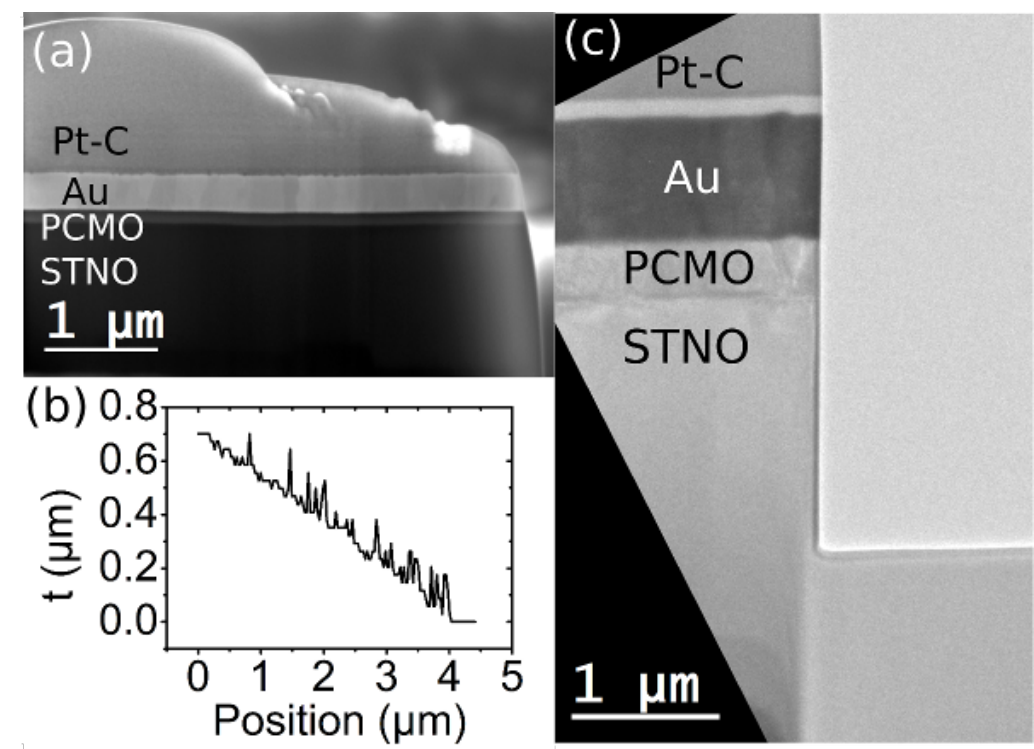

Figure 7.1: Lamellas for STEBIC and holography prepared by FIB. (a) STEBIC lamella in cross section SEM view. It was prepared in a wedge shape, with the thickness gradient shown in (b). (c) shows a TEM image of the holography lamella, where a trench was prepared by FIB during the thinning process, providing a clear reference point for empty holograms near the sample system to ensure good conditions for amplitude and phase image reconstruction.

The STEBIC measurements in SEM were carried out in the described FIB as well as in a FEI NanoSEM 650 by standard Gatan EBIC equipment including a Stanford Research Systems SR570 current preamplifier. Current-voltage characteristics were recorded with the same setup using a voltage source included in the current preamplifier. Electron beam scans were done in a high magnification, with the cross section lamella surface tilted by 85 degrees, i.e. almost perpendicular, to the electron beam, and the beam rotated in order to scan perpendicular to the PCMO-STNO interface. To ensure high 
spatial resolution, images were collected in immersion mode. Note that the absolute value of the EBIC maximum might considerably underestimate the real values, as the current preamplifier is influenced by a finite input impedance and limited bandwidth in the used high amplification settings. Still, the shown currents are correct relative to each other, as the sample amplification range was used for all of them.

The in-situ cooling was performed in the NanoSEM using an EBIC-compatible Gatan model C1002 cooling stage with liquid nitrogen cooling. In this setup, an electric heater with variable current is used by a temperature control system to heat against the liquid nitrogen cooling in order to establish a stable temperature. With this setup, a stable temperature within a range of $1 \mathrm{~K}$ can be established for several hours.

Off-axis holography [105] was performed using the FEI Titan 80-300 Berlin Holography Special TEM, which includes a spherical aberration corrector providing a spatial resolution of $75 \mathrm{pm}$ at $300 \mathrm{kV}$ acceleration voltage. Holography is performed using a double Möllenstedt biprism setup with a holography transfer lens. The TEM was operated in Lorentz mode, i.e. imaging with the Lorentz lens instead of the switched off objective lens, in order to increase the hologram field of view to the $\mu \mathrm{m}$ range [146]. The holograms were recorded with a lamella tilt several degrees away from zone axis conditions in order to avoid dynamic diffraction effects. Dose rates of $60-85 \frac{\text { electrons }}{\mathrm{nm}^{2} \mathrm{~s}}$ were used, and several holograms averaged over several images with in-between drift correction and an effective exposure time of $4-7 \mathrm{~min}$.

The electron beam generation volume in single layer samples was simulated by the Monte Carlo based program CASINO 3.3 developed by Demers et al. [108]. We used the parameters $1 \mathrm{~nm}$ beam diameter and 200000 simulated impinging electrons. The spatial energy distribution was calculated with $2 \mathrm{~nm}$ lateral and $10 \mathrm{~nm}$ depth spatial resolution. The energy loss calculation was calculated using the Joy-Luo model [110]. The resulting three-dimensional energy distribution is averaged laterally in order to provide radial and depth information. For the collection efficiency model, multilayer samples were simulated using CASINO 2.42 [107]. For these simulations, a larger beam diameter $(20 \mathrm{~nm})$ was used.

Finite element simulations were carried out with COMSOL 5.2a, in order to determine the spatial distribution the electronic potential $V$ and electron and hole concentrations $p$ in the simulated sample. A model for a $\mathrm{p}-\mathrm{n}$ homojunction with different doping levels and different electron and hole mobilities was developed. In the first step, the PoissonBoltzmann equation

$$
-\frac{d^{2} V}{d x^{2}}=\frac{e}{\varepsilon} \cdot\left(n_{i} \cdot \exp \left(-\frac{e \cdot V}{k_{B} \cdot T}\right)-n_{i} \cdot \exp \left(\frac{e \cdot V}{k_{B} \cdot T}\right)+C_{i o n}\right)
$$

with

$$
C_{\text {ion }}= \begin{cases}-N_{A}, & \text { p-doped region } \\ N_{D}, & \text { n-doped region. }\end{cases}
$$

was solved to determine $V$ in a one-dimensional sample with a maximum element size of $0.1 \mathrm{~nm}$ in the mesh. In a second step, the stationary Poisson equation and charge carrier 
equations for electrons and holes

$$
\begin{aligned}
-\nabla^{2} V & =\frac{e}{\varepsilon} \cdot\left(p-n+C_{i o n}\right) \\
\vec{\nabla} \cdot\left(-D_{p} \vec{\nabla} p-\mu_{p} p \vec{\nabla} V\right) & =\frac{G}{E_{G}}-B \cdot\left(n p-n_{i}^{2}\right) \\
\vec{\nabla} \cdot\left(-D_{n} \vec{\nabla} n+\mu_{n} n \vec{\nabla} V\right) & =\frac{G}{E_{G}}-B \cdot\left(n p-n_{i}^{2}\right)
\end{aligned}
$$

were solved simultaneously for a two-dimensional sample (see Fig. 7.2), with a minimum and maximum element size of $7.5 \mathrm{pm}$ and $2 \mathrm{~nm}$, respectively, using an adaptive mesh refinement routine. The results from the first step were used as initial values in the second step. The same length $l_{x}=l_{p}+l_{n}$ as in the one-dimensional sample was used, as well as a given thickness $l_{y}=t$. The second step also incorporates an electron beam generation volume $G$, which is realized as an approximate "stripe source" of electrons and holes, i.e. a Gaussian shape (full with at half maximum $2 \mathrm{~nm}$ ) in $x$-direction and a constant over the whole sample thickness in $y$-direction, with a given generation rate $G_{p n}$.

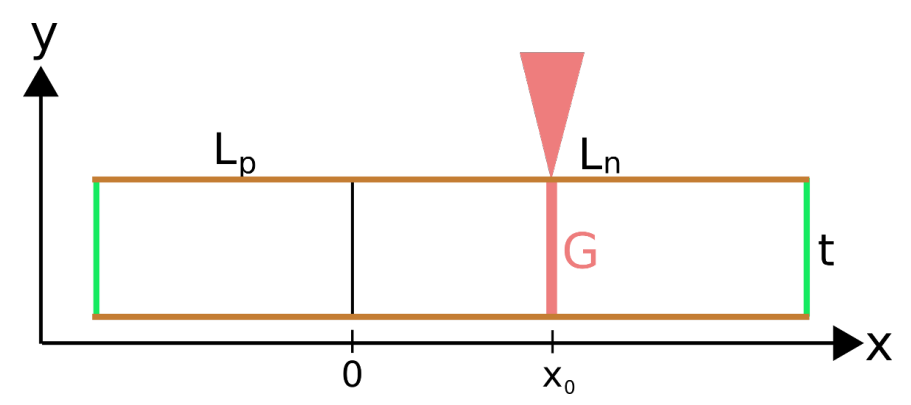

Figure 7.2: Two-dimensional geometry for the COMSOL simulation. The sample length in $x$ direction is split into the length of the p-doped region $l_{p}$ and the $\mathrm{n}$-doped region $l_{n}$. The length in y-direction is equal to the sample thickness $t$. The electron beam, leading to a stripe-shaped generation volume $G$, is scanned along the sample for several positions $x_{0}$ to produce STEBIC profiles. The contacts are marked on the left and right end and the (beam entry and exit) surfaces on the top and bottom end of the sample.

As boundary conditions, the potential was set to the values $V_{0}$ and $V_{D}$ at the left and right contact, respectively (Dirichlet boundary conditions), and recombination equations

$$
\begin{aligned}
\vec{\eta} \cdot\left(-D_{p} \vec{\nabla} p-\mu_{p} p \vec{\nabla} V\right) & =S \cdot \delta p \\
\vec{\eta} \cdot\left(-D_{n} \vec{\nabla} n+\mu_{n} n \vec{\nabla} V\right) & =S \cdot \delta n
\end{aligned}
$$

were used for excess electron and hole concentrations (Neumann boundary conditions) at the contacts (with contact recombination velocity $S=S_{\text {cont }}$ ) and the surfaces (with surface recombination velocity $S=S_{\text {surf }}$ ). These conditions are only defined in a direction $\vec{\eta}$ normal to the contacts or surfaces. The excess concentrations are defined from the results from models 1 and 2 by $\delta p=p_{2}-p_{1}$ and $\delta n=n_{2}-n_{1}$. Similarly to these equations, the electron beam induced current was extracted from the simulation results at one of the contacts via

$$
E B I C=\int_{0}^{t} e \cdot S_{\text {cont }} \cdot(\delta p-\delta n) \mathrm{d} y .
$$


The used parameters in the model are shown in Table 7.1. $k_{B}$ is the Boltzmann constant, $e$ the elemental charge and $\varepsilon_{0}$ the vacuum permittivity. The intrinsic charge carrier concentration was calculated as $n_{i}=2\left(\frac{2 \pi m_{e} k_{B} T}{h}\right)^{1.5} \exp \left(\frac{-E_{G}}{2 k_{B} T}\right)$ for the homojunction, $m_{e}$ being the free electron mass, and $h$ the Planck constant. The acceptor and donor doping concentrations $N_{A}$ and $N_{D}$ as well as the recombination coefficient $B$ were varied, as explained in Sec. 7.3 .

\begin{tabular}{l|c|c} 
Parameter & Symbol & Value \\
\hline Temperature & $T$ & $300 \mathrm{~K}$ \\
Length p-doped & $l_{p}$ & $250 \mathrm{~nm}$ \\
Length n-doped & $l_{n}$ & $800 \mathrm{~nm}$ \\
Thickness & $t$ & $100 \mathrm{~nm}$ \\
Permittivity & $\varepsilon$ & $11.7 \varepsilon_{0}$ \\
Hole mobility & $\mu_{p}$ & $200 \mathrm{~cm}^{2} /(\mathrm{V} \cdot \mathrm{s})$ \\
Electron mobility & $\mu_{n}$ & $1100 \mathrm{~cm}^{2} /(\mathrm{V} \cdot \mathrm{s})$ \\
Hole diffusion constant & $D_{p}$ & $k_{B} T \mu_{p} / e$ \\
Electron diffusion constant & $D_{e}$ & $k_{B} T \mu_{n} / e$ \\
Generation rate & $G_{p n}$ & $10^{30} \mathrm{eV} /(\mathrm{cm} \cdot \mathrm{s})$ \\
Bandgap & $E_{G}$ & $1.1 \mathrm{eV}$ \\
Contact recombination velocity & $S_{c o n t}$ & $10^{8} \mathrm{~cm} / \mathrm{s}$ \\
Surface recombination velocity & $S_{s u r f}$ & $10^{5} \mathrm{~cm} / \mathrm{s}$ \\
Intrinsic charge carrier concentration & $n_{i}$ & $1.43 \cdot 10^{9} \mathrm{~cm}-3$ \\
Bulk majority hole concentration & $p_{p, 0}$ & $N_{A}$ \\
Bulk majority electron concentration & $n_{n, 0}$ & $N_{D}$ \\
Potential offset & $V_{0}$ & $-\frac{k_{B} T}{e} \ln \left(p_{p, 0} / n_{i}\right)$ \\
Diffusion potential & $V_{D}$ & $\frac{k_{B} T}{e} \ln \left(p_{p, 0} n_{n, 0} / n_{i}^{2}\right)$
\end{tabular}

Table 7.1: Parameters used in COMSOL model

\subsection{Experimental results}

Fig. 7.3 shows a current-voltage characteristics (I-V curve) of the sample used for STEBIC experiments in the SEM, recorded by the EBIC setup, but without electron beam illumination, i.e. in the dark. As expected, the data generally follow the Shockley theory [34. The slope of the curve in forward direction (positive voltage) is the inverse serial resistance $R_{S}$ of the sample, whereas the slope in reverse direction (negative voltage) is the inverse sum of serial and parallel resistance $R_{p}$ [147]. Fitting linear functions in both direction yields the values $R_{p}=3.313(3) \mathrm{k} \Omega$ and $R_{s}=13.79(2) \Omega$. The former is in the range determined for similar samples at room temperature in a dedicated electrical characterization setup [12], while the latter is about two orders of magnitude larger than the value measured in the dedicated setup, but also seems reasonable given the probable connection weaknesses from cables, silver paint contacts and the sample holder, as well as the macroscopic contact having been slightly damaged during contacting. 


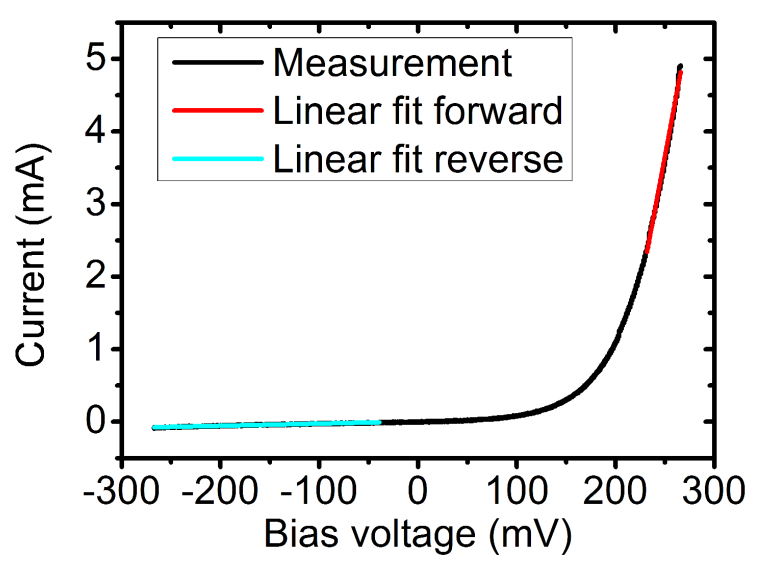

Figure 7.3: Current-voltage characteristics in the dark of the sample used for STEBIC measurements. Linear fits in forward and reverse direction enable determination of the parallel resistance $R_{p}=3.313(3) \mathrm{k} \Omega$ and the serial resistance $R_{\mathrm{s}}=13.79(2) \Omega$

\subsubsection{Room temperature STEBIC experiments}

The beam current dependence of the maximum EBIC signal near the PCMO-STNO interface in the 200-400 nm thick STEBIC sample is shown in Fig. 7.4(a). A linear increase with incrasing beam current is exhibited for all acceleration voltages, which strongly indicates the experiment is conducted in the low injection regime [143]. As similar or even lower beam currents, along with similar lamella thickness were used in the temperaturedependent measurements as well, we can conclude the same for these experiments. Note that the apparent slope of the linear increase varies with acceleration voltage. It increases from 2 to $10 \mathrm{kV}$ and decreases again from 10 to $30 \mathrm{kV}$. This demonstrates the influence of the limited lamella thickness: For acceleration voltages higher than $10 \mathrm{kV}$, a large part of the electron beam generation volume is outside the sample and does not contribute to excitation of electron hole pairs, which reduces the overall EBIC signal. The effect increases with even further increasing acceleration voltage, leading to the further decreasing slope for acceleration voltages above $10 \mathrm{kV}$. The same behaviour can be found in the Monte Carlo simulations of the cumulative energy dose deposited in the sample, see Fig. 7.4(b), supporting this interpretation. The simulation was done for PCMO, however the difference to STNO is marginal.

Fig. 7.5 (a) shows the STEBIC profile shape dependence on acceleration voltage. The profile width is indicated by the decay length on the PCMO as well as the STNO side. As the profiles show, the decay length on both sides increases with the acceleration voltage up to $10 \mathrm{kV}$, but decreases for higher voltages. As for the change in maximum behaviour described before, the finite lamella thickness in the STEBIC experiment is the primary reason, as a large part of the generation volume is outside the lamella for high acceleration voltages. This is shown by the Monte Carlo simulation of the generation volume width, see. Fig. 7.5(b). The width of the lateral energy distribution is calculated using the interquartile range (IQR) for each depth $z$. For the depth range corresponding to the experimentally used sample thickness, as shown by the marked region, the qualitative width behaviour is the same as the decay length behaviour in the STEBIC profiles.

Furthermore, up to $5 \mathrm{kV}$, the width barely exceeds $100 \mathrm{~nm}$, which is the PCMO layer 

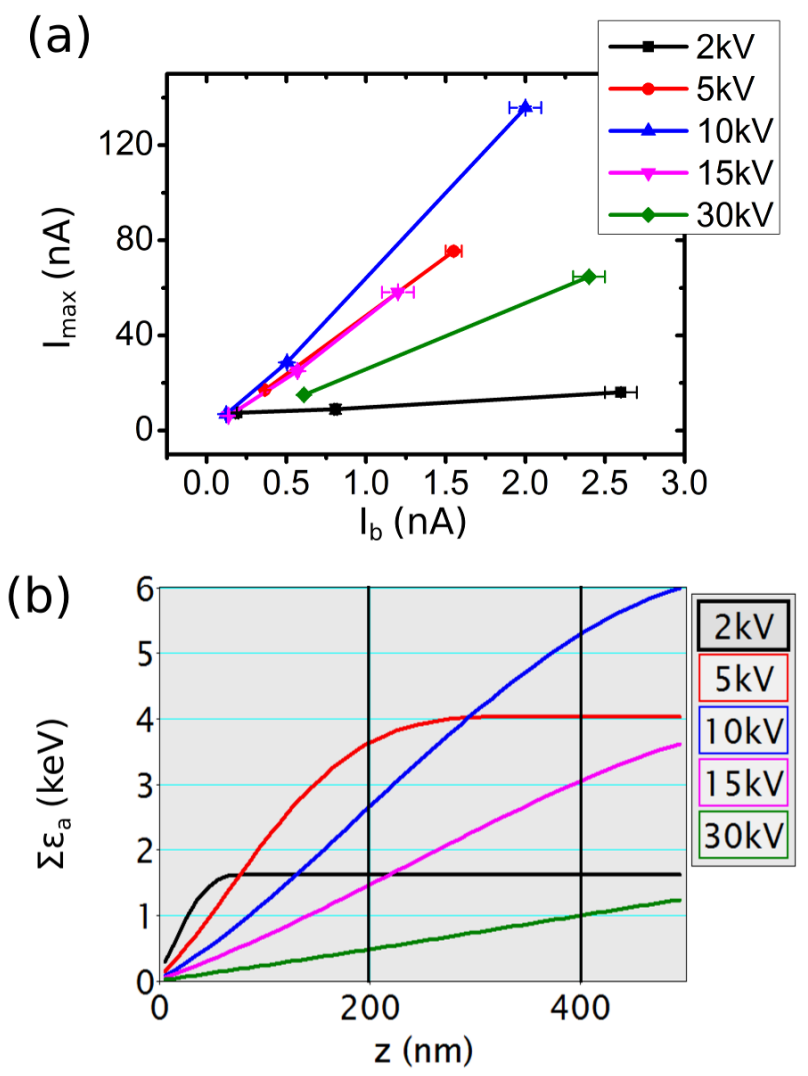

Figure 7.4: (a) Beam current influence on the maximum EBIC signal in STEBIC measurements, for a constant lamella thickness of 200-400 nm. A linear behaviour throughout the used beam current and acceleration voltage range strongly indicates the experiments were conducted in the low injection regime, and the changing slope with changing acceleration voltage points to the influence of the finite sample thickness in STEBIC measurements. (b) CASINO simulations of the deposited energy depth dose in PCMO for an incoming electron beam with the same acceleration voltage range. The ordinate indicates the deposited energy accumulated up to the given depth z. Comparing the accumulated energy in the depth corresponding to the experimental sample thickness (marked region in (b)) to the EBIC maximum slope from (a), a similar qualitative behaviour can be found for experiment and simulation.

thickness. This explains why for smaller acceleration voltages, no significant charge carrier numbers can be collected by the PCMO-STNO interface when exciting in the $\mathrm{Au}$ layer, as shown by the corresponding STEBIC profiles. Diffusion through the whole $\mathrm{PCMO}$ layer is not possible as the diffusion length is limited to a few nanometers (see Ch. 6). For higher acceleration voltages ( $10 \mathrm{kV}$ or more), the generation volume is broad enough to allow excitation at the charge collecting interface even when the electron beam hits the lamella in the Au layer, therefore the Au-PCMO interface is pronounced in these profiles. 

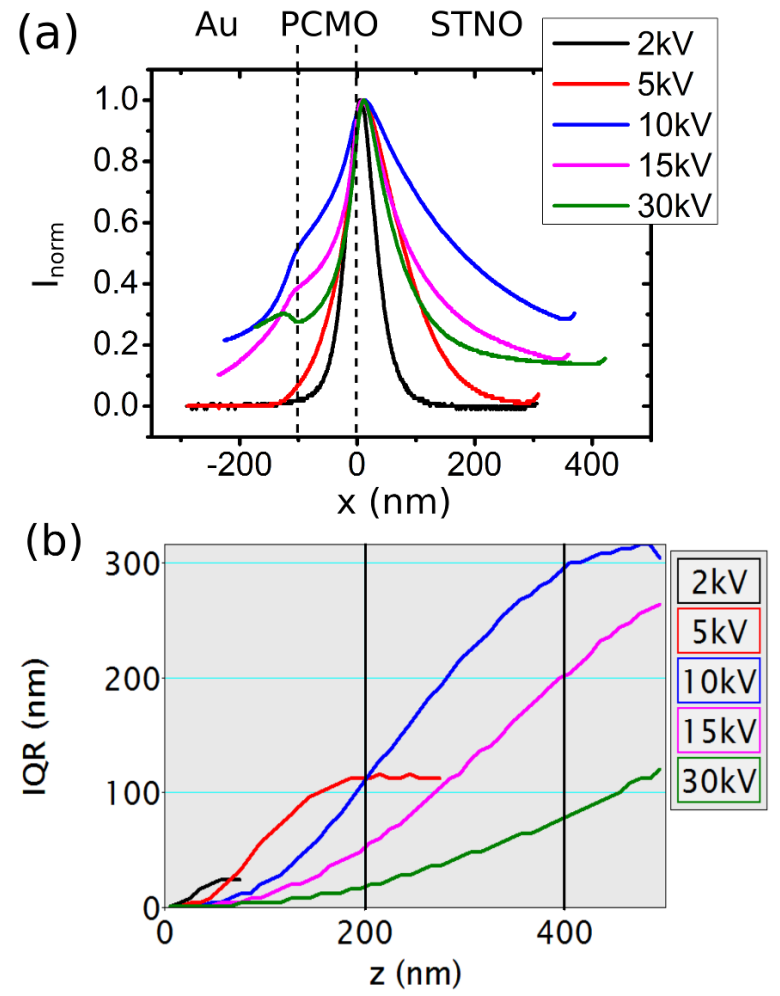

Figure 7.5: (a) Acceleration voltage dependence of STEBIC profiles, normalized to their maximum current. The profiles' decay length on both the PCMO and the STNO side changes the used acceleration voltage. The finite sample thickness shows up in the decay length decrease above $10 \mathrm{kV}$. (b) CASINO simulations of the electron beam generation volume width in $\mathrm{PCMO}$, calculated here as interquartile range (IQR) of the lateral energy distribution for $z=$ const., for the experimentally used acceleration voltage range. The width for the experimental sample thickness (marked range) shows the same qualitative behaviour as the decay length of the STEBIC profiles. Note that (a) the Au-PCMO interface is pronounced as a kink in the profiles only for acceleration voltages where (b) the generation volume is broad enough to excite electron hole pairs at the charge collecting PCMO-STNO interface even when the electron beam hits the lamella at the Au-PCMO interface.

\subsubsection{Low temperature STEBIC experiments}

Another lamella, processed into a wedge shape with thickness up to $710 \mathrm{~nm}$, was in-situ cooled during STEBIC experiments. STEBIC maps were collected with $5 \mathrm{kV}$ and $15 \mathrm{kV}$ acceleration voltage using three different electron beam currents (temperature dependent data was only collected with the smallest beam current in order to exclude any high injection regime effects). Cooling was done until liquid nitrogen temperature $\mathrm{T} \approx 80 \mathrm{kV}$, however stable SEM mapping could only be achieved down to T $\approx 160 \mathrm{~K}$. The sample was cooled down from room temperature rapidly, then slowly heated up again while collecting STEBIC maps. Before and after reaching room temperature, two comparative STEBIC maps were taken to check for a possible persistent effect of the cooling.

The behaviour of the STEBIC profile maxima with local lamella thickness is shown in Fig. 7.6(a). The analysis is similar to our previous work, which showed qualitatively similar results to those presented here for $5 \mathrm{kV}$ as well as $15 \mathrm{kV}$ [23]. The shape of the 

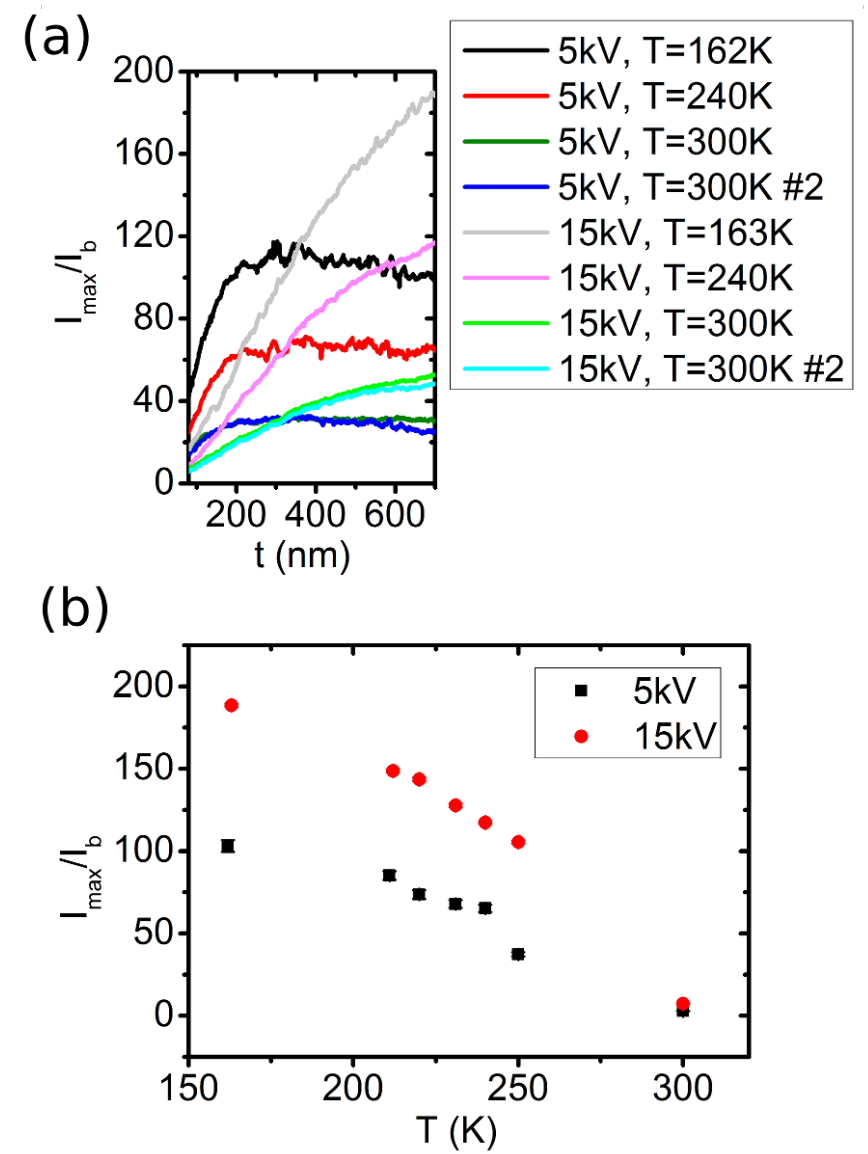

Figure 7.6: EBIC profile maximum behaviour with temperature and local lamella thickness, normalized by beam current, for 5 and $15 \mathrm{kV}$ acceleration voltage. (a) The thickness behaviour is qualitatively different for the acceleration voltages, but similar for different temperatures. The second room temperature result was recorded after cooling, showing no significant effect. (b) The temperature behaviour is qualitatively similar for both acceleration voltages, showing the transition into the increased photovoltaic effect in the low temperature charge ordered phase.

thickness behaviour is dominated by the depth dose of the generation volume. Here we add temperature as an additional parameter, showing generally increasing STEBIC maxima $I_{\max }$ with decreasing temperature. However, the qualitative thickness behaviour of the maxima is not influenced. Again, this points to the dominating influence of the generation volume. The two measurements at room temperature before and after the cooling cycle show no persistant cooling effects. In Fig. 7.6(b), the average maximum STEBIC signal for each temperature at high thickness is shown. As the $15 \mathrm{kV}$ maxima start to saturate at a higher thickness than the $5 \mathrm{kV}$ maxima, the average was calculated for the thickness range $\mathrm{t}=500-710 \mathrm{~nm}$ for $5 \mathrm{kV}$ and for only $\mathrm{t}=700-710 \mathrm{~nm}$ for $15 \mathrm{kV}$. Results confirm the increasing STEBIC maxima with decreasing temperature. The qualitative behaviour is similar for $5 \mathrm{kV}$ and $15 \mathrm{kV}$.

We explain the rise of the STEBIC signal at lower temperatures with the transition to the charge ordered phase at $\mathrm{T}_{\mathrm{CO}} \approx 240 \mathrm{~K}$, which is expected to show a larger PCMO minority charge carrier lifetime than at room temperature, and thus also a larger diffusion length, leading to higher EBIC signal [13]. However, the macroscopic short circuit cur- 
rent of PCMO-STNO p-n junctions measured for polychromatic light illumination shows a decreasing signal with decreasing temperature [12, 14]. The reason for the contradicting trends of EBIC and optical experiments remains to be explained. Furthermore, the increase in STEBIC maxima starts for higher temperatures than $\mathrm{T}_{\mathrm{CO}}$, which could be due to the rather broad temperature range of the phase transition [86, 148 150]. As the experiments in [12, 14] were conducted during cooling from room temperature, whereas ours were conducted during heating up from $100 \mathrm{~K}$, the higher apparent transition temperature found here could also be due to hysteretic effects of the first order phase transition. Additionally, it could hint at a possibly inaccurate temperature measurement, as the temperature in our setup is measured at the sample holder rather than the lamella.
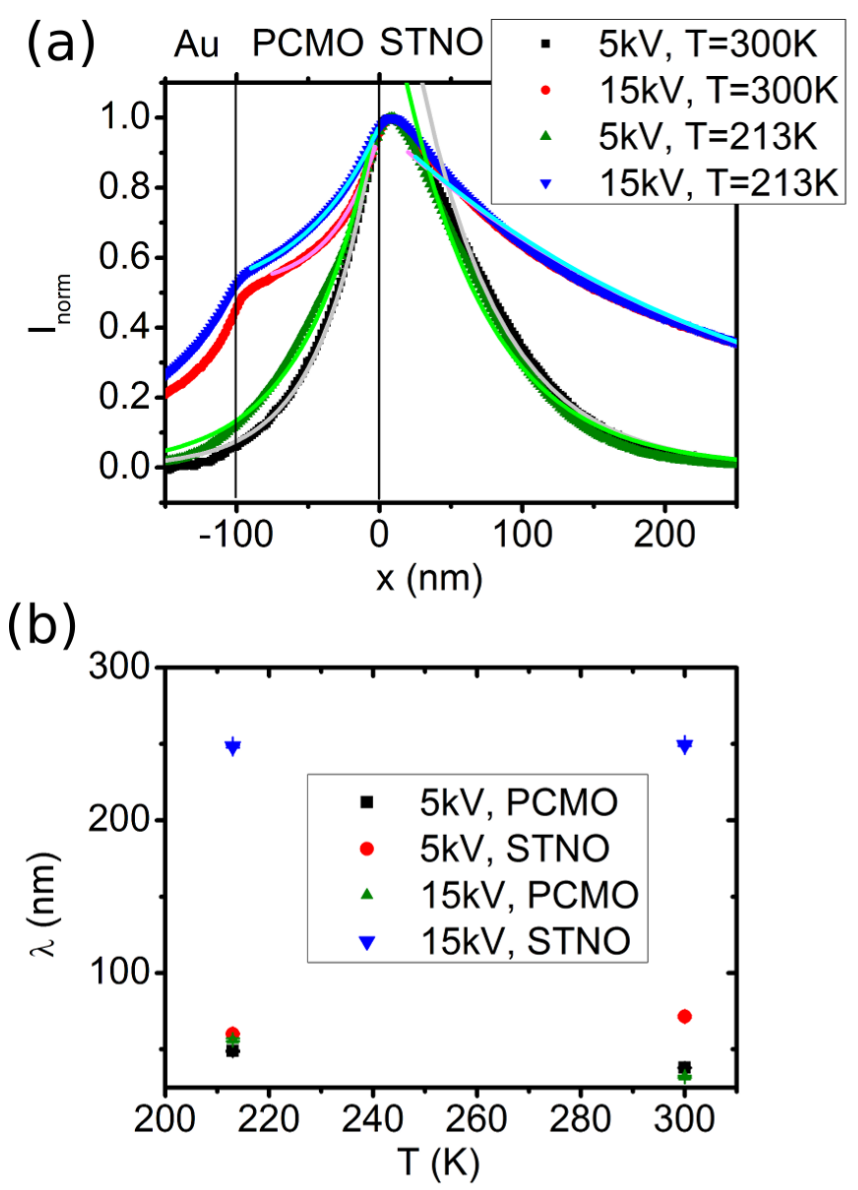

Figure 7.7: Fitting exponential decays to EBIC profiles at different temperatures. (a) Although the fit shows small errors, the fits do not reproduce the actual profiles very well, (b) Extracted decay length hints at a smaller PCMO decay length at room temperature than at $213 \mathrm{~K}$.

As a means of getting direct access to the diffusion length, generally a good way is to fit the STEBIC profiles with exponential functions, separately on the PCMO and the STNO side, as was also done in our previous work (see Ch. 6). However, the extracted decay constant $\lambda$ cannot readily be interpreted as a diffusion length, as the influence of the lamella surfaces and the generation volume has to be included [22]. The decay length may instead be described as an effective diffusion length, especially dependent 
on sample thickness. Still, both influences are essentially independent of temperature. Therefore, in order to confirm qualitatively an increasing PCMO diffusion length in the low temperature charge ordered phase, we propose the effective diffusion length as a first means. The results of a fit for room temperature as well as for the lowest evaluated temperature $\mathrm{T} \approx 160 \mathrm{~K}$ is shown in Fig. 7.7. Fig. 7.7(a) shows the resulting fit functions with the STEBIC profiles, normalized to their maximum. Note that the functions do not reproduce the behaviour of the experimental data very well, especially for STNO, despite having small fitting errors as shown in Fig. 7.7(b) (the errors are indiscernably small on the used scale). Because of the bad fits, we do not detail on fitting the STEBIC profiles of other temperatures.

Still, note that in Fig. 7.7(a), in the whole PCMO area the low temperature signal is higher than the room temperature signal, while in the STNO area they are identical for both temperatures. This may be connected to the extracted decay lengths in Fig. 7.7(b), showing that, while the STNO fit results do not significantly change between the two temperatures, the PCMO results are smaller at room temperature for both acceleration voltages.

As an alternative way of estimating the temperature influence on the STEBIC profiles, we propose a 'decay integral' $\Sigma_{\lambda}$. For each junction side, we integrate the STEBIC signal within a given range $\mathrm{L}_{\text {lim }}$ from the STEBIC profile maximum $\mathrm{x}_{\max }$ to the end of the range $\mathrm{x}_{\max } \pm \mathrm{L}_{\mathrm{lim}}$ and normalize it by the maximum STEBIC signal. This can be described by

$$
\begin{aligned}
\Sigma_{\lambda, \mathrm{PCMO}} & =\sum_{x=x_{\max }-L_{l i m}}^{x_{\max }} \frac{I(x)}{I_{\max }}, \\
\Sigma_{\lambda, \mathrm{STNO}} & =\sum_{x=x_{\max }}^{x_{\max }+L_{l i m}} \frac{I(x)}{I_{\max }} .
\end{aligned}
$$

The concept of the decay integral, albeit phenomenological, is similar to a decay length, but independent of the actual data shape. Furthermore, it is statistically more robust, which is why we favor it in this situation.

Fig. 7.8(a) and (b) show the thickness dependent decay integral for PCMO and STNO, with temperature and acceleration voltage as additional parameters. The $15 \mathrm{kV}$ decay integrals show a stronger thickness dependence than the $5 \mathrm{kV}$ decay integrals, which is explainable by the stronger influence of the generation volume for $15 \mathrm{kV}$. In fact, the shape is quite similar to that of the STEBIC maxima in Fig. 7.6(a)). For $5 \mathrm{kV}$ as well as $15 \mathrm{kV}$, and for all thicknesses, the PCMO decay integral at room temperature is smaller than the PCMO decay integrals of all lower temperatures. Note that decay integral thickness profiles for STNO are almost constant for all temperatures. This behaviour is emphasized more clearly in Fig. 7.6(c), where the decay integrals for large thickness have been averaged, similar to Fig. 7.6(b).

This behaviour confirms a larger PCMO diffusion length at temperatures below room temperature, although the diffusion length cannot be determined quantitatively from this procedure, and is in agreement with the results from the decay length fits from Fig. 7.7. Contrary to the STEBIC maximum temperature behaviour in Fig. 7.6, there is no gradual increase with decreasing temperature. Instead, the decay integral data hint at a more abrupt change in diffusion length at some temperature between $250 \mathrm{~K}$ and $300 \mathrm{~K}$, which could be explained by a hysteretic effect as described before.

Fig. 7.9 (a) shows beam current dependent decay integrals at room temperature. For 

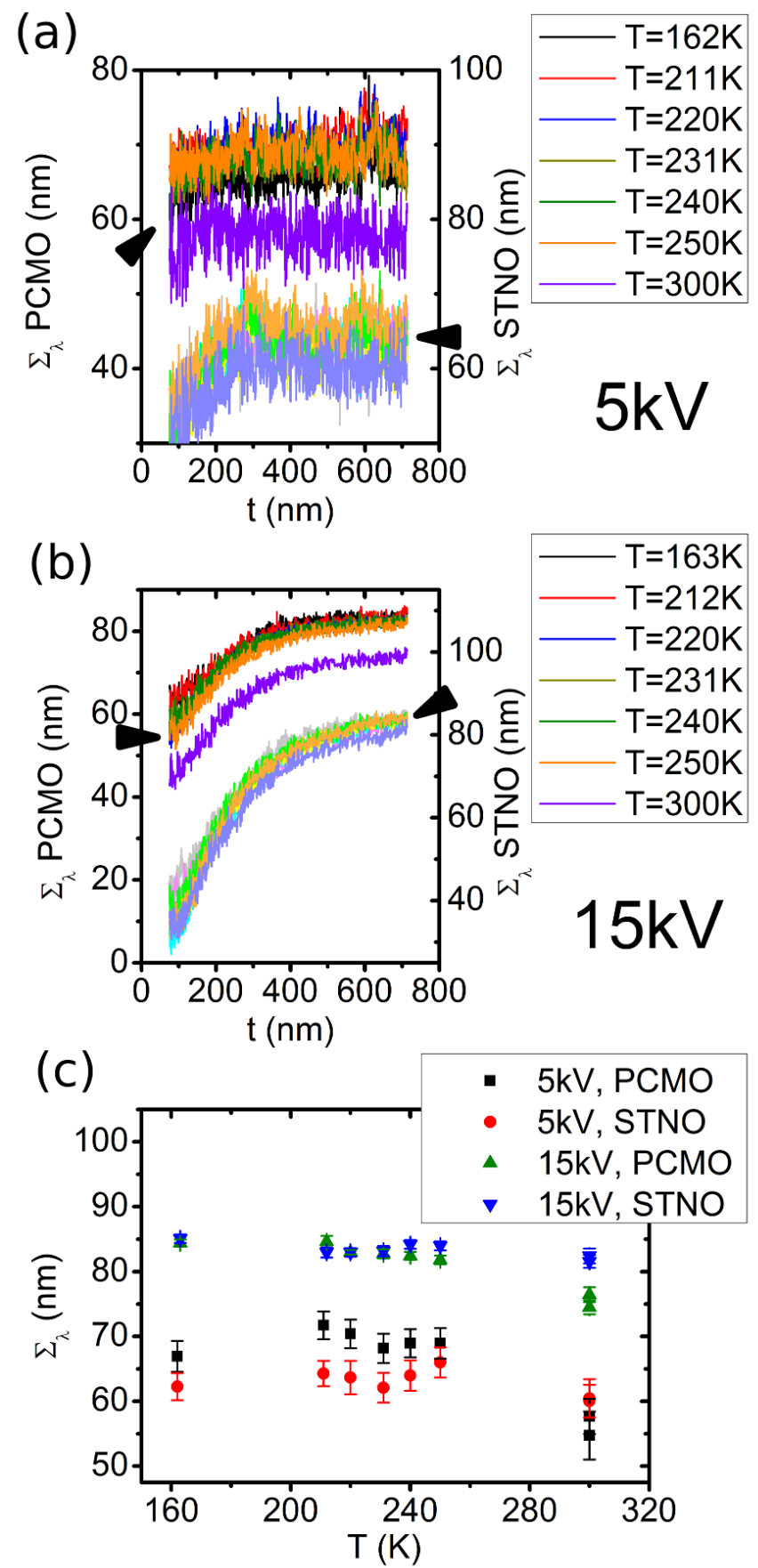

Figure 7.8: The decay integral provides an alternative method to estimate the decay shape of EBIC profiles, shown dependent on the local lamella thickness and temperature. (a) $5 \mathrm{kV}$ and (b) $15 \mathrm{kV}$ results all show a decreasing decay integral with decreasing thickness, hinting at the generation volume influence. While there is no clear temperature difference for STNO, the PCMO room temperature decay integral is significantly smaller than the low temperature results, providing a strong hint of decreasing PCMO diffusion length at room temperature due to melting of the low temperature charge ordered phase. (c) shows averaged results for large thickness, i.e. $\mathrm{t}=500-710 \mathrm{~nm}$ for $5 \mathrm{kV}$ and $\mathrm{t}=700-710 \mathrm{~nm}$ for $15 \mathrm{kV}$. 
(a)
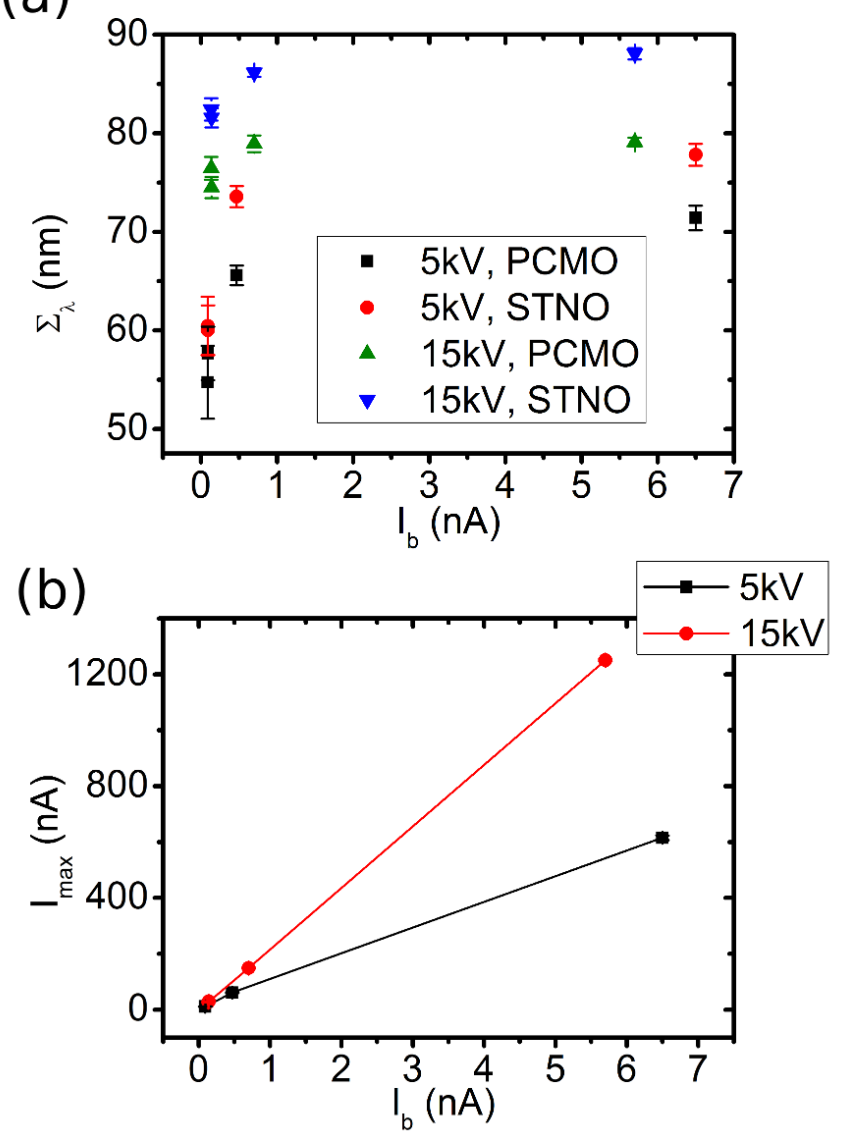

Figure 7.9: Beam current dependent EBIC at room temperature. (a) The decay integral of PCMO and STNO shows a significant drop for the lowest beam current $\mathrm{I}_{\mathrm{b}}=90 \mathrm{pA}$ and $140 \mathrm{pA}$ for $5 \mathrm{kV}$ and $15 \mathrm{kV}$, respectively. This might hint at a transition from low to high injection regime. (b) EBIC profile maximum dependence on beam current, averaged for large lamella thickness, i.e. $\mathrm{t}=500-710 \mathrm{~nm}$ for $5 \mathrm{kV}$ and $\mathrm{t}=700-710 \mathrm{~nm}$ for $15 \mathrm{kV}$. The $5 \mathrm{kV}$ EBIC maximum does not increase completely linear with the beam current, which could support the injection regime transition.

both acceleration voltages and junction sides, the decay integral is significantly lower at the lowest of the three used beam currents compared to the other beam currents. Still, the change is on the order of $5-10 \mathrm{~nm}$. This indicates a small change in the shape of STEBIC profiles, which could hint at a transition from low to high injection level regime between the first and the second beam current. In fact, looking at the mean STEBIC profile maxima for large sample thickness, as shown in Fig. 7.9(b), a slight change in slope between these currents can be found there as well, which is a typical sign for a change between the regimes in EBIC experiments [52]. Note that the smallest beam current values are estimated, as a high amplifying range of the current amplifier had to be used for them, decreasing the measured signal by a factor because of limited performance. To correct this effect, the data for the medium beam current were measured in both amplifying ranges, and low beam current data multiplied by the ratio of the two medium beam current measurements. Furthermore, the data set is very small, so that a transition between injection regions cannot clearly be determined. 


\subsubsection{TEM holography experiments}

TEM amplitude and phase images were reconstructed from averaged off-axis holograms. This was achieved by using the usual Fourier filtering based reconstruction method involving correction by empty reference holograms, i.e. from electrons not perturbed by the sample [146]. The chosen cut-off Fourier frequency determined a spatial resolution of about $10 \mathrm{~nm}$. A resulting pair of reconstructed amplitude and phase image is shown in Fig. 7.10(a) and (b). In both images, an extended defect is visible at the surface of the PCMO layer, which we identify to likely be a $\mathrm{MnO}_{z}$ precipitate, which have been described for non-annealed PCMO layers elsewhere [113]. This region was excluded from further analysis in order to only examine pure PCMO and STNO.

The phase $\phi$ of TEM images is, in theory, proportional to the electrically active local sample thickness tact and the local electrostatic potential $\mathrm{V}$, providing access through the latter from phase images. Using a fixed interaction constant $\sigma_{\mathrm{IA}}=0.0065 \frac{\mathrm{rad}}{\mathrm{V} \cdot \mathrm{n} m}$ for $300 \mathrm{kV}$, the simple connection of these quantities is

$$
\phi=\sigma_{\mathrm{IA}} \cdot V \cdot t_{\mathrm{act}},
$$

which is for example described by Park et al. [103]. Looking at phase profiles across the PCMO-STNO p-n junction, averaged along the p-n junction plane, we obtain results as shown in Fig. 7.10(c). Also shown are profiles from relative thickness maps obtained from EFTEM images of the same region in the same lamella, which can be used to describe the thickness influence on the phase image.

The qualitative trend in the region about $100 \mathrm{~nm}$ away from the p-n junction interface is similar for thickness and phase, confirming the thickness influence on the phase in this region. Furthermore, the qualitative trend of all averaged phase profiles is very similar. We expect a space charge region size below $1 \mathrm{~nm}$ for PCMO and of $27 \mathrm{~nm}$ for STNO from box approximation considerations [9]. Outside of this region, we expect flat potential. In our experimental results, the potential indeed follows the same trend as the thickness outside of this region. However, the exact interpretation of the signal within the space charge region is difficult. Simply using equation 7.10 to extract the potential leads to the result shown in Fig. 7.10(d). For this, the relative thickness measurement was converted to an absolute thickness by multiplying with the inelastic mean free path $\mathrm{MFP}_{\mathrm{PCMO}}=117(12) \mathrm{nm}$ and $\mathrm{MFP}_{\mathrm{STNO}}=123(13) \mathrm{nm}$ in the respective region, which were calculated for $300 \mathrm{kV}$ EFTEM measurements using the Malis method [145].

As expected, flat potential is reached far away from the p-n interface $(\mathrm{x}=0)$. Looking closer at the region near the space charge region, the qualitative trend is as expected from the potential in a space charge region, also including a potential jump at the interface due to the different mean inner potential of the regions. Still, on the STNO side flat potential is only reached at $x \geq 100 \mathrm{~nm}$, which is much more than the expected space charge region side. On the PCMO side, the situation is similar with a flat potential only reached for $x \leq-100 \mathrm{~nm}$, whereas we would expect that no band bending is visible at all due to the exceptionally small space charge region.

We expect drawbacks from the limited resolution of only $10 \mathrm{~nm}$ in the phase images and from the manually corrected material jump in the EFTEM images, which also have a limited resolution. Furthermore, in Fig. 7.10(c) the visible phase increase approaching the p-n interface from the PCMO side is rather small, and only enhanced from the seemingly decreasing thickness slope approaching the interface, putting the resulting potential rise in Fig. 7.10 (d) into doubt. On the other hand, the phase drop when 


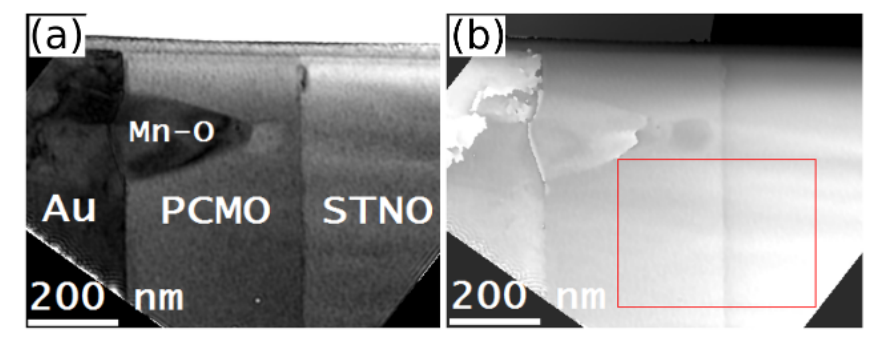

(c)

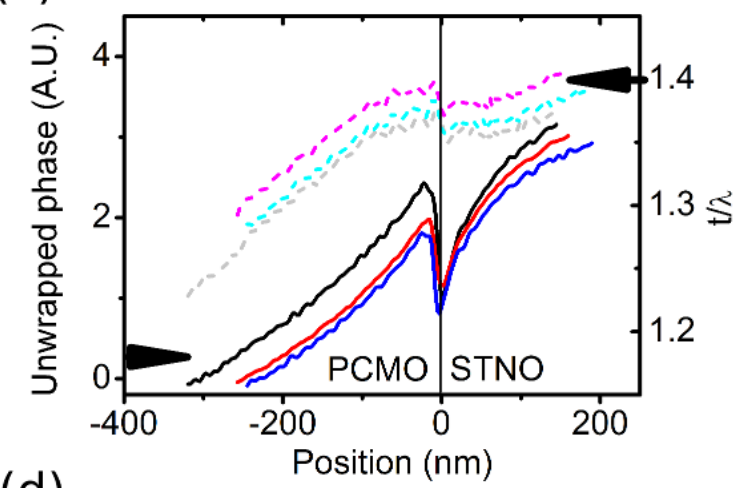

(d)

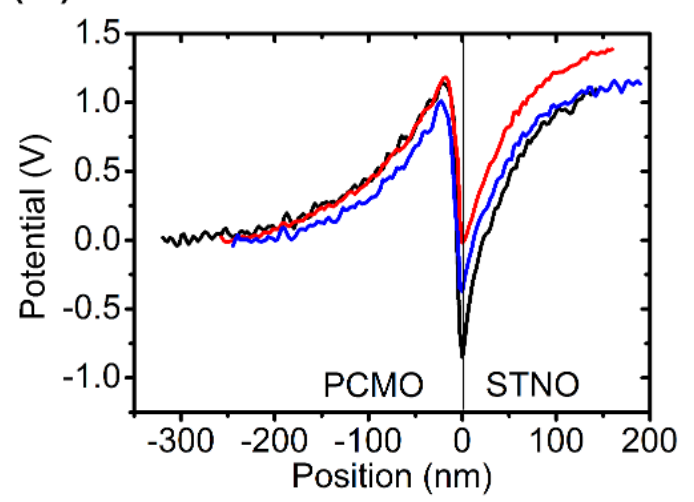

Figure 7.10: Results from electron hologram reconstructions. (a) Amplitude image of the used lamella, showing an included Mn-O precipitate at the PCMO layer surface, (b) corresponding unwrapped phase image, marking the region where profiles were averaged along the p-n junction plane, (c) phase and EFTEM thickness profiles from different measurements, in units of the inelastic mean free path $\lambda$, (c) potential profiles, which are the phase divided by thickness profiles and an interaction constant. The potential jump at the $\mathrm{p}-\mathrm{n}$ junction $(\mathrm{x}=0)$ is clearly visible. The potential rise on both junction sides is on a larger scale than expected from box approximation space charge region size.

approaching the interface from the STNO side is strongly pronounced, supporting that it is really connected to a change in electrostatic potential due to space charge. 


\subsection{Modeling}

\subsubsection{Collection efficiency model}

A simple model for describing the shape of cross section EBIC profiles is by weighting the space charge region and the neutral region of the sample differently. The model is described in 45] and was applied by us to PCMO-STNO STEBIC measurements before [12. In this model, we assume a collection efficiency profile perpendicular to the p-n junction interface. In the space charge region, we assume that the electric field is strong enough that all minority charge carriers reaching this region are collected by the junction, and thus set the collection efficiency to 1 . In the neutral region, diffusion is the dominant reason for minority charge carrier movement, and we assume an exponentially decreasing collection efficiency when increasing the distance to the space charge region, with the decay length being the diffusion length. Therefore we define the collection efficiency function $f_{\mathrm{c}}$ stepwise:

$$
f_{c}(x)= \begin{cases}\exp \left(\left(d_{\mathrm{PCMO}}+x\right) / L_{\mathrm{PCMO}}\right), & \mathrm{x} \leq-d_{\mathrm{PCMO}} \\ 1, & -d_{\mathrm{PCMO}}<x \leq d_{\mathrm{STNO}} \\ \exp \left(-\left(d_{\mathrm{STNO}}-x\right) / L_{\mathrm{STNO}}\right), & x>d_{\mathrm{STNO}}\end{cases}
$$

To simulate a STEBIC profile, the collection function may be convoluted with the Monte Carlo simulated generation volume, which is summed up in the directions parallel to the p-n junction interface, generating the generation function $g(x)$. The convolution process for the discrete simulated data may be described by

$$
I_{\text {sim }} \propto \sum_{x^{\prime}} g\left(x, x^{\prime}\right) f_{c}\left(x^{\prime}\right)
$$

In general, for each excitation position $x$, a separate generation volume has to be used to account for the position in the multilayer sample. The proportionality constant must include the excitation energy of electron hole pairs and the beam current.

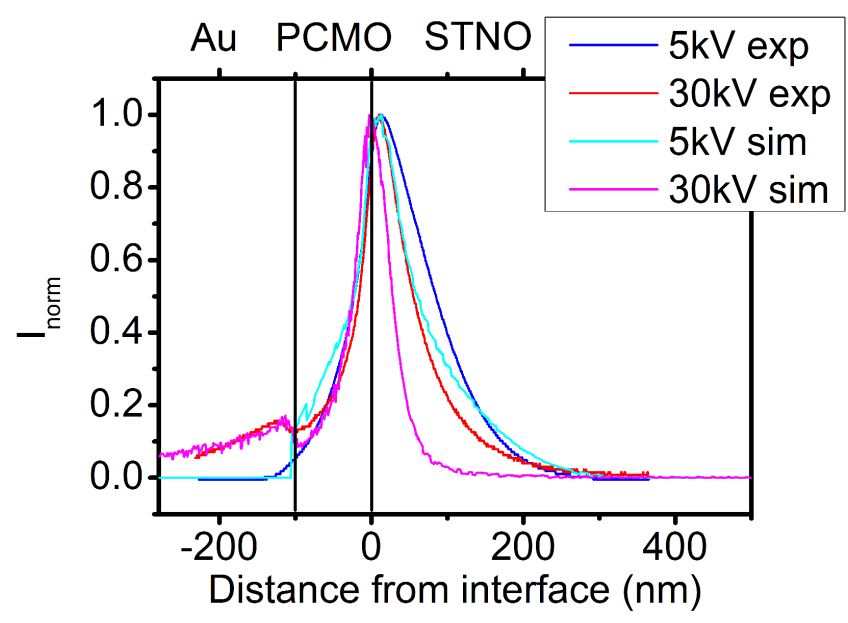

Figure 7.11: Experimental and simulation STEBIC profiles for acceleration voltages $5 \mathrm{kV}$ and $30 \mathrm{kV}$. While the general profile shape and features such as the kink at the Au-PCMO interface and the almost flat profile in the space charge region are reproduced by the simulations, the decay length does not fit for both acceleration voltages. 
Applying this model to reproduce STEBIC profiles as shown in Fig. 7.5(a) leads to results as shown in Fig. 7.11 for two different acceleration voltages. As decay lengths, we use the diffusion lengths $\mathrm{L}_{\mathrm{PCMO}}=3 \mathrm{~nm}$ and $\mathrm{L}_{\mathrm{STNO}}=17 \mathrm{~nm}$ as found in our previous work (see Ch. 6). The experimental and simulated profiles are qualitatively similar for both acceleration voltages, e.g. the pronounced kink at the Au-PCMO interface for $30 \mathrm{kV}$ is reproduced in the simulation, and is not present for $5 \mathrm{kV}$. Furthermore the broad, almost flat profile shape in the space charge region (around the maximum) and the shift of the EBIC maximum away from the p-n junction interface (at least for $5 \mathrm{kV}$ ) show up in the simulation.

Still, the simulated profiles' decay widths considerably differ from the experimental ones, especially on the STNO side. Adjusting the diffusion length as parameter in order to reach a better fit to the experimental profiles has proven impossible, as no set of diffusion lengths was found that allowed simultaneous fit at different acceleration voltages. We attribute the shift of the maximum to the slightly tilted electron beam ( 5 degrees from incidence perpendicular to the surface). It is not to be confused with the shift because of asymmetric doping found in the TEM-based STEBIC profiles (see Ch. 6).

The collection function model is thus deemed too simple to fit the experimental STEBIC data exactly, but can still serve as a guidance.

\subsubsection{COMSOL model}

Fig. 7.12 shows STEBIC profiles simulated for a point source excitation using a COMSOL model of a p-n homojunction. The first profile was simulated for parameters akin to typical Si p-n junctions. The second profile was simulated for higher doping levels, more akin to PCMO-STNO, which leads to a much more narrow profile, as can be seen by the reduced plateau width and decay length. The plateau width is corresponding to the size of the space charge region, where most excited charge carriers are collected, thus the EBIC is independent of the beam position.

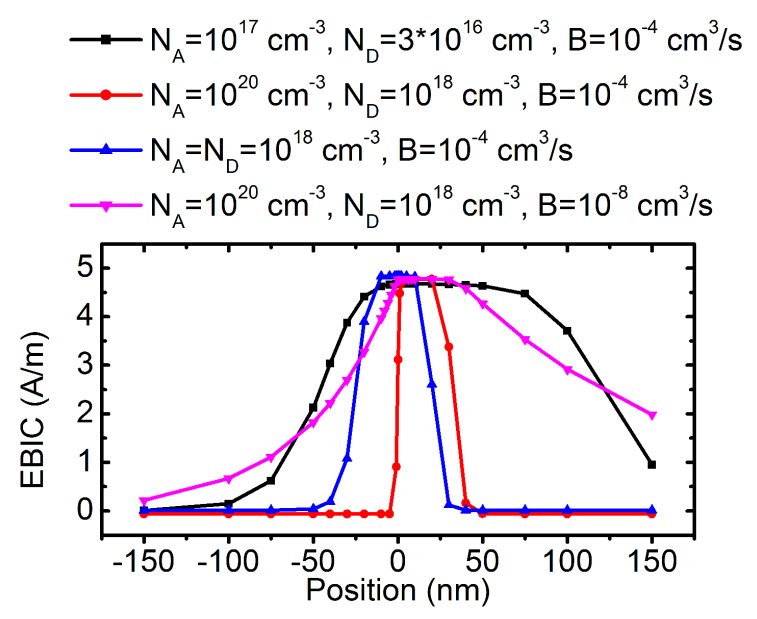

Figure 7.12: STEBIC profiles simulated by a COMSOL homojunction model for different dopings (low, high in symmetric and asymmetric ratio) and recombination coefficients, which influence the space charge region size and diffusion length. This is reflected in the plateau width and decay length of the profiles, respectively. A shift of the plateau position away from position 0 into the lower doped region for asymmetric doping is clearly exhibited, which is connected to the shift of the intrinsic point. 

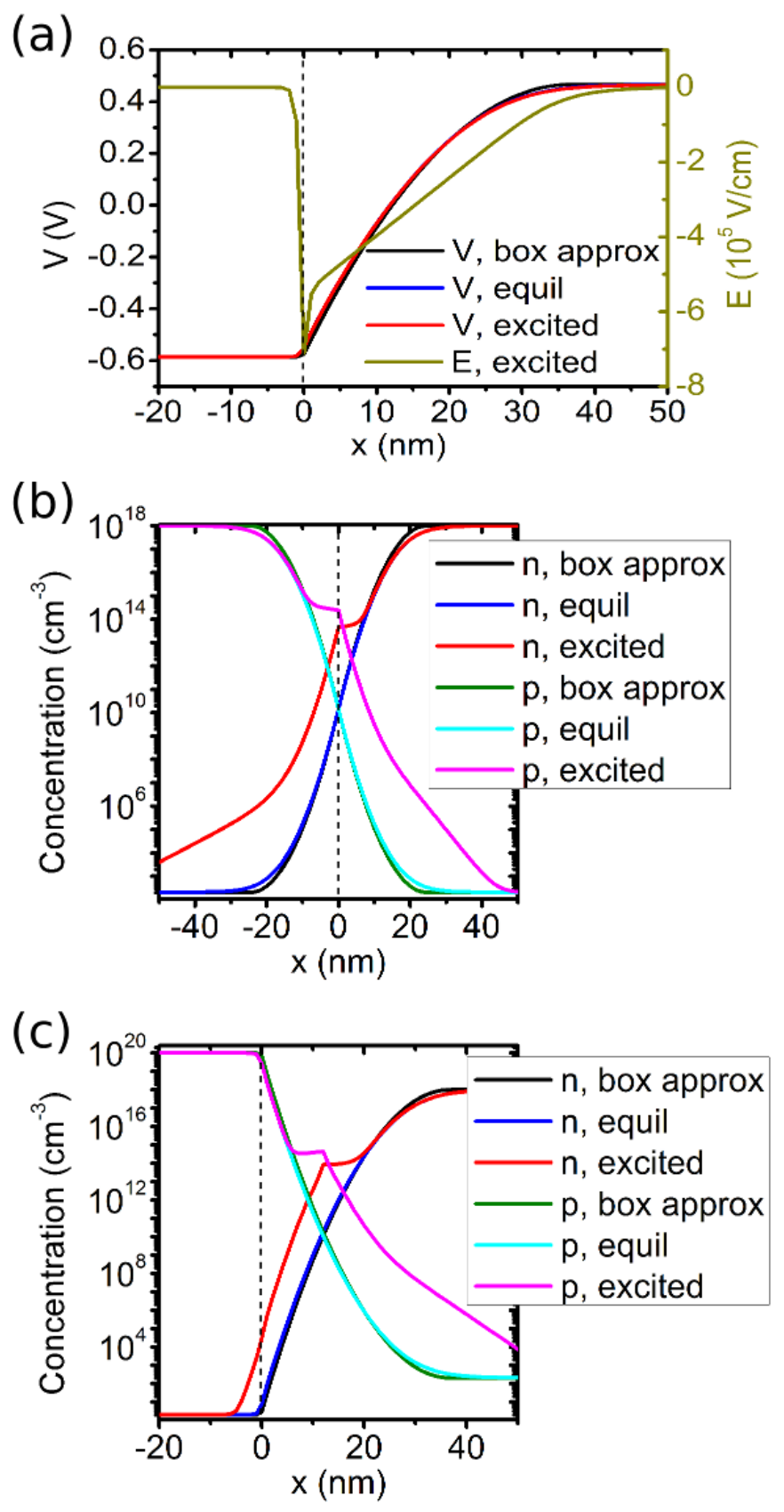

Figure 7.13: COMSOL homojunction model simulations in equilibrium and with electron beam excitation. (a) Electronic potential for asymmetric high doping, which is not greatly affected by the electron beam excitation ( $V$,equil $=V$,excited), and well described by the box approximation. The electric field maximum is at the chemical interface $(\mathrm{x}=0)$. (b) Electron and hole concentration for symmetric high doping. The maximum injection is at the intrinsic point, coincidental with the chemical interface for this doping. (c) Case for asymmetric high doping, where the maximum injection shifts with the intrinsic point into the lower doped region.

Increasing doping leads to a decreasing space charge region size, which can also be seen in our simulated profiles. The decay length is connected to the diffusion length in the neutral region, which we discussed in detail in our previous work (see Ch. 6). In general, larger diffusion length leads to larger decay length. For a constant recombination coefficient 
$B$, higher doping leads to a smaller diffusion length, as can be deduced from general recombination mechanism considerations [28]. In the case of extrinsic doping and low injection conditions, we calculate a reduction from the minority charge carrier diffusion lengths $L_{\mathrm{PCMO}} \approx \sqrt{\frac{D_{e}}{B N_{A}}} \approx 17 \mathrm{~nm}$ and $L_{\mathrm{STNO}} \approx \sqrt{\frac{D_{h}}{\mathrm{~B} N_{D}}} \approx 13 \mathrm{~nm}$ to $L_{\mathrm{PCMO}} \approx 0.5 \mathrm{~nm}$ and $L_{\mathrm{STNO}} \approx 2.3 \mathrm{~nm}$ when transitioning from low to high doping. This is in line by the reducing decay length in our simulated profiles.

The third profile was simulated for symmetric high doping, leading to a shift of the plateau region to the chemical interface $(\mathrm{x}=0)$ and a more symmetric profile. The reason for this will be further discussed in the next paragraph. The final profile was simulated for asymmetric high doping with a reduced recombination coefficient $\mathrm{B}$, leading to larger diffusion lengths $L_{\mathrm{PCMO}} \approx 53 \mathrm{~nm}$ and $L_{\mathrm{STNO}} \approx 227 \mathrm{~nm}$. This is reflected in larger decay lengths of the profile.

The shift of the STEBIC profile maximum (or plateau) into the lower doped region in case of asymmetric doping was already experimentally shown and explained in our previous publication for a PCMO-STNO heterojunction (see Ch. 6). The STEBIC profile simulations in this work confirm the doping ratio as the reason for the shift of the EBIC maximum, as was also shown for another material system 47.

We would like to elaborate further on our previously established explanation for the shift by investigating the position-dependent electronic potential $\mathrm{V}$ as well as electron and hole concentrations $n$ and $p$. Fig. 7.13(a) shows that the electronic potential is not strongly affected by the electron beam excitation, confirming the simulation is done in the low injection regime [47]. Furthermore, the maximum electric field, even in the excited case, is always at the chemical interface $(x=0)$. As shown in Fig. 7.13 (b) and (c), the point of maximum injection shifts into the lower doped region in the case of asymmetric doping. If the electron beam excites at this point, most excess charge carriers will be excited, leading to an expected maximum in the STEBIC profile. Still, the simulated STEBIC profile maximum (second profile in Fig. 7.12) is not shifted as much into the lower doped region as the intrinsic point (Fig. 7.13 (c)). This hints at an interplay of maximum injection and maximum electric field, the latter of which is located at the chemical interface regardless of the doping ratio.

\subsection{Contributions}

PCMO-STNO p-n heterojunctions were prepared by Benedikt Ifland and Christian Jooss. The COMSOL homojunction model was developed by Corin Jung and extended to a heterojunction model by the author. Holography experiments and phase reconstruction were performed by Tore Niermann. FIB preparation and EBIC experiments were performed by the author with help from Tobias Westphal. Simulations and analysis were performed by the author under guidance of Michael Seibt. Results were discussed by the author, Tore Niermann, Tobias Westphal, Tobias Meyer, Benedikt Ifland, Christian Jooss and Michael Seibt. Figures were made and text was written by the author. 



\section{Chapter 8}

\section{Discussion}

In this chapter, additional challenges of acquiring, processing and understanding the experimental data as well as conducting the simulations described in the chapters before are discussed. They will conclude in a summarizing discussion including the points already mentioned within the last chapters.

\subsection{Sample preparation}

The STEBIC lamella preparation by FIB, whether it be for SEM or for TEM based experiments, holds the distinct disadvantage that $\mathrm{Ga}$ ions are implanted into the sample lamella during the process. The implantation rate and maximum implantation depth depend on the ion beam current and voltage as well as on the material. TRIM simulations for PCMO $(x=0.35)$ processed with $30 \mathrm{kV}$ Ga ions have shown that an amorphous surface layer of about $5 \mathrm{~nm}$ thickness is formed and that the implantation depth is around $15 \mathrm{~nm}$ 151. Furthermore, STNO is subject to slightly larger damage by ion beam irradiation, with implantation depths of $36 \mathrm{~nm}$ for $30 \mathrm{kV}$ Ga ions being calculated 152. Produced damage may for example manifest in oxygen deficiency [153, 154].

The idea of using different preparation steps with decreasing ion beam currents, as has been done in this work, is to eliminate the damaged areas at the lamella surfaces. However, the safest way to avoid these layers is to only using low energy Ar ion polishing [130]. Using FIB preparation, highly damaged surface areas may still remain. In these layers, excited charge carriers recombine almost instantly [155], consequently they are called 'dead' layers [129. They have been recognized as a necessary inclusion in modeling STEBIC results in the case of low energy excitation (see Sec. 5) and are estimated to similar layer thickness for SEM as well as STEM based STEBIC experiments (see Sec. 6) on PCMO-STNO. For Si, the thickness is found to be much higher. This is understandable as amorphous surface layers due to $30 \mathrm{kV}$ Ga ion irradiation have a much larger thickness (about 20-30 nm) than in PCMO (about $5 \mathrm{~nm}$ ) 156.

In general, low energy ion polishing, e.g. by a Gatan PIPS system, is a suitable method to greatly decrease the thickness of these damaged layers, and is often used for lamellas prepared for high resolution TEM. However, this is not suitable for SEM based STEBIC samples for SEM, as the lamella is typically kept in the sample which is usually too large for the PIPS. For TEM based STEBIC lamella preparation, it is possible to transfer the DENS chips into the PIPS device, which might enable better control of dead layers in future experiments.

Aside from dead layers, FIB preparation may also produce conducting surface layers, which can for example short-circuit p-n junctions in a lamella. While diminishing the quality of STEBIC measurements, this is a desired effect for off-axis holography, as ex- 
ternal charges introduced by the electron beam can flow to the sample contacts easily, therefore they do not influence the lamella's internal electric field which is a common subject of holography studies. A similar conductive layer seems to develop due to electron beam irradiation, which was found at least in the dual beam FIB microscope during STEBIC experiments where the lamella was scanned repeatedly with dwell times of around $500 \mu \mathrm{s}$. As the electron beam cracks molecules of the remaining low pressure atmosphere in the sample chamber, such a layer is likely to form during extended irradiation. During this experiment, low energy ion beam polishing was used to remove this layer after a completed scan of the whole lamella, which worked reasonably well.

A better strategy would be to perform STEBIC experiment in better vacuum to prevent formation of the layer. Indeed, it has been found in other experiments in the NanoSEM microscope that reducing the ambient pressure from $10^{-4}$ to $10^{-6}$ mbar by using a cooling trap leads to much better stability of the surface. In situations of small STEBIC signal, the change in the STEBIC maximum can be crucial, and thus ensuring a clean surface important for reasonable results.

The preparation method for TEM based STEBIC lamellas used in this work is rather complex, including the upside down transfer to a carrier sample, subsequent macroscopic contact deposition, transfer of the contacted lamella to a DENS chip, milling of trenches to form a defined current path and final thinning. Although the STNO surface on which the back contact was deposited was only roughly cleaned by a $30 \mathrm{kV}$ ion beam, and was under normal atmosphere before transfer to the contact preparation chamber, the deposited metal layer apparently provided an Ohmic contact to STNO suitable for STEBIC measurements.

It has even been found in later experiments that it is sufficient for STEBIC measurements to deposit a Pt-C layer on STNO as back contact 157]. Thus, the contact deposition can be classified as rather uncritical. Still, the fixing of the lamella on the chip was done cautiously, restricting scanning of the electron beam to the connection points where Pt-C was deposited. This was done to prevent deterioration of the Pt contact layer on the chip where the lamella was fixed, as similar effects are known to be critical for PCMO resistance experiments [158]. After fixing the lamella, the electron beam was not reactivated for several minutes in order to let the vacuum pumps restore the normal ambient pressure and not risk deposition of residual gas on the contact layer.

\subsection{EBIC measurement}

The beam current dependent studies of the STEBIC profile maxima (Fig. 7.4(a)) could not be extended to beam currents larger than $3 \mathrm{nA}$ in the NanoLab microscope, as the measured current was, in contrary to lower currents, far off the specified value. Furthermore, the measured maximum EBIC for this beam current range was often even lower than the maxima measured at around $3 \mathrm{nA}$, which might apply for high injection conditions [52]. Additionally, it is possible that the actual ion beam current during the STEBIC measurements was lower than the value from the a posteriori Faraday cup measurement, which would mean that adjusting the electron beam in this current region cannot be trusted. Still, it has been shown that the current can be adjusted reproducably to about $7 \mathrm{nA}$ in the NanoSEM microscope 117.

During EBIC measurements with high dwell time, images may be severely impacted by drift, as well as instabilities in the microscope's dampening system, leading to jumps in 
the image (see Fig. 8.1). While it was found out that the jumps can be prevented by changing the image recording procedure to acquisition mode in the microscope software, drift always occurs. The highest drift rates $\left(1-2 \frac{\mathrm{nm}}{\mathrm{s}}\right)$ have been noticed for temperatures below $100 \mathrm{~K}$ as well as for high beam currents. While the cooling system is working almost at its limit at this temperature, which could lead to an uncontrolled drift of the stage on a small length scale, the high current induced drift is attributed to charging effects in the sample. High impinging electron currents lead to a severe local distortion of the equilibrium Coulomb potential in the sample due to charge accumulation, which may not be neutralized immediately because of limited conductance. The changed Coulomb potential may in turn affect the impinging electron beam, leading to an apparent sample drift.
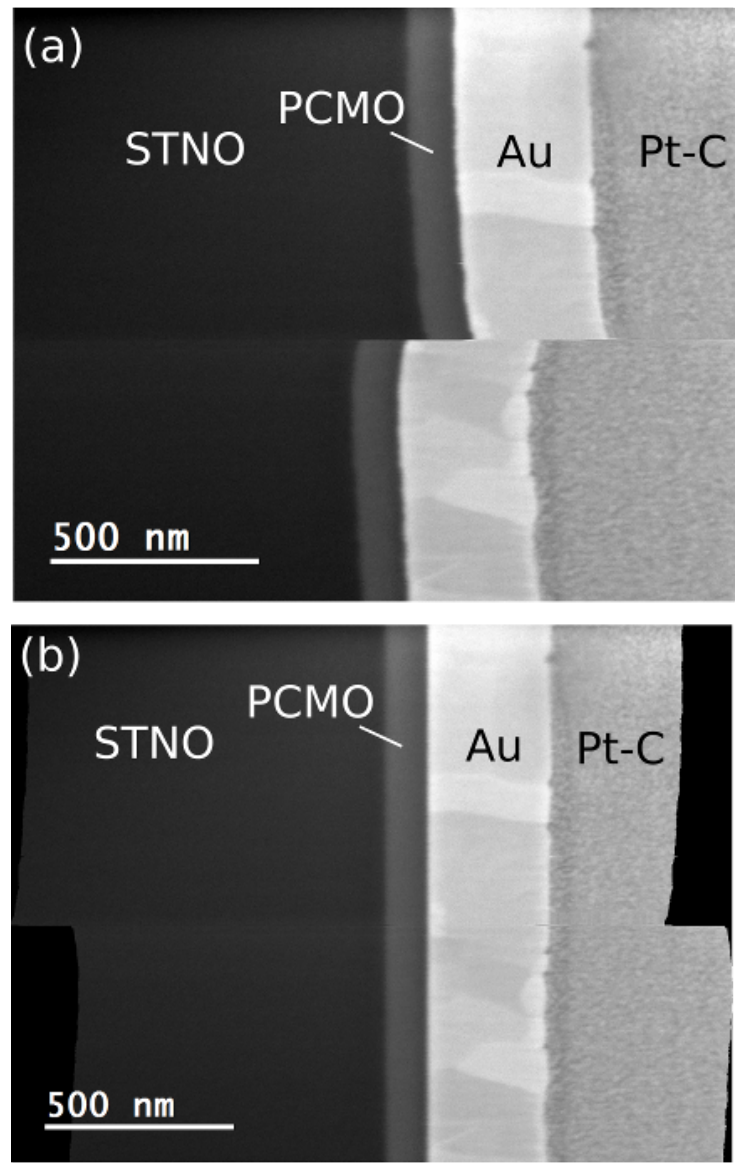

Figure 8.1: Drift correction procedure of a secondary electron image. (a) original image, showing the drift and a jump, (b) drift corrected image, also eliminating the jump. The PCMO-STNO interface was assumed as smooth on this scale in the correction procedure.

Since in situ drift correction is not implemented in the SEM control software, the images had to be drift corrected a posteriori. For this, a Gatan DigitalMicrograph script was used, comparing contrast gradients along layer interfaces visible in the secondary electron images to some reference gradient which is given by one of the first image lines. The comparison is done via cross correlation within a variable range. Running through all image lines, this procedure corrects continuous drift as well as jump-like behaviour.

To record STEBIC maps with nanometer partitioning, but still scan a whole lamella with 
about $10 \mu \mathrm{m}$ length, it is a likely strategy to perform detailed scans of individual lamella sections and stitch them together in the end. The basis for this is good drift correction, another important requirement is that clear features are visible which can be used to compare neighbouring images. The Au front contact layer has proven to be a suitable source for that, especially at its interfaces to PCMO and the protective Pt-C film on top of the lamella, which are often quite rough. A DigitalMicrograph script was used to stitch two images together by cross correlation of the parts with the same feature.

During the cooled STEBIC measurements (see Sec. 7), the temperature was measured by a temperature sensor on the cooling stage. While treated as exact in the analysis of the results, the temperature measuring is subject to errors. The measurement at the sensor can be assumed to be reasonably exact $( \pm 0.5 \mathrm{~K})$, as given by the manufacturer's description [116]. However, the sensor is not placed directly at the lamella where STEBIC measurements are conducted, consequently it may take some time for the temperature at the lamella to adjust. Assuming a given temperature at the sensor position, the time can be estimated which is necessary to adjust the lamella, connected to the bulk sample on three edges, to the same temperature, i.e. to reach thermal equilibrium with the sensor position.

The bulk sample as well as the sample holder, which mainly consists of copper and brass, should not be the limiting factor of reaching the equilibrium, as the lamella is connected to the bulk sample by much thinner interconnections than the bulk sample to the sample holder, severely limiting heat flow. The lamella also has two surfaces (beam entry and beam exit), which may emanate thermal radiation to the ambient atmosphere. As the ambient pressure is fairly low (around $10^{-5}-10^{-6} \mathrm{mbar}$ ), the temperature loss via this channel is relatively slow. The temperature adjust along the lamella was estimated by a simple analytic heat diffusion model, using the thermal diffusivity of Si and PCMO. Results indicate that the temperature adjust process happens on a timescale of some microseconds. As before each STEBIC measurement, it was waited for several minutes after the indicated temperature had stabilized, the temperature should be safely equalized at the lamella and the temperature point. Nevertheless, a temperature difference from the temperature sensor to the lamella cannot be excluded.

\subsection{EBIC processing}

The EBIC image quantification procedure, as described in Sec. 3.9.1, was used on all analyzed data in this work in order to transfer the quantitative measurement from the measurement board to the simultaneously recorded qualitative EBIC images. Two factors are critical in the procedure: Choosing the correct background signal in the quantitative measurement and choosing reasonable quantiles to match the cumulative histograms of the quantitative to the qualitative data.

The background signal is collected with a blanked electron beam, so it should give a good reference of where the STEBIC signal is zero. However, in practice this reference is not always reliable, as it is sometimes higher than some signal taken during the electron beam scan, i.e. would produce negative EBIC signal when used as a reference. Furthermore, it may be so small that the signal from the STEBIC profile does not saturate around zero, but at some higher value. Both effects may be explained by a change of the reference during the STEBIC measurement, i.e. by charging effects in case of poor contacting. The different background levels in PCMO and STNO, as well as in p-doped and n-doped 
Si can be explained by taking into account Eq. 2.17. If the bulk charge carrier concentration, $p_{e q}$ in the p-doped and $n_{e q}$ in the n-doped area, is different, i.e. asymmetric doping of the p-n junction, the injection level on both sides far away from the junction interface $\delta p$ also differs. The effect in experimental signal as well as calculated injection is shown in Fig. 8.2 .

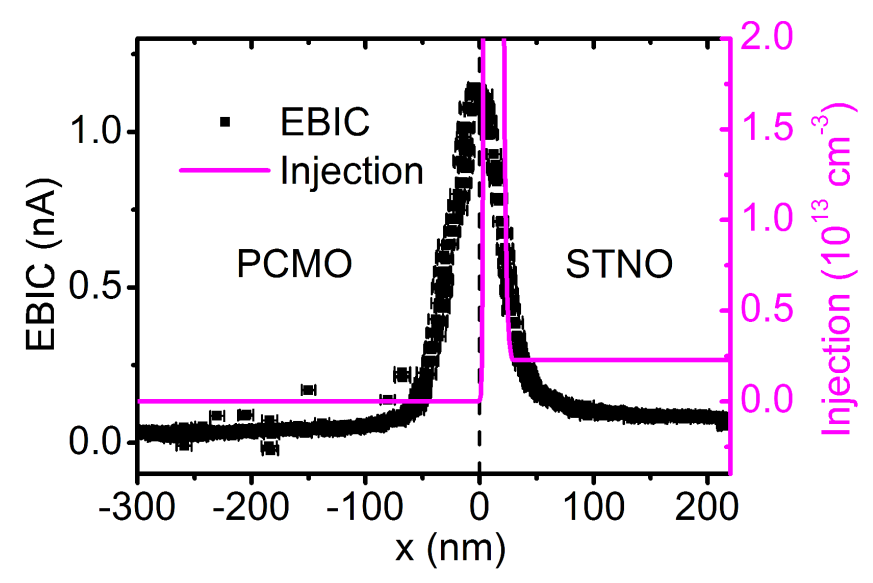

Figure 8.2: $80 \mathrm{kV}$ STEBIC profile, showing the slightly differing saturation levels in PCMO and STNO far away from the p-n junction interface. Also plotted is the local injection level (cropped at the top), as calculated from Eq. 2.17. It shows the same qualitative relation of saturation levels as the STEBIC profile, indicating that the different levels are primarily due to the asymmetric doping levels of the materials.

Choosing meaningful quantiles is important to impose the correct scaling to the qualitative data. The quantiles were mostly chosen near the lower and upper end of the histogram, as shown in Fig. 8.3(a), and compared if they correspond to the same quantiles in the histogram of the quantitative and qualitative data when the quantitative scale is imposed on the qualitative data. The error of this procedure is estimated to be about $2 \%$, which was taken into account deriving the errors of EBIC maxima.

A new version of the processing script has been developed, using cross correlation to compare the histograms of the quantitative and qualitative measurements and extracting the background from a scan interruption marker in the measurement file. The results from processing with the script used in this work and the newer one are comparable, as shown in Fig. 8.3(b), but choosing the newer script is preferable for future experiments as it does not depend on the user choosing quantiles manually and the cross correlation is more robust than comparing just two points in the histogram.

\subsection{Correction of EBIC preamplifier limitations}

The bandwidth limitations of the Stanford Research SR570 preamplifier were explained in Sec. 3.4.1. For high amplifier sensitivity settings, the bandwidth is severely reduced, which makes high dwell times during recording necessary. In fact, at both used scanning electron microscopes used in this work, the dwell time cannot be set higher than $1 \mathrm{~ms}$ (for the Nanolab FIB) and $10 \mathrm{~ms}$ (for the NanoSEM), rendering sensitivity settings of the amplifier in the $\mathrm{pA} / \mathrm{V}$ range useless. Still, in the Titan TEM the dwell time is more variable, thus an EBIC setup at this microscope including the amplifier may still profit 

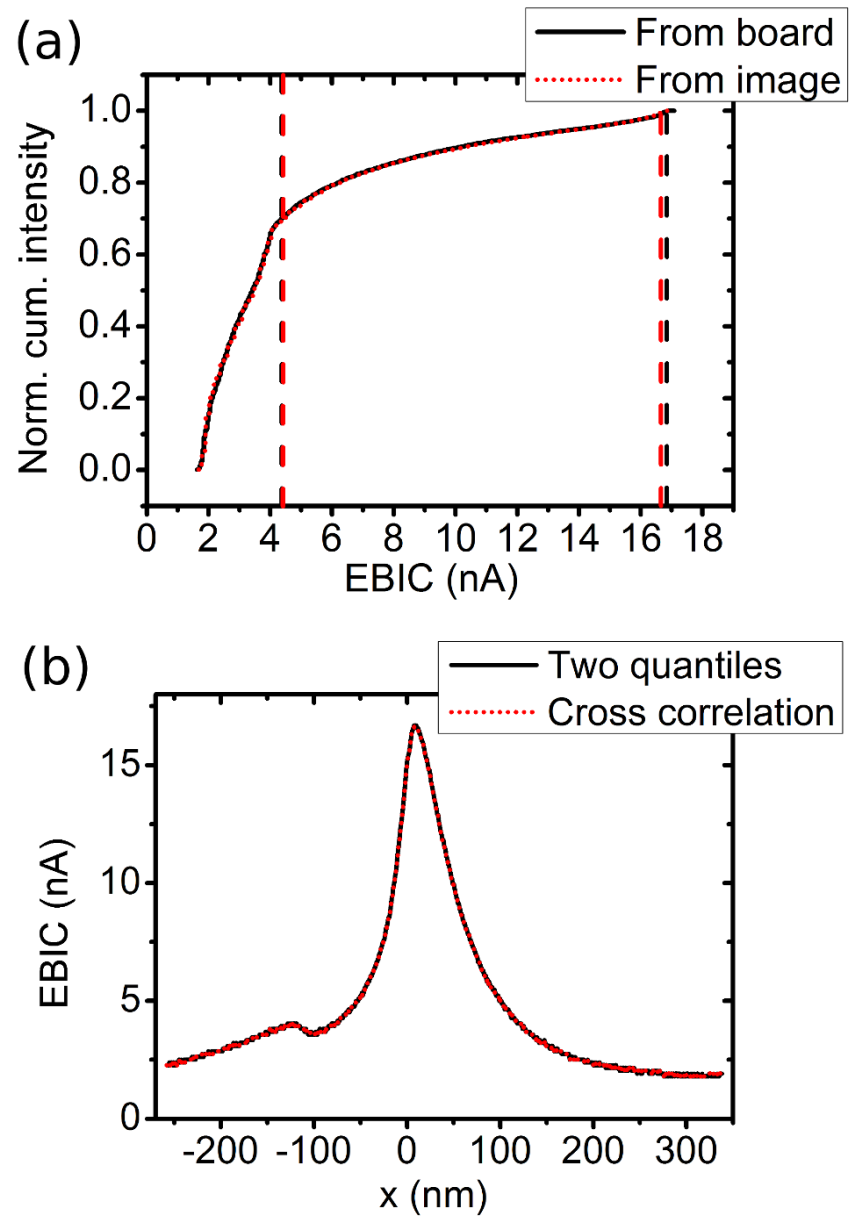

Figure 8.3: Processing of $30 \mathrm{kV}$ STEBIC profiles. (a) shows typical cumulative histograms, normalized to their saturation value. The histogram of the EBIC quantitative measurement, taken by the measurement board, is compared to the histogram of the qualitative EBIC image, and the current scale (abscissa) of the quantitative measurement is superimposed by comparing the cumulated values at two quantiles (here $70 \%$ and $99.8 \%$ ). (b) Typical resulting STEBIC profile. The processing method using the two quantiles is compared to a newer script using cross correlation of quantitative and qualitative histogram. The results of the old and the new method do not show significant deviations.

from the high sensitivity settings. Still, even if the bandwidth limitation is accounted for, EBIC signal is still overall reduced for high amplifier sensitivies, which can be corrected by multiplying a factor to the EBIC profile or map. However, the exact value of the factor was found to be dependent on the amplifier sensitivity and the sample.

In the following, a simple model will be described to connect the correction factors to the amplifier input resistance. It is based on the one diode model Shockley equation, which is given in equation 2.37 in chapter 2. For EBIC measurements, no voltage is applied, thus we can set $U=0$. Furthermore, in the experimental results shown in Fig. 3.10. the absolute EBIC signal is always below $I=250 \mathrm{nA}$ (the used beam currents were $I_{b}=0.47 \mathrm{nA}$ for $5 \mathrm{kV}$ cross section, $0.7 \mathrm{nA}$ for $15 \mathrm{kV}$ cross section and $0.27 \mathrm{nA}$ for $5 \mathrm{kV}$ top view measurements). Assuming a safe upper limit for the series resistance such as 


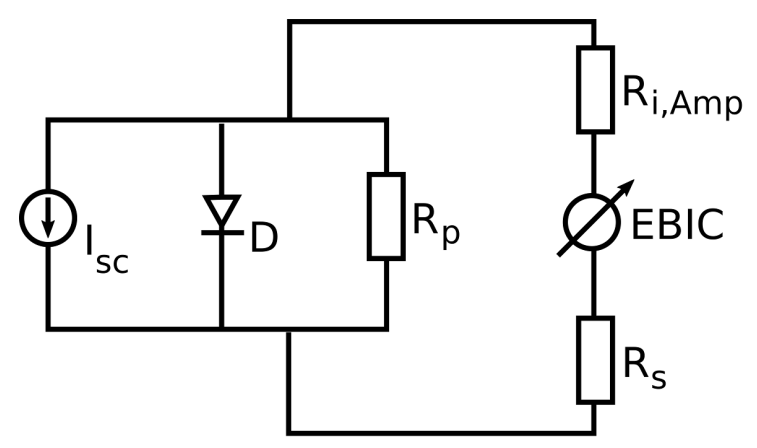

Figure 8.4: Equivalent circuit for an EBIC measurement in a one diode model with ideality factor $n=1$. Compared to the usual one diode model as shown in Fig. 2.4, the voltage source is replaced by an amperemeter to measure the EBIC. The used current preamplifier uses a variable input resistance $R_{i, A m p}$, represented here by an additional resistor.

$R_{s}=1 \mathrm{k} \Omega$, a reasonable ideality factor $n \geq 1$ and $T=300 \mathrm{~K}$, we find that

$$
I \cdot R_{s} \ll \frac{n k_{B} T}{e} .
$$

In this approximation, we may set the exponential from Eq. 2.37 to 1, thus simplifying it to

$$
I \approx \frac{-I_{s c}}{1+\frac{R_{s}}{R_{p}}}
$$

In Fig. 8.4, the equivalent circuit for an EBIC measurement is sketched. The preamplifier is inserted in series between the sample and the actual current measurement, represented by an amperemeter in the equivalent circuit. Thus we may add the amplifier input resistor with resistance $R_{i, A m p}$ in series to the series resistor with resistance $R_{s}$. Consequently, the input resistance shows up in the simplified Shockley equation as an additional series resistance. In this model, the ratio between the short circuit current, which is the actual EBIC, and the measured EBIC is

$$
\left|\frac{I_{s c}}{I}\right| \approx 1+\frac{R_{s}+R_{i, A m p}}{R_{p}}=: \alpha_{i} .
$$

This is the proposed simple model for a correction factor $\alpha_{i}$ for high sensitivity EBIC measurements, where the high input resistance comes into play. It requires knowledge of $R_{s}$ and $R_{p}$. According to the Shockley theory, the inverse slope of $I(U)$ is $R_{s}$ for sufficiently large forward bias $(U>0)$ and $R_{s}+R_{p}$ for sufficiently large reverse bias $(U<0)$. Before and after the SEM-based EBIC measurements in this work, the inverse slope was routinely checked in both directions by a multimeter. The measurement of the sample used for cross section measurements in Fig. 3.10 was checked by extracting the resistances from the slope of the recorded $I(U)$ curve, which is shown in Fig. 7.3 in chapter 7. The results for both methods and samples are shown in Table 8.1. Whereas $R_{p}$ is comparable to the value extracted with the multimeter method, $R_{s}$ is considerably smaller. The multimeter applies a small voltage in order to measure a sample resistance, which may be the reason for the altered series resistance. Thus, the values of the $I(U)$ method are more trustworthy.

The determined correction factors for the cross section measurement, obtained from the 


\begin{tabular}{l||c|c|c|c} 
Sample, Method & $R_{s}(\Omega)$ & $R_{p}(\Omega)$ & $\alpha_{i}, R_{i, a m p}=100 \Omega$ & $\alpha_{i}, R_{i, a m p}=10 \mathrm{k} \Omega$ \\
\hline Cross Section, Multimeter & 900 & 3000 & 1.33 & 4.63 \\
Cross Section, $I(U)$ & $13.79(2)$ & $3313(3)$ & 1.03 & 4.02 \\
Top View, Multimeter & 700 & 1600 & 1.50 & 7.69
\end{tabular}

Table 8.1: Results of the proposed simple EBIC correction procedure for high amplifier sensitivity

$I(U)$ method, are very close to the values found by comparing the experimental EBIC profiles. Furthermore, as $\alpha_{i} \approx 1$ already for $R_{i, a m p}=100 \Omega$, it can be concluded that the EBIC values obtained from the low sensitivity range, i.e. up to $200 \mathrm{nA} / \mathrm{V}$, are the real quantitative EBIC values. To also obtain these in the high sensitivity range, $\alpha_{i}$ for $R_{i, a m p}=10 \mathrm{k} \Omega$ must be multiplied to the EBIC results from this range. The correction factor found for the top view measurements is also close to the result from comparing the low and high sensitivity EBIC profiles. Unfortunately, no $I(U)$ measurement is available for this sample, which is supposed to be able to provide a better estimation for $\alpha_{i}$.

In conclusion, to estimate this factor properly, it is recommended to record an $I(U)$ curve for the used sample, preferably within the setup actually used for EBIC measurements in order to estimate the series resistance correctly. The larger the parallel resistance of the sample, i.e. the closer it is to an ideal diode, the smaller the required correction factor. The proposed simple correction procedure is valid for any type of EBIC sample, as long as voltage drop over the series resistance is not too high (usually, the current should be below at least $10 \mu \mathrm{A}$ at room temperature).

Determined correction factors can be applied in order to estimate the electron hole pair excitation energy $E_{e h}$, as defined in Eq. 2.1. In the modeling of a STEBIC profile by convolution of a simulated generation volume and a given collection function, one may write Eq. 2.41 in one dimension with a proportionality factor including $E_{e h}$ :

$$
I_{\text {sim }}\left(x_{0}\right)=\frac{I_{b}}{E_{e h}} \Sigma \varepsilon_{a}=\frac{I_{b}}{E_{e h}} \int_{x} \varepsilon_{a}\left(x-x_{0}\right) \cdot f(x) \mathrm{d} x
$$

with the beam position $x_{0}$, beam current $I_{b}$, cumulated absorbed energy $\Sigma \varepsilon_{a}$, local absorbed energy $\varepsilon_{a}$ and collection function $f(x)$, as defined in Eq. 2.40. Comparing simulated and experimental STEBIC profiles, as shown in Fig. 7.11, offers the possibility to compare the simulated $\Sigma \varepsilon_{a}$ to the experimental STEBIC signal, for example at the distinct point of the maximum. Using the correction factor $\alpha_{i}$ for the given experimental conditions, the experimental and simulated maxima may be connected by $I_{\max , \operatorname{sim}}=$ $\alpha_{i} \cdot I_{\text {max }, \text { exp }}$. Therefore $E_{e h}$ can be determined by

$$
E_{e h}=\frac{I_{b} \cdot\left(\Sigma \varepsilon_{a}\right)_{\max }}{\alpha_{i} \cdot I_{\max , \exp }}
$$

Using the data from Fig. 7.4(a) and 7.11 for 5 and $30 \mathrm{kV}$, a first attempt of determining $E_{e h}$ was taken as shown in Tab. 8.2 . The dwell time was high enough $(500 \mu \mathrm{s})$ to exclude bandwidth limitations. $R_{s}$ could not be measured reliably for these data, therefore it was assumed that $R_{s} \ll R_{i, A m p}$, meaning that $R_{s}$ can be neglected to calculate $\alpha_{i}$ based on Eq. 8.1. For these conditions, $\alpha_{i} \approx 2$ was calculated for both measurements.

The resulting electron hole pair energy is roughly equal for both measurements, and, using the Eq. 2.1. can be converted to a band gap energy of $E_{g} \approx 3.5-5 \mathrm{eV}$ (see 


\begin{tabular}{r||c|c|c|c} 
Acceleration voltage & $I_{b}(\mathrm{nA})$ & $\left(\Sigma \varepsilon_{a}\right)_{\max }(\mathrm{keV})$ & $I_{\max , \exp }(\mathrm{nA})$ & $E_{e h}(\mathrm{eV})$ \\
\hline $5 \mathrm{kV}$ & 1.55 & 1.33 & $76(1)$ & $14(1)$ \\
$30 \mathrm{kV}$ & 0.61 & 0.54 & $15(1)$ & $11(1)$
\end{tabular}

Table 8.2: Calculation of electron hole pair energy by comparing experimental and simulated data using the correction factor $\alpha_{i} \approx 2$ for high amplifier sensitivity.

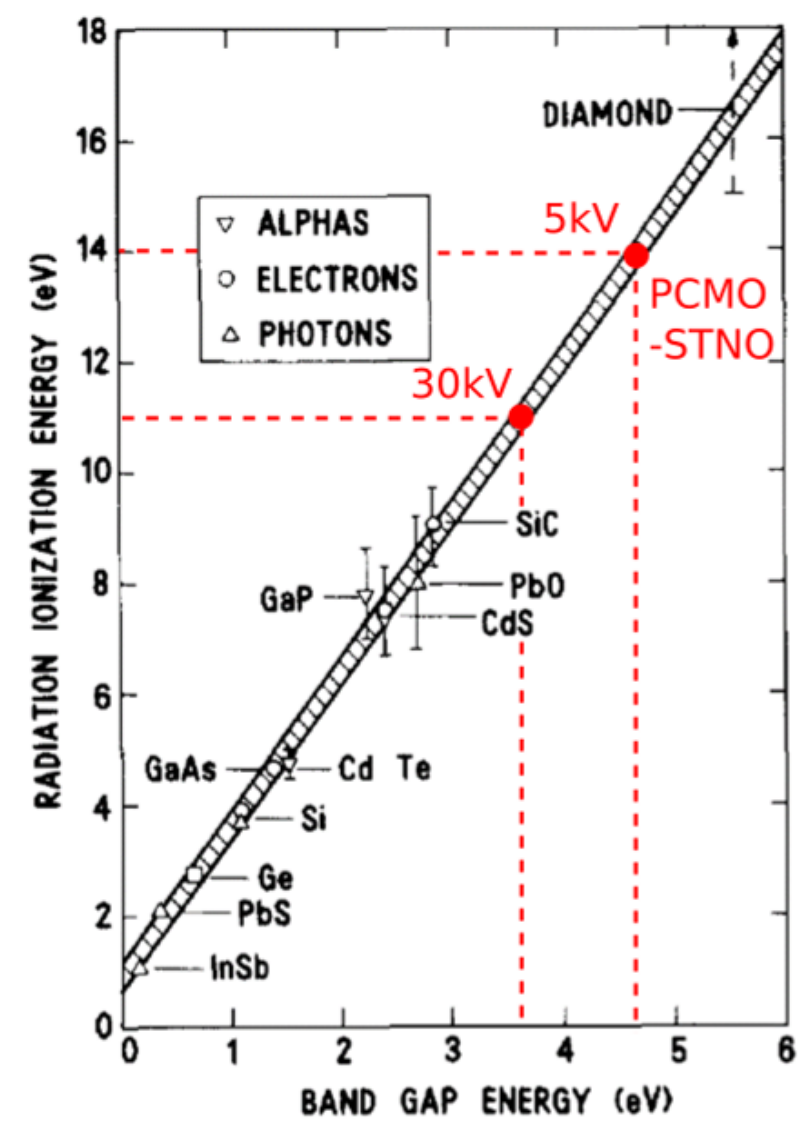

Figure 8.5: Calculated electron hole pair energies written into the universal diagram shown by Klein 25] (picture taken from [17]), showing the corresponding bandgaps in the range of $3.5-5 \mathrm{eV}$, which is close to the STNO bandgap and within the spectral range where PCMO absorption is highest.

Fig. 8.5). This is comparable to the STNO bandgap $(3.2 \mathrm{eV})$ and within the range of absorbed energy in PCMO, as shown in Fig. 2.13(b). If the collection function model can be improved, for example to incorporate surface effects, this method may provide a good strategy of estimating a mean energy characteristic for the excess charge carrier excitation process.

\subsection{CASINO simulation}

CASINO simulations are performed with a large number of electrons in order to ensure good statistics in the spatial distribution of deposited energy. It has been found that repeating the simulation does not noticeably change the resulting STEBIC profile simu- 
lations when choosing at least $2 \cdot 10^{5}$ simulated electrons. This is in spite of the fact that repeating CASINO v2 simulations with the same parameters often leads to a changed total volume in which the spatial energy distribution is calculated.

Particularly, the volume extension in the plane parallel to the beam entry surface often changes by several $100 \%$ from one simulation to another. Judging from the description [107], it should be calculated from the electron range (after [159]), which is constant for the same material and presets. However, it seems the program does not simply use this value, but calculates the plane extension from arbitrarily changing parameters. It can be speculated that it uses the actual range of the first simulated impinging electron. This bug does not strongly influence the STEBIC profile simulations, but prevents a robust estimate of the lateral extension of the generation volume (shown in Fig. 6.1). Therefore, for these simulations CASINO v3 was used, which includes an adjustable extension of the volume for which to simulate the deposited energy. This version, however, is unsuitable to simulate multilayer samples, as a bug seems to prevent realistic electron transfer between layers. Therefore, both CASINO versions have their distinct use, depending on the application of the simulation.

One of the main limitations of the collection function model (Eq. 7.12), as well as of an accurate COMSOL model, is the need to simulate the generation volume for each beam position, as the chemical PCMO-STNO interface moves in relation to the beam, and the energy distribution differs from PCMO to STNO because of their different mass density (see Fig. 8.6(a)). A more distinct change can also be seen when transitioning from PCMO to the Au front contact (see Fig. 8.6(b)). The transition from Au to PCMO is responsible for the kink in the STEBIC profile at their interface. Consequently, this is not visible when assuming one constant generation volume for all beam positions, see Fig. 8.6(c). However, in the PCMO and STNO region, simulated profiles for many simulated generation volumes and one generation volume (which was taken from the beam position at the PCMO-STNO interface) are almost identical.

In conclusion, for further studies using the collection function model, it is convenient to use only one generation volume, significantly reducing simulation effort. It is also reasonable to transfer this finding to COMSOL simulations.

\subsection{COMSOL simulation}

Instead of the used Neumann (i.e. flux) boundary conditions used at the sample surfaces in the final simulations (see Ch. 7) for the continutity equations (Eq. 2.2 and 2.3), it is also possible to use Dirichlet boundary conditions, which however imply that no current flows through the surfaces. This is not reasonable for the contacts, but may be for the beam entry and beam exit surfaces. Conditions of no current flux are equal to zero surface recombination velocity, which may be a good approximation if the velocity in the flux condition is very small, e.g. $s<10^{3} \frac{\mathrm{cm}}{\mathrm{s}}$.

Furthermore, choosing a fixed potential at the surfaces, for example in the center of the band gap, would imply Fermi level pinning due to a high concentration of charged defects. This would also imply an electric field at the surfaces, additionally acting as a charge carrier source or sink. So far, no stable conditions in the simulations could be reached using the described conditions.

Additionally to the homojunction COMSOL model described in Ch. 7, a heterojunction model was implemented, based upon the model developed in Sec. 2.1.5. Similarly to 

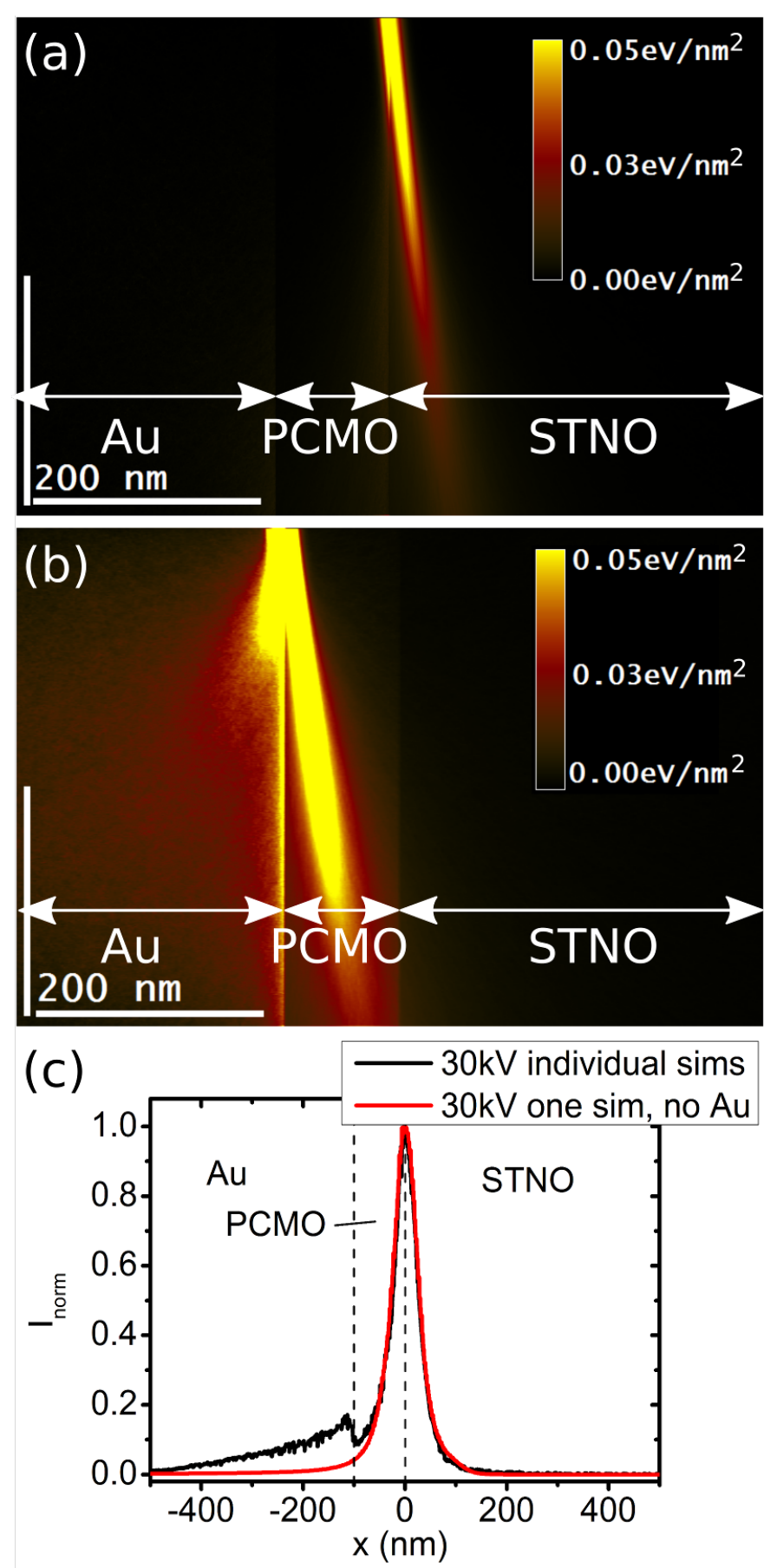

Figure 8.6: Simulated generation volume. The $30 \mathrm{kV}$ beam impinges the sample at (a) PCMOSTNO, (b) Au-PCMO interface. (c) Simulated STEBIC profile using an adjusted generation volume for each beam position (individual sims) and the same generation volume, as shown in (a), for each beam position (one sim). For the latter, the Au layer was left out of the simulation. Aside from the kink at the Au-PCMO interface, the simulated profiles match.

the homojunction model, two simulation steps are used. In the first one, the Poisson- 
Boltzmann equation

$$
-\frac{\mathrm{d}}{\mathrm{d} x}\left(\varepsilon \frac{\mathrm{d} V}{\mathrm{~d} x}\right)=e \cdot\left(p_{0, W} \exp \left(\frac{-e V}{k_{B} T}\right)-n_{0, W} \exp \left(\frac{e V}{k_{B} T}\right)+C_{\text {ion }}\right)
$$

is solved in a one dimensional sample geometry. In the second one, the set of Poisson and charge carrier equations

$$
\begin{aligned}
-\vec{\nabla}(\varepsilon \vec{\nabla} V) & =e \cdot\left(p-n+C_{\text {ion }}\right) \\
\vec{\nabla} \cdot\left(-D_{p} \vec{\nabla} p-\mu_{p} p \vec{\nabla} V+D_{p} \frac{p}{p_{0, W}} \vec{\nabla} p_{0, W}\right) & =\frac{G}{E_{G}}-B \cdot\left(n p-n_{i}^{2}\right) \\
\vec{\nabla} \cdot\left(-D_{n} \vec{\nabla} n+\mu_{n} n \vec{\nabla} V+D_{n} \frac{n}{n_{0, W}} \vec{\nabla} n_{0, W}\right) & =\frac{G}{E_{G}}-B \cdot\left(n p-n_{i}^{2}\right)
\end{aligned}
$$

is solved in the two dimensional geometry with electron beam excitation, using the results from the first simulation step as initial values. The electron beam excitation is still implemented as a stripe source, and boundary conditions for the charge carrier concentrations are modified to

$$
\begin{aligned}
\vec{\eta} \cdot\left(-D_{p} \vec{\nabla} p-\mu_{p} p \vec{\nabla} V+D_{p} \frac{p}{p_{0, W}} \vec{\nabla} p_{0, W}\right) & =S \cdot \delta p \\
\vec{\eta} \cdot\left(-D_{n} \vec{\nabla} n+\mu_{n} n \vec{\nabla} V+D_{n} \frac{n}{n_{0, W}} \vec{\nabla} n_{0, W}\right) & =S \cdot \delta n
\end{aligned}
$$

The used parameters are collected in Table 8.3. A smaller sample size is chosen in order to better account for the small dimensions in which the potential changes are happening in PCMO-STNO, compared to the Si homojunction model. Permittivity, mobility, diffusion constants and bandgap have been adjusted to the known values of PCMO-STNO. The diffusion potential $V_{D}$ is calculated from the known work functions $W_{p}$ and $W_{n}$ of the materials. The nominal doping concentrations of PCMO-STNO are $N_{A, \text { nom }}=6.3 \cdot 10^{21} \mathrm{~cm}^{-3}$ and $N_{D, n o m}=3 \cdot 10^{19} \mathrm{~cm}^{-3}$. However, simulations have proven more stable using concentrations reduced by about one order of magnitude: $N_{A, \text { red }}=$ $10^{20} \mathrm{~cm}^{-3}$ and $N_{D, \text { red }}=10^{18} \mathrm{~cm}^{-3}$, equal to the concentration used for the highly doped Si homojunction model. Using the given $V_{D}, N_{A}$ and $N_{D}$, the intrinsic charge carrier concentration may be calculated for a homojunction by

$$
n_{i}=\sqrt{N_{A} N_{D} \exp \left(-\frac{e V_{D}}{k_{B} T}\right)} .
$$

For heterojunctions, $n_{i, p}$ and $n_{i, n}$ are generally different on both junction sides, and equation 8.10 becomes more complex, including the bandgaps, electron affinities and effective densities of states of both materials 144. As many of these parameters are unknown for PCMO and STNO, the result from Eq. 8.10 is at first used as an approximation for both materials. It was estimated that this approximation provides an upper limit for the real intrinsic charge carrier concentrations for both materials.

Using the approximations from Eq. 2.24 and 2.25, the used recombination coefficient $B=10^{-10} \frac{\mathrm{cm}^{3}}{\mathrm{~s}}$ corresponds to bulk minority charge carrier diffusion lengths of $L_{\mathrm{PCMO}} \approx$ $2 \mathrm{~nm}$ and $L_{\mathrm{STNO}}=161 \mathrm{~nm}$ at the reduced doping concentration and to $L_{\mathrm{PCMO}} \approx 0.3 \mathrm{~nm}$ and $L_{\mathrm{STNO}} \approx 29 \mathrm{~nm}$ at the nominal doping concentration. The material jump in several constants was realized as a weakened Heaviside function, with a transition region size of 


\begin{tabular}{l|c|c|c} 
Parameter & Symbol & Value p area & Value n area \\
\hline Temperature & $T$ & $300 \mathrm{~K}$ & $300 \mathrm{~K}$ \\
Length & $l_{p}, l_{n}$ & $20 \mathrm{~nm}$ & $200 \mathrm{~nm}$ \\
Thickness & $t$ & $50 \mathrm{~nm}$ & $50 \mathrm{~nm}$ \\
Permittivity & $\varepsilon$ & $30 \varepsilon_{0}$ & $306 \varepsilon_{0}$ \\
Hole mobility & $\mu_{p}$ & $0.02 \mathrm{~cm}^{2} /(\mathrm{V} \cdot \mathrm{s})$ & $1 \mathrm{~cm}^{2} /(\mathrm{V} \cdot \mathrm{s})$ \\
Electron mobility & $\mu_{n}$ & $0.02 \mathrm{~cm}^{2} /(\mathrm{V} \cdot \mathrm{s})$ & $1 \mathrm{~cm}^{2} /(\mathrm{V} \cdot \mathrm{s})$ \\
Hole diffusion constant & $D_{p}$ & $k_{B} T \mu_{p} / e$ & $k_{B} T \mu_{p} / e$ \\
Electron diffusion constant & $D_{n}$ & $k_{B} T \mu_{n} / e$ & $k_{B} T \mu_{n} / e$ \\
Generation rate & $G_{p n}$ & $10^{30} \mathrm{eV} /\left(\mathrm{cm}^{3} \cdot \mathrm{s}\right)$ & $10^{30} \mathrm{eV} /(\mathrm{cm} \cdot \mathrm{s})$ \\
Bandgap & $E_{G}$ & $2.5 \mathrm{eV}$ & $3.2 \mathrm{eV}$ \\
Recombination coefficient & $\mathrm{B}$ & $10^{-10} \mathrm{~cm} / \mathrm{s}$ & $10^{-10} \mathrm{~cm} / \mathrm{s}$ \\
Contact recombination velocity & $S_{c o n t}$ & $10^{8} \mathrm{~cm} / \mathrm{s}$ & $10^{8} \mathrm{~cm} / \mathrm{s}$ \\
Surface recombination velocity & $S_{s u r f}$ & $10^{5} \mathrm{~cm} / \mathrm{s}$ & $10^{5} \mathrm{~cm} / \mathrm{s}$ \\
Bulk hole concentration & $p_{0}$ & $N_{A}$ & $n_{i}^{2} / N_{D}$ \\
Bulk electron concentration & $n_{0}$ & $n_{i}^{2} / N_{A}$ & $N_{D}$ \\
Work function & $W$ & $4.9 \mathrm{eV}$ & $4.1 \mathrm{eV}$ \\
Potential offset & $V_{0}$ & 0 & 0 \\
Diffusion potential & $V_{D}$ & $\left(W_{p}-W_{n}\right) / e$ & $\left(W_{p}-W_{n}\right) / e$ \\
Hole concentration constant & $p_{0, W}$ & $N_{A}$ & $n_{i}^{2} / N_{D} \cdot \exp \left(\frac{e V_{D}}{k_{B} T}\right)$ \\
Electron concentration constant & $n_{0, W}$ & $\frac{n_{i, p}^{2}}{N_{A}}$ & $N_{D} \cdot \exp \left(\frac{-e V_{D}}{k_{B} T}\right)$ \\
& &
\end{tabular}

Table 8.3: Parameters used in COMSOL heterojunction model

$1 \mathrm{~nm}$.

Fig. 8.7 shows results from the PCMO-STNO heterojunction model for the reduced and the nominal doping concentration, with electron beam excitation at the intrinsic point. The equilibrium solution provides insight into the shift of the intrinsic point and the homojunction model for similar doping, approaching the PCMO-STNO heterojunction model. Even though the doping levels are extremely high in the materials, the box approximation still approximately holds, as the COMSOL equilibrium solution for the electronic potential as well as electron and hole concentrations only slightly differ from the box approximation results. The electric field increase with increasing doping concentration is also shown. The excited solution of the potential does not differ significantly from the equilibrium solution, whereas the charge carrier concentration peak at the excitation point and decay across the whole space charge region in the excited solution.

This is all very similar to the results from the homojunction model. However, as expected, the concentrations show a jump at the chemical interface. This jump may change direction with changing intrinsic charge carrier concentration, as shown in Fig. 8.7(c). Aside from the jump, a peak in these results at $x=0$. As of now, the origin of these peaks is not clear. It may be the result of numerical problems, connected to the jump of the constants $p_{0, W}$ and $n_{0, W}$ at this point, which does not occur for identical intrinsic charge carrier concentration on both sides. On the other hand, the occurrence of locally increased charge carrier concentration is a known feature of heterojunctions [154]. If this is happening in PCMO-STNO can only be answered by more sophisticated simulations, but STEBIC experiments have, so far, not given any indication of such an effect. 

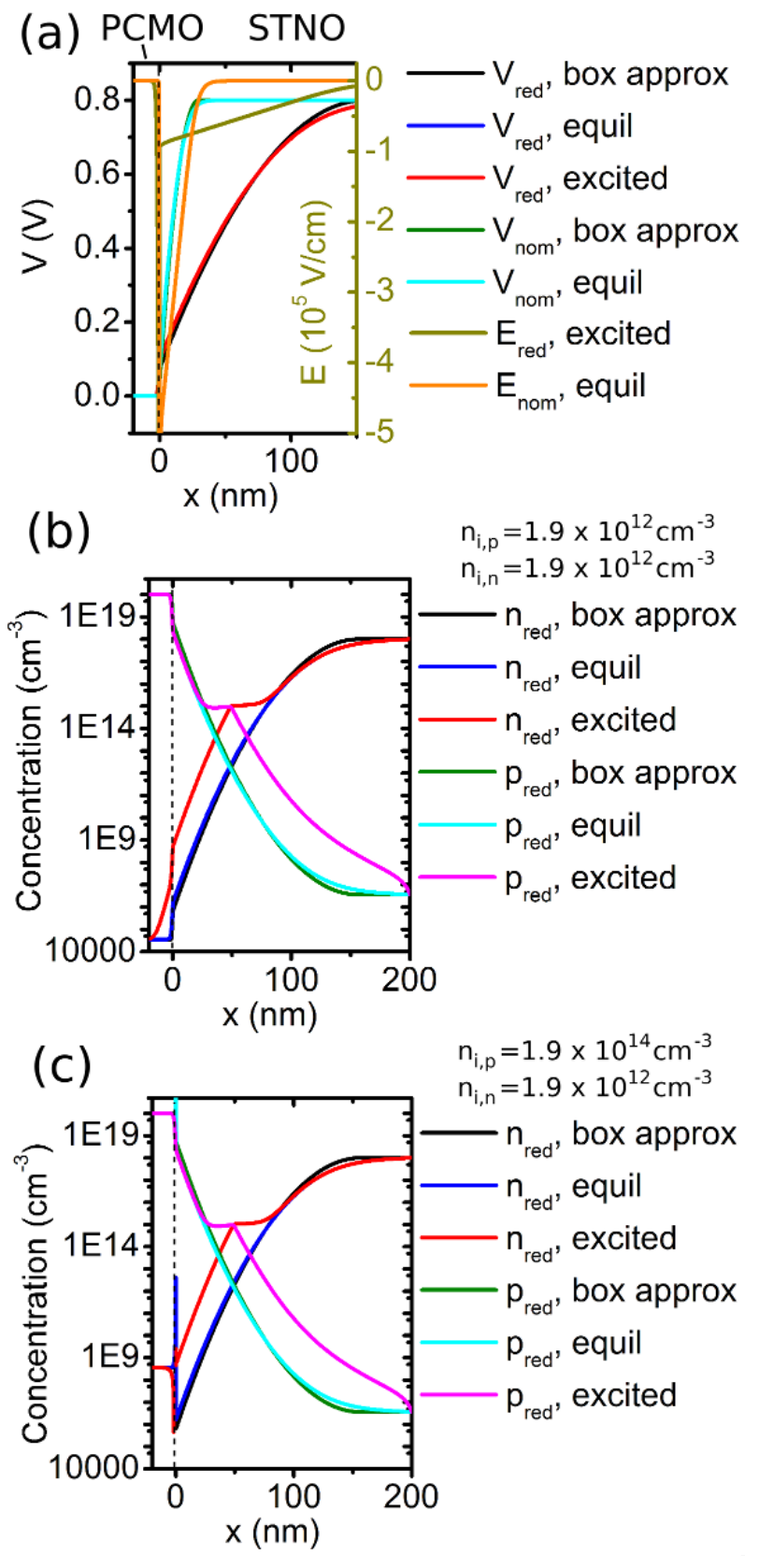

Figure 8.7: COMSOL PCMO-STNO heterojunction model. (a) Electronic potential and field for reduced doping $\left(N_{A}=10^{20} \mathrm{~cm}^{-3}, N_{D}=10^{18} \mathrm{~cm}^{-3}\right)$ and nominal doping $\left(N_{A}=6.3\right.$. $10^{21} \mathrm{~cm}^{-3}, N_{D}=3 \cdot 10^{19} \mathrm{~cm}^{-3}$ ), showing the box approximation is still approximately valid for high doping concentrations and is not significantly affected by electron beam excitation, i.e. low level injection. (b) Electron and hole concentration (electron beam excitation at the intrinsic point) for $n_{i, p}=n_{i, n}$ and reduced doping, also showing the validity of the box approximation and low level injection, along with the expected shift of the intrinsic point away from $x=0$. (c) Same as (b), but for $n_{i, p}=100 \cdot n_{i, n}$. The spikes at $\mathrm{x}=0$ need more elaborated simulations to explain, and to exclude numerical problems.

Full STEBIC profiles could not be simulated using this heterojunction model with the described parameters, as the simulations do not converge for other electron beam exci- 
tation position or produce non-believable STEBIC profile shapes. Still, the advantage of the implemented model is that it relies solely on the actually known parameters in PCMO and STNO, and that all parameter jumps at the chemical interface vanish in the case of homojunction parameters. Consequently, it is recommended that this COMSOL heterojunction model should be further investigated to isolate the reason for numerical problems in the future.

It should be noted that all COMSOL models discussed in this work are within the boundaries of a rigid band approximation. A comparison of the space charge region size $d_{\mathrm{PCMO}}=0.2 \mathrm{~nm}$ and $d_{\mathrm{STNO}}=27 \mathrm{~nm}$ to the Debye lengths of $L_{D y, \mathrm{PCMO}}=0.08 \mathrm{~nm}$ and $L_{D y, \mathrm{STNO}}=3.8 \mathrm{~nm}$ yields that in PCMO, the space charge region is not much larger than the Debye length (even though the PCMO space charge region may be extended to $2.5 \mathrm{~nm}$ due to $\mathrm{Mn}$ and Ti atom interdiffusion $[9])$. Consequently, calculating the extension of the PCMO space charge region by the box approximation (Eq. 2.31 and 2.32) is questionable. Furthermore, quantum mechanical effects like Friedel oscillations could be important, which would require a completely different model.

Furthermore, the nature of strongly correlated charge carriers is, so far, only taken into account effectively, e.g. by small charge carrier mobilities. The nature of polarons may change from small to large polarons when crossing the interface from PCMO to STNO, as the polaron charge carrier type is different in both materials. Charge transfer at the interface may also be hindered by possible orbital mismatch, i.e. it is unclear what the exact excitation energy of minority charge carriers from PCMO actually contributing to EBIC is. The relatively small bandwidth of PCMO, compared to e.g. Si, might also hinder charge transfer from PCMO to STNO [15, 82, 150]. Nevertheless, a rigid band model for PCMO-STNO has been used before successfully to confirm results from photovoltaic and EELS measurements [9]. 


\subsection{Summarizing discussion}

In this part, a summary of the discussion from chapters 4, 5, 6, and 7, as well as from the additional discussion in the preceding part of this chapter, is given, discussing the core findings of the whole work. These are

- The PCMO $(x=0.34)$ excess charge carrier diffusion length at room temperature $L_{\mathrm{PCMO}}=3 \mathrm{~nm}$ has been determined for the first time, and a larger one can be estimated at low temperatures. The STNO diffusion length at room temperature is $L_{\mathrm{STNO}}=17 \mathrm{~nm}$, and no change has been found for low temperatures.

- High resolution (nanometer range) cross section STEBIC can be performed in the SEM (using low energy STEBIC) and, with even better resolution, in the TEM (high resolution STEBIC) in systems with small diffusion length.

- The effect of 'dead' layers at lamella surfaces on EBIC signal was clearly demonstrated from wedge shaped cross section lamellas, and thickness dependent surface recombination can be modeled analytically.

- Using the first combination of STEBIC and simultaneous EELS measurements, a shift of the STEBIC profile maximum away from the chemical PCMO-STNO interface, but within the space charge region has been determined and can be attributed to asymmetric p- and n-doping.

- Modeling of STEBIC profiles using the simple convolution of the electron beam generation volume with a given collection function profile as well as finite element modeling already reproduces the rough profile shape.

A summary of the principle discussion of these results is given in the following.

\subsubsection{Influence of the generation volume}

The electron beam generation volume is confirmed to have a profound influence on cross section EBIC profiles, on PCMO-STNO as well as Si-based p-n junctions. It is possible to model this influence by Monte Carlo simulations of the generation volume. However, the best way to directly understand cross section EBIC profiles is to avoid the (at least lateral) generation volume influence altogether by transitioning to a cylindrical source (lateral point source with uniform strength in all depths of the lamella). There are basically two ways to reduce this influence:

The first is decreasing the acceleration voltage, thus reducing the extension of the generation volume. This is called Bethe limit [160], and is explored in chapter 5. The advantage of this approach is that it works for arbitrary lamella thickness, and it may also be employed for EBIC measurements along a sample cross section prepared at the sample edges, skipping the lamella preparation. Still, in order to understand the EBIC profile the sample edges have to be cleaned and evened out in some way, for example by cleaving, FIB or low energy ion milling. The limitation of this approach is that (1) with ever reducing acceleration voltage (it would be possible to go below $1 \mathrm{kV}$ in the NanoSEM and still get good SE image quality), the signal gets very weak and might be drowned in noise, and (2), possibly more severe, with reducing generation volume, the interaction of the electron beam gets limited to the regions near the sample beam entry surface. For 
example, going below $2 \mathrm{kV}$ reduces the mean depth of the generation volume to below $50 \mathrm{~nm}$ in PCMO and STNO. Because of the surface influence, which will be discussed in the following, no considerable amount of excited charge carriers may be collected by the junction in this case.

The second is increasing the acceleration voltage and choosing small sample thickness. This is the Goldstein limit [161], which is explored in chapter 6, bringing the STEBIC approach to high resolution. As shown by Monte Carlo simulations, the cylindrical source condition is best approached in this case, i.e. the generation volume is not limited to the beam entry surface. While beam entry as well as beam exit surface influence the EBIC profile, excitations in the center of the lamella can safely be collected if the diffusion length is small enough. However, this approach is limited to thin lamellas and is best conducted in a TEM, which requires a rather complex preparation and is less flexible in ambient conditions, e.g. special sample holders have to be used in order to change the temperature.
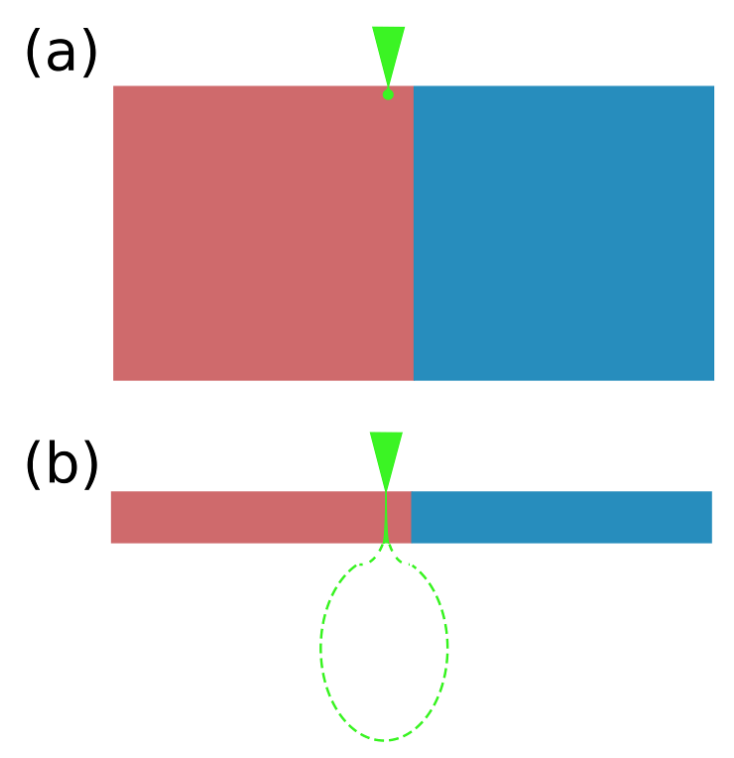

Figure 8.8: Limits of spatial resolution description for an extended electron beam generation volume. (a) Bethe limit: Resolution is determined by small generation volume, (b) Goldstein limit: Resolution is determined by small sample thickness.

\subsubsection{Impact of the sample surface(s)}

The lamella surface has been found to severely limit STEBIC profile measurements, in SEM as well as TEM. Maxima and decay lengths of the STEBIC profiles decrease with decreasing thickness, an effect which could be separated from influences of the generation volume by moving to cylindrical source conditions. However, in these conditions thin lamellas are needed, enhancing the surface influence.

Two aspects of surface influence have been examined: the so-called 'dead' layers and surface recombination. Both are responsible for increased recombination of minority charge carriers, which therefore cannot contribute to the STEBIC signal. It is assumed that any electron-hole pair excited in the dead layers immediately recombines. Therefore, the effective sample thickness can be treated as physical thickness $t$ reduced by dead layer 
thickness $t_{d}$ on the beam entry and the beam exit surface, i.e. $t_{e f f}=t-2 t_{d}$. Accordingly, the effective lamella surface is moved to the interface between electrically active lamella region and dead layer, see Fig. 8.9.

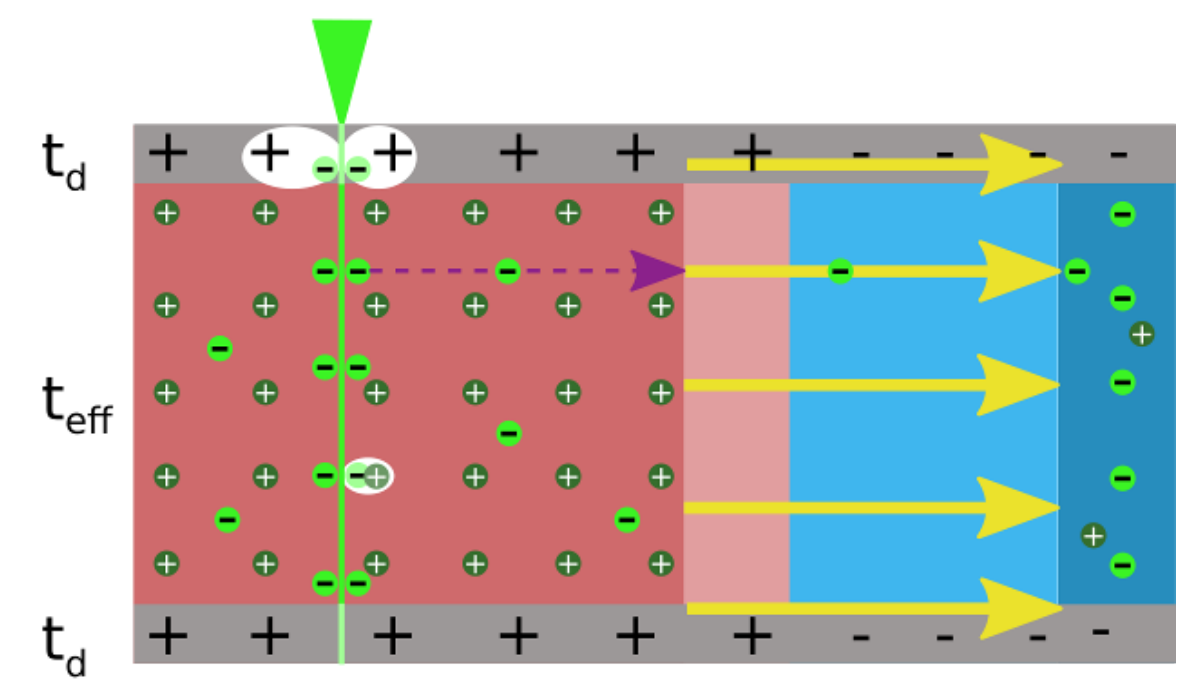

Figure 8.9: Charge carrier processes in a p-n (left: p-doped, right: n-doped) junction with dead layers (thickness $t_{d}$ ) impinged by an electron beam (triangle). The cylindrical shape generation volume (vertical line), recombination (round areas) and diffusion (dashed line) processes of minority charge carriers in the p-doped region (left) are shown. Diffusion to the space charge region (pale area) and collection by the electric field (big arrow lines) leads to electrons from the p-doped region crossing over to the ndoped region, thereby contributing to EBIC. In the dead layers, all generated charge carriers recombine at traps (big + ), preventing their collection.

Recombination at the (effective) lamella surface is a kinetic process limited by the finite surface recombination velocity. A way has been shown to estimate the effect on the STEBIC profile decay length, or effective diffusion length, in the neutral region, by only taking into account the effective lamella thickness and surface recombination velocity. This may be treated more exactly by modeling a two- or three dimensional collection function for the whole neutral lamella region [50].

However, this treatment requires solving the diffusion equation for a charge carrier with a several thousand image charges, a rather tedious procedure. Even if this would be done, the problem remains that this is only valid for the neutral region. Processes in the space charge region are more complicated due to the electric field, and varying injection as discussed before. In both the neutral and the charged region, it is also possible that the surfaces include charged defects, producing electric fields at the surfaces. This may be attributed to Fermi level pinning. In the space charge region, it is expected that the junction and the surface field would even interact in some way, further complicating the situation.

It is expected that this can only truly be understood by finite element simulations which incorporate these effects. 


\subsubsection{Effect of the space charge region}

Even in cases of a small generation volume, STEBIC profiles are not constant within the space charge region, as would be expected from the collection function model with a point source 47]. It was suggested this is due to changing electric field within the SCR [31]. However in PCMO-STNO the strength of the electric field is so high on such a small scale (due to the small space charge region) that this is not believed to be the dominant factor here. Instead, the role of the local injection, which is rarely considered in literature [162], has to be taken into account. Due to band bending, the local injection rises, leading to high injection conditions nearly everywhere in the space charge region (see Fig. 8.10). Assuming constant collection of injected charge carriers in the space charge region, this could explain the STEBIC profile shape in there. Outside the space charge region, the injection is constant (albeit different in both materials if they are doped asymmetrically), and the diffusion process becomes dominant.

The role of the possible electric field at the sample surfaces, and whether it even exists, is still unknown for the system investigated here. The interaction of such a surface field with the electric field of the space charge region may induce a change of band bending. Furthermore, it has been shown in Fig. 8.10 that high injection conditions are prevalent in the space charge region when exciting there with an electron beam, even if low injection is insured in the neutral regions (see also [162]). However, the effect of the larger number of additional charge carriers on the electronic potential has not been considered yet.

Both effects might be important to explain the clearly found lamella thickness dependent shift of the STEBIC profile maximum. For example, the position of the intrinsic point, which is clearly connected to STEBIC profile maximum, might change with the band bending.

Still, the linearity of the EBIC signal maximum with the beam current points to low injection conditions [51] (see Fig. 7.4(a); for TEM-based STEBIC at least until a lamella thickness of $120 \mathrm{~nm}$, above high injection conditions may set in, see Fig. 6.4(a)). A more systematic study of this apparent contradiction is necessary for future work.

\subsubsection{Determining the diffusion length}

One of the main goals of this work was to determine the excess charge carrier diffusion length of PCMO and STNO. In the current state of modeling, the best conditions for achieving this are explained in the following: It is advisable to produce a TEM lamella to perform STEBIC profiles at high acceleration voltages, e.g. $80 \mathrm{kV} .300 \mathrm{kV}$ has proven to produce similar results, as the generation source size is not significantly different, at least up to a thickness of $200 \mathrm{~nm}$. The overall signal is still high enough to be recorded with the used setup. The sample thickness should not be chosen too small, as surface recombination and dead layers influence the STEBIC profiles in that case. For a PCMOSTNO junction, a thickness of around $200 \mathrm{~nm}$ has proven a good compromise between the effects of surface and generation volume.

For other material systems, the expected diffusion length should be used as a guide in thickness choice. If it is larger, the necessary sample thickness also becomes larger, as too many charge carriers would diffuse to the surfaces otherwise. The ideal thickness should be at least, taking diffusion length $L$ and dead layer thickness $t_{d}$ into account, $t_{i d}=2 \cdot\left(L+t_{d}\right)$. When moving to larger sample thickness than $200 \mathrm{~nm}$, it is advisable to use $300 \mathrm{kV}$ acceleration voltage to minimize the generation volume. If the diffusion length 


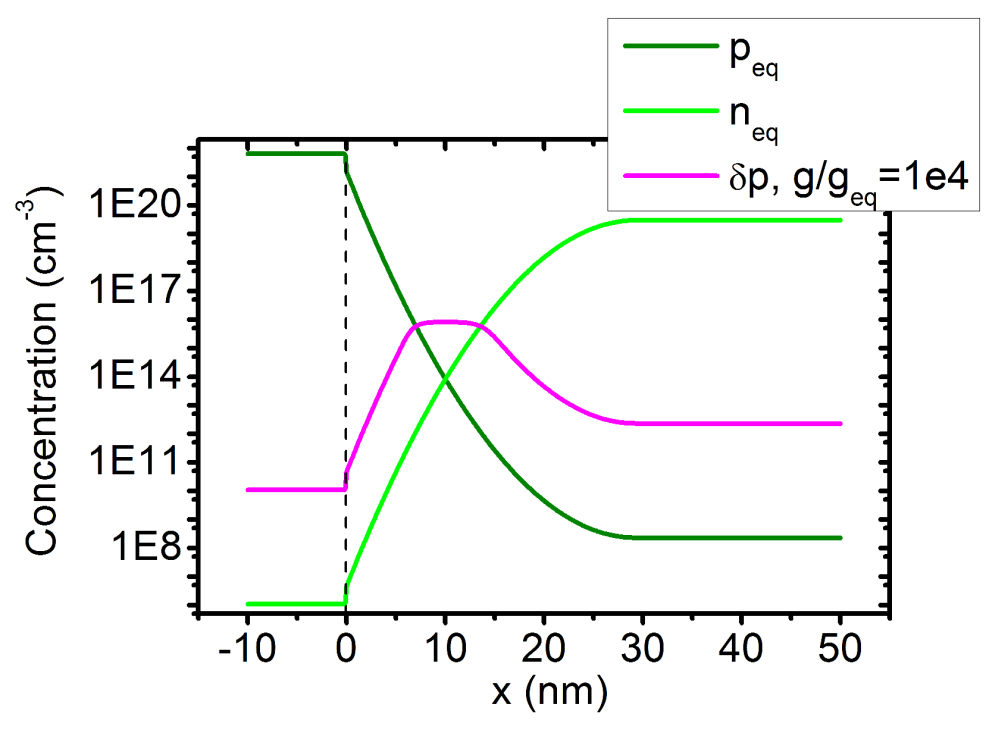

Figure 8.10: Local charge carrier concentrations $p_{e q}$ and $n_{e q}$ in the space charge region in equilibrium, as well as injected charge carrier concentration $\delta p=\delta n$ for a generation rate $g$ far above the thermal generation rate $g_{e q}$, as calculated from Eq. 2.17. Near the intrinsic point $\left(p_{e q}=n_{e q}\right)$, high injection conditions are present, and the injection profile in the space charge region could explain the STEBIC profile shape.

is completely unknown, it is advisable to analyze a wedged shape lamella to extract the STEBIC profile decay length thickness dependently, and check for a plateau of the decay length, where surface and generation volume effects are both not significantly present and thus the decay length would be the real diffusion length.

The found diffusion length of STNO $(17 \mathrm{~nm})$ may be underestimated as the maximum lamella thickness was comparable to the literature diffusion length (around $160 \mathrm{~nm}$, calculated from mobility $\mu_{\mathrm{STNO}}=1 \frac{\mathrm{cm}^{2}}{\mathrm{Vs}_{\mathrm{s}}}[12]$ and lifetime $\tau_{\mathrm{STNO}}=10 \mathrm{~ns}[136 \mid)$, limiting the applicability of our model for the effective diffusion length. Still, the literature value is questionable, as the mobility may differ by orders of magnitude dependent on the exact doping procedure and is not specified by the manufacturer. Furthermore, the lifetime was only estimated $[136]$ for the doping concentration $3 \cdot 10^{19} \mathrm{~cm}^{-3}$ and may actually be smaller.

The literature diffusion length of PCMO $(7 \mathrm{~nm})$ is more clear due to the carefully measured resistivity of prepared PCMO films determining a lower limit of the mobility $\mu_{\mathrm{PCMO}}=0.02 \frac{\mathrm{cm}^{2}}{\mathrm{~V}_{\mathrm{s}}}|76|$ and the lifetime $\tau_{\mathrm{PCMO}}=1 \mathrm{~ns}$ in the low temperature charge ordered phase $[13$. As the lifetime was found to be much smaller at room temperature, the slightly smaller diffusion length measured here $(3 \mathrm{~nm})$ is not surprising. However, macroscopic current-voltage characteristics of optically excited PCMO-STNO junctions suggest that at room temperature, only generated charge carriers at the p-n junction interface contribute to the short circuit current, while in the low temperature charge ordered phase, bulk excitations contribute as well, suggesting a large diffusion length compared to the PCMO space charge region, which has a size below $1 \mathrm{~nm}$ [14. Taking into account the microscopic measurements from this work, the conclusion for room temperature has to be questioned, as a diffusion length of greater size than the space charge region has been measured. 


\subsubsection{Simultaneous EBIC and EELS measurements}

As a new technique in this work, EBIC profiles and EELS spectrum images have been recorded simultaneously. In general, this enables variable evaluation of energy losses in correlation with EBIC signal. It has been used here to determine the chemical interface of PCMO-STNO by mapping the Mn-M edge. Compared to using STEM images for this task, this method is not dependent on local diffraction contrast (for example due to preparation damage and to changing crystal structure at the interface) and thus provides a better estimation of the interface position. Furthermore, the local lamella thickness map for each EBIC profile could be extracted from the EELS maps, providing accurate information about EBIC dependence on local thickness, which is invaluable to determine the thickness dependence of the STEBIC maximum shift (Fig. 6.4(b)).

\subsubsection{Temperature influence}

A strong hint of increased PCMO diffusion length for temperatures below room temperature has been found and attributed to the charge ordered low temperature phase, where an increased lifetime of charge carriers excited through a Jahn Teller transition compared to room temperature was found and explained as metastable state [13. However, the transition temperature could not clearly be determined, as the experiment was conducted with the expected transition around $T=240 \mathrm{~K}$. It could very well be found at temperatures between 250 and $300 \mathrm{~K}$, as the phase transition is rather broad 86,148 150|. Instead of the preliminary analysis using the decay integral, it would be advisable to increase spatial resolution in order to be able to extract the diffusion length from the STEBIC profile decay length. This would require conducting a cooling experiment in the TEM, which needs a special contacted cooling sample holder.

Note that inaccurate temperature measurement cannot be definitely excluded, as the temperature sensor on the SEM cooling stage is not directly located at the sample position. Still, after changing the set temperature, the system is given around 10 minutes to relax into equilibrium. A reliable sign that this actually happened is low drift velocity in the SEM and EBIC images. Comparable experiments and calculations with the same setup and samples have shown a strong temperature variance from sensor to sample to be unlikely 117.

The increasing EBIC signal at low temperatures is not supported by macroscopic measurements of the short-circuit current under optical excitation [12, 14]. A similar behaviour as in Fig. 7.6 was measured for plan view EBIC experiments at the same sample 117]. The apparent contradiction of EBIC and short circuit current measurements remains to be explained. In general, mobility of large polaron charge carriers, which are taking part in the charge transfer process in STNO [72, 75], increases with decreasing temperature [68], which could support charge transport and thus lead to higher EBIC signal. 


\subsection{Outlook}

In the following section, ideas for further work building on the results of this work are suggested.

\subsubsection{Experimental apparatus}

The setup for TEM based EBIC measurements described in this work has already been improved by changing the EBIC current measurement from the direct recording by the electrometer to amplification with the SR570 preamplifier and subsequent recording with a Keithley voltmeter [157] and, later, connecting the voltage output of the amplifier to a STEM detector in the microscope. The latter enables direct synchronization of STEM and EBIC signal by the microscope, faster measuring times as well as noise reduction. Thus, the recording of STEBIC maps with atomic resolution has become possible. Applying a bias voltage in this setup by a voltage source has also already been successfully tested, which can serve as a means to move STEBIC to a different current than the short circuit current to track the spatially resolved current-voltage characteristics. Low ion beam energy cleaning of the surface of TEM lamellas placed on DENS chips is also possible due to an adapter to put DENS chips into a Gatan precision ion polishing system (PIPS).

Due to the charge ordered phase in PCMO, which forms below 200-300 K and features a larger minority charge carrier diffusion length, it would be desirable to study this effect more extensively with atomic resolution in the TEM. This, however, requires a system with stability close to the DENS Lightning system, but with the ability to in situ cool the sample holder, at least with liquid nitrogen.

Another option to increase the versatility of future studies would be to put TEM lamellas on a DENS chip into an SEM, in order to directly compare data taken with TEM typical high acceleration voltages $(80-300 \mathrm{kV})$ to SEM typical low voltages $(<30 \mathrm{kV})$. This would be a considerable advantage from using different samples in different microscopes, as lamella preparation is never fully reproducible, in particular the thickness gradient of a wedge shaped lamella might differ from lamella to lamella. Directly comparing STEBIC profiles from a broad range of acceleration voltages enables a systematic analysis and deeper understanding of the effect of generation volume on the profiles, which may lead the way to a better model.

Conducting off-axis holography on TEM lamellas is a powerful way to determine the actual electrostatic potential development at p-n junctions. The first steps of analysing this for PCMO-STNO shown in this work may be extended to clearly extract the potential by pushing the measurements to a reconstruction with higher spatial resolution, as well as more clearly defined lamella preparation. However, recording STEBIC profiles and holography data on the same lamellas is probably not feasible, as the requirements for the state of the beam entry and beam exit surfaces are complimentary: While they should be as clean as possible for STEBIC, a parallel Ohmic conductance path to the diode at the p-n junction interface is needed for holography to measure the internal potential of the lamella without the charges of impinging electrons. Still, it is certainly interesting to compare the potential of lamellas with different surfaces, i.e. measure the influence of electron beam induced charges on the internal potential of the lamella.

The internal potential and its possible change with charge carrier injection and sample thickness may also be examined from STEBIC profiles by analyzing the profile develop- 
ment within the space charge region. If it can be proven that it is directly connected to local injection, the profile shape there would provide access to band bending by calculating the local charge carrier concentration from the injection level if the generation rate is known. The extension of the space charge region may also be estimated from STEBIC profiles, as it is connected to the profiles' inflection points [141]. In TEM-based STEBIC measurements, they approach the nominal space charge region extension calculated from the box approximation ( $29 \mathrm{~nm}$ for STNO, compare to Fig. 6.4). The exact connection of inflection points and edge of space charge region may be better understood if the role of injection can be substantiated.

Electron energy loss spectra provide access to a large variety of inelastic scattering mechanisms in the examined sample. Mapping elemental edges, or even high energy resolution ELNES spectra, across a p-n junction provides access to the charge transfer mechanism. Combining this directly with EBIC and holography studies can help to determine the prevalent absorption mechanisms in excitation of minority charge carriers and their transfer across the p-n interface on the atomic level. It would be possible to discriminate charge transfer and Jahn Teller excitations in PCMO and the mechanism behind the estimated energy per electron hole pair $E_{\text {eh }}$ could be better understood.

As a more detailed proposal, low loss EELS maps could be used to determine the excitation energy of electron hole pairs $E_{e h}$, as described in Sec. 2.1.4. A way to estimate $E_{e h}$ from STEBIC profiles using a simulated profile from the collection function model has already been shown in Sec. 8.4. As an additional approach, the average energy loss of EELS spectra could be used. It is sufficient to average the energy loss signal in the low loss region, as the most EELS intensity (after the zero loss peak) can usually be found there. However, it is necessary to know the total number of electrons recorded by the EELS camera in order to provide a clear average. This is not trivial, as the EELS entrance aperture in an image filter setup filters out electrons scattered with too large angles. To prevent this, a small camera length should be chosen, as well as elastic scattering conditions be avoided by tilting the sample out of any zone axis. If electron loss can be sufficiently prevented, the local plasmon energy may be extracted from the low loss EELS average energy loss [96], which is the prevalent energy loss mechanism and may be split into single electron hole pair excitation events [163].

\subsubsection{Modeling}

The collection function model has been, up to now, only used with fixed bulk diffusion lengths. Using the analytic model of the thickness dependent decay length, or effective diffusion length, could improve the collection function model. Still, the change of the STEBIC profile shape with varying electron beam acceleration voltage, despite the simulated generation volume being taken into account, is not clearly understood and may show the limit of this model.

Alternatively, numerical solutions of a set of equations including the Poisson equation and continuity equations for electrons and holes may be implemented in COMSOL in order to model the charge collection behaviour of a sample. If the proposed model for a heterojunction can be modified to work reliably, STEBIC linescans can be simulated and compared to experimental results. In an iterative procedure, on both sides the only roughly known intrinsic charge carrier concentrations $n_{i, p}$ and $n_{i, n}$, as well as recombination coefficients $B_{p}$ and $B_{n}$ have to be adjusted to fit the simulated to experimental profiles. It may be necessary to include a dependence of the coefficients $B$ on $p$ and $n$ in 
order to account for a prevalent recombination mechanism other than radiative recombination.

Furthermore, it may be necessary to implement different surface recombination equations in order to account for possible charged surface defects. Additionally, the size of the transition region of several material constants from p- to n-type material could be modeled after electron energy loss data. In the end, from the best fit recombination coefficients, bulk minority charge carrier diffusion lengths can be extracted from the simulation results.

A possibility to enhance the finite element simulations would be the semiconductor nanodevice tool nextnano [164], which solves the Schrödinger equation together with Poisson and continuity equations in order to account for quantummechanical effects on the local density of states due to small device scale.

\subsubsection{Sample system}

Building on the framework for STEBIC experiments and simulation applied in this work to PCMO-STNO junctions with a fixed doping level (Ca doping $x=0.34$ and $\mathrm{Nb}$ doping $y=0.002$ ) can be applied to samples with different doping. A direct doping influence would be the size of the space charge region, which could be confirmed by holography studies of the internal electrostatic potential and also be found in STEBIC profiles. Apart from that, Fig. 2.12 shows the formation of different phases with different electric or magnetic behaviour, which could considerably alter STEBIC profiles, as already demonstrated for the low temperature charge ordered phase. Another interesting comparable sample would be a p-n junction consisting entirely out of PCMO, with different doping levels.

STEBIC and holography could provide access to the band bending in the space charge region, and the charge transfer behaviour across the interface, which is considered to be fairly different from PCMO-STNO, could be potentially tracked by STEBIC as well as EELS. 


\section{Chapter 9}

\section{Summary}

In this work, the goal was to disentangle effects of electron beam generation volume, space charge region, excess charge carrier diffusion length, and sample surfaces which all occur on a nanometer length scale in EBIC experiments on highly doped PCMO-STNO p-n junctions.

By recording thickness dependent low energy STEBIC profiles from cross section wedge shape lamellas and comparing them to Monte Carlo simulated electron beam generation volume, the impact of the generation volume was confirmed and separated from the influence of the lamella surfaces. The latter show up in two forms: The ion beam preparation method induced 'dead' layers in the surface vicinity, which do not contribute to EBIC signal at all, and surface recombination, acting as another excess charge carrier recombination channel alongside bulk recombination, which is controlled by the diffusion length. By comparing PCMO-STNO to Si-based p-n junction lamellas, it was verified that $\mathrm{Si}$ exhibits thicker dead layers than PCMO-STNO, which is reasonable as $\mathrm{Si}$ is much more subject to damage by ion beam irradiation than PCMO or STNO. The dead layer and surface recombination effect was also verified for high energy STEBIC.

While the low energy STEBIC approach makes it possible to measure with a resolution better than $50 \mathrm{~nm}$, the resolution could be substantially increased to the range of $1 \mathrm{~nm}$ in TEM-based high energy STEBIC, as the large acceleration voltage of $80-300 \mathrm{kV}$, combined with a lamella thickness of less than $200 \mathrm{~nm}$ provides conditions of a cylindrical source, i.e. point-like lateral resolution. Simultaneously recording electron energy loss spectra, the chemical p-n junction interface as well as the local lamella thickness was measured.

By using an analytical model to estimate effects of surface recombination on both lamella surfaces, in these experimental conditions the diffusion length of PCMO and STNO could be determined to be $L_{\mathrm{PCMO}}=3 \mathrm{~nm}$ and $L_{\mathrm{STNO}}=17 \mathrm{~nm}$, respectively, which are the first directly measured values for a p-n junction of this material system. This is within the range suggested by measurements of excess charge carrier lifetime, which can be converted to diffusion lengths using the more or less known carrier mobilities. The determined PCMO diffusion length suggests that charge carriers generated at least some nanometers away from the space charge region contribute to the room temperature EBIC signal, which is contradictory to findings from macroscopic optical excitation experiments suggesting that only generation at the p-n junction interface contributes to measured room temperature short circuit current [14]. Future work will require reconciling the nanometer size diffusion length with the macroscopic electrical characteristics.

By cooling a sample and using low energy STEBIC, an increase of the PCMO diffusion length was estimated, as expected from increased lifetime measured in the low temperature charge ordered phase [13]. Notably, the simultaneous recording of STEBIC and 
electron energy loss spectra is first performed here and enables future studies of the electron hole pair excitation process and charge carrier transfer across the $\mathrm{p}-\mathrm{n}$ junction interface.

As a benefit of the high spatial resolution, a shift of the STEBIC profile maximum away from the chemical p-n junction interface into the STNO space charge region was unambiguously determined, which can only be explained by the simultaneous shift of the intrinsic point in an asymmetrically doped p-n junction. This suggests the importance of the local injection level within the space charge region, which is a rarely explored concept for any p-n junction [162]. The high spatial resolution STEBIC setup developed in this work provides the basis of understanding the local injection level effect in the space charge region, but also requires sophisticated modeling of STEBIC data.

Furthermore, the PCMO-STNO space charge region was measured by off-axis electron holography, by reconstructing an object wave phase map and thereby gaining a map of the inner potential of a lamella. Taking into the account the limited spatial resolution of these first experiments, results are in agreement with the space charge region size calculated from the doping levels using the standard box approximation. This method may be clearly improved by focusing future experiments on obtaining object wave reconstructions with a better resolution. The approach may be assisted by taking into account the STEBIC profile inflection points as another measure for the extension of the space charge region 141.

At last, first steps have been taken to model STEBIC profiles. In the first model, a Monte Carlo simulated electron beam generation volume was convoluted with a simple collection function model, which incorporates the size of the space charge region and the diffusion length, but neglects sample surfaces. The model succeeds in reproducing the rough shape of STEBIC profiles, but fails to follow the experimental profiles for sets of profiles measured with different electron beam acceleration voltages. Even though it may be extended in several spatial dimensions or by using lamella thickness dependent effective diffusion lengths, this model may only serve as a guide to quantitatively simulate STEBIC profiles.

In the second model, finite element simulations have been carried out to solve the Poisson equation as well as charge carrier continuity equations simultaneously over a simulated lamella, and simulating STEBIC profiles by moving an external electron hole pair source across the lamella. The general effects of varying doping and diffusion length are already visible, however a robust heterojunction model has to be developed for future simulations. A consistent and versatile model was suggested, but needs more in-depth testing in order to build a robust "STEBIC simulator". It should be noted that such a simulator is not needed to extract the diffusion length from TEM-based high energy STEBIC profiles, as analytical modeling is sufficient to take into account the surface role. Still, detailed modeling is crucial to understand effects within the space charge region.

The framework of experimental methods and simulation models used in this work can readily be applied to p-n or Schottky junctions constructed from other material systems with a diffusion length in the range below $1 \mu \mathrm{m}$, such as organic perovskites. First and foremost, the application to PCMO-STNO junctions with other dopings than used in this work is eligible, opening the way to explore other PCMO phases in its rich phase diagram and better the understanding of charge transfer processes in the promising material. 


\section{Bibliography}

[1] W. Shockley and H. J. Queisser. "Detailed balance limit of efficiency of pn junction solar cells". In: Journal of applied physics 32.3 (1961), p. 510519.

[2] M Yamaguchi. "Physics and technologies of superhigh-efficiency tandem solar cells". In: Semiconductors 33.9 (1999), pp. 961-964. DOI: 10.1134/1.1187812.

[3] M A Green. "Third generation photovoltaics: Ultra-high conversion efficiency at low cost". In: Progress in Photovoltaics: Research and Applications 9.2 (2001), pp. 123-135. DOI: 10.1002/pip.360.

[4] T Ameri, G Dennler, C Lungenschmied, and C J Brabec. "Organic tandem solar cells: A review". In: Energy \& Environmental Science 2.4 (2009), p. 347. DOI: $10.1039 / \mathrm{b} 817952 \mathrm{~b}$.

[5] E Dagotto. Nanoscale Phase Separation and Colossal Magnetoresistance. Springer Science \& Business Media, Berlin, 2013.

[6] T Kramer, M Scherff, D Mierwaldt, J Hoffmann, and C Jooss. "Role of oxygen vacancies for resistive switching in noble metal sandwiched $\operatorname{Pr}_{0.67} \mathrm{Ca}_{0.33} \mathrm{MnO}_{3-\delta}$ ". In: Appl. Phys. Lett. 110.24 (2017), p. 243502. DOI: 10.1063/1.4985645.

[7] D Mierwaldt, V Roddatis, M Risch, J Scholz, J Geppert, M E Abrishami, and C Jooss. "Environmental TEM Investigation of Electrochemical Stability of Perovskite and Ruddlesden-Popper Type Manganite Oxygen Evolution Catalysts". In: Advanced Sustainable Systems 1.12 (2017), p. 1700109. DOI: 10.1002/adsu. 201700109 .

[8] P Jia, Y Wang, Z Yan, J Gong, L Lin, F Gao, and J-M Liu. "Electronic phase engineering induced thermoelectric enhancement in manganites". In: J. Appl. Phys. 124.3 (2018), p. 034501. DOI: $10.1063 / 1.5026868$.

[9] G Saucke, J Norpoth, C Jooss, D Su, and Y Zhu. "Polaron absorption for photovoltaic energy conversion in a manganite-titanate pn heterojunction". In: Phys. Rev. B 85 (2012), p. 165315. DOI: 10.1103/PhysRevB.85.165315.

[10] M v Zimmermann, C S Nelson, J P Hill, D Gibbs, M Blume, D Casa, B Keimer, Y Murakami, C-C Kao, C Venkataraman, T Gog, Y Tomioka, and Y Tokura. "X-ray resonant scattering studies of orbital and charge ordering in $\mathrm{Pr}_{1-x} \mathrm{Ca}_{x} \mathrm{MnO}_{3}$ ". In: Phys. Rev. B 64.19 (2001). DOI: 10.1103/physrevb.64.195133.

[11] A Sawa, T Fujii, M Kawasaki, and Y Tokura. "Highly rectifying $\operatorname{Pr}_{0.7} \mathrm{Ca}_{0.3} \mathrm{MnO}_{3}$ / $\mathrm{SrTi}_{0.9998} \mathrm{Nb}_{0.0002} \mathrm{O}_{3}$ p-n junction". In: Appl. Phys. Lett. 86 (2005), p. 112508. DOI: $10.1063 / 1.1883336$.

[12] B Ifland, P Peretzki, B Kressdorf, P Saring, A Kelling, M Seibt, and C Jooss. "Current-voltage characteristics of manganite-titanite perovskite junctions". In: Beilstein J. Nanotechnol. 6 (2015), pp. 1467-1484. DOI: 10.3762/bjnano.6.152. 
[13] D Raiser, S Mildner, B Ifland, M Sotoudeh, P E Blöchl, S Techert, and C Jooss. "Evolution of Hot Polaron States with a Nanosecond Lifetime in a Manganite Perovskite". In: Advanced Energy Materials 7.12 (2017), p. 1602174. ISSN: 16146840. DOI: $10.1002 /$ aenm. 201602174.

[14] B Ifland, J Hoffmann, B Kressdorf, V Roddatis, M Seibt, and C Jooss. "Contribution of Jahn-Teller and charge transfer excitations to the photovoltaic effect of manganite/titanite heterojunctions". In: New Journal of Physics 19.6 (2017), p. 063046 .

[15] S Mildner, J Hoffmann, P E Blöchl, S Techert, and C Jooss. "Temperature and doping dependent optical absorption in the smallpolaron system $\operatorname{Pr}_{1-x} \mathrm{Ca}_{x} \mathrm{MnO}_{3}$ ". In: Physical Review B 92.3 (2015), p. 035145.

[16] S M Sze and Kwok K Ng. Physics of Semiconductor Devices. 3rd ed. Hoboken, New Jersey: John Wiley and Sons, 2006.

[17] H J Leamy. "Charge collection scanning electron microscopy". In: J. Appl. Phys. 53 (1982), R51. DOI: 10.1063/1.331667.

[18] E B Yakimov. "What is the real value of diffusion length in GaN?" In: Journal of Alloys and Compounds 627 (2015), pp. 344-351. DOI: 10.1016/j.jallcom. 2014. 11.229.

[19] C Donolato. "An analytical model of SEM and STEM charge collection images of dislocations in thin semiconductor layers, I. Minority Carrier Generation, Diffusion, and Collection". In: Phys. Stat. Sol. (a) 65 (1981), p. 649. DOI: 10.1002/ pssa.2210650231.

[20] C J Wu and D B Wittry. "Investigation of minority-carrier diffusion lengths by electron bombardment of Schottky barriers". In: J. Appl. Phys. 49.5 (1978), pp. 2827-2836. DOI: $10.1063 / 1.325163$

[21] W Van Roosbroeck. "Injected Current Carrier Transport in a Semi-Infinite Semiconductor and the Determination of Lifetimes and Surface Recombination Velocities". In: J. Appl. Phys. 26.4 (1955), pp. 380-391. DOI: 10.1063/1.1722002.

[22] K L Luke, O von Roos, and Li-jen Cheng. "Quantification of the effects of generation volume, surface recombination velocity, and diffusion length on the electronbeam-induced current and its derivative: Determination of diffusion lengths in the low micron and submicron ranges". In: J. Appl. Phys. 57.6 (1985), pp. 1978-1984. DOI: $10.1063 / 1.334382$

[23] P Peretzki, B Ifland, C Jooss, and M Seibt. "Low energy scanning transmission electron beam induced current for nanoscale characterization of p-n junctions". In: Phys. Status Solidi RRL (2017), p. 1600358. DOI: 10.1002/pssr.201600358.

[24] J P McKelvey. Solid State and Semiconductor Physics. New York: Harper and Row Publishers, 1966.

[25] C A Klein. "Bandgap Dependence and Related Features of Radiation Ionization Energies in Semiconductors". In: J. Appl. Phys. 39 (1968), p. 2029. DoI: 10.1063/ 1.1656484 .

[26] V I Fistul. Heavily Doped Semiconductors. Plenum Press, New York, 1995.

[27] P Würfel. Physics of Solar Cells. Wiley-VCH, Weinheim, 2005. 
[28] J L Gray. "The Physics of the Solar Cell". In: Handbook of Photovoltaic Science and Engineering. Ed. by A Luque and S Hegedus. Chichester, West Sussex, England: John Wiley and Sons, 2003.

[29] H Fuhrmann, A Candel, M Döbeli, and R Mühle. "Minimizing damage during focused-ion-beam induced desorption of hydrogen". In: Journal of Vacuum Science ES Technology B 17 (1999), p. 2443. DOI: 10.1116/1.591108.

[30] B R Weinberger, H W Deckman, E Yablonovitch, T Gmitter, W Kobasz, and S Garoff. "The passivation of electrically active sites on the surface of crystalline silicon by fluorination". In: J. Vac. Sci. Technol. A 3.3 (1985), pp. 887-891. DOI: $10.1116 / 1.573340$.

[31] P M Haney, H P Yoon, B Gaury, and N B Zhitenev. "Depletion region surface effects in electron beam induced current measurements". In: J. Appl. Phys. 120 (2016), p. 095702. DOI: 10.1063/1.4962016.

[32] A G Milnes and D L Feucht. Heterojunctions and Metal-Semiconductor Junctions. New York and London: Academic Press, 1972.

[33] W R Smith. "P-N junction capacitance". In: Int. J. Electronics 31.3 (1971), pp. 201215. DOI: $10.1080 / 00207217108938217$.

[34] W Shockley. "The Theory of p-n Junctions in Semiconductors and p-n Junction Transistors". In: Bell System Technical Journal 28.3 (1949), pp. 435-489. DOI: 10.1002/j.1538-7305.1949.tb03645.x.

[35] K R McIntosh, P P Altermatt, and G Heiser. "Depletion-region recombination in silicon solar cells: When does $m_{D R}=2$ ?" In: Proceedings of the 16th European Photovoltaic Solar Energy Conference. 2000, pp. 251-254.

[36] V Nadenau, U Rau, A Jasenek, and H W Schock. "Electronic properties of CuGaSe ${ }_{2}$ based heterojunction solar cells. Part I: Transport analysis". In: J. Appl. Phys. 87 (2000), p. 584. DOI: $10.1063 / 1.371903$

[37] C Leach. "The Effect of Voltage Bias on the EBIC Contrast Present at Varistor Grain Boundaries". In: Interface Science 8.4 (2000), pp. 375-387. DOI: 10.1023/a: 1008783813055

[38] M Kittler and W Seifert. "EBIC defect characterisation: state of understanding and problems of interpretation". In: Materials Science and Engineering: B 42.1-3 (1996), pp. 8-13. DOI: 10.1016/s0921-5107(96)01677-7.

[39] V Kveder, M Kittler, and W Schröter. "Recombination activity of contaminated dislocations in silicon: A model describing electron-beam-induced current contrast behavior". In: Phys. Rev. B 63.11 (2001), p. 115208. DOI: 10.1103/physrevb.63. 115208 .

[40] V I Orlov, O V Feklisova, and E B Yakimov. "A Comparison of EBIC, LBIC and XBIC Methods as Tools for Multicrystalline Si Characterization". In: Solid State Phenomena 205-206 (2013), pp. 142-147. DOI: 10.4028/www.scientific.net/ ssp. 205-206.142

[41] C Donolato. "On the analysis of diffusion length measurements by SEM". In: SolidState Electronics 25.11 (1982), pp. 1077-1081. DOI: 10.1016/0038-1101(82) 90144-7. 
[42] P H Rekemeyer, C-H M Chuang, M G Bawendi, and S Gradecak. "Minority Carrier Transport in Lead Sulfide Quantum Dot Photovoltaics". In: Nano Letters 17 (2017), pp. 6221-6227. DOI: 10.1021/acs.nanolett.7b02916.

[43] J W Orton and P Blood. The Electrical Characterization of Semiconductors: Measurement of Minority Carrier Properties. Ed. by N H March. Techniques of Physics. London: Academic Press, 1990, p. 111.

[44] A Rothwarf. "CuInSe $2 / \mathrm{Cd}(\mathrm{Zn}) \mathrm{S}$ solar cell modeling and analysis". In: Solar Cells 16 (1986), pp. 567-590. DOI: 10.1016/0379-6787(86)90110-9.

[45] C Donolato. "An alternative proof of the generalized reciprocity theorem for charge collection". In: J. Appl. Phys. 66.9 (1989), pp. 4524-4525. DOI: 10.1063/1.343932.

[46] M v Laue. "Die Absorption der Röntgenstrahlen in Kristallen im Interferenzfall". In: Acta Crystallographica 2.2 (1949), pp. 106-113. DOI: 10.1107/s0365110x49000242.

[47] M Nichterwitz and T Unold. "Numerical simulation of cross section electron-beam induced current in thin-film solar-cells for low and high injection conditions". In: J. Appl. Phys. 114 (2013), p. 134504. DOI: 10.1063/1.4823519.

[48] W H Hackett. "Electron-Beam Excited Minority-Carrier Diffusion Profiles in Semiconductors". In: J. Appl. Phys. 43.4 (1972), pp. 1649-1654. DOI: 10.1063/1. 1661375 .

[49] L Jastrzebski, J Lagowski, and H C Gatos. "Application of scanning electron microscopy to determination of surface recombination velocity: GaAs". In: Appl. Phys. Lett. 27.10 (1975), pp. 537-539. DOI: 10.1063/1.88276

[50] C C Tan, V K S Ong, and K Radhakrishnan. "The study of the charge collection of the normal-collector configuration". In: Prog. Photovolt: Res. Appl. 21.5 (2012). DOI: $10.1002 /$ pip. 2190

[51] J Y Chi and H C Gatos. "Determination of dopant-concentration diffusion length and lifetime variations in silicon by scanning electron microscopy". In: Journal of Applied Physics 50.5 (1979), pp. 3433-3440. DOI: 10.1063/1.326336.

[52] P M Haney, H P Yoon, P Koirala, R W Collins, and N B Zhitenev. "Electron beam induced current in photovoltaics in the high injection regime". In: Nanotechnology 26 (2015), p. 295401. DOI: 10.1088/0957-4484/26/29/295401.

[53] R L Anderson. "Experiments on Ge-GaAs Heterojunctions". In: Solid-State Electronics 5 (1962), pp. 341-351. DOI: 10.1016/0038-1101(62)90115-6.

[54] J E Sutherland and J R Hauser. A theoretical study of heterojunction and graded band gap type solar cells. Tech. rep. North Carolina State Univ.; Dept. of Electrical Engineering.; Raleigh, NC, United States, 1977. URL: https://ntrs.nasa.gov/ search. $j$ sp?R=19770023674 (visited on 11/17/2018).

[55] M A Green, A Ho-Baillie, and H J Snaith. "The emergence of perovskite solar cells". In: Nature Photonics 8.7 (2014), pp. 506-514. DOI: 10.1038/nphoton . 2014.134

[56] V M Goldschmidt. "Die Gesetze der Krystallochemie". In: Die Naturwissenschaften 14.21 (1926), pp. 477-485. DOI: $10.1007 /$ bf 01507527. 
[57] Y Tokura and Y Tomioka. "Colossal magnetoresistive manganites". In: Journal of Magnetism and Magnetic Materials 200 (1999), pp. 1-23. DOI: 10.1016/s03048853(99) 00352-2.

[58] H Y Hwang, S-W Cheong, P G Radaelli, M Marezio, and B Batlogg. "Lattice Effects on the Magnetoresistance in Doped LaMnO3". In: Phys. Rev. Lett. 75.5 (1995), pp. 914-917. DOI: 10.1103/physrevlett.75.914.

[59] L D Landau and S I Pekar. "Effective mass of a polaron". In: J. Exptl. Theoret. Phys. (1948).

[60] I G Austin and N F Mott. "Polarons in crystalline and non-crystalline materials". In: Advances in Physics 18.71 (1969), pp. 41-102. DOI: 10.1080/00018736900101267.

[61] A Braun and Q Chen. "Experimental neutron scattering evidence for proton polaron in hydrated metal oxide proton conductors". In: Nat. Commun. 8 (2017), p. 15830 . DOI: $10.1038 /$ ncomms 15830

[62] T Holstein. "Studies of polaron motion: Part II. The "small" polaron". In: Annals of Physics 8.3 (1959), pp. 343-389. DOI: 10.1016/0003-4916(59)90003-x.

[63] D. Feinberg, S. Ciuchi, and F. de Pasquale. "Squeezing phenomena in interacting electron-phonon systems". In: International Journal of Modern Physics B 4.7\&8 (1990), pp. 1317-1367. DOI: 10.1142/s0217979290000656.

[64] H Böttger and V V Bryksin. Hopping Conduction in Solids. Akademie Verlag, Berlin, 1985.

[65] I G Lang and Yu A Firsov. "Mobility of small-radius polarons at low temperatures". In: J. Exptl. Theoret. Phys. 18.1 (1964), p. 262.

[66] R P Feynman, R W Hellwarth, C K Iddings, and P M Platzman. "Mobility of Slow Electrons in a Polar Crystal". In: Phys. Rev. 127.4 (1962), pp. 1004-1017. DOI: 10.1103 /physrev.127.1004.

[67] P Moschkau. "Defektabhängige Transporteigenschaften von Praseodym-KalziumManganat". PhD thesis. Georg-August-Universität Göttingen, 2009.

[68] D Emin. "Polarons". In: 10th ed. Vol. 14. McGraw-Hill concise encyclopedia of science \& technology. McGraw-Hill, 2007.

[69] O N Tufte and P W Chapman. "Electron Mobility in Semiconducting Strontium Titanate". In: Phys. Rev. 155.3 (1967), pp. 796-802. DOI: 10.1103/physrev.155. 796.

[70] H P R Frederikse and G A Candela. "Magnetic Susceptibility of Insulating and Semiconducting Strontium Titanate". In: Phys. Rev. 147.2 (1966), pp. 583-584. DOI: $10.1103 /$ physrev.147.583.

[71] J L M van Mechelen, D van der Marel, C Grimaldi, A B Kuzmenko, N P Armitage, N Reyren, H Hagemann, and I I Mazin. "Electron-Phonon Interaction and Charge Carrier Mass Enhancement in $\mathrm{SrTiO}_{3}$ ". In: Phys. Rev. Lett. 100.22 (2008), p. 226403. DOI: 10.1103/physrevlett.100.226403.

[72] C Z Bi, J Y Ma, J Yan, X Fang, B R Zhao, D Z Yao, and X G Qiu. "Electronphonon coupling in Nb-doped $\mathrm{SrTiO}_{3}$ single crystal". In: Journal of Physics: Condensed Matter 18.8 (2006), pp. 2553-2561. DOI: 10.1088/0953-8984/18/8/017. 
[73] J A Noland. "Optical Absorption of Single-Crystal Strontium Titanate". In: Phys. Rev. 94.3 (1954), pp. 724-724. DOI: 10.1103/physrev.94.724.

[74] D M Eagles, M Georgiev, and P C Petrova. "Explanation for the temperature dependence of plasma frequencies in $\mathrm{SrTiO}_{3}$ using mixed-polaron theory". In: Phys. Rev. B 54.1 (1996), pp. 22-25. DOI: 10.1103/physrevb.54.22.

[75] X Hao, Z Wang, M Schmid, U Diebold, and C Franchini. "Coexistence of trapped and free excess electrons in $\mathrm{SrTiO}_{3}$ ". In: Phys.Rev. B 91.8 (2015), p. 085204. DOI: 10.1103/physrevb.91.085204.

[76] G Saucke. "Untersuchung eines korrelierten Perowskit-pn-Heteroübergangs". Diplomarbeit. Georg-August-Universität Göttingen, 2011.

[77] B Ifland. "Untersuchung von pn-Heterostrukturen aus Manganat und Titanat". Diploma thesis. Georg-August-Universität Göttingen, 2011.

[78] Z Jirák, S Krupička, Z Šimša, M Dlouhá, and S Vratislav. "Neutron diffraction study of $\operatorname{Pr}_{1-x} \mathrm{Ca}_{x} \mathrm{MnO}_{3}$ perovskites". In: Journal of Magnetism and Magnetic Materials 53.1-2 (1985), pp. 153-166. DOI: 10.1016/0304-8853(85)90144-1.

[79] R Gross and A Marx. Lecturescript Spinelektronik. Walther-Meissner-Institut, Lehrstuhl für Technische Physik (E23). 2005.

[80] H A Jahn and E Teller. "Stability of Polyatomic Molecules in Degenerate Electronic States. I. Orbital Degeneracy". In: Proceedings of the Royal Society A: Mathematical, Physical and Engineering Sciences 161.905 (1937), pp. 220-235. DOI: $10.1098 /$ rspa.1937.0142.

[81] O F Schirmer, M Imlau, C Merschjann, and B Schoke. "Electron small polarons and bipolarons in $\mathrm{LiNbO}_{3}$ ". In: J. Phys.: Condens. Matter 21 (2009), p. 123201. DOI: $10.1088 / 0953-8984 / 21 / 12 / 123201$.

[82] M Sotoudeh, S Rajpurohit, P Blöchl, D Mierwaldt, J Norpoth, V Roddatis, S Mildner, B Kressdorf, B Ifland, and C Jooss. "Electronic structure of $\operatorname{Pr}_{1-x} \mathrm{Ca}_{x} \mathrm{MnO}_{3}$ ". In: Phys. Rev. B 95.23 (2017), p. 235150. DOI: 10.1103/physrevb.95.235150.

[83] P Grossmann, I Rajkovic, R Moré, J Norpoth, S Techert, C Jooss, and K Mann. "Time-resolved near-edge x-ray absorption fine structure spectroscopy on photoinduced phase transitions using a tabletop soft-x-ray spectrometer". In: Rev. Sci. Instrum. 83 (2012), pp. 053110-1. DOI: 10.1063/1.4718936

[84] A Odagawa, H Sato, I H Inoue, H Akoh, M Kawasaki, Y Tokura, T Kanno, and $\mathrm{H}$ Adachi. "Colossal electroresistance of a $\mathrm{Pr}_{0.7} \mathrm{Ca}_{0.3} \mathrm{MnO}_{3}$ thin film at room temperature". In: Phys. Rev.B 70.22 (2004), p. 224403. DOI: 10.1103/physrevb. 70.224403 .

[85] A Asamitsu, Y Tomioka, H Kuwahara, and Y Tokura. "Current switching of resistive states in magnetoresistive manganites". In: Nature 388.6637 (1997), pp. 5052. DOI: $10.1038 / 40363$.

[86] C Jooss, L Wu, T Beetz, R F Klie, M Beleggia, M A Schofield, S Schramm, J Hoffmann, and Y Zhu. "Polaron melting and ordering as key mechanisms for colossal resistance effects in manganites". In: Proceedings of the National Academy of Sciences 104.34 (2007), pp. 13597-13602. DOI: 10.1073/pnas.0702748104. 
[87] S Schramm, J Hoffmann, and C Jooss. "Transport and ordering of polarons in CER manganites PrCaMnO". In: Journal of Physics: Condensed Matter 20.39 (2008), p. 395231. DOI: 10.1088/0953-8984/20/39/395231.

[88] R von Helmolt, J Wecker, B Holzapfel, L Schultz, and K Samwer. "Giant negative magnetoresistance in perovskitelike $\mathrm{La}_{2 / 3} \mathrm{Ba}_{1 / 3} \mathrm{MnO}_{x}$ ferromagnetic films". In: Phys. Rev. Lett. 71.14 (1993), pp. 2331-2333. DOI: 10.1103/physrevlett. 71.2331 .

[89] M Scherff, J Hoffmann, B Meyer, T Danz, and C Jooss. "Interplay of crossplane polaronic transport and resistive switching in $\mathrm{Pt}-\mathrm{Pr}_{0.67} \mathrm{Ca}_{0.33} \mathrm{MnO}_{3}$-Pt heterostructures". In: New Journal of Physics 15.10 (2013), p. 103008. DOI: 10.1088/ $1367-2630 / 15 / 10 / 103008$

[90] E Ruska. "Das Entstehen des Elektronenmikroskops und der Elektronenmikroskopie". In: Physik Journal 43.7 (1987), pp. 271-281. DOI: 10.1002/phbl.19870430729.

[91] D B Williams and C B Carter. Transmission Electron Microscopy. 2nd ed. Springer Science \& Business Media, New York, 2009.

[92] L Reimer. Scanning Electron Microscopy. Springer, 1998.

[93] H Rose. "Outline of a spherically corrected semiaplanatic medium-voltage transmission electron microscope". In: Optik 85 (1990), pp. 19-24.

[94] M Haider, S Uhlemann, E Schwan, H Rose, B Kabius, and K Urban. "Electron microscopy image enhanced". In: Nature 392.6678 (1998), pp. 768-769. DOI: 10 . $1038 / 33823$.

[95] M Stöger-Pollach and P Schattschneider. "The influence of relativistic energy losses on bandgap determination using valence EELS". In: Ultramicroscopy 107.12 (2007), pp. 1178-1185. DOI: 10.1016/j.ultramic.2007.01.015.

[96] R F Egerton. Electron Energy-Loss Spectroscopy in the Electron Microscope. Third Edition. Springer, 2011. DOI: 10.1007/978-1-4419-9583-4.

[97] GIF Quantum Owner's Manual. Gatan, Inc. 2010.

[98] J M Cowley. "Twenty forms of electron holography". In: Ultramicroscopy 41.4 (1992), pp. 335-348. DOI: 10.1016/0304-3991(92)90213-4

[99] G Möllenstedt and H Wahl. "Elektronenholographie und Rekonstruktion mit Laserlicht". In: Die Naturwissenschaften 55.7 (1968), pp. 340-341. DOI: 10 . $1007 /$ bf00600454.

[100] H Wahl. "Bildebenenholografie mit Elektronen". Habilitation thesis. Universität Tübingen, 1975.

[101] D Gabor. "A New Microscopic Principle". In: Nature 161.4098 (1948), pp. 777778. DOI: $10.1038 / 161777 \mathrm{aO}$.

[102] W D Rau, P Schwander, F H Baumann, W Höppner, and A Ourmazd. "TwoDimensional Mapping of the Electrostatic Potential in Transistors by Electron Holography". In: Phys. Rev. Lett. 82.12 (1999), pp. 2614-2617. DOI: $10.1103 /$ PhysRevLett.82.2614. 
[103] J B Park, T Niermann, D Berger, A Knauer, I Koslow, M Weyers, M Kneissl, and M Lehmann. "Impact of electron irradiation on electron holographic potentiometry". In: Applied Physics Letters 105.9 (2014), p. 094102. DOI: 10.1063/1. 4894718.

[104] M Linck, B Freitag, S Kujawa, M Lehmann, and T Niermann. "State of the art in atomic resolution off-axis electron holography". In: Ultramicroscopy 116 (2012), pp. 13-23. DOI: 10.1016/j.ultramic.2012.01.019.

[105] H Lichte. "Electron holography approaching atomic resolution". In: Ultramicroscopy 20.3 (1986), pp. 293-304. DOI: 10.1016/0304-3991(86)90193-2.

[106] P Hovington, D Drouin, and R Gauvin. "CASINO: A new Monte Carlo code in C language for electron beam interaction - Part I: Description of the program". In: Scanning 19.1 (1997), pp. 1-14. DOI: 10.1002/sca.4950190101.

[107] D Drouin, A R Couture, D Joly, X Tastet, V Aimez, and R Gauvin. "CASINO V2.42 - A Fast and Easy-to-use Modeling Tool for Scanning Electron Microscopy and Microanalysis Users". In: Scanning 29 (2007), pp. 92-101. DOI: 10.1002/sca. 20000.

[108] H Demers, N Poirier-Demers, A R Couture, D Joly, M Guilmain, N de Jonge, and D Drouin. "Three-dimensional electron microscopy simulation with the CASINO Monte Carlo software". In: Scanning 33.3 (2011), pp. 135-146. DOI: 10.1002/sca. 20262 .

[109] D Drouin, P Hovington, and R Gauvin. "CASINO: A new Monte Carlo code in C language for electron beam interactions - Part II: Tabulated values of the Mott cross section". In: Scanning 19.1 (1997), pp. 20-28. DOI: $10.1002 / \mathrm{sca}$. 4950190103 .

[110] D C Joy and S Luo. "An Empirical Stopping Power Relationship for Low-Energy Electrons". In: Scanning 11 (1989), pp. 179-180. DOI: 10.1002/sca.4950110404.

[111] P Hovington, D Drouin, R Gauvin, D C Joy, and N Evans. "CASINO: A new Monte Carlo code in C language for electron beam interactions - Part III: Stopping power at low energies". In: Scanning 19.1 (1997), pp. 29-35. DOI: 10.1002/sca. 4950190104 .

[112] H Goering, L Tobiska, and H G Roos. Die Finite-Elemente-Methode für Anfänger. Wiley, 2010.

[113] B Ifland, JHoffmann, T Kramer, M Scherff, S Mildner, and C Jooss. "Strain Driven Phase Decomposition in Ion-Beam Sputtered $\operatorname{Pr}_{1-x} \mathrm{Ca}_{x} \mathrm{MnO}_{3}$ Films". In: Journal of Nanomaterials 2015 (2015), pp. 1-12. DOI: 10.1155/2015/935167.

[114] H Spende. "In-situ und ex-situ elektronenmikroskopische Untersuchungen der Legierung von Aluminium und multikristallinem Silizium und des resultierenden pn-Übergangs". MA thesis. Georg-August-Universität Göttingen, 2017.

[115] Nova 600 NanoLab Product Data. FEI Company. 2003.

[116] Specimen Cooling Stage Modules C1000 and CF302. Gatan, Inc. 2015.

[117] T W G Westphal. "Untersuchung von $\mathrm{Pr}_{1-x} \mathrm{Ca}_{x} \mathrm{MnO}_{3}-\mathrm{SrTi}_{1-y} \mathrm{Nb}_{y} \mathrm{O}_{3}$-Grenzflächen mittels elektronenstrahl-induzierter Spannung (EBIV)". MA thesis. Georg-AugustUniversität Göttingen, 2018. 
[118] Model SR570 Low-Noise Current Preamplifier Manual. Revision 1.7. Stanford Research Systems. 2015.

[119] Lightning D7+ A-D6H8-FS Product Manual. DENSsolutions. 2017.

[120] J Lindner. "Entwicklung von in-situ Transmissionselektronenmikroskopie Methodik mit Zeitkorrelation von Abbildung und photoelektrischen Daten". MA thesis. Georg-August-Universität Göttingen, 2018.

[121] M Kittler and J Lärz. "Evaluation of p-n junction position and channel length in Si devices with resolution of a few nanometers by low-energy EBIC". In: Sol. St. Phenom. 63-64 (1998), p. 77. DOI:10.4028/www.scientific.net/ssp.63-64.77.

[122] T G Sparrow and U Valdrèg. "Application of scanning transmission electron microscopy to semiconductor devices". In: Phil. Mag. 36.6 (1977), pp. 1517-1528. DOI: $10.1080 / 14786437708238532$

[123] C Li, J Poplawsky, Y Wu, A R Lupini, A Mouti, D N Leonard, N Paudel, W Yin, M Al-Jassim, Y Yan, and S J Pennycook. "From atomic structure to photovoltaic properties in CdTe solar cells". In: Ultramicroscopy 134 (2013), pp. 113-125. DOI: 10.1016/j.ultramic.2013.06.010.

[124] C Cabanel, D Brouri, and J Y Laval. "Low temperature semi-quantitative analysis of local electrical field in silicon diode by transmission electron microscopy". In: Eur. Phys. J. Appl. Phys. 34 (2006), pp. 107-116. DOI: 10.1051/epjap:2006042.

[125] L. Lu, T. Zheng, Q. Wu, A. M. Schneider, D. Zhao, and L. Yu. "Recent Advances in Bulk Heterojunction Polymer Solar Cells". In: Chem. Rev. 115 (2015), p. 12666. DOI: doi:10.1021/acs.chemrev.5b00098.

[126] N.-G. Park. "Perovskite solar cells: an emerging photovoltaic technology". In: $M a-$ terials Today 18 (2015), p. 65.

[127] M A Falkenberg, H Schuhmann, M Seibt, and V Radisch. "Localization and preparation of recombination-active extended defects for transmission electron microscopy analysis". In: Rev. Sci. Instrum. 81.6, 063705 (2010), p. 063705. DOI: 10.1063/1.3443573.

[128] F Schindler, J Geilker, W Kwapil, W Warta, and M C Schubert. "Hall mobility in multicrystalline silicon". In: J. Appl. Phys. 110 (2011), p. 043722. DOI: 10.1063/ 1.3622620 .

[129] A C Twitchett, R E Dunin-Borkowski, and P A Midgley. "Quantitative Electron Holography of Biased Semiconductor Devices". In: Phys. Rev. Lett. 88.23 (2002). DOI: $10.1103 /$ PhysRevLett.88.238302.

[130] M A Gribelyuk, M R McCartney, J Li, C S Murphy, P Ronsheim, B Doris, J S McMurray, S Hedge, and D J Smith. "Mapping of Electrostatic Potential in Deep Submicron CMOS Devices by Electron Holography". In: Phys. Rev. Lett. 89.2 (2002), p. 025502. DOI: 10.1103/PhysRevLett.89.025502.

[131] M. A. Green. "Third Generation Photovoltaics:Ultra-high Conversion Efficiency at Low Cost". In: Prog. Photovolt: Res. Appl. 9 (2001), p. 123. DOI: DOI:10.1002/ pip.360.

[132] M. Triplett, Y. Yang, F. Léonard, A. Alec Talin, M. Saif Islam, and D. Yu. "Long Minority Carrier Diffusion Lengths in Bridged Silicon Nanowires". In: Nano Letters 15.1 (2014), pp. 523-529. DOI: $10.1021 / \mathrm{nl} 503870 \mathrm{u}$. 
[133] Jingbi You, Letian Dou, Ken Yoshimura, Takehito Kato, Kenichiro Ohya, Tom Moriarty, Keith Emery, ChunChao Chen, Jing Gao, Gang Li, and Yang Yang. "A polymer tandem solar cell with $10.6 \%$ power conversion efficiency". In: Nature Communications 4 (2013), p. 1446.

[134] Martin Neukom, Simon Züfle, Sandra Jenatsch, and Beat Ruhstaller. "Optoelectronic characterization of thirdgeneration solar cells". In: Science and Technology of Advanced Materials 19.1 (2018), p. 291316. DOI: $10.1080 / 14686996.2018$. 1442091 .

[135] G Zhao, Y S Wang, D J Kang, W Prellier, M Rajeswari, H Keller, T Venkatesan, $\mathrm{C}$ W Chu, and R L Greene. "Evidence for the immobile bipolaron formation in the paramagnetic state of the magnetoresistive manganites". In: Phys. Rev. B 62.18 (2000), R11949-R11952. DOI: 10.1103/physrevb.62.r11949.

[136] H Yasuda and Y Kanemitsu. "Dynamics of nonlinear blue photoluminescence and Auger recombination in $\mathrm{SrTiO}_{3}$ ". In: Phys. Rev. B 77 (2008), p. 193202. DoI: 10.1103/PhysRevB.77.193202.

[137] P. M. Petroff, R. A. Logan, and A. Savage. "Nonradiative Recombination at Dislocations in III-V Compound Semiconductors". In: Physical Review Letters 44.4 (1980), pp. 287-291. DOI: 10.1103/PhysRevLett.44.287.

[138] S.J. Pennycook. "Investigation of the electronic effects of dislocations by STEM". In: Ultramicroscopy 7.1 (1981), pp. 99-104. DOI: 10.1016/0304-3991(81)900279 .

[139] M-G Han, J A Garlow, M S J Marshall, A L Tiano, S S Wong, S-W Cheong, F J Walker, C H Ahn, and Y Zhu. "Electron-beam-induced-current and active secondary-electron voltage-contrast with aberration-corrected electron probes". In: Ultramicroscopy 176 (2017), pp. 80-85. DOI: 10.1016/j.ultramic.2017.03.028.

[140] K L Bunker, J C Gonzalez, D Batchelor, T J Stark, and P E Russell. "Development of a High Lateral Resolution Electron Beam Induced Current Technique for Electrical Characterization of InGaN-Based Quantum Well Light Emitting Diodes". In: Mat. Res. Soc. Symp. Proc. 743 (2002), p. L10.10. DOI: 10.1557/PROC-743$\mathrm{L} 10.10$.

[141] G Oelgart, J Fiddicke, and R Reulke. "Investigation of Minority-Carrier Diffusion Lengths by Means of the Scanning Electron Microprobe (SEM)". In: Phys. Status Solidi A 66 (1981), p. 283. DOI: 10.1002/pssa.2210660135.

[142] C Donolato. "Evaluation of diffusion lengths and surface recombination velocities from electron beam induced current scans". In: Applied Physics Letters 43.1 (1983), pp. 120-122. DOI: 10.1063/1.94139.

[143] F Berz and H K Kuiken. "Theory of life time measurements with the scanning electron microscope: Steady state". In: Solid-State Electronics 19.6 (1976), pp. 437445. DOI: 10.1016/0038-1101(76)90003-4.

[144] M S Lundstrom and R J Schuelke. "Modeling semiconductor heterojunctions in equilibrium". In: Solid-State Electronics 25.8 (1982), pp. 683-691. DOI: 10.1016/ 0038-1101(82)90195-2. 
[145] T Malis, S C Cheng, and R F Egerton. "EELS log-ratio technique for specimenthickness measurement in the TEM". In: Microscopy Research and Technique 8.2 (1988), pp. 193-200. DOI: 10.1002/jemt.1060080206.

[146] M Lehmann and H Lichte. "Tutorial on Off-Axis Electron Holography". In: Microscopy and Microanalysis 8.06 (2002), pp. 447-466. DOI:10.1017/s1431927602020147.

[147] H G Wagemann and H Eschrich. Grundlagen der photovoltaischen Energiewandlung. B. G. Teubner, Stuttgart, 1994.

[148] M Uehara, S Mori, C H Chen, and S-W Cheong. "Percolative phase separation underlies colossal magnetoresistance in mixed-valent manganites". In: Nature 399.6736 (1999), pp. 560-563. DOI: $10.1038 / 21142$.

[149] K H Ahn, T Lookman, and A R Bishop. "Strain-induced metal-insulator phase coexistence in perovskite manganites". In: Nature 428.6981 (2004), pp. 401-404. DOI: $10.1038 /$ nature02364

[150] J Hoffmann, P Moschkau, S Mildner, J Norpoth, C Jooss, L Wu, and Y Zhu. "Effects of interaction and disorder on polarons in colossal resistance manganite $\mathrm{Pr}_{0.68} \mathrm{Ca}_{0.32} \mathrm{MnO}_{3}$ thin films". In: Materials Research Express 1.4 (2014), p. 046403. DOI: $10.1088 / 2053-1591 / 1 / 4 / 046403$.

[151] J Norpoth, S Mildner, M Scherff, J Hoffmann, and C Jooss. "In situ TEM analysis of resistive switching in manganite based thin-film heterostructures". In: Nanoscale 6.16 (2014), pp. 9852-9862. DOI: $10.1039 / \mathrm{c} 4 \mathrm{nr} 02020 \mathrm{k}$.

[152] K J O'Shea, D McGrouther, C A Ferguson, M Jungbauer, S Hühn, V Moshnyaga, and D A MacLaren. "Fabrication of high quality plan-view TEM specimens using the focused ion beam". In: Micron 66 (2014), pp. 9-15. DOI: 10.1016/j.micron. 2014.04.011.

[153] Ll Balcells, Ll Abad, H Rojas, and B Martínez. "Material damage induced by nanofabrication processes in manganite thin films". In: Nanotechnology 19.13 (2008), p. 135307. DOI: 10.1088/0957-4484/19/13/135307.

[154] K H L Zhang, G Li, S R Spurgeon, L Wang, P Yan, Z Wang, M Gu, T Varga, M E Bowden, Z Zhu, C Wang, and Y Du. "Creation and Ordering of Oxygen Vacancies at $\mathrm{WO}_{3-\delta}$ and Perovskite Interfaces". In: ACS Applied Materials \&6 Interfaces 10.20 (2018), pp. 17480-17486. DOI: 10.1021/acsami.8b03278.

[155] M Taneya, Y Sugimoto, and K Akita. "Characterization of subsurface damage in GaAs processed by $\mathrm{Ga}^{+}$focused ion-beam-assisted $\mathrm{Cl}_{2}$ etching using photoluminescence". In: J. Appl. Phys. 66.3 (1989), pp. 1375-1381. DOI: 10.1063/1.344439.

[156] N I Kato. "Reducing focused ion beam damage to transmission electron microscopy samples". In: Journal of Electron Microscopy 53.5 (2004), pp. 451-458. DOI: $10.1093 / \mathrm{jmicro} / \mathrm{dfh} 080$.

[157] T Meyer, B Kressdorf, J Lindner, P Peretzki, V Roddatis, C Jooss, and M Seibt. "High-resolution Scanning Transmission EBIC Analysis of Misfit Dislocations at Perovskite pn-Heterojunctions". Submitted to Proceedings of the 19th International Conference on Extended Defects in Semiconductors. 2018. 
[158] T Kramer, D Mierwaldt, M Scherff, M Kanbach, and C Jooss. "Developing an in situ environmental TEM set up for investigations of resistive switching mechanisms in $\mathrm{Pt}_{-} \mathrm{Pr}_{1-x} \mathrm{Ca}_{x} \mathrm{MnO}_{3-\delta}$-Pt sandwich structures". In: Ultramicroscopy 184 (2018), pp. 61-70. DOI: 10.1016/j.ultramic.2017.08.012.

[159] T E Everhart and P H Hoff. "Determination of Kilovolt Electron Energy Dissipation vs Penetration Distance in Solid Materials". In: J. Appl. Phys. 42.13 (1971), pp. 5837-5846. DOI: 10.1063/1.1660019.

[160] H Bethe. "Zur Theorie des Durchgangs schneller Korpuskularstrahlen durch Materie". In: Annalen der Physik 397.3 (1930), pp. 325-400. DOI: 10.1002/andp. 19303970303

[161] J I Goldstein, J L Costley amd G W Lorimer, and S J B Reed. "Quantitative X-ray analysis in the electron microscope". In: Scanning Electron Microscopy 1 (1977), pp. 315-324.

[162] T Brammer and H Stiebig. "Applying analytical and numerical methods for the analysis of microcrystalline silicon solar cells". In: Solar Energy Materials and Solar Cells 90.18-19 (2006), pp. 3021-3030. DOI: 10.1016/j .solmat.2006.06. 010.

[163] A. Rothwarf. "Plasmon theory of electron-hole pair production: efficiency of cathode ray phosphors". In: J. Appl. Phys. 44.2 (1973), pp. 752-756. DOI: 10.1063/ 1.1662257.

[164] S Birner, T Zibold, T Andlauer, T Kubis, M Sabathil, A Trellakis, and P Vogl. "nextnano: General Purpose 3-D Simulations". In: IEEE Transactions on Electron Devices 54.9 (2007), pp. 2137-2142. DOI: 10.1109/ted.2007.902871. 


\section{Danksagung}

An erster Stelle gebührt mein herzlicher Dank Prof. Dr. Michael Seibt für die Ermöglichung dieser Arbeit, seine große Geduld, die unzähligen Diskussionen, Kritiken, Ratschläge und Ermunterungen.

Als nächstes ist Prof. Dr. Christian Jooß zu nennen, für die Übernahme des Korreferats, die hervorragende Kobetreuung, und auch hier viele spannende Diskussionen sowie Kritiken und Ratschläge und die tolle Zusammenarbeit im SFB-Projekt B02.

Der Arbeitsgruppe Seibt in wechselnder Besetzung möchte ich ebenfalls Dank aussprechen für die familiäre Arbeitsatmosphäre, die vielen Mensagänge, gegenseitige Unterstützung, die fruchtbaren Diskussionen und Aktionen auch abseits des Arbeitsalltags. Hervorzuheben sind hierbei für diese Arbeit Philipp Saring, Henning Schuhmann, Arne Ahrens, Sarina Grevsmühl, Pablo Marín Perera, Corin Jung, Tobias Meyer, Hendrik Spende und Tobias Westphal.

Auch dem Rest der IV. Physik gebührt mein Dank für das Leben dies- und jenseits der Physik. Für diese Arbeit hervorzuheben ist die Unterstützung von Jörg Malindretos, Kevin Adner, Rasit Kösker und Thomas Lehmann.

Neben der IV. Physik möchte ich mich auch beim ganzen Institut für Materialphysik bedanken. Benedikt Ifland steht dabei ganz vorne für die Herstellung der Proben und die tolle Zusammenarbeit im SFB-Projekt B02. Auch hervorheben möchte ich die Unterstützung von Jörg Hoffmann, Thilo Kramer, Daniel Mierwaldt, Stephanie Mildner, Emanuel Franke, Timo Wuttke, Birte Kressdorf, Jonas Lindner, Danny Schwarzbach, Vladimir Roddatis, Tobias Schulz, Volker Radisch, Matthias Hahn und Mike Kanbach.

Tore Niermann von der TU Berlin möchte ich für die praktische Einführung in die Holografie und die Durchführung der entsprechenden Experimente danken.

Für die Unterstützung mit dem DENS-Halter gebührt mein Dank Dominik Vogt von LOT und Gin Pivak von DENSsolutions.

Fürs Korrekturlesen dieser Dissertation möchte ich mich auch bei Tobias Meyer, Christian Gobert und Johanna Bachmann herzlich bedanken.

Weiterhin gebührt mein besonderer Dank Sarina Paap, die mir in den letzten zwei Jahren immer den nötigen Anschwung gegeben hat. Auch danke ich meinen Eltern, weiterer Familie und Freunden.

Zuletzt möchte ich mich beim SFB 1073 bedanken, für die Finanzierung, die tolle Arbeitsumgebung und viele tolle Workshops, Konferenzen, Kurse und Aktionen. 


\title{
Lebenslauf
}

\author{
Persönliche Angaben
}

Name: Patrick Peretzki

Familienstand: ledig

Staatsangehörigkeit: deutsch

Geburtsdatum: 10. September 1985

Geburtsort: Fritzlar

\section{Hochschulausbildung}

WiSe 2005/2006 Aufnahme des Studiums der Physik an der

Georg-August-Universität Göttingen

$12 / 2011$

Diplom, Titel der Arbeit:

Untersuchung der Textur von MgO-Schichten in $\mathrm{MgO}-$ CoFeB-Dünnschichtsystemen mittels hochaufösender Transmissionselektronenmikroskopie

seit $02 / 2012$

Promotion unter der Betreuung von Prof. Dr. Michael Seibt an der Georg-August Universität Göttingen 